UC-NRLF

B 4 las bll 


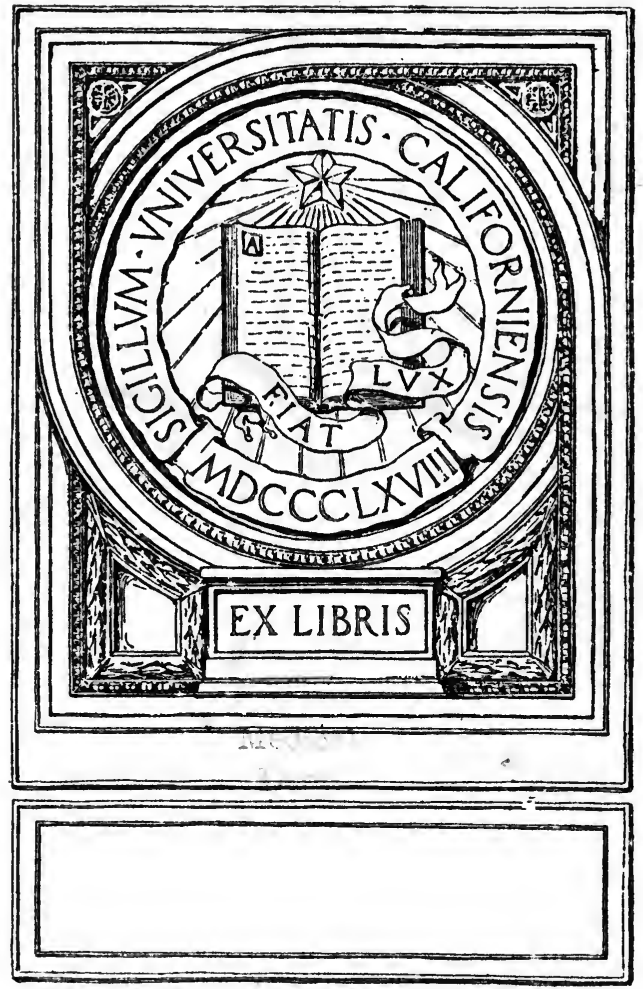



Digitized by the Internet Archive in 2007 with funding from Microsoft Corporation 
THE CONTROL OF HUNGER IN HEALTH AND DISEASE 
THE UNIVERSITY OF CHICAGO PRESS CHICAGO, ILLINOIS

\section{Zqgents}

THE BAKER \& TAYLOR COMPANY NEW YORK

THE CUNNINGHAM, CURTISS \& ẈELCH COMPANY LOS ANGELES

THE CAMBRIDGE UNIVERSITY PRESS

LONDON AND EDINBURGH

THE MARUZEN-KABUSHIKI-KAISHA

TOKYO, OSAKA, KYOTO

THE MISSION BOOK COMPANY

SHANGHAI

KARL W. HIERSEMANN

LEIPZIG 


\title{
THE \\ CONTROL OF HUNGER \\ IN HEALTH \\ AND DISEASE
}

\author{
By \\ ANTON JULIUS CARLSON
}

THE UNIVERSITY OF CHICAGO PRESS

CHICAGO, ILLINOIS 
Copyright igi6 By

The University of Chicago

All Rights Reserved

Published September 1916

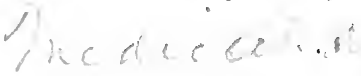

Cumposed and Printed By

The University of Chicago Press

Chicago, Illinois, U.S.A.

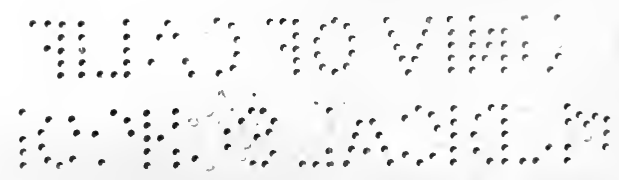




\section{PREFACE}

The following pages contain a summary of the work on the stomach, with special reference to hunger and appetite, carried out in the Hull Physiological Laboratory of the University of Chicago during the last four years. We have aimed to present this digest in the light of the entire biological and clinical literature on the subject, hoping that it may encourage more intensive work on hunger and appetite control, particularly in the fields of clinical medicine and comparative physiology, as the work of the past on this problem is not commensurate with its biological, medical, and economic importance.

The complete analysis of hunger may not yield us control over the hunger mechanism, but it is at least the most promising line of attack. The scientist will concede its value to biology, and the physician readily appreciates its significance for rational therapeutics; but the layman may question the practical utility of hunger control to society as a whole, in view of the fact that it has played no rôle in past evolution. This is granted. But the elimination of many biological correctives by the artificialities of modern civilization calls for rational guidance of all phases of human behavior, including the desire for food. "In these times of plenty, overfeeding, with its physiological penalties and economic waste, is on the whole more prevalent than undernutrition, because of the barbaric indulgence in the pleasures of the table in the absence of the physical stress of more primitive social condition. And when hunger becomes pathologically exaggerated the physician of today knows no remedy; when it fails in disease, he dispenses the "bitter herb" of tradition-and hopes for the best. Hence, when the reader has followed us through these we hope not too technical pages, we believe that he will agree that there is yet much work to be done on the problem of hunger control-work worth doing, co-operative work of the clinic and the biological laboratory.

University of Chicago

A. J. Carlson

September, I9I6 


\section{TABLE OF CONTENTS}

CHAPTER

PAGE

I. The Biological Significance of Hunger . . . . I I

II. HistoricAL . . . . . . . . I6

III. The Stomach in Hunger . . . . . . . . . 30

IV. The Stomach in Hunger (Continued) . . . . . 56

V. Some Accessory Phenomena in Hunger . . . . . . 84

VI. The Relation of Hunger to Appetite . . . . . 96

VII. The Sensibility of the Gastric Mucosa . . . . . io i

VIII. Hunger And Age . . . . . . . . . . . . . . . II

IX. Hunger in Prolonged Starvation . . . . . . 125

X. The Nervous Control of the Hunger Mechanism • . 149

XI. The Nervous Control of the Hunger Mechanism (Continued). . . . . . . . . . . . . $\mathrm{I} 6 \mathrm{I}$

XII. The Nervous Control of the Hunger Mechanism (Continued). . . . . . . . . . . . . . I99

XIII. The Chemical Control of the Hunger Mechanism . . 2 I 7

XIV. Secretion of Appetite Gastric Juice in Man . . . 232

XV. The Chemistry of Human Appetite Gastric Juice • . 248

XVI. Hunger AND Appetite in Disease . . . . . . 26 I

XVII. Hunger and Appetite In Disease (Continued) . $\quad$. $\quad 289$

BIBLIOGRAPHY . . . . . . . . . . . . . 303

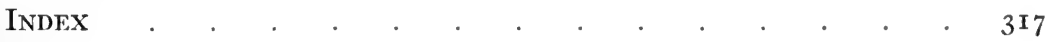





\section{CHAPTER I}

\section{THE BIOLOGICAL SIGNIFICANCE OF HUNGER}

\section{HUNGER IN THE UNICELLULAR ANIMALS}

The complex of sensation that in man and the higher animals urges and compels to ingestion of food is called hunger and appetite. From the standpoint of the persistence of living organisms the ingestion of food is as important as reproduction. Consequently the hunger sensation is as fundamental as the urge or appetite of sex. In fact, if we define hunger biologically as the conditions (rather than the sensation complex) that lead to taking food, hunger is even more fundamental or primitive than the sexual urge (libido), since feeding is a necessity in all forms of life, while sexual reproduction is not.

Whatever be the underlying mechanisms in the genesis of the hunger urge, in the higher animals this urge is obviously a sensation involving a more or less complex nervous organization. Hunger as a sensation or conscious process is therefore probably confined to animals having a nervous system and an alimentary canal. But all living organisms feed. What, then, are the factors that lead to the ingestion of food in unicellular animals and in the simpler metazoa having no specialized nervous system? And are there any essential connections between these primordial factors that cause the ameba to pursue and engulf another moving protozoan, and the mechanism of the hunger urge compelling a starving wolf to chase, capture, and devour a rabbit?

All the unicellular animals live, at least during their periods of activity, in water, in animal and plant fluids, or within the living cells of other animals. In the case of the simpler organisms that are parasitic the conditions of feeding are essentially those of the tissue cells of the higher animals. That is to say, the food materials are in solution in the medium surrounding the cell or the animal. According to Puitter, this also applies to all lower forms of life 
inhabiting the waters of the earth. It is not known to what extent the organic material in solution in the sea water actually sustains the lower animal life, but it is in all probability a very small, if not entirely a negligible factor (Biedermann, Lipschütz, Kerb, Morgulis). We do know that all metazoa feed on other unicellular animals and plants, whole or in fragments. That is, they take into their bodies as food other solid bodies. As regards the actual processes of ingestion of solids by the protozoa, we have practically identical conditions in the case of the special phagocytic cells in the higher animals, although the latter is probably not a feeding process primarily.

What determines the amount and the avidity of food ingestion in the unicellular animals? During the active stage the feeding appears to be on the whole as continuous as contact with food particles permits. Nutrient and non-nutrient particles are taken up somewhat indiscriminately, although there are many exceptions to this rule. In some cases minute motile organisms may by the force of their own motility penetrate into a unicellular animal only to be digested by the latter, but the taking up of solid particles by protozoa is mainly due to active ameboid movements. Augmentation of ameboid movements, increased rate of contraction of pseudopodia acting as feeders, increased ciliary motion both in free swimming and in sessile forms might be taken as external expressions of states of hunger as these would enhance the securing of food.

Are such expressions of hunger state actually present? Jennings, in his studies of the feeding processes of ameba, is silent on this point, except for $\bar{a}$ few incidental observations that an ameba may remain at rest for a few minutes after having taken up a morsel of food, but since the ameba is on the go again before this food is actually digested, the brief rest period cannot be interpreted as a state of satiety. Verworn thinks that all the phenomena of feeding in the protozoa, including a certain capacity of selection of food, involve automatic motility (chemo- and stereotropism) only, but we are not informed whether the rate of this motility varies with the degree of hunger. In vorticella Hodge and Aikens found 
that the cilia worked uniformly and continuously night and day, in drawing in and assorting food particles: "all efforts to surfeit the tiny animals with food produced no appreciable effect in satisfying their apparent hunger." In the presence of an abundance of food the body cilia of paramecium beat less actively, thus bringing the animal to rest, while the oral cilia continue in activity, drawing the food particles into the mouth (Jennings). Wallengren, on the other hand, found no change in ciliary movements and vacuole contractions, or in the excitability of the paramecium during hunger except that ciliary and vacuole activity is decreased when the organism is near death from starvation.

Schaeffer has described in stentor certain differences in behavior between the states of hunger and satiety. When stentor is gorged with food it remains somewhat contracted, the activity of the membranellae are greatly decreased, the animal is less excitable to external stimuli, and it discriminates more perfectly between food particles and indigestible particles in the water current. The degree of satiety appears to depend on other factors beside the amount of food in the body. It seems clear, then, that a state of depletion or hunger in the stentor leads to increased excitability, increased motility, and increased avidity of food ingestion. It is not unlikely that future investigations will reveal similar differences in most of the protozoa. Parker found that meat or meat extract reverses the stroke of the labial cilia in sea anemones, so that the water current carries the food particles into the esophagus. This appears to be an instance of a considerable degree of specialization. Will meat extract in the sea water induce this reversal after the anemone is gorged with meat or other forms of food?

As regards food substances in solution, we may assume that the rate and quantity of ingestion depend on the diffusion rate of the substance and the permeability of cell surfaces. Dilution of food material within the cell may increase surface permeability, and vice versa. We know so little of the correlation of the internal processes in living cells and unicellular animals that we cannot predict what effect scarcity of nutrient material within the cell has on ameboid and ciliary movements, except that when the 
depletion approaches exhaustion these movements probably suffer depression. In some animals partial or complete starvation appears to accelerate metamorphosis and redifferentiation, but the factors involved in these processes are obviously more complex than that of simple and direct cell stimulation.

In the ingestion of organic particles or cells by the special phagocytes in the higher animals, certain new factors enter into play. In the first place, phagocytosis in the metazoa is not primarily a feeding process, but concerned with destruction of cellular débris and cells foreign to the organism. So far as we know, the metazoan phagocytes feed on the organic substances in solution in the body fluids, just like the cells of other tissues. Secondly, the rate and quantity of ingestion of the foreign cells depend in part, not on the condition of the phagocyte, but on certain substances (opsonins) in the body fluids that act on the foreign cells. This factor is not known to be involved in the feeding phagocytosis of the protozoa. Thirdly, even apart from the opsonin factor, certain of the phagocytic cells of the metazoa appear capable of being "trained" to increased activity. The mechanics of this capacity is a matter of conjecture. These differences in the biological meaning of phagocytosis in the metazoa, and the feeding phagocytosis in the protozoa do not imply that the essential mechanics of the phagocytic processes in the two groups are different.

There are-indications, then, that relative depletion of ingested food material, at least in some of the protozoa, results in increased motility (ameboid, ciliary) and increased avidity for food or rate of food intake. These phenomena probably indicate a condition of increased cell excitability. That is to say, a state of hunger in the protozoa is a state of increased excitability. The question how a state of cell hunger causes primarily a state of increased cell excitability cannot be entered into here. To those who prefer anthropomorphic concepts the pursuit, capture, and selection of food by the protozoa become expressions of conscious states analogous to those in higher animals under like conditions. But, for the present, at any rate, simpler explanations are not only adequate, but more useful. The principles of diffusion and selective permeability or 
absorption suffice to account for ingestion of food in solution. The selection of solid food, so far as this principle is in evidence, is probably a matter of inherited mechanism for differential response to chemical and mechanical stimuli. The ameboid and ciliary motility involved in the hunger state introduces more complex factors. Rhumbler endeavored to analyze the former into purely physical surface tension phenomena. Jennings has shown, at least for the ameba, that the surface-tension theory is untenable. But while we are thus forced back on unknown factors in the organization of the cell, there is no reason for believing that when once analyzed these cell processes are not, individually, quite as definitely physical and chemical phenomena as surface tension. Hamburger has recently shown that lack of oxygen acts as a primary stimulus to phagocytosis. But this is in all probability not the mechanism that induces increased cell motility in state of cell hunger, as there is no evidence that a decrease in the food material in the cell is accompanied by oxygen want.

\section{HUNGER IN PLANTS}

This general biological conception of hunger is probably as applicable to the lowest, or unicellular plants, as to the unicellular animals. But we have been unable to find any data bearing directly on this question. We have extensive studies on the chemotactic and general tropic behavior of the lower plants (Pfeffer, Kniep, Kusano, Shibata, etc.). Kniep ascribes senses of taste and smell to bacteria, and shows that the response of bacteria to certain chemical stimuli depends on the reaction of the culture medium, but he does not seem to have raised the question whether the quantity and quality of the food in the culture medium is also a factor in this response. Nor is the question of the effect of starvation on the behavior of bacteria raised in the recent extensive studies on the metabolism of bacteria by Kendall and his pupils.

The swarm cells of the spore-producing plants appear to feed by phagocytosis, just like the unicellular animals, and it is likely that this feeding phagocytosis in plants is influenced by hunger in the same way as in the lower animals. Lister describes the 
ingestion of microspores and bacteria by the swarm cells of mycitozoa, and records the following feeding behavior:

In one instance, after taking in two stout bacilli, and inclosing them in separate vacuoles, the swarm cell remained quiescent for a length of time. I watched the gradual process of digestion of the bacilli. After remaining (quiescent) under observation for nearly an hour and a half the swarm cell swam off with vigorous lashing movement of the cilium.

With the exception of some of the bacteria, the swarm cells of the sporophytes, the group of "parasitic" plants and, to a certain extent, the so-called "carnivorous" plants, the vegetable organisms feed primarily on the inorganic material in the soil and on the carbon dioxide of the air, and in these feeding processes motility of the plant, apart from growth, plays a minor part. The plant cannot move itself, nor can it move its food, even to the extent that both are possible in sessile animals. We may speak with perfect justification of starvation in plants, but in the case of the higher plants there is no evidence that starvation increases excitability and motility, that is, induces a biological state of hunger, nor would such changes aid the higher plants in securing food.

\section{HUNGER IN THE HIGHER ANIMALS}

As experienced by man, the hunger urge is a more or less uncomfortable feeling of tension or pressure and pain referred to the region of the stomach. In normal persons the hunger must become exceptionally strong to be markedly painful. Ordinarily the feeling is one of somewhat uncomfortable tension, accompanied by a feeling of "emptiness" in the epigastric region.

Another characteristic element in the hunger feeling as known in man is its periodicity or intermittency, even when the stomach continues empty and the physical activity of the individual proceeds without interruption. The significance of this attribute has been especially emphasized by Bardier, Sternberg, Polimanti, Cannon and Washburn. It probably applies to the hunger sensation in most mammals and birds. In the lower animals, so far investigated, this periodicity is of a different type, or is possibly lacking. Some persons appear to experience a certain feeling or sensation in the 
esophagus, the throat, and the muscles of mastication synchronously with the gastric hunger. The writer has never experienced these esophagus and throat elements. From all accounts, they differ from the gastric sensation in not being uncomfortable or painful. In the species of birds having a region of the esophagus dilated into a crop, the hunger sensation probably has its origin in the crop rather than in the muscular stomach or gizzard.

The epigastric sensation of varying degrees of pain is, however, the one indispensable element in hunger. But frequently certain accessory phenomena are present. The most common of these is a feeling of general lassitude or weakness. Headache, nausea, nervous irritability, vaso-motor instability, and even fainting may appear as part of the hunger complex. Strictly speaking, a certain degree of nervous hyperexcitability is a necessary effect of hunger of even moderate intensity, and should therefore not be called an accessory phenomenon. But in normal persons with stable nervous organization, strong hunger may be present without any feeling of weakness, headache, or obvious manifestations of nervousness. In some individuals, on the other hand, the feeling of weakness, headache, and general restlessness may be so marked as to crowd out of consciousness the central factor of hunger, the gastric hunger pangs.

In man the genesis of the hunger sensation requires a stomach empty or nearly empty of food. Adult persons eating three to five large meals per day probably seldom experience hunger unless engaged in severe physical labor or exposed to intense cold. In such individuals the nervous impulses from the stomach that give rise to the hunger pangs do not become sufficiently intense to affect consciousness.

We shall show later that the genesis of the hunger pangs is due to certain contractions and tonus states in the stomach, the afferent nervous impulses thus initiated affecting certain parts of the brain. Hunger as known in man thus requires a nervous system, a muscular digestive tract, and an afferent or sensory pathway connecting the two. These anatomical conditions are common to all vertebrates, and to the various invertebrate groups down to and including the coelenterates. 
The influence of the hunger state on the behavior is essentially the same in all these animals. A fundamental characteristic is increased nervous excitability and restlessness. The restlessness is probably not primarily due to the consciousness of the hunger feeling, since it is in evidence in animals deprived of their cerebrum (dogs, birds). The strong hunger urge evidently inhibits fear, as the starving animal becomes more bold and ferocious. It is generally held that the state of hunger in man tends to produce a cantankerous or unsocial disposition. On the other hand, prolonged fasting by the religious devotee is supposed to make him more worthy or fit to commune with the gods.

In the normal man, and probably in the carnivorous animals, an empty or a nearly empty stomach is a requisite for the appearance of the hunger feeling. This is evidently not the case in the ruminating animals and in the herbivora in general, for in these animals the stomach is never empty-not even after days of starvation. Birds, also, feed more or less continuously, even though the crop or the stomach is quite filled with food. Either these animals do feel hunger on a partially filled stomach, or else they eat, not because of feeling the hunger urge, but because of appetite.

On the other hand, hunger may be apparently absent in some animals, even though the stomach is completely empty of food for weeks or months. It is generally accepted as a fact that the Rhine and the Pacific species of salmon do not feed after entering the rivers to spawn, although the fish is doing great muscular work in going hundreds of miles against river currents, in ascending falls, and in fighting rivals. Voit thought that under these conditions the fish cannot be feeling hunger. When in captivity, certain animals may refuse food even to the point of starving to death in the presence of plenty. The call of the empty stomach, if sufficiently strong, wakes up the sleeping child, the sleeping man, or the sleeping dog, but the empty stomach does not appear to disturb the hibernating animal. "The caterpillar," says the great physiologist Haller, "does nothing but eat and defecate." After the caterpillar has turned into a butterfly he may never feed again, particularly if his span of life is a short one, despite great physical 
exertion in flight and reproduction. It does not appear that this diminution or absence of feeding in many insects after the final metamorphosis is associated with atrophy or absence of the alimentary tract. These special conditions obviously involve changes in the brain processes, in the stomach, or in the nervous connections between the brain and the stomach, which must be cleared up by new lines of work; but they do not overthrow the foregoing view of the rôle of the hunger urge in feeding, or the rôle of the empty stomach in the genesis of this urge.

Except in the peculiar and special cases referred to above there is evidence that the intensity or persistence of the hunger urge runs parallel with the degree of activity and the rate of metabolism in the normal individual. Hunger is thus more marked in the young and growing than in the aged and inactive individual. There are indications in mammals that hunger may be experienced even before birth. In warm-blooded animals hunger is augmented by external cold, and depressed by external heat. The reverse is probably true in the cold-blooded animals, but this point has not been experimentally determined. These relations do not obtain in various conditions of disease.

\section{HUNGER, APPETITE, AND THE INGESTION OF FOOD}

Why does this feeling of uncomfortable tension or hunger pang in the stomach induce us to take food? The obvious reply is that we know that eating will abolish the pangs. That is, it is a matter of individual experience. This answer does not seem adequate in the case of the newborn animal. Even if the animal experienced the sensation of hunger before birth, he cannot have experienced what effect the ingestion of food has on this sensation. What induces the newborn animal to take his first meal? Is it a matter of inherited reflexes or "instinct"? Gemelli and others refer to hunger as an "instinct." The animal emerges from the egg or uterus provided with all the essential reflexes, the working order of most of these probably already tried out before entering upon the new mode of living. The feeding reflexes are there; so are the defensive ones in the way of rejection of unpalatable or really injurious substances 
once in the mouth. We have seen that the hunger state of the stomach augments the reflex excitability. This leads to greater degree of moving about on the part of the newborn animal capable of locomotion, and to greater activity of the feeding reflexes in all. This the writer has observed in decerebrated pigeons kept in good condition for months after the operation. When the crop becomes empty this bird not only becomes restless and keeps walking about incessantly, but picks at the floor, the walls of the cage, or the empty air. If it happens to pick at food before it, there is no indication of "recognition" of it as food. Nor does it open its beak in going through the motion of picking. The significant fact in this connection is the inducing of the picking reflex by the hunger state.

In the newborn everything within reach goes into the mouth to be rejected or swallowed according to its chemical character or physical consistency. It would, then, seem that the newborn connects up the gastric hunger urge with the processes of feeding as a matter of individual experience by the method of trial and error. In man there is conscious direction on the part of the mother. Conscious direction as well as the factor of imitation probably plays a rôle in newly hatched chickens "learning" to feed. In the young birds that secure their food by thrusting their beak and head down the throat of the mother, maternal direction is also probably the initial factor. The hunger sensation induces the movements of sucking in the newborn mammal, while in the newly hatched and immature bird it causes the beak to be set wide open. Such minor differences are obviously a matter of inherited reflexes, and we need not fall back on "instincts" to account for them.

On the foregoing hypothesis the connection between the primary sensation of hunger and the processes of feeding is a matter of individual experience by the method of "trial and error." The fundamental factors are the augmentation of all reflexes by the gastric hunger state, the removal of the hunger pangs, and the production of the opposite sensation of satiety by sucking, mastication, and ingestion of food. The theory demands the presence of memory, otherwise each feeding act or feeding period becomes a matter of trial and error. 
We may ask whether this gap between the pure sensation of hunger and the ingestion of food in the newborn is not in reality bridged by the factor of appetite. There is as yet great uncertainty and confusion in regard to the elements of appetite, and the relation of appetite to hunger. Many physiologists appear to accept the view that appetite and hunger involve identical mechanisms and differ only in degree of intensity. That is, a mild state of hunger is called appetite, and a strong appetite is called hunger. There are others who maintain that hunger and appetite are different, both in the quality of the sensation and in the mechanisms involved in the genesis of the sensation. The latter view appears to make the nearest approach to actual conditions.

Appetite, as we know it, cannot be separated from our memory of past experience with food, that is, the taste, smell, and appearance of food. In fact, it appears to be essentially pleasant memory processes of these past experiences, and the "urge" in appetite may be only a special case of the general desire for pleasure. If this is the case, there can be no urge for food in the absence of past experience with food on the part of the individual, and as this is lacking in the newborn, the appetite urge must also be wanting. Hence it is not the factor that guides the newborn individual for the first time to abolish the pangs of hunger by ingestion of food. However, it is conceivable that appetite contains an elemental urge for food as an inherited mechanism and thus not dependent on individual experience, and that when the individual has such experience with food, memory processes of this experience fuse with or overshadow the inheritance factor, so that the two cannot be dissociated in consciousness. This inherited appetite urge, if it exists at all, is probably essentially a positive chemotropism (smell, taste), although birds hatched sufficiently mature to seek their own food probably select or seek the food by vision rather than by smell.

This positive chemotropism for food may in the newborn of the higher animals involve an element of pleasure. It is clear that such an inherited positive chemotropism augmenting the motility and guiding the movements, plus the inherited reflexes of taking 
everything within reach into the mouth, will lead to food ingestion in the newborn, even in the absence of hunger. But if the latter is present the food ingestion completes the experience of removal of the unpleasant hunger pangs by feeding. Loeb and others have referred to a positive chemotropism as a factor in the finding and selection of food, especially in the lower animals, but we do not know whether in the species thus controlled a state of hunger means an increased excitability and augmented motor response to the specific chemical stimuli.

It would thus seem that the first ingestion of food on the part of the newborn or newly hatched animal that feeds unaided by the parents can be accounted for by either of the theories outlined above. Given the hunger pangs with its effects in the way of increased motility and reflex excitability and the inherited reflexes of putting everything within reach into the mouth, plus the reflexes causing rejection of injurious or "disagreeable" material, the ingestion of food becomes only a question of it being within reach, and the experience of removing the uncomfortable pangs of hunger by feeding is quickly established. On the other hand, given an inherited appetite urge or positive chemotropism, so that certain olfactory and gustatory stimuli initiate and direct the reflexes and possibly produce a sensation of pleasure, ingestion of food is equally inevitable.

The first experience of feeding once gained, the individual will feed again because of appetite, that is, the pleasure in the tasting and smelling of food, and the pleasure in the sensation of satiety, or because of hunger and the experience that feeding removes the hunger pains.

In the discussion so far we have used the term "food" mostly in the restricted sense of organic food substances. In a wider sense the term "food" includes all materials necessary for the continuance of the life of the animal. The sensations induced by the lack of water (thirst) and by impairment of the external processes of respiration (dyspnea) have nothing in common with the sensation of hunger (due to lack of pabulum in the stomach), either in their genesis or in their character, except that all three 
sensation complexes are more or less uncomfortable or painful. But we may speak of the sensation of thirst for water as analogous to the sensation of hunger for food. There is, however, no appetite for water analogous to the appetite for food. This is probably due to the absence of taste qualities in pure water, and hence to absence of memory representations of taste. In the case of beer or other artificially flavored drinks that may be taken to satisfy thirst, taste qualities are present, and persons may develop an appetite for these drinks in connection with as well as in the absence of actual thirst, just as we may have appetite for certain foods with or without actual hunger sensation.

\section{V. "SALT HUNGER"}

The condition referred to by physiologists as "salt hunger" is not ordinarily experienced by man, except as a preference for some degree of salt flavor in the diet. But this cannot be the factor that makes some herbivorous animals travel great distances to "salt licks," because the salt is not actually mixed with their food. It is well known that depletion of the sodium chloride content of the blood and the tissues below a certain limit leads to serious disturbances in organ activity, but we do not know their specific effect on consciousness. Possibly it is a feeling of general discomfort and weakness, rather than any specific quality of sensation referred to any one part of the body, although marked reduction of the $\mathrm{NaCl}$ in the blood leads to absence of the hydrochloric acid in the gastric juice and, in consequence, digestive disorders.

The experiments of Forster on dogs and pigeons, and those of Lunin on mice showed that animals succumb sooner on a salt-free diet than if they are given no food at all. The symptoms developed by these animals are: weakness and cachexia, nervous hyperexcitability, tremors, and gastero-intestinal disorders (indigestion, vomiting, refusal of food). It is still an open question whether any animal, man included, actually needs sodium chloride in addition to the salts present in all natural foodstuffs. Smith, the veterinary physiologist, maintains that "both carnivora and herbivora obtain in their natural diet a sufficiency of salts, although there is a 
general impression that the wild herbivora long for sodium. It is quite certain that under conditions of domestication horses can be kept in perfect health without receiving any sodium chloride other than that contained in the food, and the amount of this in vegetable substances is very small." Nevertheless, it is a fact that herbivorous animals, wild as well as domesticated, ingest sodium chloride when available, while this is not done by the out-and-out carnivorous species. History seems to show that it is the agricultural races or tribes that have developed the need or at least the greatest desire for salt. According to Bunge, among the races living exclusively or mainly on animal food, salt is either unknown, unused, or actually disliked, while some of the agricultural tribes in Central Africa cherish salt as ardently as our children desire sweets. It is even believed by some anthropologists that this need or desire for salt or animal food is a factor in cannibalism.

Mungo Park, the traveler, describes his own feelings on longcontinued salt want as follows: "I found the scarcity of salt actually painful. The continued subsistence on nothing but vegetable food produced finally a desire for salt so painful that it can hardly be described." We are not familiar with any prolonged experiments with salt-free diets on man. But persons on ordinary diets who have gone for a long time without sodium chloride complain in general of lack of appetite (owing to the unpalatability of the food), and of some vague or general bodily distress-a condition similar to that induced by monotonous and incomplete diets.

But even if we assume that the herbivora do experience a "salt hunger" in the sense of general bodily discomfort and weakness, or special distress referred to the digestive tract, how do the animals know that eating salt will relieve these sensations, and how do they know where to find the salt? In all probability it is a matter of individual experiences and memory. We may assume that a certain amount of salt flavor is pleasant to these animals as a matter of inherited reflexes or because of its slight stimulating action. Hence they will eat or lick the salt wherever found, irrespectivè of actual need. Now, if actual salt need is expressed in a feeling of general bodily distress, and salt being available, the animal will 
sooner or later encounter the experience that eating salt eases the distress. As long as the calf feeds on the milk of its mother, no "additional salts are required in its diet. But, of course, the calf follows the mother to the "salt licks," and through imitation or curiosity learns the taste of salt as well as the location of the "licks," both before and after he actually experiences salt hunger. Thus by aid of the parents or the herd, the individual experience is established. 


\section{CHAPTER II}

\section{HISTORICAL}

\section{THEORIES OF HUNGER AND APPETITE}

The theories so far advanced to explain the genesis of hunger and appetite fall into three main groups, namely, those involving the stimulation of sensory nerves mainly in the digestive tract- $a$ peripheral origin; those involving a direct stimulation of a hypothetical "hunger center" in the brain by the blood, or by some changes in the metabolism in the center itself-a central origin; and lastly those involving a combination of both central and general peripheral factors of hunger-a general sensation.

Theories of peripheral origin of hunger.-The theories of a purely peripheral origin of hunger may in turn be grouped under two heads, viz., (I) The stimulation of a strictly local group of sensory nerves (mainly in the stomach), and (2) the stimulation of all afferent nerves by some change in the tissues or in the blood. We shall first outline the theories that account for the hunger sensation by the stimulation of sensory nerves in the digestive tract. There are, to the author's knowledge, at least six such theories, namely: (I) Hunger is due to mechanical stimulation of sensory nerves in the gastric mucosa by mechanical rubbing or pressure from contraction of the stomach. . (2) Hunger is due to chemical stimulation of sensory nerves in the gastric mucosa (the acid of the gastric juice, etc.). (3) Hunger is due to the stimulation of sensory nerves in the gastric mucosa by a state of turgescence of the gastric glands. (4) Hunger is due to the stimulation of sensory nerves in the gastric mucosa by some change in the blood due to starvation. (5) Hunger is due to stimulation of sensory nerves in the stomach by the atony and absence of contractions of the empty stomach. (6) Hunger is due to the stimulation of sensory nerves in the wall of the stomach (muscularis or submucosa) especially in the fundus and cardiac regions, by contraction of the empty or partly empty stomach. 
Sternberg's literary essays on hunger and appetite contain numerous references to the conceptions of hunger and appetite held by the ancient and mediaeval poets and philosophers; but there appears to be little or nothing specific concerning the hunger and appetite mechanisms in the writings of either Hippocrates or Galen, although the popular view that prolonged starvation or hunger is extremely painful dates back at least to the time of Homer.

- Hippocrates does remark in one of his famous Aphorisms, that "strong wine cures hunger," but the modus of this cure did not appear to interest him. The theory that the sensation of hunger is due to mechanical stimulation of sensory nerves in the gastric mucosa goes back at least one hundred and fifty years to the great physiologist Haller. On the subject of the immediate cause of hunger, Haller wrote:

Hunger is initiated and intensified by bodily vigor, as in the athlete Milo, in lions, and in all animals possessing great strength by a peculiar strength of the stomach and by physical labor of all kinds, especially at low temperature, since hunger is intensified in cold climates.

Hunger is increased by the presence of intestinal worms, as they consume part of the nutrient juice. It is also augmented by the patency of the pylorus, a condition normally present in voracious animals, and resulting in an almost continuously empty stomach. The sensation of hunger is sometimes even excited by certain acids, such as cream of tartar, lemon juice, and similar substances while regurgitation by putrefying intestinal contents into the stomach depresses and prevents hunger. The numerous kinds of fruits especially enjoyed by the people of the Orient are generally acid.

A greater degree of excitability of the (gastric) nerves also induces greater hunger.

Finally there are imperfectly understood conditions characterized by an inordinate augmentation of hunger, excessive eating, and ingestion of unusual or indigestible substances.

Among animals, those having the shortest span of life, such as the insects, are the most voracious feeders. The caterpillar eats and defecates continuously.

Thus, we see, on the basis of the phenomena of hunger in the entire animal kingdom, the immediate cause of the sensation of hunger is the grinding or rubbing (tritus) of the delicate and vilous folds of the gastric mucosa against each other, through a motion or contraction inherent in the stomach, aided by the diaphragm and the abdominal muscles. These I consider as facts 
already demonstrated. A famous $\operatorname{man}^{x}$ has suggested that the gastric nerves thus irritated are restored by the congestion of blood in the mucus folds, as occurs in all cases of irritation.

We can show, moreover, that the empty stomach is contracted so that no lumen exists. In a hungry man the stomach is contracted (pinched).

From our knowledge of the intolerable sensation produced by rubbing the exposed nerves in a region where the skin has been lacerated or broken, we are permitted to estimate how acute must be the sensation caused by this friction or stimulation of exposed nerves on the gastric mucosa, as in prolonged hunger. I would not care to face such a sinister exitus.

These considerations make it clear why a long-continued fast causes only mild hunger in animals like the snake, in whose stomach there is scarcely any grinding action, as it is not sufficiently muscular; besides the strength of the abdominal muscles and the diaphragm is almost nil. They also explain why chickens die sooner from starvation than do dogs, cats, and carnivorous animals in general. Indeed, the grinding action of the stomach in the gallinaceous birds is stronger than in the quadrupeds. In some insects with feeble peristalsis of the alimentary tract there is no hunger in winter, but in the summer the hunger is greater the greater the heat.

So far Haller. It is probable that Haller confuses hunger with appetite when he speaks of acids or bitter fruits augmenting hunger, although if the sensory nerves of the hunger sense are distributed in the gastric mucosa, the possibility of these nerves being stimulated by certain chemicals in the stomach cavity cannot be excluded. The central facts in Haller's conception are the tonicity and contractions of the empty stomach stimulating the hunger nerves in the mucosa by pressure and rubbing.

Erasmus Darwin thought that the hunger pain is due to the atonicity and absence of contractions in the empty stomach. The view that the empty stomach of normal individuals is atonic and quiescent has persisted in physiological and medical literature to this day, despite both early and recent evidence to the contrary.

Johannes Müller, one of the fathers of modern biology, states that hunger is a kind of negative sensation-or simply due to the absence of the positive sensation generated in the stomach during digestion. If this is the case, an individual should always feel hunger after excision of the stomach or after section of both vagi

${ }^{1}$ The physiologist Senac. 
nerves. Müller notes, however, that the stomach of animals after prolonged fasting or after death from starvation appears very much contracted.

Weber considered it probable that "sudden and strong contraction of the empty stomach, completely obliterating the gastric cavity, gives rise to a part of the sensation we call hunger." As analogies to the hunger pangs he refers to the labor pains (uterine contraction), the pangs from the large intestine in tenesmus, and from the small intestine in cases of colic. Weber does not explain how the gastric contractions stimulate the hunger nerves. This general view that the hunger is caused by contractions of the empty stomach has been accepted by a number of physiologists and clinicians (Vierordt, Hertz, Knapp, and Sternberg), and finally demonstrated by Cannon and Washburn, and Carlson. Voit, Albu, Stiller, Nicolai, and others assume a gastric genesis of hunger without going into the question of how the stimulation is brought about. Sternberg has designated hunger as "Pruritus stomachi," or a tickling sensation, similar to that evoked from mechanical stimulation of certain cutaneous areas. He also suggests that appetite is in some way correlated with the peristalsis of the esophagus and stomach and that absence of appetite or nausea is similarly associated with antiperistalsis. Sternberg's papers on the subject of hunger and appetite contain no original observations, and one meets with a number of contradictions and far-fetched analogies that prove nothing. Thus Sternberg says that "the empty stomach is anatomically an atonic folded tube." Now, an atonic stomach cannot give rise to hunger by contraction or peristalsis, for the simple reason that these are not present, except when there is a certain degree of tonus.

The turgescence theory of hunger as formulated by Beaumont is untenable, for the reason that there is no actual accumulation of gastric juice in the crypts of the glands in the empty stomach to stimulate the gastric nerves by distension. Moreover, there may be a continuous secretion of gastric juice during a hunger period. Yet the theory is accepted, at least in part, by some recent workers (Luciani, Valenti). 
The theory that the sensation of hunger is a mere negative phenomenon, or the absence of the positive sensation accompanying the filled stomach, has not received much attention. If we consult our own experience, the hunger urge appears to be a sensation as positive as pain. We also know that the mere emptiness of the stomach does not initiate the sensation.

The view that hunger is due to the chemical stimulation of nerves in the gastric mucosa appears also to lead back at least to Haller. Soemmering ascribes the hunger pains of fasting to the action of gastric juice on the mucosa nerves. Bostock accepts the theory and credits it to "the chemical physiologists" (the itrachemical school?). Cannon thinks it is based mainly on clinical evidence in cases of so-called gastric hyperacidity and hypersecretion. Pavlov appears to accept it, when he cites his own experience of hunger being initiated by a small quantity of wine passed into the stomach. It is well established, however, that we may feel hunger when the stomach is completely empty of gastric juice and other substances that may be capable of stimulating the nerves in the mucosa. Some mucus is always present in the stomach, but there is no evidence that this can act as chemical stimulus. It is also known that in cases of complete absence of hydrochloric acid in the gastric juice (achylia gastrica) hunger may be present.

Theories of the central origin of hunger.-Magendie thought that hunger is strictly of central origin, not even directly due to depletion of the blood or the tissues, since both the gastric sensation and the general feeling of weakness may pass away without the individual partaking of food. He denied tonic or periodic contractions of the empty stomach, at least during the first 3 to 5 days of fasting, and quotes only to reject mechanical stimulation of the stomach walls, traction on the liver by the diaphragm, fatigue (atony) of the gastric musculature, bile and gastric juices in the stomach as causes of hunger; but he does not deny that sensory impulses for the organs in general may be contributing factors. Tidemann, Schiff, Ewald, Wundt, Milne Edwards, and others adhere in the main to this theory but assume that the hunger center is stimulated by a starvation state of the blood. Many authors have pointed out the 
analogous condition in the case of the respiratory center being stimulated by the venous condition of the blood.

The main objection urged against this theory is its failure to explain, (I) the reference of the hunger sensation to the stomach; (2) the fact that hunger may be temporarily abolished by the eating of indigestible materials, and (3) the periodicity of the hunger sensation.

The main arguments urged by exponents of the central theories against the gastric origin of hunger are: (I) Man and animals will eat after excision of the stomach, or after section of the sensory nerves to the stomach. (2) Hunger may be present even when the stomach is partly filled with food. (3) Hunger may be appeased by feeding per rectum as well as by intravenous injections of foodstuffs.

Hunger a general sensation.-As formulated by Bardier, this theory assumes that the hunger center in the brain is stimulated directly by some change in the blood, and indirectly by afferent nervous impulses from all organs of the body, the stimulation of these afferent nerves being also due to some change in the blood induced by the state of hunger. With certain minor modifications and additions, this view is accepted by Longet, Beaunis, Roux, M. Foster, J. L. Müller, Schlessinger, and others. According to Roux the gastric moiety of the peripheral part of the hunger sense constitutes appetite, that is, appetite is due to the stimulation of sensory nerves in the stomach by the starvation changes in the blood. Müller, writing in I9I5, cannot very well deny the gastric hunger contractions, but he assumes that these contractions are caused through motor impulses in the vagi nerves by stimulation of the hunger center in the brain by the blood, although this possibility was disproved in I9r3 by Carlson. That the stimulation, central and peripheral, is brought about by some changes of the blood in starvation, is the main element in the theory. The analogy of this mechanism to that of the genesis of thirst from the osmotic concentration of the blood has been frequently pointed out in support of the theory. DuBois-Reymond (quoted from Nicolai) postulated a vagus hunger (gastric origin) and a tissue hunger (the 
feeling of weakness). Turro advances a "trophic reflex mechanism" of hunger. Lack of nutrient material stimulates nerve-endings in all organs. The impulses pass to a hypothetical trophic center in the basal ganglia of the brain, and these lower centers, in turn, affect consciousness. He intimates that this "trophic hunger mechanism" is so perfectly adjusted by inheritance that the animal on seeing food knows instinctively, as it were, just how much salt, water, fat, starch, and protein to ingest.

The objections raised by various authors against this theory are: (I) Hunger may set in before intestinal absorption is completed, and therefore before there can be any starvation change in the blood. (2) There are not very marked chemical changes in the blood even in prolonged starvation. (3) Hunger is abolished temporarily by the eating of indigestible matter, and by taking food hunger ceases before any of the food is digested and absorbed. (4) Hunger is usually more or less periodic with sudden onsets and endings, while changes in the composition of the blood from starvation are more likely to be gradual and continuous. (5) In very prolonged starvation hunger does not, at least in man, increase in intensity with the supposed depletion of the blood, and in fevers hunger may be absent despite prolonged starvation with increased metabolism.

That the sensation of hunger is not an evidence of the immediate need of food because of starvation changes in the blood was pointed out by Voit in the following way. The people of Ireland, used to voluminous rations of potatoes, complain of starvation and hunger when given even greater food values, but in smaller bulk. The same is true of the bread-eating peasants of Bavaria when put on a diet of meat.

\section{HISTORICAL STEPS IN THE EXPERIMENTAL INVESTIGATION OF THE HUNGER MECHANISM}

The earliest experimental work on the hunger mechanism was directed to the nerves of the stomach, namely, the vagi and the splanchnics. Sedillot, Bernard, Bidder and Schmidt, Budge, Brunner and Hensen, Longet, Schiff, Ewald, Sherrington, Ducceschi, and others observed that animals will continue to exhibit desire for 
food and to eat after section of the vagi, the splanchnic, and even the taste nerves. Some of these men concluded that hunger does not rise from stimulation of sensory nerves in the stomach, on the assumption that an animal will eat from hunger only. Ludwig, Cannon, and others have questioned the validity of this assumption. It is certain that man at any rate may eat from appetite alone in the absence of hunger, as in the case of eating sweets or dessert at the conclusion of a dinner. Man may even eat from habit or a sense of duty, in the absence both of hunger and appetite, but it is not likely that this applies in a like measure to animals below man. Assuming, for the present, that appetite is in part a memory process, and hence a central nervous system phenomenon essentially, it is clear that section of the vagi would not affect it; hence the fact that an animal eats after section of the vagi tells us nothing as to presence or absence of hunger. Luciani states that fasting dogs refuse to eat for some time after double vagotomy. Valenti cocainized the vagi and the gastric mucosa in fasting dogs and reports that the animals refused food for some hours. These results of Valenti on dogs are called in question by L. R. Müller on the basis of analogous experiments on man. Müller states that cocaine by mouth has little or no influence on the gastric hunger pangs, but it abolishes the feeling of weakness whether the drug is taken by mouth or injected subcutaneously. $\mathrm{He}$ concludes that this is a central action of the cocaine after absorption into the blood.

The advance of modern surgery has made possible the nearly complete removal of the stomach in man and experimental animals. It is reported that such persons continue to experience hunger after the operation, but it is not clear that patients and observers differentiated between hunger and appetite. A dog minus the greater part of the stomach will eat, but we have already pointed out that this is no absolute criterion of hunger. It should be noted here that complete excision of the stomach is impossible, or at least has not yet been accomplished. There always remains a considerable portion of the stomach around the cardia where the union with the duodenum is made, and in healthy individuals at any rate 
this gastric remnant gradually hypertrophies and dilates to form a considerable stomach pouch, sufficient in all likelihood to give rise to hunger pangs, especially as these are primarily originated in the cardiac end of the stomach. The recent observations of Perthes are of importance in this connection. In cases of resection of the stomach for gastric ulcers, Perthes states, when the location of the ulcer is such that the operation removes most of the greater curvature of the stomach body, after recovery $\mathrm{X}$-ray observations show the pylorus permanently open, the diminutive stomach exhibits hypermotility, and the food passes out of the stomach practically at once and without any gastric digestion. All these patients complained of bulimia or excessive hunger pangs. In some cases the hunger pangs were strong enough to wake the persons up from sleep. In order to be comfortable they had to eat every two hours. This excessive hunger did not develop in patients with stomach so resected that the main body or reservoir of the stomach was left intact.

The observations of Busch, in 1862 , on a woman with a duodenal fistula, have been extensively quoted in subsequent discussions on the hunger mechanism. The patient was a woman thirty-one years old. She was greatly emaciated owing to loss of chyme from the fistula, weighing only $68 \mathrm{lbs}$. when she came under Dr. Busch's care. She had inordinate hunger (bulimia) and.felt the hunger even when her stomach was practically filled with food. But taking food into the stomach relieved that part of the hunger which may be described as a painful or gnawing sensation in the stomach. Hunger was also relieved in part by the introduction of chyme into the duodenum. The woman was so emaciated that the stomach and intestinal movements could be seen in detail through the abdominal wall, and Busch observed periods of active peristalsis alternating with periods of rest in the case of the empty intestines. He could not make out any regularity in the recurrence of these activity periods. Busch concludes: The sensation of hunger is made up of two elements. The first is a condition of the central nervous system, in consequence of actual tissue starvation; the second is due to stimulation of nerves in the alimentary tract. 
In extreme starvation the central element persists even when the alimentary tract is filled with food.

Nicolai, in 1892, made some observations on normal persons, and on persons with various gastero-intestinal disorders. He found that the mere act of swallowing or passing the stomach tube abolishes hunger temporarily. Passing water, salt solution, or indigestible material through the tube into the stomach also allays hunger for varying periods. These observations are now readily explained by the inhibitory reflexes from the mouth and the gastric mucosa to the gastric musculature. Nicolai concludes that "the sensation of hunger involves sensory impulses not only from the stomach, but also from the esophagus, and the pharynx," and that appetite and hunger are fundamentally different sensations, either of which may be experienced without the other.

Schlessinger, in 1893, fed normal patients and persons exclusively per rectum for periods varying from I to 20 days. He reports that rectal feeding does not abolish completely the sensation of hunger, even when this feeding leads to an increased body weight. At the most there is a decrease in the hunger sensation for a short time after each rectal feeding. On the basis of more recent work, this is probably due to inhibitory reflexes from the rectum and large intestine to the gastric musculature. Schlessinger reports further that local anesthesia of the gastric mucosa (pieces of ice, chloroform, cocaine in the stomach) causes only a slight or temporary abolition of hunger. But if the persons had been previously fed per rectum, local anesthesia of the gastric mucosa led to complete disappearance of hunger. He therefore concludes that hunger has a double origin: (I) peripheral or gastric, and (2) central stimulation of a hunger center by starvation blood.

Boring, a psychologist, has recently reported a subjective analysis of the character of the hunger sensation, based on the experiences of a great number of persons. He concludes that hunger is a complex of pressure and pain.

Upon a background of dull pressure, which is sometimes recognized definitely as kenisthesis or the equivalent muscular pressure, there is set a dull ache or gnawing pain which characterizes the hunger. Both pain and pressure 
are referred to the region of the stomach. The pain is noted as fluctuating, as rhythmical, as unstable. Three of the observers described in addition a complex kinesthesis in the throat, and oral sensations arising from the free flow of saliva, a complex which meant for them a desire for food or appetite. Here we have the true sensory basis for appetite.

According to Boring hunger is a twofold experience. It is pressure in its weak form, pain and pressure when intense. This recognition of a pressure or kinesthetic element in the hunger pains is important, and has been made by previous observers.

As the reader will recall, the great physiologist, Haller, stated that the "stomach of a starving man is contracted," and the sensation of hunger is due to the rubbing of the folded mucosa by this contraction. But during the one hundred and fifty years since Haller's Elementa appeared the contracted condition of the stomach in starvation has been questioned, denied, or forgotten. Schiff wrote (in 1867): "The movements of the empty stomach are rare, and much less energetic than during digestion," and as late as the year igro Valenti stated that contractions in the empty stomach are rare and feeble. The authors who followed Haller and Weber in accepting the gastric contraction theory of hunger did so essentially without experimental evidence. We take it that all men have observed or experienced rumbling noises (borborygmi) in the abdomen during the period when they feel strong hunger. The noises are due to movements of gas by contraction of some part of the alimentary tract below the esophagus. When the noises' are accompanied by the expulsion of gas or air from the stomach into the esophagus the contractions are evidently in the stomach itself, since this occurs in the absence of contractions of the abdominal muscles and the diaphragm. However, in certain "nervous" persons the borborygmi may be very pronounced without being associated with hunger, and it is obvious that these noises may be produced in the large as well as the small intestines. Hertz thinks that these noises arise exclusively from contractions of the small intestines. Nevertheless, the fact that the borborygmi are so frequently associated with hunger does not appear to have been fully appreciated in connection with the 
genesis of the hunger sensation, until Hertz and Cannon recently called attention to it.

The view that the empty stomach is atonic and quiescent is the more readily accepted, as contractions would seem to have no useful purpose, except when there is food in the stomach. Now and then later observers (Bettmann, Wolff, His) did record that the stomachs of starving men and other animals are tonically contracted, but the first important study and conclusive demonstration of the motor phenomena of the stomach in starvation we owe to Boldyreff in 1905 , although this investigator did not connect the gastric contractions with the genesis of the hunger sensation. Working on dogs with inflated balloons in the stomach, Boldyreff found that the stomach of starving dogs exhibits alternate periods of strong contractions and absolute quiescence, at least during the first 3 to 4 days of starvation. The contraction periods last 20 to 30 minutes, the quiescent periods for $1 \frac{1}{2}$ to $2 \frac{1}{2}$ hours. The period of activity is made up of to to 20 contractions separated by intervals of I to $\mathrm{I} \frac{1}{2}$ minutes, beginning with feeble contractions and gradually reaching their maximum strength at the end of the period. During these periods of gastric motor activity there were also contractions in the intestines. Boldyreff states that the contractions of the empty stomach are stronger than the gastric peristalsis during digestion. There were no contractions in the empty stomach during the periods when the gland secreted gastric juice copiously. Hertz (I9II) gave a different interpretation to these contractions, namely, that they give rise to the hunger sensation.

These are fundamental and important facts. Boldyreff did not think that these gastric contractions gave rise to the sensation of hunger, mainly because they diminished in strength with the length of starvation. He suggests the possibility that the state of hunger in the brain initiates the gastric and intestinal contractions via the motor nerve fibers in the vagi. It seems hardly necessary to point out that the genetic relations of the contractions of the empty stomach to the hunger sensations cannot, be established with certainty on experimental animals below man, because of the difficulty in determining the kind of sensation experienced by the animal: 
In I9ro Hudek and Stigler reported that the stomach empties faster when the food is eaten with hunger than when eaten without hunger. Cannon cites this fact as evidence that the stomach is in greater tonus in the hunger state. It is obvious, however, that an equally important factor is the greater rate of digestion owing to the appetite gastric juice. The difference in the time of emptying of the stomach observed by Hudek and Stigler may be due to this factor alone.

In I9I I Cannon and Washburn by experiments on man (Washburn) proved that the periods of contractions in the empty stomach are synchronous with the periods of hunger sensation, and that each separate contraction is synchronous with a hunger pang. In their experiments a small balloon was swallowed into the stomach and the stomach contractions were recorded graphically parallel with a signal showing when the subject felt the hunger. These observers also obtained evidence of contractions in the lower third of the esophagus, synchronous with the gastric contractions, and conclude that the esophagus plays a part in the genesis of hunger. While noting that the hunger sensation tends to lag behind the gastric contraction both at its beginning and its cessation, their experiments, nevertheless, do not prove that the gastric and esophagus contractions cause the sensation of hunger by stimulation of sensory nerves, but they assume this to be the mechanism. Nor do they indicate, on this assumption, whether the nerves stimulated are those in the gastric mucosa, or in the muscular wall of the stomach. This demonstration of the synchrony of the gastric contractions and the subjective feeling of hunger is an important step, but it does not inform us which is the cause and which the effect. In other words, adherents of central or hunger-center theory may still maintain that the gastric contractions are initiated by motor discharges via the vagi nerves under the influence of a periodic activity in the hunger center in the brain.

A year later (I912) Carlson and his pupils, using essentially the methods of Morat, Boldyreff, Cannon, and Washburn, demonstrated on man and experimental animals that a certain type of contractions in the empty or nearly empty stomach gives rise to 
the sensation of hunger by stimulation of sensory nerves, not in the gastric mucosa, but in the submucosa or muscularis. These hunger contractions of the empty stomach are primarily initiated in the stomach itself and are thus independent of motor impulses from the brain or the spinal cord. The peripheral or gastric origin of the essential element in the sensation of hunger was thus finally established. 


\section{CHAPTER III}

\section{THE STOMACH IN HUNGER}

\section{METHODS OF INVESTIGATION}

Several methods are available for studying the tonus and contractions of the empty stomach both in man and experimental animals, namely: (I) direct inspection by means of a permanent opening (gastric fistula) into the stomach through the abdominal wall; (2) the introduction of a rubber balloon into the stomach, either through the esophagus or through the gastric fistula, and recording the variations in pressure on the distended balloon due to contractions in the stomach; (3) the introduction into the stomach of a balloon coated with bismuth paste on the inside. This permits graphic recording of the stomach movements synchronously with X-ray photography or inspection by aid of the fluoroscope, and, in man, recording the subjective sensations.-

In our work all three methods have been employed. During the last four years the author has been fortunate in having in his service a "second Alexis St. Martin," a man with complete closure of the esophagus and a permanent gastric fistula of twenty years' standing. The gastric fistula is large enough to permit direct inspection of the interior of the stomach, and the introduction of balloons, rubber tubes, and small electric lights for various investigations. This man, Fred Vlcek, a native of Trebone, Bohemia, is now thirty-one years old. For the last twenty years, or since 1897 , he has fed himself through a permanent gastric fistula owing to complete closure of the esophagus, as a result of accidentally drinking a strong solution of caustic soda.

Previous to the swallowing of the caustic the boy had always been healthy. Since the completion of the gastric fistula in 1897 , he has enjoyed good health except that he suffered an attack of pneumonia in 1908. Mr. V. came to America in I9ro. He is in good physical condition, height $5 \mathrm{ft} .8 \mathrm{in}$.; weight, $62 \mathrm{~kg}$. With 
the exception of the closed esophagus and the gastrostomy, the man is in every respect in normal health, and of good average physical development. The size, form, and position of the stomach is that of the "orthotonic" type. The opening into the stomach is on the lesser curvature about $4 \mathrm{~cm}$. on the fundus side of the "transverse band." The fistula is large enough to admit a rubber tube threefourths of an inch in diameter, and a rubber tube of this size is always kept in the fistula. It is usually pushed in to a depth of 3 to 4 inches. The outer end (about 3 inches) is kept corked and bent under the bandage between meals. The tube is changed once a month. Although the tube fits rather snugly in the opening, there is at times some leakage of gastric juice around it, and in consequence some corrosion of the skin for a considerable area around the tube.

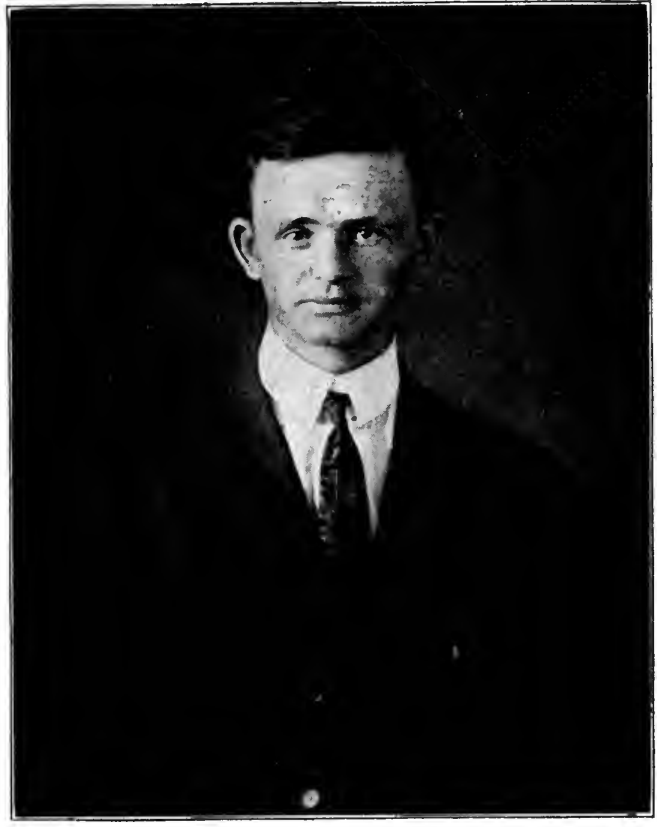

FIG. I.-Mr. F. V. Twenty years after permanent closure of the esophagus and establishment of the gastric fistula.

The stomach mucosa joining the skin or scar tissue has the same appearance and seems to show the same sensitiveness as the rest of the stomach mucosa. X-ray photographs and direct inspection by the fluoroscope show that the esophagus is completely closed at the level of the sternum.

A normal person readily learns to swallow a small rubber balloon with a small flexible rubber-tube attachment, and to keep these in place for hours without the least discomfort or annoyance. There 
is some salivation to begin with, but this soon ceases. Adults as well as infants with this apparatus in the stomach and mouth find no difficulty in going to sleep during an observation period. It is easier to swallow this apparatus than an ordinary stomach tube,

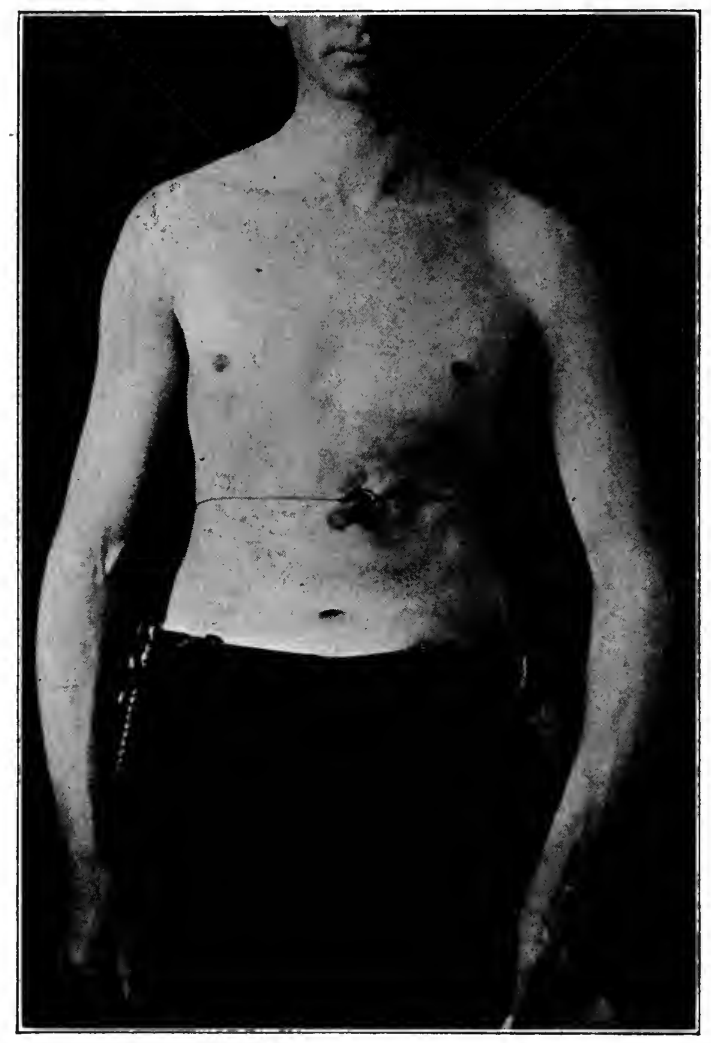

Fig. 2.-Photograph showing permanent gastric fistula of Mr. F. V., with rubber tube in situ.

as the latter is too thick and inflexible. The method has been used on newborn infants with success. It is being used in the author's laboratory by graduate and medical students. The method is so readily mastered as to make it generally available in physiological, psychological, and clinical work. The main points involved 
are: (I) the selection of balloon and rubber tubing of proper size and flexibility; (2) the balloon and tube must be swallowed, not pushed down into the stomach; (3) the avoidance of discomfort or aversion by proper training.

The stomach contractions are recorded from the balloon in the stomach connected by a rubber tube with a water, bromoform, or chloroform manometer. The balloons used by us on man varied in capacity from 75 to $I 50$ c.c. In our observations on infants balloons of smaller size were, of course, employed. And in the work of the stomach (rumen) of goats, balloons of greater strength and capacity are preferable. When bromoform manometers were used a manometer tube $1.5 \mathrm{~cm}$. in diameter was found serviceable. The air pressure in the balloon with the stomach at relative rest was usually adjusted at 3 to $6 \mathrm{~cm}$. of bromoform. After trying out balloons of varying capacities and thickness, we concluded that a condum of the thinnest rubber available in the market was the most serviceable, as this balloon when collapsed is easily swallowed. The advantage of such a delicate balloon is in recording the weaker tonus changes or contractions, and the pulse beat in the stomach. The only disadvantage is the occasional breaking of the balloon by an exceptionally sudden and vigorous stomach contraction, especially when a bromoform manometer is used for recording.

For the convenience of the reader who may not be familiar with the ordinary technique of graphic registration, a diagram illustrating the balloon method as used on man is shown in Fig. 4 in the hope that it may aid in interpreting the various stomach tracings reproduced in this and subsequent chapters.

\section{MOVEMENTS OF THE EMPTY STOMACH IN MAN}

The movements of the empty stomach in mammals were extensively studied by Boldyreff in dogs by means of the gastric fistula. Rubber balloons were introduced into the stomach and connected by air or water transmission to the recording manometer. According to Boldyreff the empty stomach of the dog exhibits alternating periods of complete quiescence during the first three or four days of fasting. The periods of activity vary in length from twenty to 
thirty minutes, and the intervening periods of rest last from $1 \frac{1}{2}$ to $2 \frac{1}{2}$ hours. Both the fundus and the pyloric region of the stomach are involved in the activity of the contraction period, the fundus giving Io to 20 very vigorous contractions. Boldyreff states definitelyand the published tracings seem to support the statement-that

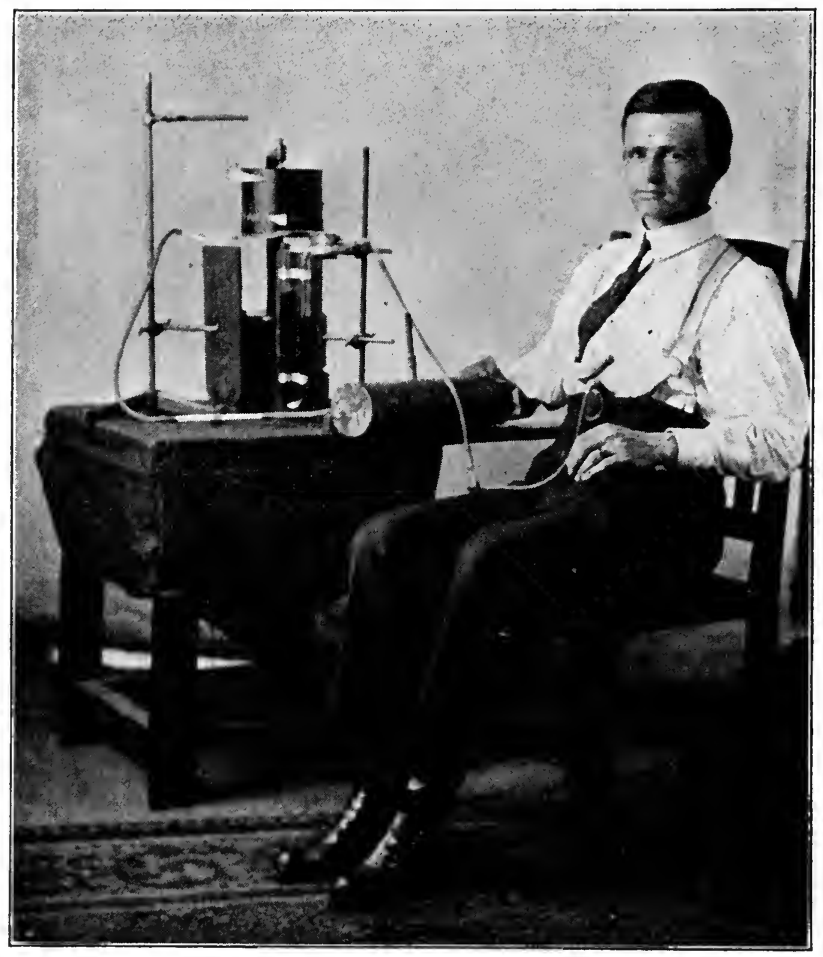

FIG. 3.-Photograph showing arrangements for simultaneous recording of the gastric hunger contractions and the vasomotor and cardiac changes (arm plethysmograph) of Mr. F. V.

between the period of strong rhythmical contractions the stomach is in complete rest. The period of activity begins with weak contractions, and these increase gradually in strength until the period ends abruptly with the strongest contractions. Inasmuch as the tracings published by Boldyreff do not show the stomach 
respiratory pressure or the stomach pulse pressure, it would seem that the methods of registration were not delicate enough to detect feeble rhythms of contractions that might have been present during the periods of relative rest.

Cannon and Washburn studied the movements of the empty stomach in man by introducing a balloon through the esophagus into the stomach. The observations were made 6 to 20 hours after meals. They found that the periodic activity of the empty human

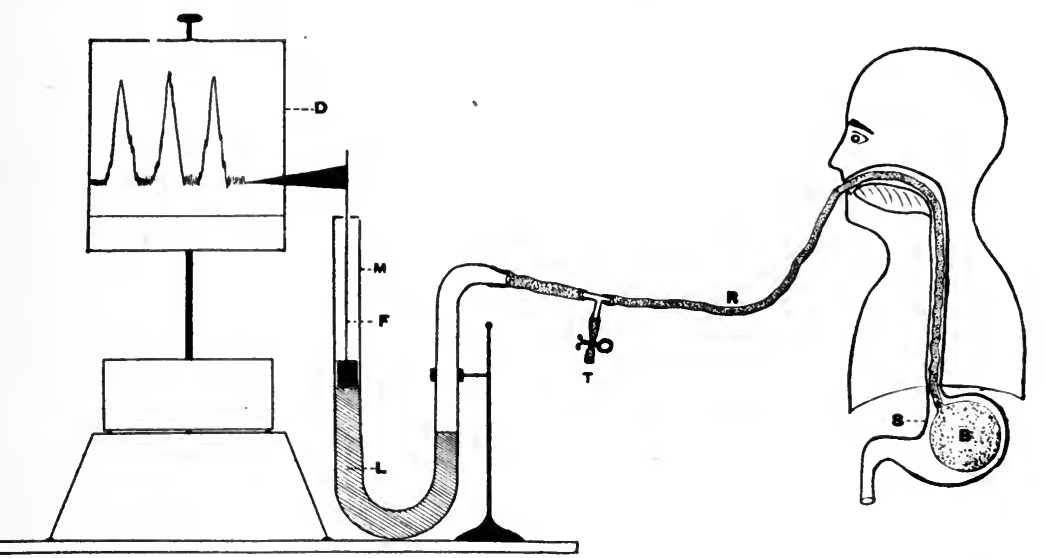

FIG. 4--Diagram showing method of recording gastric hunger contractions of the empty stomach of normal persons. $B$, rubber balloon in stomach. $D$, kymograph. $F$, cork float with recording flag. $M$, manometer. $L$, manometer fluid (bromoform, chloroform, or water). $R$, rubber tube connecting balloon with manometer. $S$, stomach. $T$, side tube for inflation of stomach balloon.

stomach is very similar to that in the dog, but the average duration of the periods is not given. The fundus contractions were about 30 seconds in duration, and the pause between the contractions lasted about 60 seconds. The published tracings show a gradual tonus contraction of the fundus during the pause. The observations of Cannon and Washburn were mainly directed toward establishing the relation between the contraction periods of the stomach and the sensation of hunger. They seem to agree with Boldyreff in the absolute quiescence of the stomach between the periods of strong rhythmical contractions. "Before the hunger 
was experienced by $\mathrm{W}$. the recording apparatus revealed no sign of gastric activity." There is some indication of a feeble rhythm during the rest period in one of their published tracings.

When the pressure in the balloon in the empty stomach of a normal person is properly adjusted and the manometer-recording devices made as delicate as possible, the tracings obtained form a composite of the following pressure variations in the gastric cavity:

I. Periods of powerful rhythmical contractions, alternating with periods of relative quiescence. As the duration of each individual contraction in these periods is approximately 30 seconds, we may call these contractions the "30-seconds rhythm." The entire contraction period we will designate, provisionally, as the "hunger period," and the individual contractions in the period the "hunger contractions."

2. A tonus rhythm (tonus contraction of fundus) of wonderful uniformity in rate, but fluctuating in amplitude, the rate varying from 18 to 22 seconds with an average of 20 seconds. The tonus rhythm increases in amplitude without change in rate during the periods of the powerful rhythmical contractions of the fundus, and are weakest immediately after these periods. But they are always present in the empty stomach of man, provided the subject is in good health. For the sake of brevity we may designate these contractions provisionally as "20-seconds rhythm." The method used by Cannon and Washburn was evidently not delicate enough to detect this tonus rhythm, and hence they concluded, erroneously, that the empty stomach of man is completely quiescent between the periods of the strong hunger contractions.

3. A pulse pressure rhythm, always present.

4. A respiratory pressure rhythm, always present.

The periods of relatively powerful rhythmical contractions (3o-seconds rhythm) are practically identical with the periods of "hunger contraction" of Cannon and Washburn. The individual contractions of these periods usually begin as a feeble tonus rhythm; they gradually increase in amplitude pari passu with shortening of the intervening pauses, and may or may not end in tetanus or 
prolonged tonus contractions, followed by a relatively abrupt relaxation and quiescence.

When the contractions are relatively feeble, the periods of activity are always short, the variation being from 6 to 20 minutes, with an average duration of 12 minutes. The number of strong contractions in these periods varies from ro to 25 , with an average of about I4 contractions. The duration of each individual contraction is approximately 20 to 25 seconds. The stronger contractions are usually in the middle of the periods, the initial and final contractions being the weakest. In no case have we seen such a period end in tetanus.

The periods of more powerful contractions exhibit some characteristic features. The periods are always initiated by weak contractions with long intervening pauses. These pauses may be of several minutes' duration. Then, the individual contractions gradually increase in amplitude, and the intervening pauses become shorter, until the climax is reached in a number of very powerful and rapid contractions approaching incomplete tetanus. The tetanus when present usually lasts from 2 to 5 minutes. The cessation of these periods of activity is always abrupt. In Mr. V. there were at times two or three periods of nearly complete tetanus at the end of the period. On five different days these final tetanus periods lasted for from Io to I 5 minutes. This is, however, exceptional. When the period does end in tetanus, the tetanus usually lasts only 2 to 3 minutes.

This ending of the contraction period in an incomplete tetanus appears to be characteristic of young and vigorous individuals. In older people the period usually ends in a single vigorous contraction without tetanus, except under certain conditions. The ending in tetanus appears to be an evidence of relatively great tonicity of the stomach. In one perfectly normal man (Mr. O.) on whom hundreds of observations were made, the entire period ( 5 to 20 minutes) was virtually a nearly complete gastric tetany.

The duration of each contraction varies from 20 to 30 seconds. The contraction time is shortest at the final stage of greatest activity. When the contrary appears to be the case, the tracings 
show that the prolonged curve is a fusion of two or more contractions. The interval between the contractions varies from 2 to 5 minutes at the beginning of a period to nothing at the end. The duration of the period varies from $\frac{1}{2}$ to $\mathrm{r} \frac{1}{2}$ hours. The usual run is 30 to 45 minutes, the longer periods being exceptional, when there is no experimental interference with the stomach. The number of individual contractions in a period varies from 20 to 70 . The period

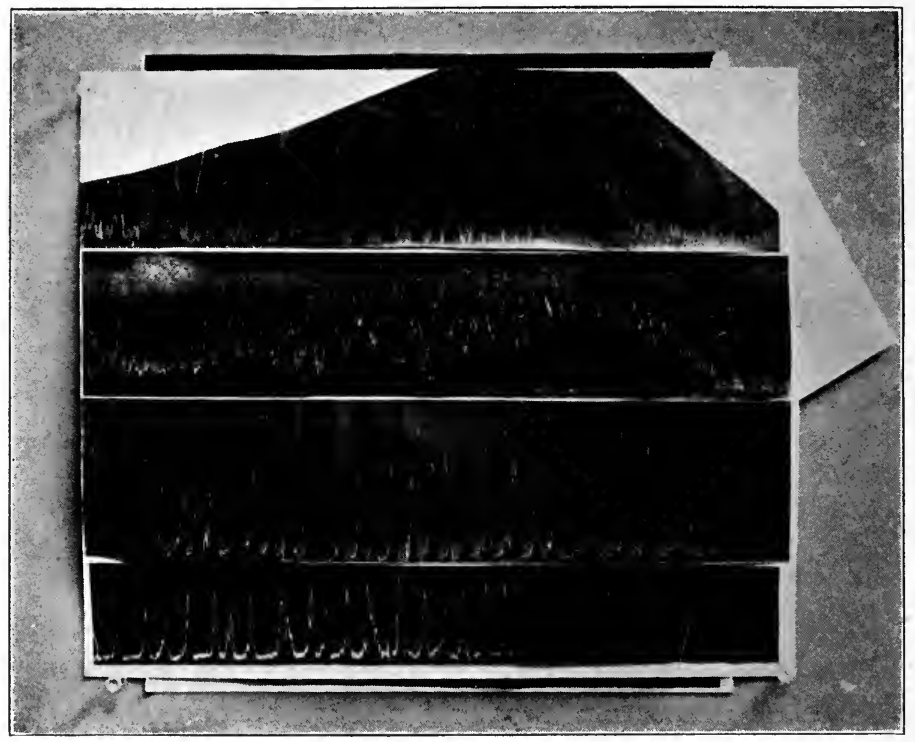

Fig. 5.-The three upper tracings are typical records of the gastric hunger contractions of normal adult persons toward the end of a hunger period. The tracings are recorded and are to be read from left to right, and in each case the gastric hunger contractions cease spontaneously near the right end of the tracings. The more rapid excursions are due to the movements of respiration. The bottom tracing shows typical gastric hunger contractions of a normal dog.

of relative motor quiescence of the empty stomach between the contraction periods varies from $\frac{1}{2}$ to $2 \frac{1}{2}$ hours in normal adult persons. A few characteristic records of these strong periods of motor activity of the empty stomach in normal persons are shown in Fig. 5.

There remains to be noted a rather atypical form of activity of the empty stomach occasionally observed. This consists in 
contractions, feeble or powerful, that do not fall into distinct groups or periods. These contractions are usually irregular both in strength and in rate. The average rate is slow, the interval between the contractions varying from 5 to ro minutes. Similar solitary contractions may also appear in the interval between two typical periods of rhythm. 'These contractions may come two or three in sequence, typical of the beginning of an activity period, but instead of a gradually increasing activity the stomach relapses into relative quiescence for another ro to 30 minutes.

The reader may question the accuracy of denoting these contractions as motor activities of the empty stomach. In all of these cases the stomach was certainly empty of food. But may not the distended balloon act as food, so far as the food acts mechanically in the way of producing stomach movements? This is, indeed, claimed to be the case by Mangold for the muscle stomach of the buzzard. It is not difficult to prove that certain forms of mechanical stimulation, such as the sudden distension of a rubber balloon in the gastric cavity, may cause brief contractions in the stomach, but it can be shown just as conclusively that the stomach rhythms described above are not caused by the presence of the foreign objects in the stomach.

I. The presence of the distended balloon in the stomach between the contraction periods does not induce these contractions.

2. In Mr. V. the gastric contractions can be observed directly through the large fistula without any balloon in the stomach.

3. The contraction periods come on just as frequently without any balloon in the stomach and produce the same effect on consciousness (hunger).

4. In pigeons the periodic strong contractions of the empty crop can be seen directly through the skin and a balloon in the crop does not alter their frequency or intensity.

The stomach pulse.-When the empty stomach is moderately contracted, direct inspection by the aid of a small electric bulb in the gastric cavity shows distinct oscillations of the rugae synchronous with the arterial pulse. The oscillations of the rugae 
cause similar oscillations of the gastric juice (mixed with mucin), which is always present in the otherwise empty stomach. When the strong contractions (30-seconds rhythm) appear, the pulse oscillations of the rugae seem to disappear, either because of the greater rigidity of the stomach folds, or else owing to the difficulty of distinguishing the pulse oscillations when the rugae are closely packed and slide rapidly over and past one another, as they do when the fundus contracts. The picture revealed by the gastric cavity when the empty stomach is in a period of rhythmic contractions is interesting, but rather bewildering, and we have ceased to wonder how Beaumont could have so completely failed to grasp the character of the stomach movements in digestion, as he relied mainly on such direct inspection of the stomach of Alexis St. Martin.

\section{TONUS AND CONTRACTIONS OF THE EMPTY STOMACH IN THE NEWBORN INFANT}

The gastric hunger mechanism is probably inherited. At any rate, the frequency and duration of the periods of gastric hunger contractions are related to the feeding habits of the individuals or the species only so far as the feeding time and food quantity are factors in the time required for emptying of the stomach, and hence for the appearance of the hunger contractions. On the other hand, the hunger mechanism determines to a certain extent the feeding habit. Animals and children probably eat as soon as the stomach is nearly empty, if food is at hand, and the greater frequency of the gastric hunger periods in the young is probably related to the more continuous feeding on the part of the young animal.

We have made observations on a number of newborn infants, with results showing that the empty stomach at birth and in the prematurely born exhibits the typical periods of tonus and hunger contractions of the adult, the only difference between infant and adult being the greater frequency of these periods in the young. In some of the infants the observations were made before their first nursing. It is thus clear that in the normal individual the 
gastric hunger mechanism is completed, physiologically, and is probably active some time before birth.

The recording of the gastric hunger contractions of the newborn human infant offers no great difficulties, if one uses delicate rubber balloons of I 5 c.c. capacity, attached to a flexible rubber catheter of $2 \mathrm{~mm}$. diameter. Most of the infants swallowed this apparatus without difficulty and went to sleep in our arms during the observation periods. The results were always most satisfactory with the infants asleep, as that eliminated all nervous inhibitory factors, and the disturbances from body movements and from irregularities in respiration. Practically nothing can be done with the balloon method if the infant is at all restless. All of our observations were made on healthy and vigorous infants.

In human infants, periods of gastric tonus and hunger contractions are in evidence shortly after birth and before any food has entered the stomach. These gastric hunger periods exhibit all the peculiarities of the gastric hunger contractions of the adult, except that the periods of motor quiescence of the stomach between the hunger periods are on the whole much shorter (Io to I 5 minutes). When the gastric hunger contractions become very vigorous the sleeping infant may show some restlessness, and may even wake up and cry. If the infant is awake the very vigorous hunger contractions frequently induce crying and restlessness. A tracing showing a typical hunger period in a 9-hour-old infant before first nursing is reproduced in Fig. 6. The reader's attention is called to the fact that in infants the gastric hunger periods usually end in

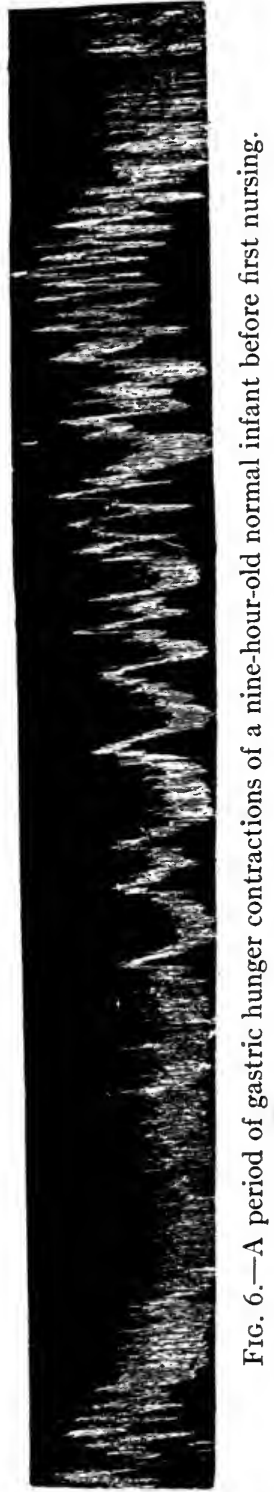
incomplete tetanus, an index of youth and vigorous stomach. 


\section{MOVEMENTS OF THE EMPTY STOMACH IN DOGS}

The contractions of the empty stomach in dogs were recorded by means of a bromoform manometer connected with a delicate rubber balloon in the stomach. In the dogs the balloon was introduced into the stomach either through a gastric fistula or through the esophagus. We were surprised to note the ease with which a small rubber balloon and rubber-tube attachment can be passed through the esophagus into the stomach in dogs. If gentle dogs are selected for the work and the dogs are handled gently, they make little or no resistance after the first two or three experiments. We have never observed vomiting or gagging in dogs as a result of the introduction or the presence of the stomach tube in the esophagus. On the contrary, the dog with the rubber tube and balloon in the stomach and esophagus will lie quietly for hours in the lap of an attendant, while the tonus and movements of the empty stomach are being registered on the kymograph. Frequently the dog will go to sleep during the experiments. This is especially the case if the dog is covered up with a coat or a comforter. The tube in the esophagus does not cause distress or inhibition of the stomach movements. After some training the dogs do not even chew or bite on the rubber tube in the mouth.

Some of the observations were made on dogs with a fistula in the fundus of the stomach. In our first dog we made use of the classical silver cannula. In all the other dogs we discarded the metal cannula and adopted the surgical methods followed in human gastric fistula cases. The incision ( 3 to $4 \mathrm{~cm}$. in length) is made $3 \mathrm{~cm}$. below the last rib and 5 to $6 \mathrm{~cm}$. to the left of the linea alba. The oblique and transverse muscles are carefully separated without cutting them. The desired region of the gastric fundus is pulled out through this opening. The peritoneum is sutured to the fundus pouch. The abdominal muscles are similarly sutured to the pouch. In making these sutures care is taken not to penetrate deeper than the muscle layers of the pouch. The apex of the fundus pouch is then slit open, and the edges sutured to the edges of the skin. A closed rubber tube $\mathrm{I} \mathrm{cm}$. in diameter is passed through the opening into the stomach and kept in place for 4 days. 
Then the tube and dressing are removed. It is found that the abdominal muscles compress this narrow pouch to such an extent that there is virtually no leakage from the stomach, much less leakage, in fact, than even in the most successful fistula using the metal cannula. There is no trouble in closing up of the fistula as long as the animal is being used two or three times a week. The dog takes care of the slight leakage, so there is no corrosion of the skin. We have dogs now in the laboratory with such fistula of I8 months' standing, and the dogs are in the best of condition. In fact, it is obvious that this fistula leaves the stomach much more normal than does the silver cannula method. We have obtained normal hunger contractions of the empty stomach 36 to 48 hours after making the fistula. Nothing like normal hunger contractions is seen in the stomach for 6 to Io days after making the fistula by means of the metal tube. The old silver tube method should be abandoned in all experimental work on the stomach.

In the beginning of this work the animals were kept suspended in comfortable hammocks during the observations on the gastric hunger movements. It soon became apparent, however, that any kind of mechanical restraint on a young, vigorous, and very hungry dog causes restlessness and evident distress, especially when continued for hours. Training will overcome this in part, but not completely. Distress and restlessness will obviously interfere with the stomach movements. We, therefore, tried the expedient of having an attendant keep the dog snugly in his lap during the observation period. This proved very satisfactory, except for the attendant. It is irksome, to say the least, to sit still for 2 to 8 hours at a stretch. We can appreciate the reason for the dog's restlessness when restrained mechanically in a hammock or on a couch for that length of time. When the attendant knows how to handle dogs, even a very hungry dog will lie in his lap quietly for hours, and will usually cuddle up and go to sleep. After a few experiments most dogs seek the research room by preference, and jump into the attendant's lap voluntarily. Some of our dogs became so well trained that they would lie quietly on a pillow for 
2 to 3 hours at a time without any restraint whatever. It is obvious that mental stress and restlessness interfere with the stomach contractions, not only in the way of direct inhibition, but also by the varying tonus and irregular contractions of the abdominal muscles. The animals used in these experiments were mostly young and vigorous females.

The contractions of the empty stomach, as registered by means of a delicate balloon in the fundus, fall into three types according to the degree of tonus of the stomach.

Type I: When the stomach shows feeble tonus, the hunger contractions show an average duration of about 30 seconds, and the intervals between the contractions vary from $\frac{1}{2}$ to 3 or $4 \mathrm{~min}$ utes. This type of contractions usually falls into groups, separated by intervals of relative quiescence. The duration of the groups varies from $\frac{1}{2}$ to 3 hours, and the number of contractions in each group varies correspondingly. It is very rare that a contraction group of type I ends in a tetanus so frequently observed in man. The group usually begins with feeble contractions but of longer than average duration and relatively far apart, and the contractions become gradually stronger and the intervals shorter. The end of the group is usually characterized by contractions of gradually decreasing strength.

Type II: When the stomach is in relatively strong tonus the hunger contractions follow one another in rapid succession, that is, without any intervening pause. The duration of the contractions varies between 20 and 30 seconds. These contractions are frequently interrupted by periods of incomplete tetanus lasting from I to 5 minutes. These periods of tetanus are practially identical with those previously described in man. The contractions of this type do not fall into distinct groups. They may vary to some extent in amplitude and rate, but otherwise be continuous for an observation period lasting from 2 to 6 hours. If the animal becomes restless during the observation period the hunger contractions become irregular and may cease altogether, but this is probably due to splanchnic inhibition, and cannot be regarded as a spontaneous cessation of the hunger contractions. 
This type of hunger contractions seems to be present only in young and vigorous individuals in excellent physical condition. Similar contractions were observed in man, but less frequently than in our young and vigorous dogs. From observations on man, it is certain that the hunger sensation is practically continuous during these contractions.

Type III: The hunger contractions designated as type III constitute virtually an incomplete tetanus of the stomach. This tetanus is characterized by periods of strong and relatively persistent tonus on which are superimposed a series of rapid contractions. The duration of these rapid contractions averages I 2 to 15 seconds. These contractions are evidently analogous to the 20-seconds rhythm in man. These tetanus periods vary in length from I to Io minutes. In prolonged starvation they may last much longer. In moderate hunger they are interspersed between groups of the type II rhythm.

This description of the gastric hunger contractions in dogs is based on observations on more than fifty individuals. The shortest observation period on each animal was two weeks, the longest five months with records taken, on the whole, every third day. The data should therefore be typical. The three types of contractions may be observed in the same dog on different days, or type I may obtain for a few days, and then be superseded by type II, etc. As a general rule type I predominated in some of the dogs and types, II and III in others. Some of the tracings also disclose what may be termed transition stages. Thus, near the end of a contraction period of type I the rapidity of the contraction may approach that of type II, and occasionally the individual contractions of type II will for short periods slow up to such an extent that they parallel type I. This is to be expected, since the types of the hunger contractions seem to vary with the degree of gastric tonus, and this tonus may vary considerably during a single observation period. It is also to be noted that the hunger contractions may occasionally be feeble, irregular, or practically absent for at least 2 to 4 hours at a time in dogs that are seemingly in good condition. And this is usually the case if the dogs are in poor condition from any cause. 
The credit of discovery of the rhythmical contractions of the empty stomach in dogs belongs to Boldyreff, but his account of the rhythm is incomplete and partly misleading. According to Boldyreff the contractions always come in groups of 20 to 30 minutes' duration, and during the $I \frac{1}{2}$ to $2 \frac{1}{2}$ hours' intervals between these groups in which the stomach is completely quiescent. The contractions observed by Boldyreff were evidently short and feeble periods of the type I contractions, but the duration of the interval between the contractions given by Boldyreff is on the whole much greater than that shown in my series. Boldyreff evidently never obtained the rhythm of types II and III in his animals. The difference in the results of Boldyreff and our own are probably due to (I) the condition of the animals, (2) the method of handling the animals, and (3) the method of registering the stomach contractions. Boldyreff used the classical silver cannula for the gastric fistula. This depresses the stomach. All the dogs had in addition to the gastric fistula (fundus) also duodenal, pyloric, pancreatic, or hepatic fistulae. His dogs were therefore subjected to much greater disturbance of digestion and metabolism than is the case of a simple fistula of the fundus as prepared by me. As the dogs were not in the best of condition, it is not surprising that they showed only the feeble rhythm of type I. But it seems likely that forcing the dogs by mechanical means to lie or stand in one position for 6 to I 2 hours at a time is also partly responsible for the brevity of the contraction periods and the length of the intervening periods of quiescence. It is my experience that dogs thus treated become restless, and restlessness always is accompanied by gastric inhibition, probably through the splanchnics. When the dog is allowed to make himself comfortable in the lap of an attendant he lies quietly and usually without any restraint. This condition is certainly more nearly normal.

The tracings published by Boldyreff do not show the respiratory intragastric pressures, nor do they indicate the slightest variations of the gastric tonus during the observation periods. His method of registration was therefore not delicate enough to detect small variations in the intragastric pressure. It would seem, however, 
that his method ought to have recorded the type II contractions, if they had been present in his dogs.

\section{MOVEMENTS OF THE RABBIT'S STOMACH DURING HUNGER}

Rogers, working in the author's laboratory, made gastric fistulas in rabbits by opening the abdominal cavity about I inch to the left of the mid-ventral line and as close to the costal border as possible and suturing the muscularis of the fundic portion of the stomach to the peritoneum and oblique muscles and then the gastric mucosa to the skin. These fistulas were made rather small, so that there would be little leakage of gastric juice or loss of food. - At times a small rubber tube was inserted into the stomach and left there for longer or shorter times to prevent closing of the fistula. Within 36 hours after the operation the animals are usually feeding. These rabbits, if properly taken care of, are in as good condition as normal animals.

Graphic records were made using the rubber balloon method with a manometer pressure of 2 to $4 \mathrm{~cm}$. of chloroform. Mediumsized adult rabbits were used in this set of experiments. In no case did any animal survive a period of continuous starvation of more than 7 days. In animals that died of starvation there were always considerable amounts of residue in the stomach. In the moist condition in which this was removed from the stomach it weighed from 8 to $\mathrm{I} 3 \mathrm{gm}$. Normally the moist contents of the adult rabbit's stomach weigh $90 \mathrm{gm}$. or more. Soon after being deprived of its usual food the rabbit provides a substitute by eating its own excreta. Putting the animal in a wire-bottom cage does not prevent this. The only practicable way to prevent it is to inclose the animal in a cage so fitted with a lid, that the rabbit's head is held outside, its body inside the cage. No doubt such a position proves very irksome to the rabbit, but after becoming accustomed to the position the activities of the stomach were not inhibited. In an animal so fixed, the stomach, as noted by Swirski, empties itself in about 24 hours. Nevertheless, during normal conditions of life the rabbit's stomach . is never empty. 
Since normally the stomach is never empty, it is to be expected that gastric digestion peristalsis will always persist. But as the period of starvation is prolonged, the stomach contractions are altered. Instead of being the gentle peristalsis of normal digestion, they become relatively powerful contraction waves which rapidly follow one another, with a tendency for each peristaltic wave to pass into a short period of tetany. There is no indication of rest or periodicity to these stronger contractions until a short time
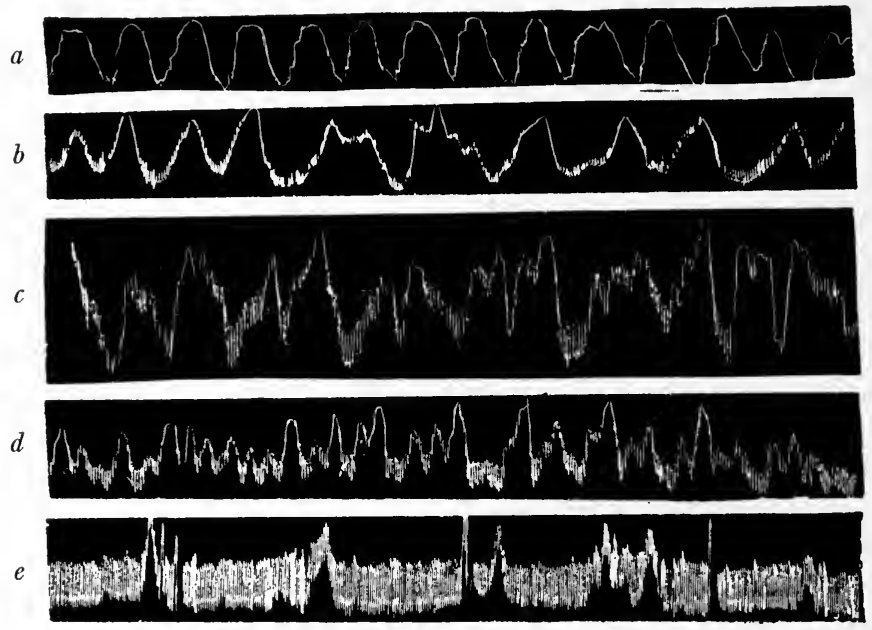

FIG. 7.-Contractions of the rabbit's stomach. $a$, normal digestive peristalsis; $b$, after 24 hours' starvation; $c$, after 75 hours' starvation; $d$, after 92 hours' starvation; $e$, after r ro hours' starvation. The animal died a few hours after this tracing $(e)$ was taken (Rogers).

before death from starvation. Auer has pointed out that during normal peristalsis in the rabbit, the stomach is incessantly active. During hunger the strength of these contractions is accentuated.

Following this period of increased activity during starvation, there comes a period of depression. This is coincident with marked general weakness of the animal or even coma. The decline in the vigor of the stomach activities comes on gradually. The contractions become weaker, of shorter duration, and alternating with short periods of rest. In the last stage of starvation there may 
occur prolonged contractions or periods of tetany lasting from 2 to 3 minutes (Fig. 7).

Gastric tonus variations were frequently seen during hunger, but not commonly during normal digestion peristalsis. May not the increasing muscle tone of the stomach, as starvation is prolonged, play a part in causing the sensation of hunger? Certainly a state of greater contraction of the stomach on the substances remaining within it, as starvation is prolonged, is the first apparent change. Rabbits will show signs of hunger, such as restlessness, gnawing of dry wood, and eating cotton, before the character of the movements as recorded by this method shows any striking change from the normal digestion peristalsis. The increased activity of the stomach that later appears is no doubt also accompanied by psychic changes. The appearance of gastric tetany, if the animal is still able to move about, is marked by restlessness.

In order to determine whether or not the stomach of the rabbit when it contains no food is quiescent, the animal was so caged as to be excluded from its feces. The only difference observed between an animal so caged and one free to move about was that the increased vigor of the stomach contractions comes on sooner in the former. In about I 2 hours after feeding the contractions become very much stronger than normal digestion peristalsis, and this activity both of contractions and tonus variations persists after the stomach has emptied itself of food.

\section{GASTRIC HUNGER CONTRACTIONS OF THE GUINEA-PIG}

Sixteen animals weighing from 450 to $900 \mathrm{gm}$. were studied by Dr. Helene King over periods varying from 13 to 66 days. The guinea-pig is so foreshortened that the gastrostomy operation was beset with some difficulties - the fundic portion of the stomach is pushed up under the diaphragm in such a manner that it must be pulled downward and stitched to the abdominal wall to make a fistula, or an opening must be in the pyloric region. Both methods proved satisfactory, and the possible objection to the lower opening that the balloon did not lie in the fundus was obviated by the use 
of a small balloon or of a finger cot from 3 to 5 c.c. in capacity, pushed well up into the stomach. Several animals killed with the balloon in place left no doubt of the ease with which it was properly inserted. Very small fistulas were made so that when the wounds healed they often measured less than I cm. across. Since the animals began eating within 12 hours, the food in the distended stomach prevented the openings from closing.

The guinea-pig, like other herbivorous animals, feeds at frequent intervals - probably every hour--and under normal conditions the stomach is never found empty. Even within 2 hours after exclusion from food it begins eating its own excreta, a fact noted by other observers, and after I 2 hours will eat paper, pasteboard, or anything of that nature within reach. The easiest and most effective method found for excluding it from its own feces was to place the body of the animal in a bag, sufficiently small to prevent much freedom of movement, and then to draw the bag closely about the neck.

In the guinea-pig contractions of the stomach were observed in 5 hours after taking the food away. Frequently continuous records were made from the time the food was removed until the onset of such vigorous movements. The mild peristaltic waves of digestion become more and more intense until contractions such as might be classified as type I appear-that is, periods of tonus lasting 2 or 3 minutes with 4 or 5 superimposed contractions. This type may continue for 4 hours, but they gradually merge in to the more vigorous type II and possible type III. The contractions follow one another in rapid succession-one in 18 seconds on the averagesuch a period terminating in complete quiescence of the stomach. At times a period of violent coughing precedes the inhibition. Contractions of types I and II have been recorded continuously for 6 hours with but two periods of rest lasting 8 and 6 minutes respectively.

That discomfort is experienced by the guinea-pig when food is withheld for even 4 or 5 hours is evidenced by restlessness, the eating of the animal's own excreta, chewing movements, and sometimes crying, when the contractions are unusually vigorous. The 
animal evidently experiences hunger while the stomach still contains an abundance of food.

\section{CONTRACTIONS OF THE PARTLY EMPTY STOMACH (RUMEN)} OF THE RUMINATING ANIMALS

Like the stomach of the herbivora in general, the various divisions of the stomach in the ruminants are probably never completely empty of food. Digestion in these animals is therefore a more or less continuous process, and if they eat because feeling hunger, they must be capable of experiencing hunger with the stomach partly filled with food, and while the blood is still receiving a constant stream of digested pabulum from the small intestines.

By a balloon method and with a fistula in the rumen, Dr. Schalk and the writer studied the contractions of this stomach pouch in the goat. We started the work on the rumen, as this corresponds to the cardiac or fundus part of the stomach of other mammals, and is, therefore, probably the region most directly concerned with the causation of hunger.

The body of the rumen of the goat exhibits strong periodic contractions, independent of those concerned with the regurgitation of the food bolus into the mouth, i.e., the act of rumination. The contractions vary in intensity, but appear to be practically continuous; that is, there appears to be no period of real quiescence. When the goat is starved for several days or the greater part of the food in the rumen is removed through the fistula, these contractions become stronger without much change in rate. So far, observations have been made only on the rumen of one goat, but the motor conditions found are essentially similar to those already described in the rabbit and the guinea-pig. The digestion contractions of the filled rumen pass gradually into the stronger contractions of the empty or partly empty rumen. We may provisionally call the latter "hunger contractions," assuming that it is the partly empty rumen that gives the impetus to feed. So far as we know the only difference between the completely filled and the partly empty rumen is this difference in tonus and strength of contraction. There is no gastric juice secreted in the rumen, hence there is no 
acidity except that due to the action of bacteria, and there is no regurgitation of the acid content of the true stomach (abomasum) into the rumen. Unless we assume with J. Müller that the sensation of hunger is purely negative, or due to the absence of satiety, the hunger in the ruminant must in some manner be associated with these powerful contractions of the partly empty rumen.

\section{CONTRACTIONS OF THE EMPTY CROP IN BIRDS}

In normal pigeons, with appetites satisfied, the crop is very much distended, and only occasional contractions of the crop can be detected by means of the balloon in the cavity (Rogers). These contractions cannot be detected by inspection of the skin covering the crop. An hour or two after feeding stronger contractions in groups of 3 to 4 appear at 15 - or 20-minute intervals. The motor activity of the crop is gradually increased until, 8 to I 2 hours after feeding, groups of 8 to 20 strong contractions appear at intervals of ro to 30 minutes. Later the crop of young birds passes into a state of continuous rhythmical contractions. When the food content of the crop is reduced to about one-third of the crop's capacity, the contractions are visible through the skin. At this time they involve only the lower part of the crop. When the crop is completely empty the contractions occur in periods of ro to 60 minutes apart, with 8 to 20 contractions in each group. The contractions of the empty crop begin at the upper end, and pass over the entire crop as a peristaltic wave. Each contraction requires I 2 to I5 seconds to pass over the crop. The balloon in the crop does not initiate contractions unless the pressure is excessive. A small fistula in the crop has no effect on the contractions.

It is thus evident that the empty or nearly empty crop of gallinaceous birds exhibits periods of powerful contractions involving the entire organ, while in the filled crop the periodic contractions are confined to the lower end of the organ mainly (Fig. 8, c).

Rogers' studies on the crop in the pigeon have not yet been extended to muscular stomach or gizzard. "According to Rossi the stomach of chickens shows greater motor activity when empty than during digestion, but Mangold states that the empty muscle 

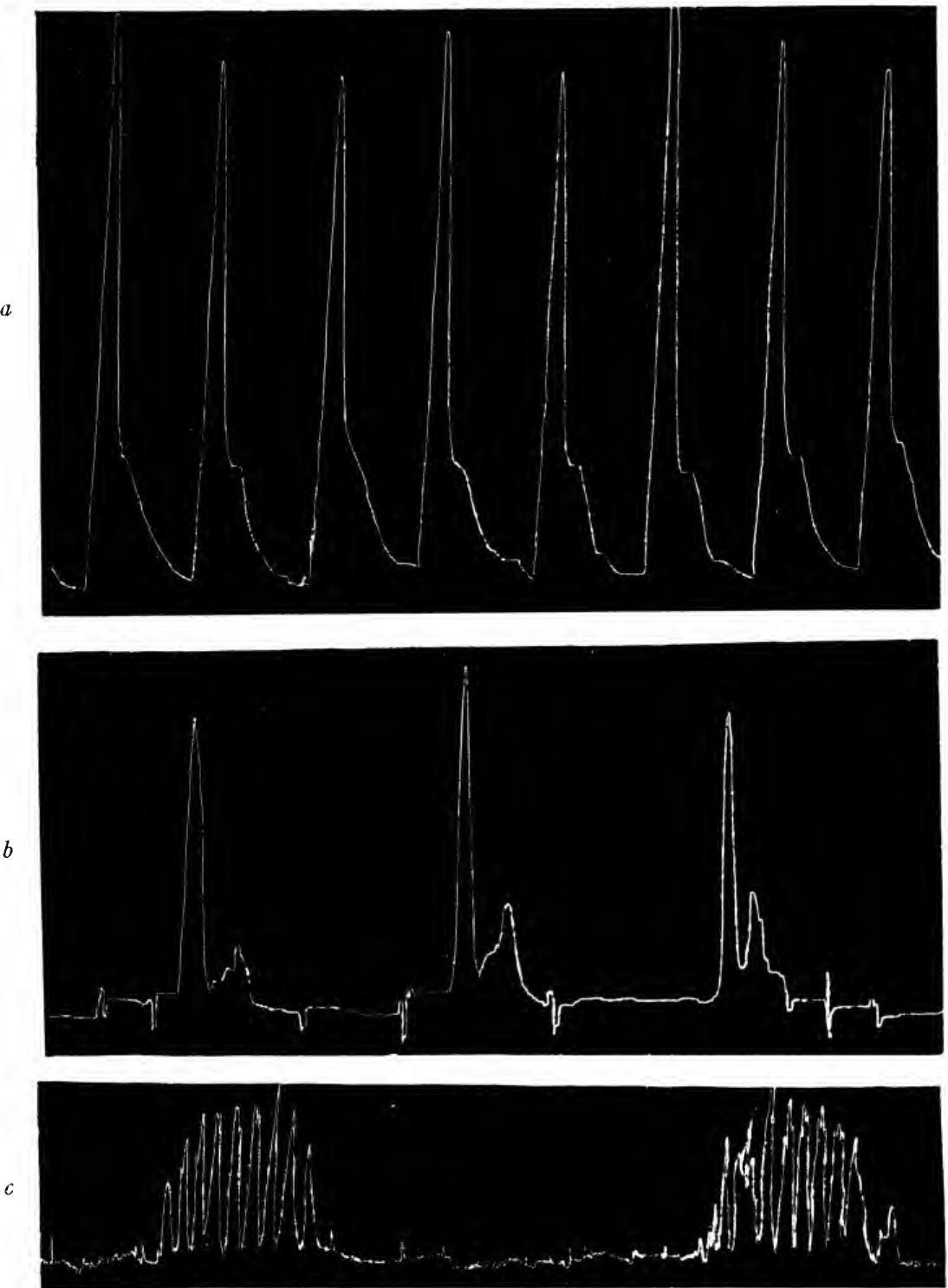

Fic. 8.- $t$, tracings of the gastric hunger contractions of the empty stomach of the bullfrog, I th day of starvation (Patterson); $b$, tracing of the gastric hunger contractions of the turtle, 2 Ist day of starvation (Patterson); $c$, tracing showing the periodic hunger contractions of the crop of birds (pigeon), 2d day of starvation (Rogers). 
stomach of the buzzard is quiescent. Stübel, on the other hand, reports that the empty muscle stomach of pigeons and chickens and ducks shows a rhythmical action current, which obviously implies a corresponding activity or rhythm.

In the species of birds with the food reservoir in the esophagus (crop), the contractions of this region of the esophagus are probably the most important element in the genesis of the hunger sensation, because the crop is emptied sooner than the gizzard.

\section{CONTRACTIONS OF THE EMPTY STOMACH OF AMPHIBIA AND REPTILIA}

Observations (Patterson) were made on the bullfrog (Rana caterbiana) and the common snapping turtle (chelydra serpentina) by the balloon and the balloon X-ray method. In the turtles the balloon was introduced through an ordinary gastrostomy opening. In the frogs a stomatotomy was made by a small incision between the ramus of the inferior maxillary and the anterior coronary of the hyoid bone through the skin, the mylo-hyoid muscle, and the mucous membrane of the throat.

There are slight, if any, tonus variations in the empty stomach of frogs and turtles. In healthy frogs the stomach exhibits continuous rhythmical contractions. These contractions are remarkably strong, sustaining a column I $_{5}$ to $22 \mathrm{~cm}$. high. The contraction phase is abrupt; the relaxation is slower; in fact, it proceeds until the next contraction. The X-ray studies in the intact animal as well as observations on the isolated stomach showed that each contraction is a peristaltic wave starting at or near the cardia and sweeping over the entire stomach, just as is the case with the gastric hunger contraction in the mammals. The striking difference between the bullfrog and the mammal is the absence of periodicity or grouping of the frog's gastric contractions (Fig. 8,a).

The amplitude of these contractions in the frog is increased during prolonged starvation, but there is no obvious increase in tonus. The contractions cease at temperatures above $38^{\circ} . \mathrm{C}$. and below $\mathrm{I} 3^{\circ} \mathrm{C}$. It is significant that the cessation of the hunger contractions of the empty stomach at low temperature $\left(13^{\circ} \mathrm{C}\right.$.) is 
not in tonus, but in atony of the stomach. It would thus seem the gastric hunger mechanism is paralyzed in the hibernating frog. The hunger contractions of the empty stomach of the turtle are very similar to those of the bullfrog. The rate of the contraction is somewhat less, and there are periods of relative quiescence of the empty stomach similar to that in mammals and birds. In prolonged starvation the contractions become stronger and show a tendency to pass into short periods of incomplete tetanus (Fig. 8, b). 


\section{CHAPTER IV}

\section{THE STOMACH IN HUNGER (Continued)}

I. RELATION BETWEEN DIGESTION CONTRACTIONS OF THE FILLED, AND HUNGER CONTRACTIONS OF THE "EMPTY" STOMACH

As we have seen in the case of the rabbit and the guinea-pig and the goat, the digestion contractions of the filled gradually pass into the hunger contractions of the empty or nearly empty stomach. In the frog and the turtle the digestive peristalsis of the filled stomach is practically identical with the hunger peristalsis of the empty stomach. What are the conditions in man and the higher carnivorous mammals? Is it likely that the situation is different in animals with a stomach always more or less filled with food and in animals with a stomach usually empty before they experience hunger?

To answer this question Rogers and Hardt, working in the author's laboratory, used the two standard methods of investigating the movements of the stomach: the rubber balloon and the X-ray methods. The balloon was swallowed shortly after a meal, and continuous graphic records of the intragastric pressure variations were made until after the onset of a typical hunger period. Soon after eating an average meal the subject of the experiment swallowed the balloon and put himself into a comfortable position, either sitting in a chair or lying on a cot. The results were similar, irrespective of the position; the best results were obtained while the subject was asleep.

In order actually to see the hunger movements, we coated a balloon with a bismuth paste and observed its movements in the stomach with the X-ray. These balloons were prepared by painting the outer wall of one with a paste made of bismuth subnitrate and vaseline. This balloon was then inclosed in another of the same size; hence the two balloons were separated by a thin wall of bismuth paste. One is thus able to make graphic tracings with simultaneous direct fluoroscope observation. 
Our records show that the fundus is quiescent immediately after a large meal has been eaten. The pressure upon the balloon is maintained at a steady level. If a light meal is taken, the tonus variations may be demonstrated immediately after eating. At first they are so slight as to seem insignificant, but they increase in vigor and are usually visible 30 minutes after the meal. In one experiment an unusually large meal was eaten and the tonus waves were distinctly in evidence 20 minutes later. They increase in intensity and may, but do not always, become more rapid. Each wave is of $\mathrm{I}$ to 3 minutes' duration. When the stomach is

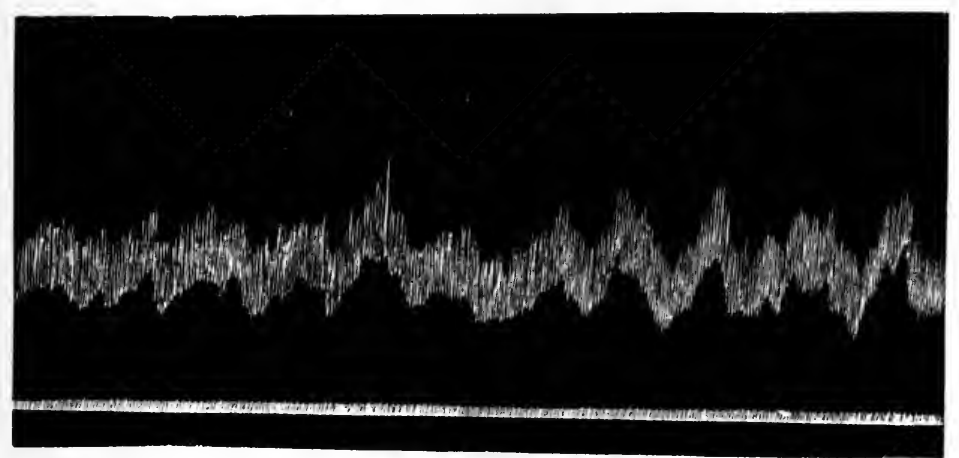

FIG. 9.-Tracing showing the tonus rhythm of the stomach (man) 3 hours after dinner (beefsteak, spaghetti, bread, butter, apples and cream, milk).

nearly empty (as determined by the stomach tube or induced vomiting) they become conspicuous, and at this stage of the digestion there usually appear, superposed upon them, stronger contractions which increase in vigor and are felt by the subject as hunger pains. Although it is by no means always the case, it is significant that the first contractions felt as a hunger pang in man may occur when the stomach still contains traces of food.

We have seen that the onset of a hunger period is marked by the appearance of a slow tonus rhythm which gradually increases in vigor and culminates in the hunger contractions. This tonus rhythm is present, not only as an immediate precursor of the hunger period, but also throughout the course of normal gastric digestion. 
Other workers have employed the rubber balloon in recording the digestion movements in the stomach, but none of the published tracings, so far as we are aware, indicate the presence during normal digestion of the slow continuous rhythm here described. Moritz reported contractions of the fundic end of the stomach occurring $2 \frac{1}{2}$ to $3 \frac{1}{2}$ times per minute. According to Dietlan the

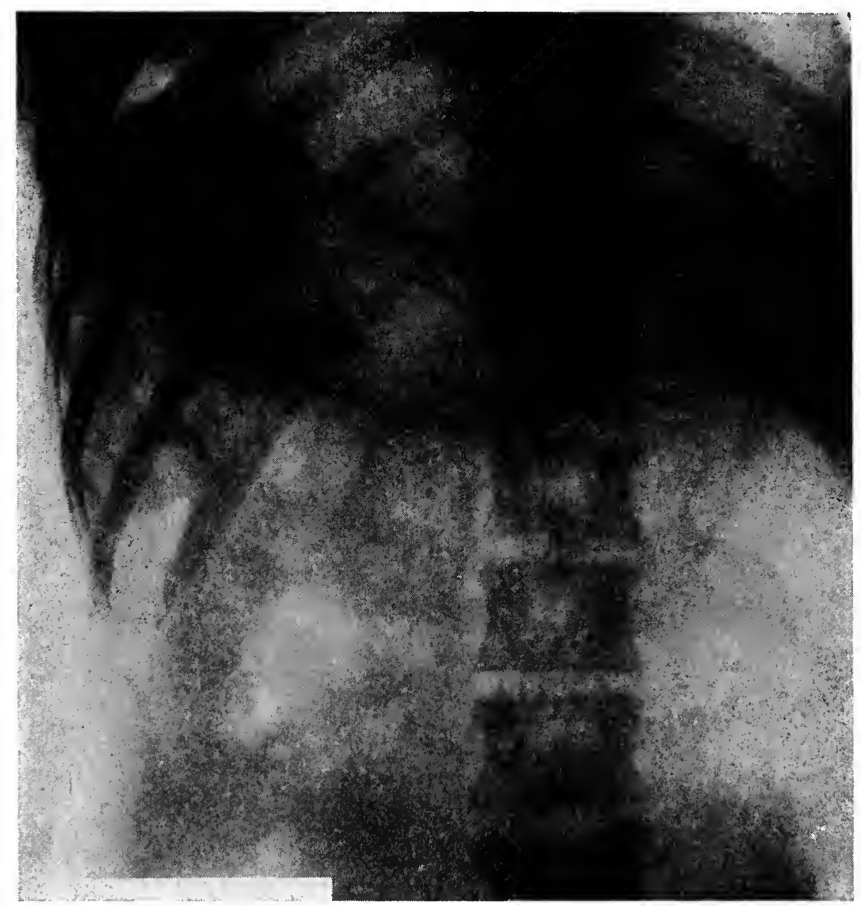

FIG. Io, A.-X-ray photograph of the appearance of the bismuth-coated balloon in the stomach (man) when the empty stomach is quiescent.

time required for the peristaltic contractions of the pyloric end of the stomach is 20 to 24 seconds. The tracings of Moritz probably record the intragastric pressure variations due to the pyloric peristalsis. Sick, using the balloon method, describes tonus variations of the fundic end of the stomach which give rise to the peristaltic waves. These occur at the rate of 2 to 4 per minute in the 
full or empty stomach. The tracings of Sick show three kinds of pressure variations: respiratory, cardiac, and "Magen tonus schwangungen," the latter averaging 20 seconds each in duration. The duration of these "stomach tonus variations" coincides with the time intervals required for the pyloric peristalsis.

Is the hunger contraction simply an augmented peristaltic contraction or a contraction of the fundus as a whole? To answer

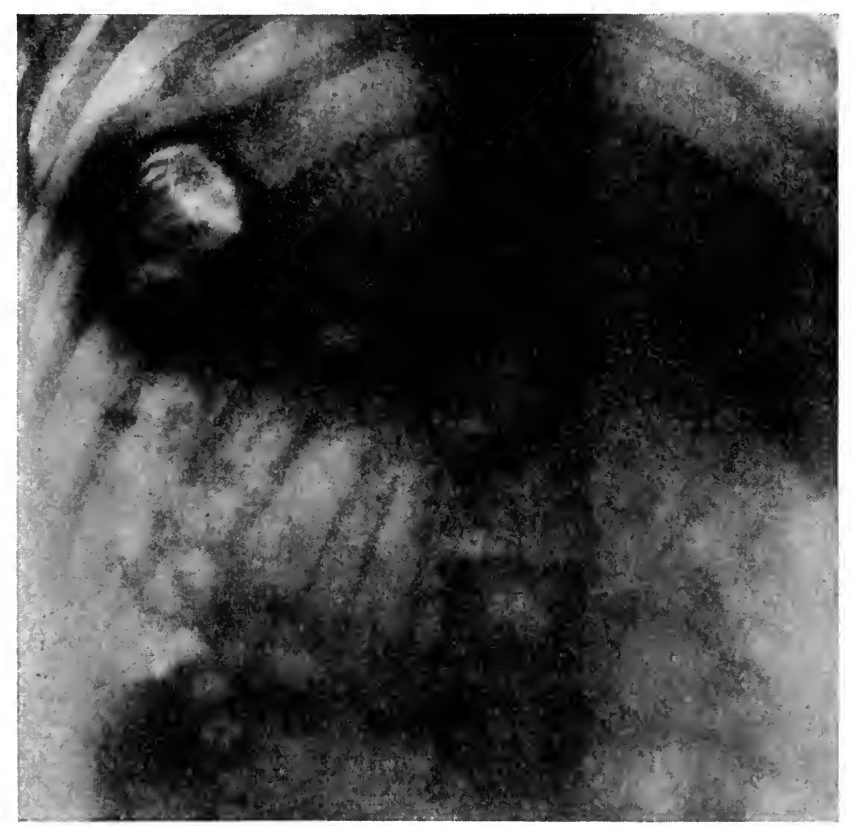

Fig. Io, B.-X-ray photograph of the bismuth-coated balloon in the stomach (man) at the height of a gastric hunger contraction (gastric hunger pang) (Rogers - and Hardt).

this question the stomach movements of the dog and in man were studied by the "balloon-X-ray" method. We were able to make direct observations of the movements of the balloon in the stomach and at the same time to note the character of the graphic record. The upper part of the balloon was held in the cardiac end of the stomach. A young and vigorous dog was employed for the experiments. The dog was starved for intervals of 36 to 48 hours, and 
in order not to have the hunger contractions completely inhibited by the excitement attendant upon the $\mathrm{X}$-ray examination, frequent repetitions were made until the dog became accustomed to the necessary manipulations. The vigor of the stomach contractions thus made visible to the naked eye is surprising. The weaker type of hunger contractions begin as a constriction in the cardiac end of the stomach and pass down toward the pyloric end as a rapid peristaltic wave. In the very vigorous contractions the wave spreads over the stomach so rapidly that it is difficult to decide whether there is. a contraction of the fundus as a whole or a very rapid peristalsis. This contraction may well be compared to the peristaltic rush of the intestine as described by Meltzer and Auer.

The balloon-X-ray observation of the hunger contractions was made on Mr. R. when in a reclining position. When a hunger pang was felt, and the recording manometer showed the typical rise in the intragastric pressure, a series of constrictions were seen passing rapidly over the balloon. Beginning at the cardiac end, they swept rapidly toward the pyloric end, increasing in strength as they proceeded. It was readily seen that the hunger contractions are powerful peristaltic contractions, which, arising at or near the cardiac sphincter, swept downward over the entire stomach. During a typical hunger period the stomach exhibits movements which resemble very closely the movements which have been described by some clinicians in patients after a bismuth meal as hyperperistalsis, but described by Cole as normal peristalsis of a stomach that contains small quantities of food. Observations on the dog were made after 36 hours of deprivation of food, on man after I $_{5}$ hours. Prolonged starvation in the case of the dog was necessary to overcome the inhibitory influence of the excitement attendant upon the X-ray examination. Whether or not the stomach of man after a long period of starvation would show a condition approaching that described for the $\mathrm{dog}$, we are not in a position to state.

The current teaching with reference to the part played by the fundus during digestion is that it is a reservoir, exerting a tonic 
grasp upon its contents. The kinematographic figures of the stomach published by Kästle, Ridder, and Rosenthal show that the fundus is not quiescent during digestion. Cole has shown that when food is in contact with the cardiac end of the stomach in man, contractions begin in the fundus and frequently are as deep

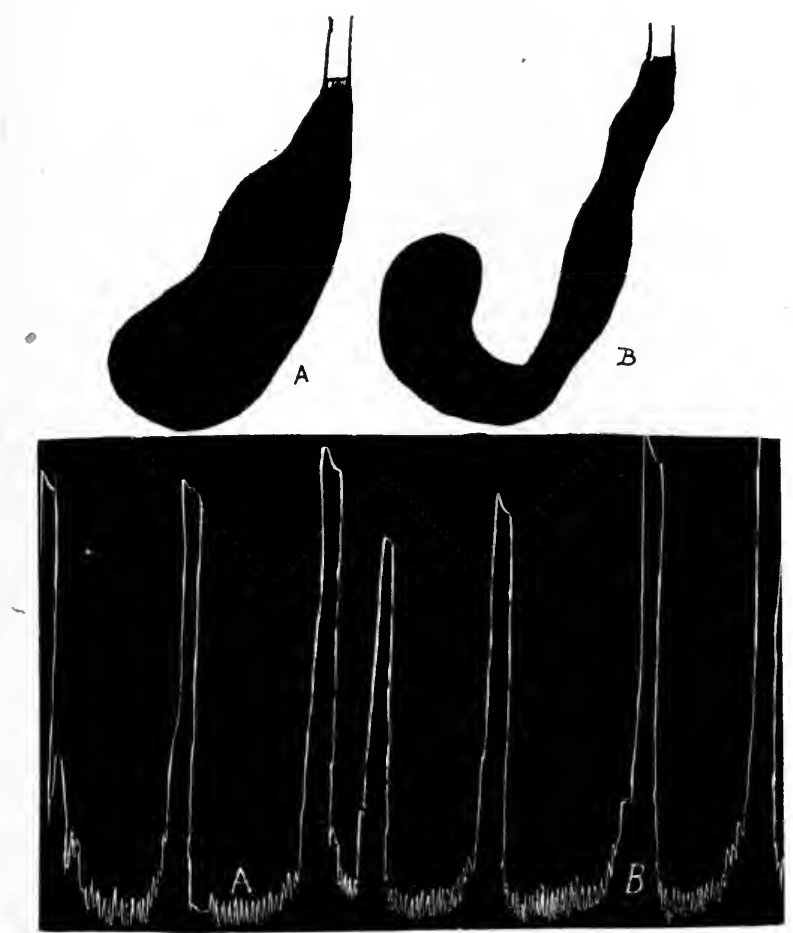

FIG. II.-Hunger contractions of the dog's stomach 30 hours after a meal. $A$, outline of bismuth-coated balloon in stomach between the gastric contractions; $B$, outline of balloon at the height of a hunger contraction (Rogers and Hardt).

in this region as in the pylorus. According to Forsell, with the subject in the standing position, the fundus shows no peristalsis. In the reclining position there occur typical peristalses of the fornix. At the conclusion of gastric digestion there occur circular contractions of the wall. 
It is thus evident that the digestion contractions of the filled stomach pass gradually over into the hunger contractions of the empty stomach. This explains how the hunger sensation may be aroused by the stomach contractions before the stomach is actually empty. It is merely a question of intensity of the fundus contractions. But it is still true that the digestion contractions are primarily concerned with the pyloric end of the stomach, while the hunger contractions of the empty stomach are initiated at the cardia and involve the entire stomach.

\section{ORIGIN OF THE HUNGER SENSATION}

We have now discussed in some detail the tonus and the contractions in the empty or partly empty stomach, esophagus, and intestine of man and of a number of other species of vertebrates. Do these contractions initiate the sensation of hunger, does the central state or sensation of hunger initiate the contractions, or is there no genetic relation between the two series? We have seen that these questions have been answered in various ways by physiologists, psychologists, and clinicians, but without conclusive proofs.

We owe the actual demonstration of the synchrony of the hunger sensations with the strong contractions of the empty stomach to Cannon and Washburn. These observers recorded by means of an electric signal the subjective sense of hunger simultaneously with the intragastric pressure, and found that the stomach contractions and hunger sensations ran parallel. The fact that the beginning of the stomach contractions is in evidence before the hunger sensation is felt and that the sensation lasts longer than the active phase of the contraction is adduced by them in support of the view that contractions in some way stimulate afferent nerves in the stomach, and that these impulses give rise to the hunger pangs.

The beautiful demonstration of Cannon and Washburn leaves undecided the question where the contraction stimuli act in the stomach, and the cause of the peculiar periodicity of these contractions. But there can be no further question of the parallel between the stomach contractions and the hunger sensation, even 
though the evidence that the former are the cause of the latter is incomplete. We do not appreciate the force of Cannon's argument that no other condition than the contractions as the cause can account for the periodicity or intermittency of the hunger sensations. Assuming that the stomach contractions constitute the primary stimuli in the genesis of hunger, does that really solve the problem of periodicity? It would seem that the problem is only shifted a little; for these stomach contractions must depend on corresponding periodic rhythmical activities in the gastric muscles (idio-muscular contractions, "nodal tissue" rhythm) or in central or peripheral motor nervous mechanisms. That such rhythm should give rise to the hunger sensations indirectly through contractions in the digestive tract is just as difficult to explain as a similar central nervous rhythm giving rise to or constituting the hunger sensation directly.

In our experiments the subject was either standing, sitting, or lying down. His position was such that he could not. see the kymograph or any of the recording apparatus. The signal key or keys for recording the hunger sensation were placed in his hand, and he was instructed to press the key as soon as he felt hunger and to keep on pressing it till the hunger was no longer felt. There was no difficulty in keeping a person's attention fixed on this for shorter periods of $\mathrm{I}$ or 2 hours and under conditions of hunger of moderate intensity. But in the case of Mr. V., when the observations were continued without interruption for 5 to 6 hours and, therefore, during several periods of strong hunger, he would usually become restless, and unable to give undivided attention to the introspection.

Most of the observations were made within a period of from 4 to Io hours after meals, and only a few as long as 24 to I 20 hours after a meal, for the reason that the hunger pains in many persons become gradually severe to the point of discomfort, and the man becomes restless and tired.

As a check on the intragastric respiratory pressure, records of the respiratory movements (chest pneumograph) were usually taken simultaneously with that of the stomach movements. 
The general results of our work on more than fifty men are in complete accord with those of Cannon and Washburn. When the empty stomach shows strong contraction, the subject invariably signals that he feels hunger, and, on being questioned, he invariably replies that he feels the hunger in his stomach. There is, on the whole, a fairly close correspondence between the duration of stomach contractions and duration of the subjective sensations of hunger. On days when the stomach does not exhibit these strong contractions, the person does not feel hungry. These "hunger contractions" of the empty stomach are primarily those of the strong periodic rhythm already described.

Relation between the strength of the stomach contractions and the intensity of the hunger sensation.-Data on this point were obtained in the following manner: Three signal magnets were arranged to record on the drum perpendicularly to the recording point of the bromoform manometer, and the corresponding keys were placed in the subject's hand. He was then instructed to press key No. I when he felt, without question, even the faintest hunger; No. 2 when he felt hunger of moderate strength; and No. 3 when he felt the strongest hunger.

The subject presses key No. I (weak hunger) at the beginning of a contraction period when the individual contractions are relatively feeble. Then, as the contraction increases in strength, there comes a period of vacillation between key No. I and key No. 2 (moderate hunger). As the contractions grow still stronger, key No. 2 is used for a while without any change. Then follows a period of alternation between key No. 2 and key No. 3 , and in the final stage of maximum activity of the contraction period the signal is made with key No. 3 exclusively. In other words, there is a fairly close correspondence between the strength of the stomach contractions and the degree of hunger sensations experienced simultaneously.

This account applies particularly to the first hunger period appearing after a meal and for the milder hunger periods in general. On more prolonged fast, that is, after having experienced several hunger periods in succession, the subject may not signal with key 
No. I at all, and sometimes not even with key No. 2, but starts in with key No. 3 (strongest hunger) at the very beginning of a period, despite the fact that the strength of the stomach contractions is not greater (or might be even less) than those designated as very mild or moderate hunger some hours or days earlier. This seems to indicate an increased excitability of the afferent nerves in the stomach, or an increased excitability of some parts of the brain.

The close parallel between the degree of the stomach contractions and the intensity of the hunger sensations is further shown by the fact that the beginning of a strong contraction is frequently signaled by key No. 2 (moderate hunger), and then a shift made to key No. 3 (strong hunger) nearer the apex of the contraction. Evidently, it is possible to distinguish a gradually increasing intensity of the hunger sensation during and parallel with the individual hunger contractions in the stomach. But this distinction is never made in very strong hunger and corresponding contractions.

Fusion of the hunger sensations into hunger tetanus parallel with strong and rapid contractions or tetanus of the stomach contractions.The essential features and conditions of the incomplete tetanus of the stomach contraction at the end of the period of very vigorous contractions are already familiar to the reader. These tetanus periods of the stomach are invariably accompanied by a similar fusion or tetanus of the hunger sensation. The fusion of the hunger sensation appears to be more complete than the fusion of the stomach contractions. When the rate of the strong stomach contractions approaches 2 per minute, the fusion of the hunger sensations is practically complete, that is, a person is then unable to distinguish any rhythmical variations in the hunger intensity. These phases of the stomach contractions are always signaled with key No. 3 (strongest hunger). The greater fusion of the hunger sensations than is shown by the synchronous stomach contractions is probably due to the tonus element, as a strong and persistent state of tonus gives rise to a continuous hunger sensation. The fact that the strong individual sensations lag or persist longer than the corresponding stomach contractions may also be a factor. 
The abrupt cessation of the gastric tetanus at the end of a strong contraction period is accompanied by an equally abrupt and complete cessation of the hunger sensations.

Individual contractions of the "20-seconds rhythm" may give rise to a mild hunger pang, in which case they are usually stronger than those not definitely recognized in consciousness. But occasionally there may be no marked difference in the amplitude of the contractions. Each consecutive contraction of the "20-seconds rhythm" is never signaled as a hunger contraction unless the contractions are very strong, in which case they can hardly be distinguished from the moderate contraction of the periodic or "30-seconds rhythm."

Assuming that the "20-seconds rhythm" is an antrum rhythm, and that the stomach contractions cause the hunger sensations, it follows that strong contractions of the pyloric region should cause hunger. Now, such strong contractions of this region of the stomach occur during vomiting, yet vomiting is, to our knowledge, never accompanied by hunger sensations, although epigastric pain and distress may be felt. Of course it is possible that the change in the physiological condition in the central and the gastric nervous mechanisms usually present in vomiting may account for the absence of hunger. The recognition of only an occasional contraction of the "20-seconds rhythm" as a hunger contraction when all the contractions are of nearly uniform intensity is probably due to variations in attention.

The significance of the time relations between the stomach contractions and the hunger sensations.-It was pointed out by Cannon and Washburn that the time relations between the stomach contractions and the hunger sensations might serve to determine the nature of their causal relationship. It is doubtful, however, whether the data secured by the methods so far employed are of much significance as regards this point. Unless the balloon in the stomach completely fills the stomach cavity and the pressure in the balloon is very slight, it is clear that the manometer does not register the very beginnings of the contractions. And on the subjective side we have the fluctuation of attention as a source of error. 
The recognition of the stomach contraction as a hunger pang depends not only on the strength of the contraction, but also on the rapidity of development of the contraction phase; that is, two contractions may indicate equal degrees of shortening of the stomach musculature, but if the contraction phase of one covers a minute or more, while that of the other half a minute or less, the latter contraction only is accompanied by a definite hunger sensation. The stomach may thus exhibit slow tonus undulations of considerable magnitude without any attendant hunger feeling. This relation of the rate of the contraction of the hunger sensation is in accord with one of the general "laws of stimulation," and would seem to strengthen the view that the-sensation is the result of the contraction.

In no instance out of the numerous tests made on man was the hunger felt before the beginning of the stomach contractions. But when the balloon and the manometer are adjusted as delicately as possible, the hunger signal and stomach contractions appear nearly simultaneously. But inasmuch as the manometer probably does not register the very beginning of the contractions, it is evident that some seconds of the contraction phase always precede the hunger feeling.

When the stomach contraction is of moderate strength and hunger sensation of correspondingly moderate intensity, the hunger sensation usually ceases at the height of the contraction, but when the contractions are very strong the hunger sensation persists also during the relaxation phase. In other words, the sensation lags, both at the beginning and at the end of the contraction.

A certain degree of constant or tonic contraction in the empty stomach appears to give rise to a continuous hunger sensation.-This sensation is a somewhat incomplete feeling of tension or pressure in the epigastric region, but usually less intense and less definitely localized than the pangs of hunger caused by the strong rhythmical contractions. This constant epigastric tension may thus be present during an entire hunger period lasting for 15 to 60 minutes or more, but augmented to distinct hunger pangs with each strong contraction. The degree of tonus or constant contraction of the stomach 
during a hunger period differs with the individual man, and this tonus is not in evidence in some of the lower animals. But it is likely that close introspection will reveal uniformly both the longer hunger rhythms ( 15 minutes to $I_{2} \frac{1}{2}$ hours) and the more rapid rhythm (30 seconds) of the hunger pangs.

The stomach contractions give rise to the hunger sensations.-The consideration of the cause of the gastric hunger contractions will be taken up later, but the simpler question of the action of the contractions may be briefly dealt with now. Assuming, for the present, that the stomach contractions give rise to the hunger sensations through the action of afferent nerves from the stomach, in what way does the contraction act as a stimulus to these nerves? Does the hunger sensation arise (I) from the stimulation of nerves in the mucosa; (2) from the stimulation of nerves in the muscular coats and in the connective tissue; or (3) is it due to an intercentral discharge from the Auerbach's plexus to the brain, associated with the motor discharge from the same plexus to the stomach musculature?

As regards the first possibility, the following experiments have been made, with negative results. It would seem that the only way in which contraction of the stomach musculature can stimulate nerve-endings in the mucosa is by mechanical pressure. This may be imitated in the following way on Mr. V. During the period of relative quiescence of the stomach between two periods of strong hunger, when the afferent nerves concerned are in such condition that their stimulation will give rise to hunger, mechanical pressure on the mucosa by distension of the balloon or rubbing the mucosa by the closed end of a test tube never causes sensations of hunger unless these procedures lead to contractions. Mr. V. always stated that he felt these pressures, but the sensations were not like hunger. The objection might be raised against these experiments that the pressure is not sufficiently strong, and, in the case of the test tube, does not touch a sufficiently large area of the mucosa. We admit that a more intense mechanical stimulation of the mucosa could be produced by Pavlov's method on dogs of blowing sand into the stomach by bellows. But we have not felt justified in 
using similar procedures on Mr. V. The methods used do not, of course, produce the strongest possible mechanical stimulation of the mucosa, but these stimulations were sufficient to affect consciousness. They were perceived, but not as hunger sensations. It seems, therefore, highly probable that the afferent nerves in the mucosa are not primarily concerned in the genesis of the hunger sense. They are, however, concerned in the inhibition of hunger.

The hunger sensation seems to be produced by the contractions only. When the empty stomach is normal, strong contractions, however caused, produced a sensation of hunger. Thus, if the balloon in the stomach is rather suddenly distended, this may produce one, two, or three strong contractions of the previously quiescent stomach, and these are recognized as hunger contractions identical with those of the "spontaneous" hunger periods. It seems to us that this experiment constitutes a demonstration of the peripheral genesis of hunger, as the subjective state clearly is induced by the peripheral change. A tracing illustrating this phenomena is reproduced in Fig. I 2 .

But how do the contractions stimulate the afferent nerves in the muscle layers? Contraction in skeletal muscle stimulates afferent nerve fibers in the muscle. But it seems to us that the pain experienced from contractures or "cramps" in skeletal muscles and in the intestines in the case of colic or tenesmus is somewhat different from the hunger pangs, even though pain is inherent in hunger. The difference may be only an apparent one,' due to the fact that the latter pains arouse the memories of previous agreeable experiences with food. Because of the folding of the mucosa and the submucosa into rugae and the changes in the arrangement of the cells in the muscle layers in the stomach during contractions, there must be a great variation in tension on the nerve-fibers in the contracted and in the relaxed condition of the stomach walls. This variation in tension, rather than actual pressure, may constitute the stimulus, so far as the stimulus is a mechanical one. Hunger, therefore, contains elements of kinesthetic sensation as well as pain, the latter predominating in strong hunger. 
We have been strongly impressed by Mr. V.'s ability to recognize feeble stomach contractions as hunger states. Very strong stomach contractions can be recognized as separate hunger pangs by most people, but there are great individual variations in the ability to recognize each individual contraction, unless the contractions are very strong.
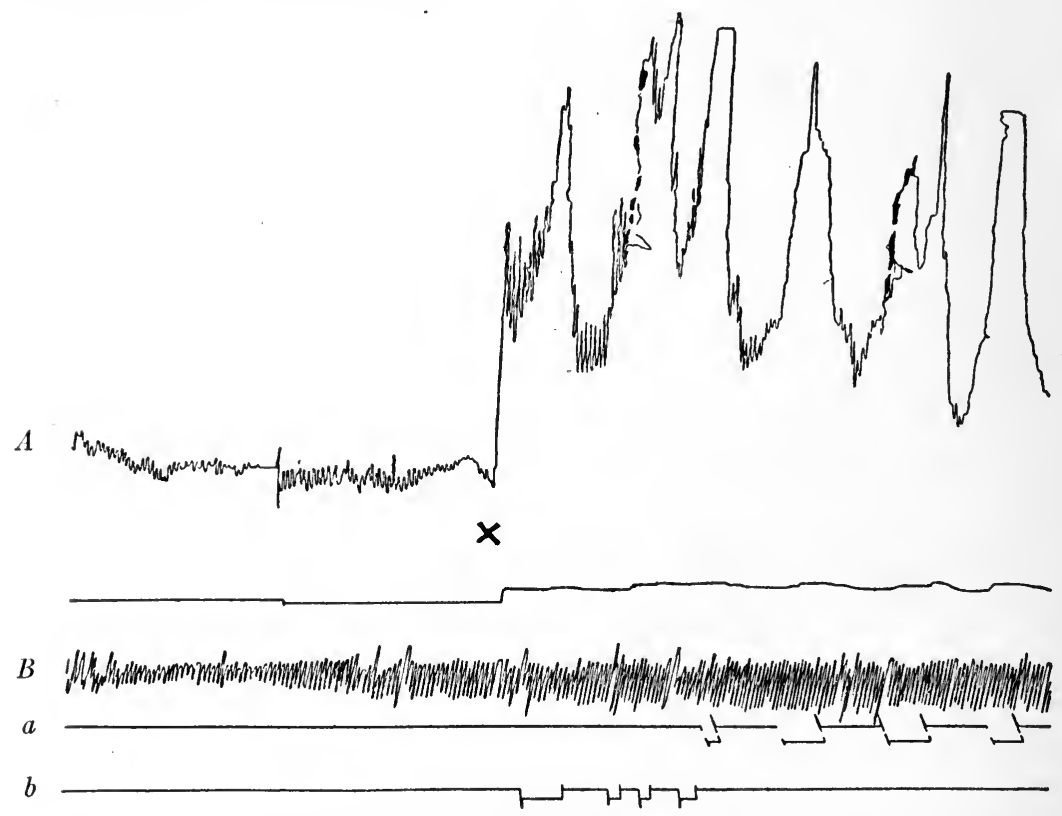

FIG. I 2.-Two-thirds the original size. $A$, stomach contractions; $B$, respiratory movements; $a, b$, signals for moderate and strong hunger respectively. The pressure in the balloon is slight. There is no evidence of strong stomach contractions, and Mr.V. feels no hunger. At $x$ the pressure in the balloon is suddenly increased. This distension of the balloon initiates a few strong stomach contractions, which in turn cause the hunger states. A demonstration of the gastric genesis of hunger.

Two explanations of Mr. V.'s unusual ability in recognizing the stomach activities have occurred to the writer. Since early boyhood the stomach has been to Mr. V. the object of special care and attention. In consequence of such special attention to the stomach the afferent nervous impulses from the stomach may attain a clearer definition in consciousness analogous to the remarkable development 
of analysis in the tactile or pressure senses in the absence of vision. That this type of education or training does take place, the author is satisfied from his own experience during the four years that he has studied the hunger mechanism on himself. At the point of the gastrostomy Mr. V.'s stomach adheres to the parietal peritoneum: There may be adhesions of greater extent in consequence of the operation. The hunger sensation of Mr. V. may therefore include a greater degree of pain than is the case in normal men, as the contracting stomach may pull on the parietal peritoncum, which, according to many observers, is very sensitive to painful stimuli. The weaker stomach contractions may thus be recognized as hunger because more painful than under normal conditions. Confirmatory evidence (or the opposite) ought to be obtainable without much difficulty, as cases of gastrostomy are fairly common. Clinicians having such cases in hand would do a service to physiology if they could determine whether gastrostomy invariably augments the hunger sensations or makes the hunger pangs more painful.

We have shown that in all animals so far studied the empty or partly empty stomach exhibits the tonus and contractions which in man give rise to the hunger sensation. There are some differences in these contractions in different species, but these are probably of minor importance. Manknows that a sensation of strong hunger is felt synchronously with the strong contraction. We have a right to conclude that the lower animals experience the same sensation simultaneously with the corresponding contraction. We have, then, in the tonus and contractions of the empty stomach an objective criterion for the presence or absence of hunger in experimental animals in normal health.

III. THE ESOPHAGUS AND THE CARDIA IN HUNGER

I. METHODS OF INVESTIGATION

There is some evidence in the literature of contractions of the esophagus synchronous with the periods of gastric hunger contractions. Some people refer the hunger sensation or hunger pangs, 
not only to the stomach, but also to the chest and throat. This appears to be true, not only of ignorant people, such as those interrogated by Schiff, but also of persons of special training in. introspection. Cannon and Washburn have described periodic contractions of the lower end of the esophagus in man, and these contractions seemed to give rise to hunger pangs just as in the case of the contractions of the empty stomach. They suggest that the esophagus contractions are synchronous with the gastric hunger contractions, but they did not prove the hypothesis by recording the stomach and esophagus contractions simultaneously.

We studied the motor conditions of the esophagus in hunger on three men who experienced no difficulty 'in swallowing simultaneously the gastric and the esophageal balloons with their flexible rubber tube attachments. In part of the work we used the best quality of rubber condums also for the esophagus balloon, not in their entire length, as in the case of the stomach, but cut down to a length of 3 to $4 \mathrm{~cm}$. We soon encountered difficulties in the work with the esophagus, difficulties apparently not noticed by Cannon and Washburn. Thinking that part of these difficulties might be due to the diameter of the esophagus balloon, we resorted to the rubber finger cot employed by these observers. But even the best rubber finger cots are not as satisfactory as the condum balloon.

The position of the balloon in the esophagus was usually determined by the distance from the incisor teeth to the lower end of the balloon. If the balloon is clear above the cardia the movements of inspiration decrease the positive pressure in the distended balloon in proportion to increase in the negative pressure in the thoracic cavity. But if the balloon is located in the region of the cardia itself, it depends on the relative preponderance of diaphragm and chest movements whether the act of inspiration leads to increase or decrease in the balloon pressure. The esophagus balloon can be well in the cardia and still show negative pressure in inspiration if the inspiration is predominantly costal. On the other hand, if the lower end of the balloon is just within or at the cardiac orifice, a diaphragmatic inspiration increases the pressure in the balloon, 
although not to the same extent as when the balloon is in the fundus of the stomach.

In the case of A. J. C. and J. H. L. it was found that when the distance from the lower end of the esophagus balloon to the incisor teeth was $15 \frac{1}{2}$ to 16 inches the balloon was as far down as it could be located without being directly affected with the contractions of the cardia and the stomach. Allowing the balloon to slip down $\frac{1}{2}$ to I inch farther brought it to the cardia and the cardiac end of the stomach. In the case of A. B. L. the distance from the incisor teeth to the lower end of the balloon could not exceed $I_{4} \frac{1}{2}$ to $I_{5}$ inches, if pure esophagus effects were to be obtained. When the esophagus balloon is located I4 to I6 inches from the incisor teeth, it is obviously well below the level of the heart, and therefore in the region of myenteric plexus and nonstriated musculature of the esophagus.

The esophageal and stomach tubes were usually joined together firmly, so that the lower end of the esophagus balloon was $1 \frac{1}{2}$ inches above the upper end of the stomach balloon. 'The pressure in the esophagus balloon varied between $\mathrm{I}$ and $4 \mathrm{~cm}$. of chloroform.

\section{RESULTS IN MAN}

Local contractions and peristalsis.-When the esophagus balloon is distended with a pressure of 2 to $4 \mathrm{~cm}$. chloroform the esophagus usually exhibits rapid continuous contractions. These contractions are at times quite regular both in rate and amplitude. The total time of each contraction is less than 2 seconds.

In addition to these contractions the esophagus may show contractions of a tonic character. The duration of these contractions varies from a few seconds to several minutes. If these tonus contractions are only moderately strong, the rapid contractions, just mentioned, continue and are superimposed on the former. In some cases the tonus contractions are quite regular in rate and intensity, but this is exceptional.

Both types of contractions appear to be local. They are not related to esophageal peristalsis, although a peristalsis (caused by swallowing) may induce them in a quiescent esophagus. They are 
not related to gastric contractions, for they occur when the empty stomach is quiescent. They may also occur during gastric hunger contractions, but in this case there is no synchrony between the gastric and the esophageal contractions. They are local contractions of the esophageal tube and not peristaltic, because they do not move the distended balloon. But occasional peristaltic contractions of local origin appear during these contractions. This is shown by the pull on the tube if this is fixed to the teeth, or by the downward movement of the tube if it is not fixed. In this way the esophagus balloon is worked through the cardia unless the tube is secured to the teeth. This peristalsis is obviously identical with the "secondary esophageal peristalsis" of Meltzer.

These esophageal contractions do not give rise to hunger and are in no way associated with this sensation. The rapid contractions do not affect consciousness, but more prolonged tonic contractions are felt if they are strong or moderately strong. They are felt, not as hunger pangs, but as a fulness in the chest or throat, as of something having stuck in the throat. This sensation is so characteristic and distinct that there is no possibility of confusing it with hunger pangs of gastric origin.

These contractions are caused by the local mechanical stimulation of the distended balloon. The contractions are more marked the greater the pressure in the balloon, but at times they appear even with a balloon pressure of less than I $\mathrm{cm}$. chloroform. They are most marked immediately after the introduction and distension of the balloon, but they may persist during an entire observation period of 4 to 5 hours if the balloon pressure is above $\mathrm{I}$ to $2 \mathrm{~cm}$. chloroform. We did not observe any decrease in these local contractions during the progress of the experiments, as one might have expected in case there was any appreciable "education" of the esophagus to the pressure of the balloon. Evidently the esophagus mechanisms are so adjusted that local mechanical stimulation causes local contractions interspersed with occasional peristalsis until the stimulus is removed, that is, the material forced into the stomach. The local contractions as well as the secondary peristalsis

- may be reflexes via the medulla, as indicated by Meltzer's observa- 
tions. But the absence of the contractions after section of the vagi does not prove it, at least for the part of the esophagus provided with non-striated musculature and myenteric plexus. It is known that this part of the esophagus responds to local stimulation with local contractions and peristalsis after recovery from the temporary hypotonicity following section of both vagi.

These local contractions of the esophagus were evidently not encountered by Cannon and Washburn, although the only difference between our technique and theirs is the additional balloon in the stomach (and, in consequence, two rubber tubes in the esophagus). Is the local excitability of the esophagus increased by the presence of a distended balloon in the stomach, a rubber tube in the cardia, and a second rubber tube in the esophagus and mouth? All the men used in our experiments showed these contractions. Can it be the esophagus of Mr. Washburn is exceptional? Or did Cannon and Washburn have the esophagus balloon actually located in the cardia, so that their tracings record the behavior of the cardia rather than that of the esophagus proper?

Boldyreff pointed out that the balloon method cannot be used for recording the periodic contractions of the empty intestines, for the reason that the distended balloon causes local contractions through mechanical stimulation. Fortunately this is not true for the gastric fundus, but it is true for the esophagus, at least to an extent to impair greatly the efficacy of balloon method. For when the esophagus balloon is distended sufficiently to register the slightest tonus variations and contractions of the esophagus, the local contractions are most prominent and disturbing.

Contractions synchronous with the gastric hunger contractions.The weaker gastric hunger contractions at the beginning of a hunger period are usually not accompanied by any esophageal contractions. But synchronous with the strong hunger contractions that mark the culmination of a gastric hunger period there is some persistent increase in the tonus of the esophagus and brief contractions simultaneously with the individual stomach contractions. This increased tonus and rhythmic contractions of the esophagus parallel with the hunger activity of the stomach are in 
evidence even when the esophagus exhibits the rapid local contractions described above. The esophagus contractions appear to lag behind the gastric contractions and are as a rule of briefer duration. The frequent culmination of the gastric hunger contractions in a period of incomplete tetanus of varying durations seems to have no parallel in the esophagus in the way of strong and prolonged contractions.

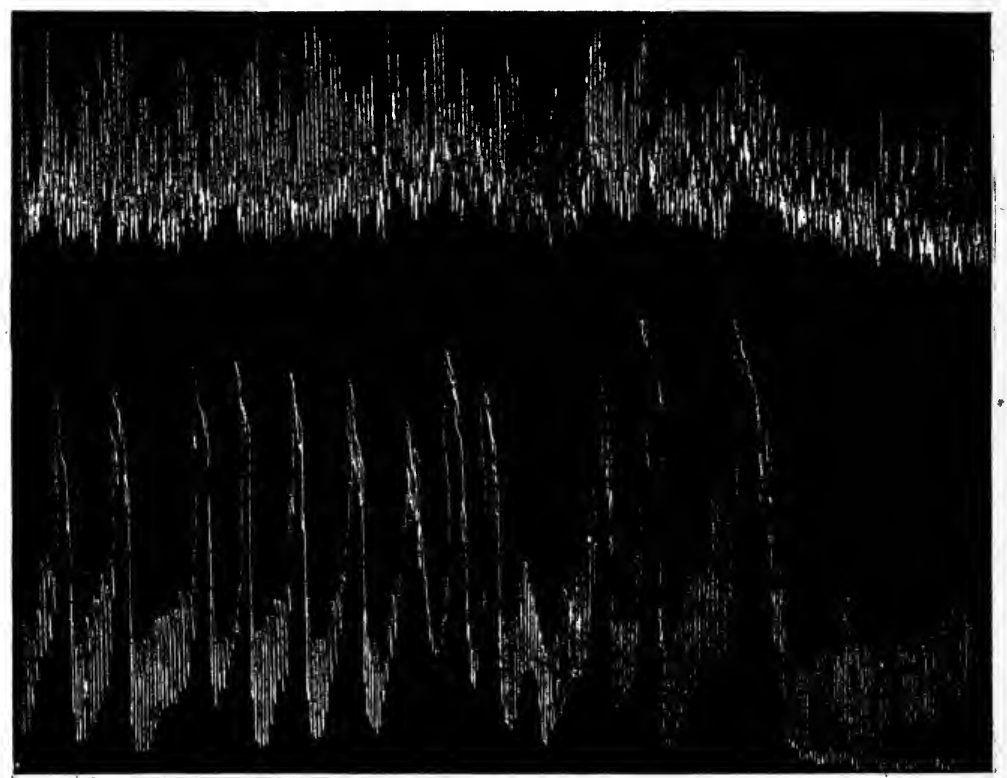

FIG. 13.-Simultaneous records from the stomach (lower curve) and the lower fourth of the esophagus (upper curve) of A. J. C. during the culmination of a period of vigorous gastric hunger contractions. Lower end of esophageal balloon $=16 \mathrm{~cm}$. from incisor teeth, that is, at the cardia. Pressure in balloon $=3 \mathrm{~cm}$. chloroform. Showing weak esophageal contractions synchronous with the gastric hunger contractions. One-half original size.

These esophageal contractions parallel with, the gastric hunger contractions are apparently not identical with the esophageal contractions reported by Cannon and Washburn. These observers noted that the esophageal contractions were more prolonged than the gastric contractions of the same man during other hunger 
periods. 'The contractions noted by us are usually briefer than the parallel stomach contractions. Washburn was able to associate the esophageal contractions with the sensation of hunger pangs. None of us are able to do that. In the first place the esophageal contractions that occur spontaneously during quiescence of the empty stomach contractions that may be identical in rate and strength with those parallel with the gastric hunger contractions are either not felt at all, or else felt as a disagreeable fulness in the throat, something stuck in the esophagus, and not as the uncomfortable emptiness that characterizes the genuine pangs of hunger. To be sure, when the gastric hunger contractions are sufficiently intense to be definitely accompanied by esophageal contractions all of us feel the pangs of hunger strongly, but these are of gastric origin and are referred to the stomach and not to the esophagus or throat.

Contractions of the cardia.-That there is an increase of the tonic contraction of the cardia during the gastric hunger contractions is rendered probable by the fact that the air and other gases always present in the stomach are not forced into the esophagus during these contractions even when they are very strong. Cannon and Washburn state that this fact argues for contractions of the esophagus parallel with the contractions of the empty stomach. Did they not overlook the fact that the cardia is capable of doing this even in the absence of esophageal contractions? There is no escape of air from the stomach during the periods of incomplete gastric tetanus at the end of a hunger period, although these contractions are practically never accompanied by any esophageal contractions. In the dog esophageal contractions are known to be permanently abolished by section of both vagi, yet this does not lead to belching of air even during the greatest increase in intragastric pressure that the contractions of the empty stomach are capable of producing.

It is therefore evident, not only that the cardia itself is able to prevent the escape of air into the esophagus during increased intragastric pressure, but also that the cardia in all probability contracts more powerfully during the gastric 
hunger contractions, thus increasing its efficiency as a guard. Direct graphic evidence of the latter is, however, difficult to secure.

When the empty stomach is quiescent the cardia offers only slight resistance to the withdrawal of a distended balloon of the size of a rubber finger cot from the stomach into the esophagus. A larger balloon, such as the condum used for the stomach, encounters somewhat greater resistance at the cardia, as well as in the esophagus itself. If one attempts to withdraw the balloon from the stomach at the height of a gastric hunger contraction, the resistance offered by the cardia is distinctly increased. This can mean only one thing, viz., an increase in the contraction of the cardia. If the contraction of the cardia did not increase, the withdrawal of the balloon would be actually facilitated by the pressure exerted by the stomach contractions. For example, increasing the intragastric pressure by forcible contraction of the abdominal muscles may force the stomach balloon into the esophagus in case the stomach is quiescent.

It is difficult to keep an inflated balloon actually in the cardia for any considerable time, especially during the strongest gastric hunger contractions. Strong esophageal peristaltic movements keep pushing it toward the stomach, and at times the gastric contractions actually push it back into the esophagus. At the best the balloon will stay in the cardia during two or three successive gastric contractions of the weaker type, that is, at the beginning of the hunger period. The type of balloon used for these tests was the rubber finger cot $3 \mathrm{~cm}$. in length. A balloon of greater length could, of course, be lodged in the cardia with greater ease, but a balloon of greater length than $3 \mathrm{~cm}$. would be influenced not only by the cardia, but also by the cardiac ends of the stomach and the esophagus. In fact this probably occurs, but to a less extent, even when a short balloon is used, as the physiological cardia is probably less than I cm. in width. Anatomically the cardia is not sharply differentiated in man. We judged the position of the balloon in the cardia by the distance of the balloon from the incisor teeth and by the influence of the respiratory movements, moderate inspira- 
tion, mainly costal, causing lowered tension, and moderate diaphragmatic inspiration causing increased tension. When the balloon is in this position the cardia exhibits the 20-seconds rhythm previously reported for the fundus of the empty stomach. This rhythm of the cardia is in evidence even when the empty stomach is quiescent. When the empty stomach shows hunger contractions the cardia shows parallel contractions or periods of increased tonus. The contractions of the fundus and of the cardia are strictly synchronous, but those of the cardia appear to be more persistent or tetanic. The tracings secured by us from the balloon in the cardia resemble those published by Cannon and Washburn as esophageal contractions more than do the actual esophageal contractions obtained by us.

Contractions of the upper end of the esophagus.-In a few experiments the esophageal balloon was placed in the esophagus 7 to ro inches from the incisor teeth, that is, in the lower part of the neck and upper part of the chest. The spontaneous local contractions are in evidence also in this part of the esophagus. There is usually a slight increase of tonus when very strong gastric hunger contractions are present, but nothing like the strength of contractions during the peristalsis of deglutition or those caused by the local mechanical stimulation. The tonus increase of the upper half of the esophagus parallel with the gastric contractions is insignificant compared with the corresponding contractions of the lower third of the esophagus. This is probably correlated with the gradual disappearance of non-striated musculature and myenteric plexus in the oral half of the esophagus in man.

\section{RESULTS ON DOGS}

All of our dogs used in this work were provided with a gastric fistula for the introduction of the stomach balloon. The esophagus was left intact and the esophageal balloon introduced through the mouth. The dog is of special interest in this connection, because the dog's esophagus is composed of striated musculature throughout its whole length. The myenteric plexus is probably lacking. It is needless to say that all the dogs were subjected to preliminary 
training in the way of getting used to the balloons in stomach and esophagus. As a matter of fact all the dogs were accustomed to these procedures, having been used for other lines of work on the hunger mechanism, so that they would lie quietly and comfortably in the lap of an attendant during the tests. It is absolutely essential that the dogs be quiet, if possible sleeping, during these tests, for restlessness increases the disturbance of the esophagus even more than of the stomach.

Local spontaneous contractions.-The presence of the inflated balloon in any region of the esophagus caused rapid local contractions, more rapid than those of the human esophagus, alternating with an occasional peristalsis, also of local origin, and occasionally more prolonged tetanic contractions. These tetanic contractions usually last for only half a minute to a minute. In one case it lasted I 5 minutes. Tetanic spasms lasting up to 5 minutes are not uncommon. These contractions have no relation to the hunger contractions of the empty stomach, as they may appear during a hunger period as well as during gastric quiescence. They depend on the local stimulation of the balloon in the esophagus and are, therefore, more marked the greater the tension in the balloon. They are also more marked when the dogs are excited, evidently owing to increased reflex excitability of the medullary centers and some increase in the tonus of the esophagus. The disturbance is greatest when the balloon is first introduced, but it may keep up for hours even when the pressure in the balloon is only 2 to $3 \mathrm{~cm}$. of bromoform or chloroform. A single swallowing act may induce these local contractions, lasting for many minutes, in a quiescent esophagus. The same thing has been observed in man. It is probably due to increased reflex excitability of the medulla and to increased tonus of the esophagus, as the latter is equivalent to increased tension in the balloon, and therefore increased strength of the local mechanical stimulation.

When the esophagus contracts in tetanus on the balloon, additional disturbing factors appear. Evidently this kind of contraction causes the same sensation in man and dog, that is, the feeling of something stuck in the throat, for when these contractions 
are present the dogs become restless and sometimes swallow repeatedly.

Condition of the esophagus during gastric hunger contractions.The gastric hunger contractions are not accompanied by contractions or changes of tonus in any part of the esophagus. If the gastric hunger contractions are very intense, so that they cause the dog to moan or swallow, contractions may appear in the quiescent esophagus, but this is obviously due to the general disturbance of the animal. There is no synchrony between the gastric and the esophageal contractions. If the dog lies quietly or is sleeping, a strong gastric hunger period may run its course without the least evidence of esophageal contractions.

Inhibition of the gastric hunger contractions during the tetanic contractions of the esophagus.-The strong and prolonged local contractions of the esophagus cause inhibition of the gastric tonus and the gastric hunger contractions, while the rapid esophageal contractions seem to have no effect on the stomach. This is evidently a special instance of the "law of the intestine," that is, inhibition caudal or aboral to a region in the state of contraction. Other factors may also be concerned in this inhibition of the stomach. The tetany of the esophagus causes some distress, and this may lead to inhibitory action via the splanchnic nerves. The gastric inhibition ordinarily lasts as long as the tetanus in the esophagus.

Contractions of the esophagus in response to seeing and smelling food.-Seeing and smelling meat on the part of a hungry dog almost invariably led to contractions of the otherwise quiescent esophagus. Ordinarily the contractions are of the local rapid type, and the disturbance of the esophagus lasts only for to to 30 seconds. But occasionally the sight or smell of food may send the esophagus into complete tetanus lasting for several minutes. Both types of esophageal contractions are accompanied by inhibition of the stomach. A single act of swallowing, as we have seen, may produce the same disturbance in the part of the esophagus where the balloon is located, and the dog frequently swallows or at least elevates the larynx at the sight of the food. But these contractions on the sight of food also appear when the dog does not swallow. The 
phenomenon is probably to be explained as follows: The sight or smell of food on the part of the hungry dog causes temporary increase in neuro-muscular tonus, including the tonus of the esophagus. The increase in esophagus tonus causes greater pressure on the balloon, and in consequence stronger mechanical stimulation of the afferent nerves in the esophagus, and hence the rapid or tetanic contractions. It is therefore probable that the actual contractions of the esophagus on seeing food are artifacts, so to speak, due to the presence of the balloon.

\section{THE INTESTINES IN HUNGER}

Periods of active contraction of the small intestines in hunger were observed in the duodenal fistula case of Busch. Boldyreff found that the intestines (presumably both the large and small intestine) exhibit rhythmic contractions during the periods of gastric hunger contractions. His evidence consisted of the sounds produced by the moving gas in the intestines and the appearance of the free end of the fistula of the small intestine, during the periods of strong rhythmical contractions of the empty stomach. More accurate methods of registration should be devised. We have observed in dogs with duodenal fistula evidences of contractions in the duodenum during hunger contractions of the stomach (expulsion of bubbles of gas, fluid, débris, etc.). There can be no doubt that Boldyreff's observation is correct. In two dogs we introduced balloons both into the stomach and into the large intestine (descending and transverse colon). The large intestine showed some contractions, but these were not correlated with the periods of gastric hunger contractions, although the interesting theory recently developed by Alvarez seems to imply that increased tonus and contractions of the stomach must lead to increased motor activity in the entire gut below the stomach.

Rumbling intestinal sounds may be heard when the stomach is quiescent. These are probably due to local stimulation resulting from distension by the gases, hence mostly confined to the large intestine. Rumbling intestinal sounds are usually heard during gastric hunger periods, and it seems to us, one can at times actually 
feel the intestinal movements. If this is the case, the intestinal hunger movements must be much more vigorous than the digestive movements. The character of the intestinal hunger contractions is not known. Do they consist in peristalsis, in segmentation, or in pendulum movements? Or is it a type of contraction not seen during digestion: rhythmic contractions and relaxations throughout the whole intestine and synchronous with the systole and diastole of the hunger beats of the stomach and the lower end of the esophagus? This would imply a type of co-ordination of the neuromuscular mechanism of the entire digestive tract not revealed by the movements of digestion. In the movements of digestion the esophagus, the cardia, the fundus, antrum pylori, the pyloric sphincter, and the intestines act as relatively independent mechanisms and appear to be governed by laws of their own in harmony with the rôle played by these regions in digestion.

While we admit the possibility that the contracting esophagus and intestines may contribute to the sensation of hunger, the proof for this is still wanting, and in any event the stomach is the main factor. The hunger pangs run absolutely parallel with the periods of gastric hunger contractions, only lagging some seconds both at the beginning and the end of each contraction. A strong contraction artificially induced in the empty stomach is recognized as a hunger pang; a similar contraction induced in the esophagus when the stomach is empty is recognized as something quite different from hunger. And this will in all probability prove to be true also of the intestines. No one can fail to recognize the difference between the sensations initiated by strong contractions in the small and large intestines and the rectum (intestinal cramp, tenesmus, defecation) and the gastric hunger pangs. 


\section{CHAPTER V}

\section{SOME ACCESSORY PHENOMENA OF HUNGER}

Many apparently normal persons experience in hunger, besides the gnawing pressure-pain sensation in the stomach, a feeling of weakness, "emptiness," headache, and sometimes even nausea. A certain degree of increased excitability of the central nervous system, as shown by restlessness, irritability, diminished concentration and attention, and some salivation are always present and must be looked upon as a necessary effect of hunger rather than accessory elements.

We call these states or symptoms accessory hunger phenomena, because they are not always present in hunger, and because their relative preponderance depends on the length of starvation and on some individual peculiarity in the person. It must be admitted, however, that in some individuals these accessory hunger phenomena appear to overshadow, if not entirely to suppress, the pressure-pain sensations from the stomach. This is probably due to a relatively unstable condition of certain central nervous mechanisms rather than to actual absence of the hunger tonus and hunger contractions of the empty stomach, although there are unquestionably great individual variations in the latter, even in comparatively healthy persons. The question can be settled by direct test on persons who claim to feel weakness but no gastric hunger pangs in starvation.

We think most observers will agree that in the normal person the hunger experienced 4 to Io hours after a meal is primarily the gastric gnawing pangs, with practically no feeling of weakness, and no marked or obvious hyperexcitability of the brain and spinal cord. This is the justification for calling the gastric sensation the primary and essential factor in the hunger complex. Let us now consider the cause of the accessory hunger phenomena, and their relation to the gastric hunger pangs. 
I. GASTRIC TONUS AND HUNGER CONTRACTIONS INCREASE REFLEX EXCITABILITY OF CENTRAL NERVOUS SYSTEM

This is readily made out by recording the amplitude of the knee jerk (or any other motor reflex) parallel with the graphic recording of the gastric contractions. Such records show without exception a marked increase in the reflex excitability of the spinal cord simultaneously with the strong hunger contractions of the empty stomach. The reflex excitability usually falls to normal level during the pauses between the single contraction, and after the strong hunger period it appears to be somewhat lower than normal. The hunger contractions of the empty stomach frequently increase the reflex response of the cord to such an extent that a standard minimal stimulus causes a maximal response. The degree of augmentation of the reflex is, on the whole, proportional to the amplitude of the stomach contractions, and this can be shown frequently during the shortening phase of a single contraction. There are many exceptions to this last statement, to be sure. But this is to be expected in view of the fact that under conditions as nearly uniform as possible two successive stimuli of equal character and intensity rarely give two successive responses of equal magnitude.

Some attention was paid to the question whether this augmentation of the reflex excitability of the central nervous system synchronous with the hunger contractions of the stomach is in evidence before the contractions have given rise to the conscious hunger pangs. This much is certain, that the augmentation is greatest at the height of the stomach contraction when the hunger pang is the most intense. The present data do not warrant any statement on the question whether or not the beginning of the augmentation precedes the conscious hunger pang to the same extent that the stomach contraction precedes the hunger pang, because little importance can be attached to slight variations in the amplitude of the reflex response, except when the general average is made significant by the great number of the individual experiments. The stomach contraction is the primary factor or stimulus, whether or not conscious cerebral processes constitute a 
necessary link in the chain of events that results in augmentation of the reflexes.

Lombard concluded from experiments on himself that hunger depresses the knee jerk. No account is given of the degree of hunger experienced by him before lunch and dinner. Possibly he
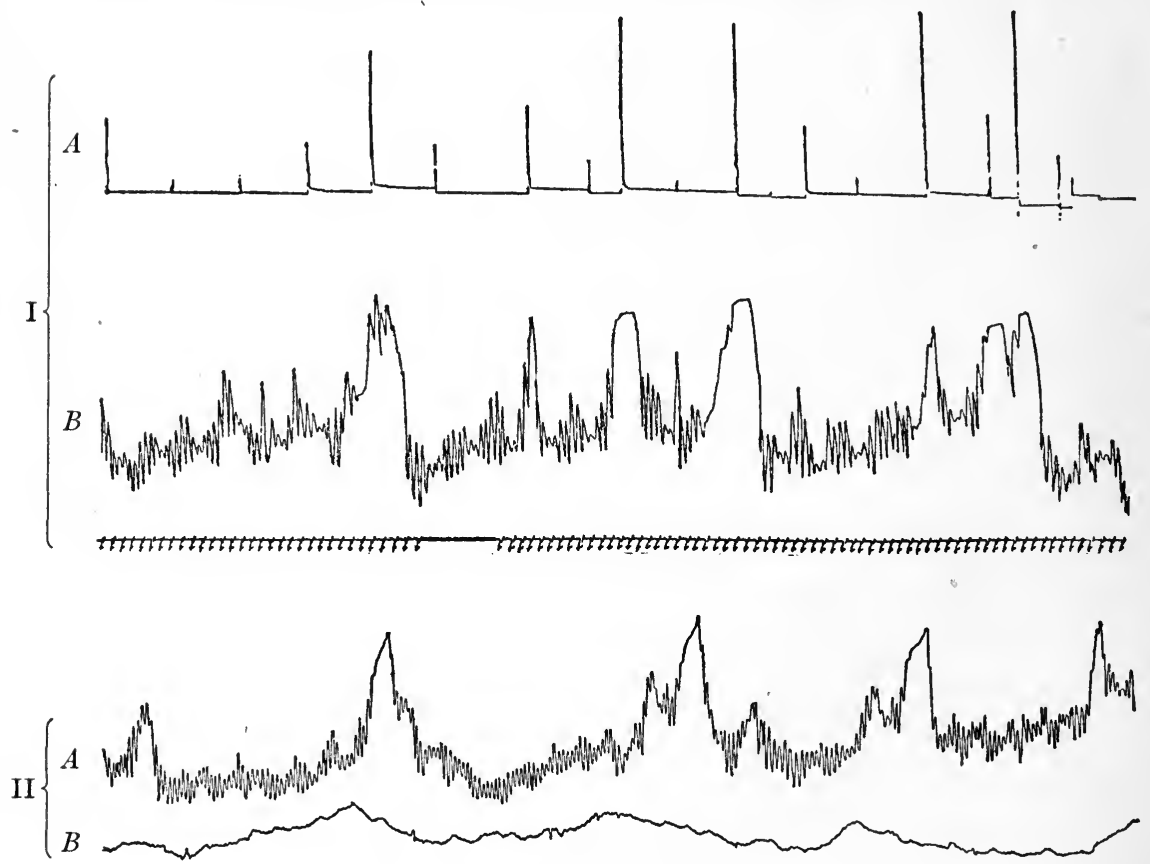

FIG. I4.-I. $A$, record of the knee reflex; $B$, hunger contractions of the empty stomach; showing augmentation of the knee reflex during the hunger contractions. Time, five seconds. About one-half original size.

II. $A$, contractions of the empty stomach; $B$, plethysmograph record of volume of the left arm; showing a vasomotor rhythm parallel with the strong hunger contractions of the empty stomach. About one-third original size.

experienced only appetite and the feeble and "indefinite" hunger that may be present in the absence of the strong stomach contractions. In such conditions there is no augmentation of the knee jerk in our subject. It should also be noted that in some cases Lombard found his knee reflex greater before the meal (hunger?) than after the meal. 
It may be questioned whether a comparison of the amplitude of the knee reflex before and after a meal is an adequate criterion of the effect of hunger states on the reflex excitability of the spinal cord. It seems to us that this is a comparison between hunger (or appetite) and satiety, and not between hunger and the absence of hunger. The partaking of food when hungry involves many changes of a positive character besides the abolition of hunger. Hence it is clear that there is no contradiction between our results and Lombard's results on himself. The two series cannot be compared, because the conditions of the subjects were not comparable. We have made no tests on Mr. V. before or after a meal, similar to those of Lombard. But when the comparison is made between the state of hunger (as differentiated from appetite) and the absence of hunger, the evidence is conclusive that hunger leads to or is associated with an increased excitability of the cerebro-spinal axis. This condition probably accounts for the irritability, restlessness, and inability to maintain a fixed attention-always noted in connection with strong hunger, and in some persons, even in case of moderate hunger. The afferent nervous impulses from the stomach in hunger interfere with other reflex and central processes by monopoly of attention as well as by changes in the reflex centers. According to Weygandt starving persons dream more than usual in their sleep.

Stimulation of the gastric mucosa produces a similar increase in reflex responses. Thus hot or cold water, introduced into a stomach by a tube, so that there is no stimulation of the nerves in the mouth or the esophagus invariably augments the knee jerk in man.

Since the gastric hunger contractions actually induce the increased excitability of the central nervous system reflexly, it follows that this is a necessary phenomenon in hunger. The precise degree of this central effect will depend on the intensity of the hunger contractions, the irritability of the afferent gastric nerves, and the stability of the central organization. The increased reflex excitability and restlessness are, at least in part, subconscious phenomena, since it is in evidence even in animals deprived of their cerebrum (Goltz, Rogers). 
II. INFLUENCE OF GASTRIC HUNGER CONTRACTIONS ON THE HEART AND ON THE VASOMOTOR MECHANISM

I. The heart beat.-During the period of strong stomach contractions the rate of the heart beat is increased. At the cessation of the contraction period the heart rate becomes slower again. The return to the normal rate is gradual. If the motor activities of the empty stomach are very vigorous, so that the pauses between the contraction periods are relatively short, the pulse rate may not return quite to the normal during these pauses. The average increase in the rate of the heart beat during the hunger contractions of the stomach is eight to ten beats per minute. But strong individual contractions or tetanus periods may show an increase of thirty beats per minute.

When the details of the contraction periods are further analyzed, it is found that the greatest acceleration of the heart beat is on the whole synchronous with the individual strong contractions, and that the pauses between the contractions usually show less acceleration. It is not known whether this effect on the heart is a reflex from the stomach, or due to a direct lowering of the vagus tonus.

2. Vasomotor changes synchronous with the contractions of the empty stomach.-It is well known that the vasomotor center is acted upon not only by practically all afferent impulses, but also by the conscious centrifugal impulses from the cerebrum. None of these disturbing factors can be controlled completely. The most we can do is to endeavor to make the external conditions and the cerebral processes as uniform as possible. This was attempted in two ways during these experiments. The subject was permitted to read stories; he was required to add figures, or his eyes were covered and he was instructed to think of nothing in particular. Aside from the varying cerebral states, auditory stimuli are the greatest disturbing factors.

The periods of strong contractions of the empty stomach are synchronous with great variations in the vasomotor tone, and in most cases the vasomotor variations exhibit a rhythm similar to 
that of the stomach. There is an increase in volume of the arm (vasodilation) pari passu with an increasing tonus of the stomach and with the beginning of the individual contractions, but the arm begins to shrink (vasoconstriction) before the stomach contraction has reached its maximum. Or the volume of the arm shows a definite increase parallel with the strong contractions, and a corresponding diminution in the arm volume during the stomach pauses. These two types of vasomotor rhythms were the ones usually obtained during periods of moderate hunger contractions. If the experiments were continued for long periods ( 4 to 6 hours), so that the hunger contractions and hunger tetanus became very strong, the vasomotor variations were in evidence, but the rhythms were rarely synchronous with the stomach rhythms. In such instances the subject gave unmistakable signs of restlessness. Under these conditions the vasomotor rhythm may be slightly faster or slightly slower than the stomach rhythm, or the two rhythms may be practically identical, but the contraction phase of the stomach activity may be synchronous with a decrease in the volume of the arm (vasoconstriction).

Before the appearance of the first period of hunger contractions after a meal, and during the pause or relative quiescence of the stomach between the periods of moderate hunger contractions, another type of vasomotor rhythm appears in the plethysmographic records. Considerable attention was given to this rhythm, because the rate of it suggested some correlation with the "20-seconds rhythm" of the empty stomach. These slight variations in the arm volume are frequently irregular, so that its rate cannot be made out with certainty, but when the fluctuations are fairly regular the rate corresponds closely to the "20-seconds rhythm" of the empty stomach. The contraction and relaxation phases of the two rhythms do not seem to correspond, but that is of little significance in view of probable difference in the latent time of the respective neuro-muscular apparatus as well as of the recording devices. This type of vasomotor rhythm is most marked when the "20seconds rhythm" of the stomach is the strongest, that is, at the beginning of a period of strong hunger contractions. 
In view of the fact that the vasomotor center is acted on by so many factors, central and peripheral, the parallelism between the vasomotor tone and the motor activity of the empty stomach during the hunger period is too regular to be accidental. When the synchrony fails, this is probably due to central or peripheral factors that cannot be controlled or recorded. The question, then, remains how this synchrony is brought about, and what is the biological significance of it. (I) The co-ordination may be due to associations between the vasomotor center and the center for gastric tonus in the medulla. (2) It may be due to a direct action on the vasomotor center by afferent impulses from the stomach initiated by the stomach contractions. (3) Or, it may be due to the influence on the vasomotor center of the conscious processes of hunger, which are caused by the stomach contractions. Brodie and Russel, and Miller have studied the effect on the blood pressure of stimulation of the central end of the gastric branches of the vagi in animals under general anesthesia. The changes in the blood pressure are variable and complicated by respiratory changes and vomiting movements. In dogs and cats the primary effects may be either an increase or a decrease in the arterial pressure, while in rabbits the stimulation seems to cause a rise in the blood pressure only. It will probably be difficult to secure experimentally the selective stimulation of the afferent gastric nerve fibers that are stimulated by the contraction of the empty stomach. There may be some connection between the vasomotor rhythms described above and the well-known Traube-Hering blood-pressure variations, as the latter are frequently induced by experimental interferences with the vagi.

\section{SALIVATION}

When a suitable cannula is put in the Stenson's duct in man so that the rate of flow of the saliva can be measured accurately, and with a balloon in the stomach to register the gastric hunger contractions, one can demonstrate that there is a rhythm of the salivary flow parallel to the gastric hunger-contraction rhythm. Each strong hunger contraction is accompanied by a brief gush of 
saliva from the duct. The amount of salivation varies with the individual person, and it varies somewhat from day to day in the same individual. The feebler contractions have no evident effect on the saliva flow. This saliva flow is independent of the memory, sight, or smell of palatable food during the hunger, but it is certain that these stimuli and the central processes induced by them will augment the salivation.

The increased flow of saliva that occurs simultaneously with the strong hunger contractions is probably a reflex effect from stimulation of sensory nerves in the stomach by the contractions. It is well known that strong stimulation of gastric nerves induces salivation and vomiting. Such stimulation is usually held to act on the nerves in the mucosa, while the stomach contractions stimulate the nerves in the muscularis mainly. It is probable that strong stimulation of the sensory nerves in the sub-mucosa and in the muscular layers also causes reflex salivation.

Some increased salivation is therefore a necessary effect of strong hunger, irrespective of the presence of appetite, or the memory, sight, or smell of good food. But the more copious "watering of the mouth" that accompanies the thought or sight of appetizing food is not of gastric origir.

\section{EFFECTS ON THE VASOMOTOR CENTER OF STIMULATION OF THE GASTRIC MUCOSA}

When cold or hot water is introduced into the stomach through the tubes in quantities of 50 to roo c.c. at a time, there is nearly always a shrinking of the arm, that is, vasoconstriction. Water at body temperature does not seem to affect the tonus of the vasomotor center. Water at $10^{\circ} \mathrm{C}$. causes less vasoconstriction than water at $55^{\circ} \mathrm{C}$.

In order to ascertain whether this reflex is due to the influence on the vasomotor center by conscious processes, similar experiments were performed while the subject was asleep. It is not an easy matter to go to sleep with a plethysmograph on one arm and two rubber tubes in one's throat and esophagus. But, after marked physical fatigue it is possible. The same rhythmic variation in the 
vasomotor tone is observed as before, but perfectly relaxed. There is a gradual increase in the size of the arm as the tonus of the stomach increases. This increase or vasodilation is greatest when the stomach contractions are highest and the size of the arm decreases as the stomach relaxes. From this it seems evident that this activity of the vasomotor center is not due to conscious processes.

Moderately hot or moderately cold water in the stomach does not seem to affect the activity of the vasomotor center during sleep. Very cold water either does not have any very marked effect, or else the stimulation is sufficient to awaken the subject. Warm water did not cause a marked vasoconstriction unless the subject woke up as a result of the injection. The marked vasoconstriction observed when the stomach mucosa is stimulated with very cold or very warm water, therefore, seems to be due mainly to the effect of conscious processes on the vasomotor center.

V. FEELINGS OF WEAKNESS, "EMPTINESS," HEADACHE, AND NAUSEA IN HUNGER

I. The weakness accompanying hunger is evidently of complex origin, or partly due to sensory impulses from the digestive tract, and partly to relative exhaustion in the tissues. Voit thought that the feeling of weakness is a general sensation in some way caused by lack of nutrient materials in the blood. That in moderate hunger only the first or reflex factor is involved is evident from the fact that this weakness is abolished by taking food into the stomach, before there is any digestion and absorption of the food material into the blood. After several days of starvation, the feeling of weakness does not all disappear immediately following a meal. Hence we must here be dealing also with the factor of replenishing the tissues. How does the hunger state of the stomach and intestines induce the feeling of weakness? We have seen that this hunger state means strong tonus and contractions. of the empty stomach and intestines. It probably also involves a greater degree of tonus of the abdominal muscles in the maintenance of the intraabdominal pressure. It is not clear, however, why tonus and 
contractions in the empty stomach should in some people produce the feeling of weakness.

We are not convinced that the vasomotor disturbances caused by the hunger contractions are great enough to produce the feeling of weakness. There are probably other reflex factors at present unknown. At the same time, the degree of weakness caused by identical peripheral factors evidently depends on the stability of the central nervous organization, for in disease (hysteria, neurasthenia, etc.) a degree of hunger which, in a normal person, would not induce the feeling of weakness may cause extreme prostration.

2. The feeling of "emptiness."-Strong sensation of hunger is usually accompanied by a peculiar feeling of "emptiness" in the entire abdominal region. This feeling is continuous, not intermittent like the pangs of hunger. The "emptiness" feeling does not disappear entirely during the relative quiescences of the empty stomach between the hunger periods.

This feeling is probably more complex in its origin than the hunger pangs. It is well known that a very complete evacuation of the large intestines, as by enemas or purgatives, induces a feeling of emptiness. The feeling may be partly abolished by moderate but steady pressure on the abdominal wall. It is probable that the increased tonus of the abdominal muscles, in consequence of the empty state of stomach and intestines, contributes to the feeling in some way. It is well known that the intra-abdominal pressure remains nearly constant under varying degrees of fulness and emptiness of the digestive tract.

Since in starvation there is persistent hypertonus of the abdominal muscles, we question whether any part of the feeling of emptiness originates in the stomach itself. It is not so difficult to understand how a hypertonic and rhythmically contracting empty stomach may give rise to sensations of tension, pressure, and gnawing pain. But how can it cause the sensation of emptiness, unless this feeling is merely the negative of the sensation of fulness? On the other hand, if the tonus of the abdominal muscles does not suffice to maintain the normal intra-abdominal pressure 
when stomach and intestines are relatively empty and strongly contracted in hunger, the tension on all the visceral organs would be diminished, and this in turn would alter the pressure relation to the peritoneum and the mesentery. If this is a factor in the origin of the feeling of abdominal "emptiness" in hunger, the sensation should be diminished in man by lying down, in comparison with that felt when standing. The writer cannot test this hypothesis on himself, as the feeling of "emptiness" is not a prominent part of his hunger complex.

3. Headache and nausea.-The author experiences slight headache and a suggestion of nausea only after 4 or 5 days' starvation, but there is no doubt that a few apparently normal people experience both, or more particularly the headache, during the moderate hunger that comes on 4 to 6 hours after eating. Some of Boring's subjects had difficulty in distinguishing between hunger and nausea in their own consciousness. In such cases there can be no starvation change in the blood and tissues. And the fact that headache as well as nausea is greatly relieved at once by taking food into the stomach seems to show that they are essentially of gastric or reflex origin. It is well known that both headache and nausea can be produced by disturbances in circulation, and that nausea can be caused by stimulation of nerves in the stomach mucosa (normal stimulation of hyperexcitable nerves, or excessive'irritation of the normal nerves). Possibly the strong hunger contractions of the empty stomach cause sufficient stimulation of the gastric nerves to induce both headache and nausea in certain individuals. This can be determined experimentally on such persons. Unfortunately, the author does not himself experience these accessory hunger phenomena, nor has he been able to secure as subject a person with these hunger symptoms sufficiently marked to make it worth while investigating. In prolonged starvation there is a contributory factor, namely, a greater degree of increased excitability both of the gastric nerves and of the central nervous system.

So far as established facts permit conclusions or point the way, the accessory hunger phenomena, excepting the exhaustion fatigue 
of prolonged starvation, are caused reflexly by the hunger tonus and the hunger contractions of the empty stomach. The degree with which they are manifested in hunger depends on the intensity of the hunger contractions, the excitability of the sensory nerves of the stomach, and on the relative stability of the central nervous organization of the individual person. 


\section{CHAPTER VI}

\section{THE RELATION OF HUNGER TO APPETITE}

Physiologists, psychologists, and clinicians who have devoted no special attention to the nature of hunger and appetite appear to accept the common view of the laity that the two sensations are the same in quality, and differ only in intensity. Thus mild hunger is called appetite, while strong appetite is called hunger. If the two sensation complexes are in a linear series and thus involve identical mechanisms, either the term appetite or the term hunger is superfluous, and it would be more correct and less confusing to speak of mild and strong appetite or mild and strong hunger. We do not apply different terms to the sensation of sweetness or of red according to the intensity of the sweet sensation or the saturation of the red color. A sensation remaining identical in quality but differing in intensity should have only one name, and the intensity variation should be denoted by the usual adjectives.

Most authors who have given some special attention to hunger and appetite take the position that the two sensations are fundamentally different, that is, that they differ in quality. This view is supported by Albu, Boas, Boring, Cannon, Carlson, Krehl, Nicolai, Roux, Sternberg, Stiller, and many others. Stiller thinks that appetite is the sensation of hunger plus the memories of taste and smell of foods; hence one may experience hunger without appetite, but not appetite without hunger. But others maintain that one may also experience appetite without the sensation of hunger, as in the case of eating palatable desserts at the end of a full and satisfying meal.

Practically all writers who have recognized the qualitative difference between hunger and appetite state that hunger is primarily an unpleasant and painful sensation and more or less intermittent, while appetite is essentially pleasant in character and without definite periodicity. Hunger, again, is referred to the 
stomach, while the appetite complex is referred to the mouth and throat.

We have seen that hunger is an uncomfortable pain sensation caused by stomach contractions. Is appetite also a sensation, or is it essentially a memory process? If appetite is or contains immediate sensory elements, what mechanisms in the mouth and throat initiate the sensory impulses? In one of his earlier papers Sternberg suggested that appetite is in some way associated with the tonus of the muscles of mastication and deglutition; and that absence of appetite, that is, nausea, is due to atony of these muscles. Later Sternberg suggested that appetite is caused by peristalsis of the esophagus and stomach, while lack of appetite is due to antiperistalsis in these organs. This appears to us a very superficial suggestion. It is logical, to be sure, but it is contrary to fact. In the very full stomach there is active peristalsis, but that does not necessarily cause appetite. And there is no peristalsis in the esophagus except that induced by swallowing. If esophageal and gastric peristalsis are the stimuli that initiate appetite, this sensation should show a rhythmicity similar to that of hunger.

Sternberg, and after him Vorkastner, and others are guilty of a singular misrepresentation of Pavlov's conception of appetite. These German writers charge Pavlov with saying that "Appetit ist saft"-appetite is gastric juice. We cannot find any such statement in Pavlov's work, and the view is obviously inconsistent with the whole trend of his research on the work of the digestive glands. Pavlov has shown that appetite is a necessary condition for the secretion of appetite gastric juice, but appetite by itself does not induce the secretion, nor does the gastric juice by itself induce appetite, although it may contribute to it by gentle stimulation of the gastric mucosa.

According to Cannon, "appetite is related to previous sensations of the taste and smell of food. These sensory associations determine the appetite for any edible substance, and either this memory or present stimulation can arouse the desire." In brief, appetite is caused either by the immediate taste and smell of 
palatable food, or induced by the memory processes of such taste and smell sensations.

Some of Boring's subjects described "a kinesthesis in the throat and oral sensation arising from the free flow of saliva, a complex which to them meant desire for food. Here we have a true sensory • basis of appetite. The ideation of food is no doubt a usual concomitant, and presumably it often constitutes a desire for food that lacks sensory components entirely." Boring thus includes in appetite the sensations aroused by increased salivation, and possibly from increased tonus in the muscles of mastication and deglutition, but not the taste and smell sensations, to which Cannon gives such a prominent position. It may be noted, however, that salivation or increased tonus either singly or combined do not by themselves induce the sensation of appetite.

The delineation of the appetite complex and its relation to hunger is a question of analysis of one's own sensation under such experimental control as can be applied. Now, it is obvious that neither the sight, the taste, nor the smell of good food, the memory of these sensations, or salivation and throat kinesthesis can by themselves invariably induce appetite. We all know that the taste or smell of the best of food in the case of severe gastritis -induces, not appetite, but nausea. Salivation induced by a drop of vinegar in the mouth does not cause appetite. And in nervous anorexia all of the immediate stimuli and the memory processes mentioned by Cannon and Boring may be present or called forth without producing a desire for food. It is thus clear that a certain sensation complex from the viscera and an approximately normal state of central correlations constitute a necessary background for the development of appetite. Given this background, the central and essential element in appetite is the memory processes of past experience (sight, smell, taste) with palatable foods. These memories are reinforced by present stimulation of these nerves by the food, since everyone knows that appetite, unless intense at the outset, is increased by the very act of eating.

It will be shown that part of this augmentation is due to chemical stimulation of the nerves in the gastric mucosa. The mouth and 
throat kinesthesis and the sensation due to salivation are, in the author's judgment, accessory elements in appetite analogous to the sensations of weakness and emptiness in hunger.

But how can we account for the desire for food that seems to be inherent in the appetite elements? We have suggested elsewhere that this urge may be an inherited (partly subconscious) factor (positive chemotropism), fusing with the memory processes of taste and smell of foods as soon as these become a part of the individual's experience. It seems that this urge is present in appetite even when there is no call for food on the part of the empty stomach; hence it is not a vague hunger. Pleasant sensations of different kinds, such as are induced by works of art, music, or the beauties of nature do not seem to contain or arouse analogous desires. In the author's experience the fragrance of the rose in the garden is as pleasing as the fragrance of the roast in the kitchen, but the desire to enjoy. the former cannot be compared with the urge to ingest the latter.

It seems to us that the old view that hunger and appetite are different intensities on the same sensation curve is no longer tenable even as a theory. The conception came about through lack or difficulty of analysis of all factors involved, and the tendency to fuse or confuse in consciousness heterogeneous sensations that are usually aroused simultaneously, as is the case with the taste and smell of food. In the normal individual hunger and appetite are usually experienced simultanèously. If only vague or mild hunger is present, the appetite elements occupy the high seat in consciousness; when hunger becomes markedly painful, attention is focused on this element. If we start to masticate palatable food or by means of a stomach tube put a liquid or liquid food directly into the stomach at the height of a period of gastric hunger contractions, the latter are inhibited at once and the unpleasant hunger sensation disappears at the same time, while the pleasant appetite complex is initiated or intensified. In this way we institute a successive contrast between hunger and appetite, so that they can be compared with greater accuracy.

In everyday life of adult persons having access to a liberal food supply the memory elements of appetite are probably a greater 
factor in the ingestion and digestion of food than hunger, as short intervals between large meals do not permit the development of strong hunger unless the individual is engaged in hard physical work. Under these circumstances appetite and habit supplant hunger as nature's dietary guide. Everyone knows that if the food is made sufficiently palatable we may consume large quantities of it without being hungry or actually needing the food, especially if one has formed the habit of giving marked attention to the pleasures of the table. 


\section{CHAPTER VII}

\section{THE SENSIBILITY OF THE GASTRIC MUCOSA}

The active controversy concerning the sensibility of the visceral organs that has been going on since the time of Haller is essentially a question to what extent afferent nervous impulses from the viscera influence conscious processes. Haller thought the visceral organs were insensitive, especially to pain. Afferent or sensory nervefibers are distributed to all the internal organs. But the stimulation of these nerves may produce local reflexes only, or central reflexes of subconscious character (tonus, vascular, respiratory, etc.). Lennander, a surgeon, observing numerous patients in abdominal operations, under local anesthesia for the abdominal wall, reached the conclusion that, excepting the parietal peritoneum, the entire viscera (stomach included) is insensitive, especially to pain. If Lennander's view is correct the hunger pains cannot be of gastric origin. We presume Lennander would ascribe them to mechanical tension or pressure on the parietal peritoneum from the strong contractions of the empty stomach. Lennander's assistant, Nystrom, and MacKenzie, Becher, and Mitchell have accepted and attempted still further to substantiate and defend this radical theory. But other workers have shown that it is untenable. Kast and Meltzer obtained distinct evidence of pain on injury or strong stimulation of the viscera, and showed, moreover, that local anesthetics (subcutaneous injection of cocaine) tend to depress visceral sensibility. Kast and Meltzer, and Ritter suggest that this accounts for Lennander's erroneous conclusion, as local anesthesia was used in practically all his patients. New experimental facts as well as critical considerations demonstrating certain sensibilities of all the visceral organs have also been published by Neumann, Hertz, Head, Rivers and Sherran, Boring, and others. But in the present work we are .concerned with the sensibility of the alimentary canal, and especially that of the stomach. 


\section{ABSENCE OF PAIN SENSATION FROM THE NORMAL GASTRIC MUCOSA}

The sensation of pain cannot be produced from the normal gastric mucosa except by stimuli that evidently cause some destruction of cells and nerve-endings, such as strong acids, oil of mustard, absolute alcohol, excessive heat, etc. The normal mucosa can be pressed or squeezed with forceps until crushed, pins may be pushed into the mucosa anywhere, or the mucosa may be incised with a scalpel, without causing pain in a normal person. The gastric pain caused by strong and destructive chemicals acting on the normal mucosa may be due to over-stimulation of nerve-fibers other than those of "protopathic" pain. In the case of a hyperexcitable stomach mucosa, as in certain types of gastritis, substances normal to the stomach cavity (water, gastric juice) may cause pain. Strong tonus and contraction of the stomach are painful, and it is not certain that the kinesthetic pain from gastric contraction is excluded in all cases of pain from destructive chemical stimulation of the normal mucosa, or normal stimulation of the hypersensitive mucosa, since the motor conditions of the stomach in these states have not been studied sufficiently. Hertz supports the view that all so-called gastric pains, even those induced by destructive chemical stimulation of the mucosa, are due to excessive contractions of the pylorus and the pyloric end of the stomach. There can be little doubt that strong acids on reaching the duodenum will cause temporary spasms or tetany of the upper part of the duodenum, and probably of the pylorus. The precise genesis of these pains must still be considered an open question. In our own experience these pains are too persistent to be due to peristalsis. It they are of purely muscular origin, the stomach and duodenal contractions giving rise to them must be prolonged tonus or tetany contractions. It is probable that the pain is essentially muscular, but the injured mucosa may also be a factor.

Excessive distension of the stomach by food, water, gas, or inflated balloons causes pain referred to the stomach, but there is no evidence that this pain is due to stimulation of the mucosa nerves. 
We are thus forced to the conclusion that the only physiological pains from the stomach are the pangs of hunger, and these do not arise from the mucosa nerves. All pains possibly originating in the mucosa are indices of pathological processes, that is, either destructive stimuli acting on the normal nerve, or normal stimuli action on hyperexcitable nerves. These gastric pains will be considered in greater detail in the chapter on hunger and appetite in disease (chap. xvi).

\section{THE ABSENCE OF TRUE TACTILE SENSIBILITY}

Everyday experience tells us the stomach mucosa is not sensitive to touch. Pavlov states: "It can hardly be doubted that under normal conditions the surface of the stomach has a certain degree of tactile sensibility." The term tactile sensibility is evidently used here in the sense of a general response to mechanical stimulation rather than as implying a true tactile sensibility. When solid food is swallowed, no tactile sensation is felt after the food has passed the pharynx, unless the mass is so large that it causes unusual distension of the esophagus. In that case it may be felt all the way down the esophagus, but this is due to the distension of the wall of the esophagus and is not a tactile sensation from contact with the mucosa.

Hertz tested on himself the tactile sensibility of the mucosa of the esophagus' by means of an esophagoscope with a slit down the side, so that a metal bulb in a long holder could be moved along the mucous membrane of the esophagus and the pharynx. The pharyngeal mucosa was found to be sensitive to touch; the esophageal was insensitive.

The author has tested the tactile sensibility of his own gastric mucosa, swallowing a good-sized rubber tube, through which was passed a test-tube brush attached to a strong piano wire. Pulling the test-tube brush about in the cavity of the stomach produced no sensation.

Numerous tests were made on the gastric mucosa of Mr. V., our gastric fistula case. Gently touching or striking the mucosa with blunt objects produced no sensation. If the mucosa is rubbed 
or pressed very vigorously with a blunt object Mr. V. says he "can feel it"; he cannot describe the sensation thus produced except in a negative way. It is not like touch, nor is it pain or hunger. Whatever the character of the sensation may be, we are satisfied that it is a real change in the stream of consciousness, for he recognizes these stimuli when he has no other means of knowing that the gastric mucosa is being handled. The sensation may not originate in the mucosa, but in the muscularis (tonus relaxation through reflex inhibition) or possibly in the visçeral peritoneum, as the pressure must be considerable to produce it. We are satisfied that the stimulation of the normal gastric mucosa of $\mathrm{Mr}$. V. does not produce tactile sensation. The same conclusion has been reached by previous workers using human gastric fistula cases.

\section{GASTRIC TEMPERATURE SENSATIONS}

In 1846 Weber suggested that the sensation of cold or warmth in the epigastrium, after drinking ice-cold or very warm water, originates in the skin of the abdomen over the stomach and not in the stomach mucosa. According to Weber sufficient conduction takes place through the walls of the stomach and abdomen to stimulate the temperature nerves of the skin. Becher swallowed a single rubber tube and through it injected water of different temperatures into the stomach. He did not experience any heat or cold sensation before the heat or cold had passed through the walls of the tube and stimulated the mucosa of the esophagus. Mueller concluded that ice water produced no sensation in the stomach. Zimmerman irrigated his stomach with hot or cold water through a thick rubber tube. He claims that hot or cold sensations were felt only when the lower end of the tube was 30 to $25 \mathrm{~cm}$. distant-hence not far from the lower end of the esophagus. He therefore concludes that the sensation is projected from the esophageal mucosa. MacKenzie believes that the temperature sensations induced by hot and cold water entering the stomach is due to reflex vasomotor changes in the skin of the abdomen. Nystrom reports that touching the gastric mucosa of a man having gastric fistula with a hot piece of metal or piece of ice, does not 
call forth any definite temperature sensations. According to Hertz the hot or cold sensations felt in the epigastrium, upon swallowing hot or cold water, come from the lower end of the esophagus. The water accumulates here before the cardiac orifice opens, and that part of the esophagus is thus stimulated more than the parts above. He defends his conclusion by this observation: by auscultation over the epigastrium after swallowing a mouthful of very hot or cold water, one will find that immediately after the second deglutition sound, which occurs after the last trace of food has entered the stomach, the hot or cold sensation disappears. He also states that upon injecting hot or cold water through a double rubber tube, no temperature sensation was noticed before 3 or 4 ounces had passed into the stomach. An ill-defined temperature sensation was then experienced, but he concludes that this was due to the conduction of heat or cold to the esophageal wall. Hence, according to Hertz, the stomach mucosa is not endowed with heat or cold nerve-endings. Quincke introduced hot and cold water into the stomach of a boy with a gastric fistula and reports that the patient experienced vague heat or cold sensations. Neumann and Roux, injecting hot and cold water into the stomach through a double rubber tube, experienced hot and cold sensations in the stomach, the cold sensations being the more pronounced. Head, Rivers, and Sherren injected water at different temperatures into the colon of a patient, upon whom a colostomy had been performed, and found that water at $20^{\circ}$ to $40^{\circ} \mathrm{C}$. produced no sensation at all, but water at $50^{\circ} \mathrm{C}$. and very cold water did give rise to temperature sensations, but that these sensations were by no means as pronounced and as easily localized as those produced by applying water at the same temperatures to the skin. Head concludes that the viscera is endowed with "protopathic" temperature sensibility only.

Boring reports that water at $40^{\circ} \mathrm{C}$. produces a sensation of warmth, and water at $30^{\circ} \mathrm{C}$. a sensation of cold referred to the stomach. In his first paper Boring inclined to the view that these sensations originate not in the stomach but in the abdominal wall; in the second paper he concludes that they arise either in the stomach 
itself or in tissues closer to the stomach than the abdominal wall and the esophagus.

It is thus evident that by far the majority of those who have investigated this problem have experienced a vague hot or cold sensation, in the region of the epigastrium, upon stimulating the stomach mucosa with hot or cold water. Those who do not believe that the sensations arise in the stomach mucosa explain its origin in one of the following ways: (I) It is due to conduction to the skin of the abdomen. (2) It is due to reflex vascular changes of the skin of the abdomen. (3) It is projected from the esophagus. (4) It arises in the lower part of the esophagus.

Our own experiments on Mr. V. and on ourselves go to show that the above-mentioned theories are untenable. That is to say, we find that the gastric mucosa is endowed with protopathic temperature sensibility.

The first series of experiments was on Mr. V. He was blindfolded and was not told the nature of the experiments. The water, 40 to 60 c.c., was injected through a small rubber tube which was passed through the large permanent tube of the fistula. With water at $50^{\circ} \mathrm{C}$. he said that he felt a hot sensation in the stomach, after a latent period of about 5 seconds. When the same amount of water at $10^{\circ} \mathrm{C}$. was injected, a cold sensation was felt after a slightly shorter latent period. A metal rod $5 \mathrm{~mm}$. in diameter, heated to about $50^{\circ} \mathrm{C}$., was passed down through the fistula catheter until the end touched the mucosa of the dorsal wall of the stomach. A longer latent period elapsed here before any sensation of heat was noticed. When, however, a small piece of ice, held by a pair of forceps, was brought into contact with the stomach mucosa, the latent period was not much longer than when cold water had been injected. A cold sensation was distinctly felt. These experiments were repeated over and over again, and Mr. V. was invariably able to recognize whether the mucosa was being stimulated with hot or cold media, provided the media was 45 to $55^{\circ} \mathrm{C}$. and the cold at least $13^{\circ} \mathrm{C}$.

The temperature sensations initiated by touching the gastric mucosa directly with hot or cold solids cannot come from the 
stimulation of the esophageal mucosa. The latent period is too short to allow temperature conductions to the skin of the abdomen. But since it is possible that the cardia and the lower end of the esophagus of $\mathrm{Mr}$. V. are patent, water injected through the fistula may reach the lower end of the esophagus. This possibility was guarded against in a second series of experiments, where we injected the hot and cold water into a very delicate rubber balloon previously introduced into the stomach. The hot and cold water was correctly recognized as hot or cold, just as in the test with the water touching the mucosa directly, the only difference being a somewhat longer latent period. It is clear from the above that the gastric mucosa of $\mathrm{Mr}$. V. is endowed with protopathic temperature sense.

A third series of experiments was carried out on the author and on one assistant (Mr. B.). Three rubber tubes of suitable size were placed one inside the other so as to have three walls of rubber and two air spaces between the liquid in the inside tube and the esophageal mucosa, for the purpose of retarding heat conduction. The inside tube had a diameter of $3 \mathrm{~mm}$. The subject was blindfolded and 50 c.c. of water at $50^{\circ} \mathrm{C}$. were injected into the stomach. A sensation of heat was noted about Io to 15 seconds after the injection, and about 60 seconds before enough conduction had taken place to cause the tube to feel warm in the mouth and throat. When the same amount of water at $10^{\circ} \mathrm{C}$. was injected, a definite cold sensation was felt from 5 to ro seconds after the injection had commenced and about 60 seconds before the tube began to feel cold to the fingers or to the throat.

Both of us experienced hot and cold sensations and never made the mistake of confusing the heat sensation with the cold sensation: Confirming Neumann and others, we found the cold sensation to be more distinct and better localized than the heat sensation.

This certainly does not seem to indicate that the stomach mucosa is devoid of temperature sensations, nor that this sensation is projected from the esophagus, as has been suggested by a number of investigators. However, there is a possibility that some of the water might reach the wall of the lower end of the esophagus by being forced up along the tube. In view of the small amount 
of water necessary to evoke the hot and cold sensations and in view of the short latent period mentioned above, this seems very unlikely.

To make sure that the sensation did not arise as a result of the stimulation of the esophagus, as well as to prove that the sensation is not due to conduction to the skin of the abdomen, or to reflex vascular changes in the skin of the abdomen, a fourth series of experiments was performed. For these experiments a rubber tube I $\mathrm{cm}$. in diameter was used. Inside this large tube we placed two smaller tubes, side by side; one of these was $5 \mathrm{~mm}$. in diameter and the other was $3 \mathrm{~mm}$. The smaller of these two tubes was not pulled clear through the outside tube, but only so far that its lower end was about an inch above the lower end of the outside tube. The $5 \mathrm{~mm}$. tube was pulled through the outside tube so that it extended about $10 \mathrm{~cm}$. below the lower end of it. A small glass tube $5 \mathrm{~mm}$. long was now forced up into this longer tube as far as to the lower end of the outside tube. The lower end of the outside tube was then tied securely about the longest tube at this point. This apparatus was now swallowed far enough down, so that the lower end of the outside tube reached just through the cardiac orifice. The longest tube, the end of which of course was open, therefore extended far down into the stomach. The smallest, whose lower end was within an inch of where the outside tube was tied, that is, within 2 to $3 \mathrm{~cm}$. of the cardia, was now connected with a pressure bottle. It had previously been found that hot water, in passing through a glass tubing and connections of the pressure bottle, lost approximately $8^{\circ} \mathrm{C}$., and that water placed in the bottle at $10^{\circ} \mathrm{C}$. was raised to $12^{\circ} \mathrm{C}$. in passing through. Water at $58^{\circ} \mathrm{C}$. was now placed in the pressure bottle and permitted to flow down through the smallest inside tube. It naturally was forced up again between and around the two inside tubes. By so doing the heat was conducted through the wall of the outside tube and stimulated the heat nerve-endings in the esophagus, so that a definite heat sensation was felt along its whole course, as well as in the mouth and pharynx. The striking feature about this, however, was that, although the walls of the lower end of the 
outside tube naturally became hot before those of the upper, the heat sensation was first felt in the mouth and throat and then gradually traveled down the whole length of the esophagus. At all times, however, the heat sensation was more intense in the throat and in the upper part of the esophagus than in its lower end. While the walls of the esophagus were thus being stimulated, 50 c.c. of water at $50^{\circ}$ to $55^{\circ} \mathrm{C}$. was injected into the stomach through the longest inside tube which opened into the stomach, and after a latent period of about ro seconds, a spreading heat sensation was felt lower down than that resulting from the stimulation of the esophagus. The sensation also seemed better localized than that from the esophagus, so that it was not very difficult to keep the two sensations apart in consciousness. The reason for the better localization might, of course, be due to the fact that more nerve-endings were stimulated in the stomach mucosa, as the water came into direct contact with it, than in the case of the esophagus, where probably the rubber tube did not come into contact with the mucosa at all points.

When water at $10^{\circ} \mathrm{C}$. was placed in the pressure bottle-the outside tube stimulating the esophagus thus being filled by water at $12^{\circ} \mathrm{C}$. and $50 \mathrm{C} . \mathrm{c}$. of water at $12^{\circ} \mathrm{C}$. was injected into the stomach, a distinct, spreading, cold sensation was felt lower than that resulting from the stimulation of the esophagus. There was no difficulty in recognizing two separate and distinct cold sensations, one coming from the esophagus and the other from the stomach.

While the esophagus was being stimulated by water at $12^{\circ} \mathrm{C}$. in this tube system, a water bottle which was filled with water at $12^{\circ} \mathrm{C}$. was placed on the skin of the abdomen. The two cold sensations resulting could easily be kept apart in consciousness. Next, 50 c.c. of water at the same temperature were injected into the stomach, and a third, distinct, spreading, cold sensation was felt in the stomach. This experiment was repeated several times with hot and cold water, as indicated above, and with the same results.

If the temperature sensation felt on introducing hot or cold liquids into the stomach is due to heat conduction through to the 
skin of the abdomen, or to reflex vascular changes in the skin of the abdomen, or is projected from the esophagus, how is it possible that one can distinguish in consciousness the sensations coming from the esophagus, the stomach, and the skin, at the same time, when each is being stimulated by water at the same temperature, as shown above?

To make sure that the sensation was not due to conduction through the abdominal wall to the skin of the abdomen, the bulb of a thermometer was pressed close to the skin over the stomach and covered with absorbent cotton. After it had become stable, 200 c.c. of water at $10^{\circ} \mathrm{C}$. were injected into the stomach and the mercury in the thermometer watched for a period of 5 minutes. This was repeated several times. In each case, after a latent period of 5 minutes, the mercury had fallen only a small fraction of a degree. It is therefore evident that the cold sensation which one feels after a latent period of from 5 to ro seconds, after the injection of 20 c.c. of cold water into the stomach, is not due to conduction through the abdominal wall and stimulation of the nerve-endings in the skin.

Boring has shown that 500 c.c. of water at $0^{\circ} \mathrm{C}$. in the stomach lowers the skin temperature only $0.05^{\circ} \mathrm{C}$. It is therefore out of the question that 25 c.c. of water in the stomach at $25^{\circ}$ to $30^{\circ} \mathrm{C}$. stimulate the temperature end organs in the skin. Moreover, the adequate temperature stimulus in the stomach cavity is less than $5^{\circ}$ above and $10^{\circ}$ below the body temperature.

We agree with Hertz that when one swallows a mouthful of ice water, the most intense cold sensation seems to come from the lower end of the esophagus, and that this is undoubtedly due to the fact that the water accumulates there and is detained for a shorter or longer period before the cardiac orifice opens so that it can flow into the stomach. He further states that the cold sensation disappears after the water has entered the stomach. This we cannot corroborate. We invariably feel a vague, spreading, cold sensation after the water has entered the stomach, which disappears after a few seconds. Even if it were the case that no cold sensation could be felt after the water had passed into the 
stomach, this could not prove that the stomach mucosa is devoid of heat and cold nerve-endings. In the first place, a mouthful of cold water in passing down into the stomach is warmed sufficiently to raise its temperature several degrees. On this account the nerveendings in the mucosa of the stomach are not stimulated as intensely as are those in the esophagus. The empty stomach contains ro to 50 c.c. of fluid at $38^{\circ} \mathrm{C}$. which rapidly mixes with and thus raises the temperature of the swallowed water. It is also well known that stronger impulses, reaching the central nervous system from one part, tend to suppress in consciousness weaker impulses of the same nature, reaching it from some other part, so that it is difficult to separate the two sensations in consciousness.

From our experiments we conclude: (I) The stomach mucosa is endowed with heat and cold nerve-endings. (2) These fibers are, as Head suggests, of the protopathic type; that is, they are not stimulated by slight temperature changes, or if they are, the impulses do not affect consciousness. (3) They are more abundant, or more readily stimulated in the throat and esophagus than in the stomach.

\section{THE SENSATION OF FULNESS AND SATIETY}

All who have studied the origin of the sensations of fulness and satiety seem to agree that they do not originate in the mucosa of the stomach, and our own results agree with this view. The literature is extensively reviewed by Hertz.

Hertz and his co-workers inflated the stomach of two healthy men with air through a tube connected with a manometer. They found that a "sensation of fulness or tightness in the upper part of the abdomen, associated with a desire to eructate, was felt as soon as the intragastric pressure reached respectively I 2 and $14 \mathrm{~mm}$. of mercury in the two cases. The pressure fell after $20^{\circ}$ seconds by approximately $2 \mathrm{~mm}$., owing apparently to relaxation of the tone of the stomach, and simultaneously the sensation of fulness disappeared. Now, on slowly injecting more air, the pressure gradually rose to its original height and the sensation reappeared; it again disappeared after 20 seconds, the pressure simultaneously falling $2 \mathrm{~mm}$., after which it remained constant. Exactly the same 
rise in pressure and the same sensation of fulness, followed by a fall of pressure and a disappearance of the sensation, were produced four times in succession by injecting air, none being allowed to escape in the interval. These observations proved that the tension exerted from within on the circular muscle fibers of the stomach is the cause of the sensation of fulness."

That this is the main source of its origin seems quite clear. There is a possibility that the stretching of the abdominal muscles as well as the pressure on other structures in the abdominal cavity contribute to the sensation of fulness. As a proof that this element has little if any significance in giving rise to the sensation, Hertz related experiments on persons with atonic stomachs. When $6 \mathrm{gm}$. each of sodium bicarbonate and tartaric acid are swallowed separately, I,700 c.c. of carbon dioxide are given off under atmospheric pressure at body temperature. This invariably causes an unpleasant and sometimes painful sensation of fulness in normal individuals, while in persons with atonic stomachs it does not cause any sensation of fulness. Hertz, therefore, concludes that "the sensation of fulness in the stomach is due to tension on its muscular coat, and depends very little and only in extreme cases on the stretching of the abdominal wall."

To these observations of Hertz we can add our negative findings in regard to the gastric mucosa as a contributing factor. Chemical or mechanical stimulation of the mucosa (whether the stomach is in strong or in feeble tonus) never produces a sensation similar to that of fulness. At the same time, it must be noted that mere tension on the muscular coats, that is, intragastric pressure, will not under all conditions give rise to the sensation. The degree of intragastric pressure required to cause a feeling of fulness, according to Hertz, is frequently exceeded at the height of a period of hunger contractions of the empty stomach, when a distended balloon is in the stomach, yet the sensation referred to the epigastrium in these conditions is that of emptiness, not fulness. It is therefore clear that a certain amount of tonus reaction of the stomach must be present before tension or pressure on the walls of the stomach produce the sensation of fulness. 
The sensation of saticty felt after an abundant and palatable meal involves several factors, none of which appear to depend primarily on the nerves in the gastric mucosa. One must have some degree of hunger and appetite before eating, the food must be palatable, and enough food must be consumed to produce moderate distension of the stomach. If either of these factors is lacking, complete satiety is not felt after the meal. One may feel great hunger and consume unpalatable food till the sensation of fulness develops, but satiety does not follow. If the hunger factor is present and the food is to the king's taste, but of insufficient bulk, although more than ample for the needs of nutrition, satiety is also lacking. Hence the sensation complex of satiety involves the element of contrast between the uncomfortable tension of hunger and the sensation of fulness, together with the lingering memories of the taste and smell of the food. Normal motor, secretory, and sensory functions in the stomach are thus a prerequisite rather than a direct factor in the genesis of satiety.

\section{NAUSEA OF GASTRIC ORIGIN}

The sensation complex of nausea and vomiting can be initiated by stimulation of nerves in the gastric mucosa; but many of the sensory elements in nausea involve mechanism outside the stomach. When nausea is induced by the odor, sight, or taste of disgusting substances, or by the memory of such substances we may call it strictly physiological processes. But most cases of nausea are due to pathological processes or disease. Some of these pathological conditions are pressure, partial anemia, or action of specific toxic substance on the medulla; intense and prolonged pains of peripheral origin, toxemias, hyperexcitability, or excessive stimulation of the visceral nerves.

The sensation of nausea is very complex. A certain feeling of bodily weakness and a characteristic pain and distress ("sinking feeling") in the stomach are always" present. With these one may also experience headache, dizziness, chills (from cutaneous vasoconstriction and perspiration), formication, etc. The violent contractions of the pyloric end of the stomach pre- 
ceding and accompanying the act of vomiting may even be felt as painful.

When nausea is produced by excessive or destructive stimulation of the normal gastric mucosa, or by what may be called normal stimulation (water, food, gastric juice, etc.) of a hyperexcitable mucosa, what sensory elements in nausea are due to this stimulation directly? Since strong contractions of the stomach cause a pressure-pain sensation, and excessive stimulation of the mucosa may also cause pain, it is evident that the tension, pressure, and pain referred to the epigastric region in nausea are of peripheral or gastric origin. The peculiar "sinking feeling" or the feeling of "helplessness" is probably in the main also a direct effect of the gastric states. At any rate that feeling is not experienced except in nausea sufficiently intense to approach vomiting. The weakness, dizziness, and headache are probably in the main indirect effects, that is, due to disturbances in the circulation.

Michael Foster considered "nausea allied to hunger." Boring states that some of his subjects (normal men) confused mild nausea with the sensation of hunger. Thus 5 per cent of hydrochloric acid put directly into the stomach by a tube produced gastric pain identical with hunger, except that it was more continuous. One of Boring's men who was subject to spells of indigestion reports "when I feel nauseated [from indigestion] I generally stop eating. I begin with my meals again as soon as I feel hungry, but I cannot always tell whether I am hungry or still nauseated."

We believe this fusion or confusion of nausea with hunger is either pathological or else due to superficial analysis. It is true that the gastric factors in both hunger and nausea are uncomfortable tension and pain; that hunger as well as nausea causes salivation; that in some people hunger as well as nausea includes bodily weakness, headache, etc., but the distinct "sickness" character of the gastric distress in nausea is not even present in any stage of hunger in normal persons. Hunger involves the kinesthetic nerves of the stomach, while nausea is caused by stimulation of nerves in the gastric mucosa. The central effects of hunger and nausea in normal persons are also different. Hunger is compatible with 
and intensifies appetite and desire for food, while nausea and appetite are mutually exclusive. But since hunger is, and nausea may be, caused by stimulation of gastric nerves, it is likely that in persons with hypersensitive vagi, or with unstable central nervous organization, strong hunger may contain elements of nausea, or actually pass into nausea.

Normal persons may experience hunger pangs and something like mild nausea or "sick stomach" at the same time, without confusing the two sensations, provided there is a certain degree of hyperexcitability of the nerves in the gastric mucosa. This was noted by the author on the fifth day of starvation. On that day the sensation of hunger was tinged with a "burning" sensation also referred to the stomach. This sensation was probably caused by pressure or acid stimulation of hyperexcitable nerve-endings in the gastric mucosa. It is known that prolonged starvation leads to such hyperexcitability, and in this case there was additional indications of it in the nausea caused by smoking.

\section{THE GASTRIC ELEMENT OF APPETITE}

Substances like strong alcohol or acid ( 5 to 20 per cent $\mathrm{HCl}$ ), mustard, pepper, etc., introduced by tube directly into the stomach in sufficient quantities, cause varying degrees of pain, accompanied at first with a peculiar feeling of warmth in the stomach. In concentrations sufficient to cause pain, all chemicals evidently injure the mucosa and its nerve-endings, as shown by the development of gastritis. Boring states that the pain induced by 2 to 10 per cent $\mathrm{HCl}$ in the stomach is identical with the pangs of hunger, and surmises that the acid in the stomach causes gastric hunger contractions. This surmise is contrary to all experimental facts, at least on normal men and dogs.

When these and other chemical substances are put into the stomach too dilute to cause ache, pain, or discomfort, their contact with the normal gastric mucosa still affects consciousness, but in a manner the very opposite to that of pain. The sensation produced by this gentle chemical stimulation of the mucosa has a character of its own that resembles if it is not identical with appe- 
tite. We cannot understand how Hertz and Schmidt could have reached the conclusion, on the basis of experiments on man, that the normal mucosa is insensitive to chemical stimuli, including 2 per cent mineral acids $(\mathrm{HCl})$.

Pavlov concludes that "the tactile sensation of the stomach at the moment of entry of food is capable of awakening or increasing appetite." In the experiments cited by Pavlov in support of this conclusion the stimulation of the nerve-endings in the mouth and in the esophagus was not excluded.

The author, working on himself, soon recognized that moderate chemical stimulation of the nerve-endings in the gastric mucosa modified the flow of consciousness, although this modification did not consist in the sensation of hunger. This can readily be experienced by anyone who is sufficiently interested to try, by introducing moderately cold water, beer, wine, weak acids ( 0.5 to 2.0 per cent $\mathrm{HCl}$ ), weak alcohol, or carbonated drinks through a tube into the stomach so as to avoid stimulation of nerve-endings in the mouth and esophagus. The sensation produced by these substances in the stomach is rather transitory, but may persist for several minutes. With the exception of cold water, which is also felt as cold, these various substances give rise to a characteristic sensation which fuses with, or cannot be distinguished from appetite. It is like the sensation of increased appetite experienced by most people at the beginning of a meal, after eating a few morsels of palatable food. The sensation is pleasant, and turns the attention toward food and eating.

We are accustomed to think that the substances named above affect consciousness solely through stimulation of nerve-endings in the mouth. This view is no longer tenable. By introducing these substances through the stomach tube at the height of a gastric hunger contraction, one actually experiences a successive contrast of the sensations of hunger and appetite, as these substances temporarily inhibit the hunger contractions in stimulating the gastric mucosa. From the first it was clear that, when beer or cold or hot water was first introduced into the stomach during a vigorous hunger contraction, the sensation resulting was the exact opposite 
of that caused by the hunger contraction. In place of an unpleasant tense sensation, associated with restlessness, the sensation caused by these different stimuli is one of relief. A pleasant, tingling sensation is felt in the stomach. One feels perfectly at ease, but the thoughts tend to revert to the dinner table. At first, we were not able to say just what this sensation was like, although it was a familiar one. After paying close attention to the sensation experienced at meals just after a few mouthfuls of good food or drink have been swallowed, we became convinced that the two sensations are very much alike, if not identical.

How do we know that the sensation temporarily produced by the above-named. substances in the stomach is directly due to stimulation of nerve-endings in the gastric mucosa?" Since the introduction of these substances in the stomach inhibits the gastric tonus and the gastric hunger contractions, may not the sensation be one of negative character, so to speak, that is, diminution or absence of hunger? We are in position to answer this question definitely in the negative. In the first place, the sudden and spontaneous relaxation of the stomach at the end of a period of gastric hunger contractions is accompanied by a characteristic sense of relief and disappearance of a certain tension or unpleasant mental stress, but this sensation complex has not the positive character that directs attention to food and eating. It is essentially relief from pain. Secondly, putting these substances into a stomach which is quiescent and very greatly relaxed, nevertheless, inaugurates this temporary appetite or appetite-like sensation. Hence we conclude that it is directly induced by stimulation of certain nerve-endings in the gastric mucosa itself. Of course, if the nerveendings in the gastric mucosa are thus stimulated at the time the muscularis is in strong tonus and hunger contractions, the appetitelike sensation is fused with that of relief from the pangs of hunger.

It is significant that normal human gastric juice having full acid strength $(0.45$ to 0.50 per cent free $\mathrm{HCl})$ is capable of inducing this sensation from the stomach. This has been verified repeatedly by introducing 50 c.c. of appetite gastric juice into Mr. V. through the stomach tube. Gastric juice of weaker acidity ( 0.20 per cent) 
does not have this effect. As this full-strength gastric juice is rapidly secreted in the stomach at the beginning of eating, it is probably a factor in the augmentation of appetite by the very act of eating. It may be pointed out that the foregoing facts permit us to see a suggestion of truth in Beaumont's theory that turgescence of the gastric glands is the cause of hunger. In the first place, Beaumont, in common with most physiologists, did not clearly distinguish between hunger and appetite, but used the two terms interchangeably. If we, then, substitute appetite for hunger, we see that with the stomach normal, the appetite sensation may be actually initiated or augmented by gastric juice, not through mechanical pressure of the juice in the ducts, but by acid stimulation of nerve-endings in the gastric mucosa.

It need scarcely be pointed out that when foods or liquids are taken into the mouth and swallowed in the. normal way their main influence on appetite is by way of nerve-endings in the mouth. In fact, this influence is so predominant that only by excluding it are we able clearly to distinguish the gastric factor. The memory factor in appetite is therefore pre-eminently gustatory and olfactory. 


\section{CHAP'TER VIII}

\section{HUNGER AND AGE}

\section{HUNGER IN INFANTS}

That the young and growing individual experiences greater hunger than the adult or aged individual is common knowledge. This is an obvious biological correlation. In addition to the formation of new tissues, the young mammal is usually more active than the adult, and its body surface is larger in proportion to body weight, and hence (if warm-blooded) he loses proportionately more heat. All these factors call for greater amounts of food. There are a number of conditions that may operate to produce this greater hunger in the young. (I) There may be a greater rate of secretion of gastric juice, so that the digestion in the stomach requires less time. This would give a more continuously empty stomach. There may be relative hypermotility of the filled as well as of the empty stomach because of the actual youth of the stomach tissues, because of greater motor innervation from the brain (vagus tonus), or as the result of greater concentration of specific chemical stimuli in the blood correlated with the higher rate of metabolism. (3) There may be greater sensitiveness to afferent impulses on the part of the brain concerned in the hunger sensation. Either factor alone, or all combined, would result in greater frequency and intensity of hunger and appetite.

The first question to be determined is whether the stomach of the young exhibits greater hunger contraction than the stomach of adult and aged individuals of the same species. On the basis of work done on man and dogs this question can now be answered in the affirmative, at least for these two species.

The empty stomach of the newborn infant shows the periods of gastric hunger contractions before the infant has had any experience with food: The hunger periods are more frequent in the infant than in the adult; that is to say, the duration of motor 
quiescence of the empty stomach between the hunger periods is shorter. In the newborn and very young infant the quiescence of the empty stomach lasts from ro to 60 minutes; in the adult usually from I to 3 hours.

This greater frequency of hunger in the child is also shown by the more rapid development of the hunger period after a previous meal. In an adult (after a full meal) gastric hunger contractions do not develop for from 4 to 6 hours. If the individual is lying in bed, the time is even longer. In the normal breast-fed infant the average time of appearance of a hunger period after a full meal is only $2 \frac{1}{2}$ hours.

Pediatricians differ as to the incidence of hunger in infants, because up to the last few years there has been no certain objective criterion for the existence of hunger. This has led to varying views and practices in regard to the frequency with which infants should be nursed, some favoring an interval of 2 hours, some one of $2 \frac{1}{2}$ to 3 hours, and some a 4 -hour interval between nursing. Since the contractions peculiar to the cardiac and fundal ends of the empty stomach are, in normal individuals at least, an objective index of hunger, the balloon method enables us to determine the time of onset of hunger in infants after previous feeding, and the answer to this question may aid in fixing the time that should elapse between the nursings of normal infants.

Our observations were made on thirty normal breast-fed infants, from 24 hours to 4 weeks old. In every case the infant nursed until it was satisfied. Introduction of the balloon into the stomach directly after nursing leads to vomiting of the balloon and some of the food. The balloon is readily retained from 45 minutes to I hour after feeding. Forty-five observations were made on the thirty infants. The average time between nursing and the appearance of the period of hunger contractions was 2 hours and 40 minutes, with a maximum of 3 hours and 30 minutes, and a minimum of 2 hours and 20 minutes.

Our tracings show that when the stomach is full of food the inflated balloon in the fundus reveals practically no contractions. As the stomach gradually empties, feeble tonus contractions appear, 
and increase in rate and intensity until they end in a period of typical hunger contractions from $2 \frac{1}{2}$ to 3 hours after the previous nursing. This change of the tonus contractions of the fundic end of the stomach partly filled with food into the hunger contractions of the empty stomach has already been described. Our results with the balloon method confirm the X-ray observations of Pisek and Le Wald, Czerny, Leo, and others, who contended that the emptying time of a child's stomach is $1 \frac{1}{2}$ to 3 hours. The time required for emptying the stomach depends, of course, on the quantity and quality of the food as well as on the rate of the gastric secretion and the vigor of the gastric digestion peristalsis.

In this connection it may be of interest to note that the mammalian infant, except when under the strict control of a pediatrician feeds, on the whole, as soon as the hunger sensation is strong enough to be uncomfortable, provided food is at hand; and he thrives on this procedure; while the infant whose routine is ordered by the pediatrician nurses every. 2, 3, or 4 hours, irrespective of the onset or intensity of the periods of hunger. Of course, a certain period of rest for the gastric glands may be beneficial.

The infant's stomach shows feeble tonus contractions of the fundal end I hour after nursing. As the stomach discharges its contents these tonus undulations gradually increase in frequency and intensity until by the end of from $2 \frac{1}{2}$ to 3 hours these become transformed into vigorous hunger contractions. The time of onset of hunger contractions after previous feeding varies for each infant. In our present series the minimum is 2 hours and the maximum 3 hours. In the normal individual the presence of vigorous hunger contractions is probably a biologic evidence that the stomach is in proper condition to receive food. If this is the case, the stomach of a normal infant is ready to receive food from 2 to 3 hours after the previous nursing.

\section{HUNGER IN YOUNG DOGS}

The experiments on dogs were made on pups delivered by Caesarian section 8 to 14 days before term, on pups 5 weeks to 5 months of age, on young adults, and on old adults. In all cases 
care was taken to choose only dogs in good condition and perfect health, as far as could be judged by actions and external appearances. Except in the case of the prematurely born pups, the observations were made 24 hours after feeding, in order to assure a completely empty stomach. The animals were given at least one day or more of rest after each experiment, so as to be in a perfectly normal condition when used again. During the taking of the records they were held in the lap, apparently without any appreciable discomfort, for they nearly always slept through a large part of the experiment. Ten different series of records of the hunger movements of the empty stomach were obtained from each dog on ten separate days, the continuous experimental periods ranging from $2 \frac{1}{2}$ to $4 \frac{1}{2}$ hours, respectively.

The results summarized in the accompanying table were computed from the tracings, and the figures in each case represent as nearly as possible the true time of activity and rest of the empty stomach, everything in the records of a doubtful character, or of an abnormal nature caused by disturbing influences, being eliminated.

\section{GASTRIC HUNGER CONTRACTIONS IN DOGS OF DIFFERENT AGES}

\begin{tabular}{|c|c|c|}
\hline Dogs & $\begin{array}{c}\text { Length of } \\
\text { Contraction Period }\end{array}$ & $\begin{array}{l}\text { Length of } \\
\text { Quiescent Period }\end{array}$ \\
\hline 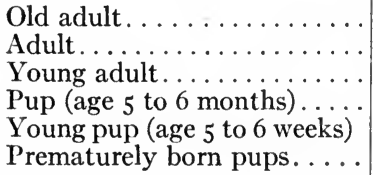 & $\begin{array}{l}30 \text { min. to } 2 \text { hours } \\
1 \frac{2}{3} \text { to } 3 \text { hours } \\
2 \frac{3}{4} \text { to } 3 \frac{3}{4} \text { hours } \\
3 \text { to } 4 \text { hours } \\
4 \frac{1}{2} \text { to } 5 \frac{2}{3} \text { hours } \\
\text { Continuous }\end{array}$ & $\begin{array}{l}I \frac{1}{6} \text { to } 3 \frac{2}{3} \text { hours } \\
I \frac{1}{6} \text { to } 2 \text { hours } \\
\text { I to } 1 \frac{1}{2} \text { hours } \\
5 \text { to } 10 \text { min. } \\
2.5 \text { to } 3.4 \mathrm{~min} \text {. } \\
\text { None }\end{array}$ \\
\hline
\end{tabular}

As regards variation of stomach movements between dogs of different ages, the chief and practically the only constant difference was found in the length of the periods of contraction and the periods of quiescence. In all cases the periods of quiescence are the longest in old dogs, varying from $I_{\frac{1}{6}}$ to $4 \frac{1}{6}$ hours, and rapidly decreasing in length proportionately to age to $2 \frac{1}{2}$ to $3 \frac{4}{10}$ minutes in pups a few days to five or six weeks old. Conversely the periods of 
contraction are the longest in the young dogs-for instance, in the very young pup the recorded periods run from $4^{\frac{1}{2}}$ to $5^{\frac{1}{2}}$ hours-and they rapidly decrease in length proportionately to age -in the old dogs from 30 minutes to 2 hours-thus showing that the stomach's activity is in direct proportion to the age of the animal (Patterson).

The rapidity of the strong hunger contractions during the active periods appears on the whole to be greater in young animals than in old. The tonus of the stomach and also the strength of the contractions in young animals may be slightly higher, but they are subject to great variations. The decrease in the activity of the stomach as the animal approaches senility is probably an explanation, in part at least, of the more chronic gastric disturbances in the aged.

To summarize: in healthy dogs the hunger contractions of the empty stomach decrease with age. This decrease appears to some extent in the tonus and in the rapidity of the hunger contractions, but is particularly marked in the duration of the periods of hunger activity and the intervening periods of quiescence of the stomach. On the whole, the decrease in the gastric hunger activity is proportional to the advance in age. In very young dogs the hunger contractions of the empty stomach are practically continuous.

Two factors are probably involved in this age variation in the gastric hunger contractions, namely, (I) the actual age (reduced metabolism) of the motor tissue in the stomach itself, and (2) a lowering of metabolism in the entire body, which may imply a smaller quantity of chemical stimuli to the gastric hunger mechanism in the circulation. Patterson attempted to determine the relative importance of these two factors by studying the influence of prolonged starvation on the gastric hunger contractions in very young and in very old dogs. If the rate of general body metabolism, that is, starvation metabolism, is the main factor, we should expect that in prolonged starvation the gastric hunger contractions of old dogs would approach the vigor of the normal hunger contractions of young dogs. This is not the case. It is true that starvation causes some increase in the vigor of the stomach tonus and con- 
I24 CONTROL OF HUNGER IN HEALTH AND DISEASE

tractions in the old dogs. Starvation metabolism of the body in general is therefore a factor. But the actual age of the stomach tissues is the most important element. How actual age of the gastric motor mechanism reduces the vigor and duration of the hunger contractions cannot be answered until we know what constitutes the physiological aging of the tissues. 


\section{CHAPTER IX}

\section{HUNGER IN PROLONGED STARVATION}

\section{EXPERIMENTS ON MEN}

In the popular mind prolonged starvation is associated with great pain and distress, despite reliable reports to the contrary from many persons who have undertaken voluntary starvation. Such persons state, almost without exception, that after the first three or four days of starvation, the sensation of hunger is no longer felt, or at least is not excessively painful or uncomfortable.

This brings up some important physiological and biological questions. If hunger ceases entirely, or at least loses its painful urge after a few days of starvation, what makes the starving animal fight for food as long as his strength enables him to move? In man this struggle for food may be due to the desire to live or the fear of death, but can we assume this degree of conscious foresight in the lower animals? Is the reported absence of hunger sensation in man after the first few days of starvation due to failure of the gastric hunger mechanism, or to changes in the brain that prevent the hunger impulses from reaching consciousness? One might naturally expect a decrease in the intensity of the hunger sensation when starvation reaches a point where the brain and stomach are greatly enfeebled by loss of living substance or by too high an acidity of the blood, but this state is not reached in a healthy man by three or four days' deprivation of food. If the hunger mechanism is controlled even in part by the starvation changes in the tissues (stomach, brain) and in the blood, we should expect the hunger sensation to increase in strength up to the point of tissue marked depletion, unless the starvation itself sets up other inhibitory processes.

These questions can be answered only by direct experiments, and these were accordingly undertaken both on man and on the lower animals. The work on man was carried out on the author and one assistant (Mr. L.), in such a way that the gastric hunger 
contractions and the subjective hunger sensation were recorded, day and night, during the entire starvation period. In animals below man we can, of course, record the gastric hunger contractions only, as we have as yet no means of determining the intensity or quality of the sensations caused by these contractions.

During the five days' starvation period the two men continued their usual work during the day, and records of the stomach tonus and hunger contractions were taken at varying intervals. During the night continuous records were taken beginning 8:00 to 9:00 P.M. and ending at 5:00 to 6:00 A.M. Neither the writer nor Mr. L. found much difficulty in sleeping for 6 to 8 hours every night with balloon and tube in the stomach. The room was kept dark, except for a feeble light focused on the drum, so as to enable the assistant to take care of the recording. And the assistant took special care to keep everything as quiet as possible. One assistant took care of the recording from 8:00 A.M. to 2:00 P.M., and a second assistant from 2:00 P.M. to 6:00 A.M. The time from 6:00 to 8:00 A.M. and from 6:00 to 8:00 P.M. was usually spent in walking in the parksin any event, outside the laboratory.

I. THE OBJECTIVE PHENOMENA. THE HUNGER CONTRACTIONS

Before beginning the starvation period, observations on the gastric hunger contractions of the writer were made every second or third day for three weeks. These observations were usually made in the morning after dispensing with breakfast, or else during the middle of the day, thus dispensing with lunch. No observations were made during night or sleep. These records are quite uniform in character, and represent the degree of hunger contractions exhibited by the writer's stomach 6 to ${ }_{5}$ hours after a meal.

We now wish to direct the reader's attention to the following summary of the observations during the starvation period, from June 29 to July 4, I9I4.

$$
\text { A. J. C. }
$$

June 29. No breakfast. First hunger period began gradually at 8:45 A.M., lasting for about 45 minutes; 16 strong contractions in 30 minutes. A second period ended at $\mathrm{I}: 30$, lasting about 30 minutes. There were 22 strong contractions in 30 minutes. 
Last meal. Two slices of toast and a glass of milk at $\mathrm{I} 2: 30$ P.M. Body weight $74 \mathrm{~kg}$.; no observations during the night.

June 30. A.M. II : 50 to I $2: 20$, fairly strong contractions.

I $2: 20$ to $I: 50$, quiescence.

P.M. I : 50 to $2: 30$, I 8 fairly strong contractions.

$2: 30$ to $4: 30$, quiescence.

\section{Interruption}

9:1 5 to 10:00, 22 strong contractions.

Io:०० to II: Io, fairly quiescent.

II: Io to II: 50 , continuous feeble contractions.

II : 50 to I $2: 30,36$ strong contractions.

July I. A.M. I $2: 30$ to $12: 40$, quiescence.

I $2: 40$ to I: 20 , continuous feeble contractions.

$\mathrm{I}: 20$ to $2: 25,45$ strong contractions.

$2: 25$ to $2: 50$, continuous feeble contractions.

$2: 50$ to $3: 30,27$ strong contractions.

$3: 30$ to $4: 00$, quiescence.

$4: 00$ to $4: 30$, continuous feeble contractions.

$4: 30$ to $5: 20,26$ strong contractions.

Interruption

$8: 45$ to $9: 55,43$ strong contractions.

Io: 20 to I $2: 20$, contractions all the time, but feeble.

P.M. I 2:20 to I:०0, I9 strong contractions.

\section{Interruption}

$3: 45$ to $4: 00,20$ fairly strong contractions.

\section{Interruption}

$8: 35$ to $9: 25,37$ strong contractions.

9:25 to II: 20 , continuous, fairly strong contractions.

II $: 20$ to I $2: 25,47$ very strong contractions.

July 2. A.M. I $2: 25$ to I $2: 50$, fairly quiescent.

I $2: 50$ to $2: 35$, continuous, fairly strong contractions (60).

$2: 35$ to $3: 30$, fairly quiescent.

$3: 30$ to $4: 50,70$ strong contractions, ending in tetanus (4 min.).

Interruption

$6: 30$ to $7: 40$, quiescence.

$7: 40$ to $8: 40,33$ strong contractions.

Interruption

IO: 25 to II:I5, 29 strong contractions.

Interruption

P.M. I:०० to $3: 00$, continuous, fairly strong contractions, stronger

toward the end.

Interruption 
July 2. P.M. $8: 45$ to $9: \infty 0,22$ strong contractions.

$9: 00$ to $9: 50$, continuous feeble contractions.

$9: 50$ to IO: 30,27 strong contractions.

IO: 30 to II: 30 , continuous, fairly strong contractions.

1 II $: 30$ to I $2: 30,34$ strong contractions.

July 3. A.M. I $2: 30$ to $2: 25$, continuous, fairly strong contractions.

$2: 25$ to $3: 40,60$ strong contractions, ending in incomplete tetanus ( $5 \mathrm{~min}$.).

$3: 40$ to $4: 05$, continuous feeble contractions.

$4: 05$ to $5: 00,42$ strong contractions.

\section{Interruption}

$8: 30$ to $9: 25,30$ strong (not maximum) contractions.

I $\mathrm{I}: 25$ to $\mathrm{I}: \infty 0$, continuous, fairly strong contractions.

I : 00 to $I: 40,30$ strong contractions.

\section{Interruption}

$4: 45$ to $5: 15$, continuous feeble contractions.

$9: 00$ to $9: 45,38$ strong contractions.

$9: 45$ to I I : 20 , continuous feeble contractions.

I I : 20 to I $2:$ IO, 39 strong contractions.

July 4. A.M. I $2:$ Io to I: Io, continuous feeble contractions.

I : Io to $2: 35,60$ strong contractions.

$2: 35$ to $3: 40$, fairly quiescent.

$3: 40$ to $5: 40,50$ strong contractions.

End of experiment: Body weight, $69.8 \mathrm{~kg}$. Loss of body weight, $4.2 \mathrm{~kg}$.

Confining our attention for the present to the objective phenomena, i.e., the gastric tonus and the hunger contractions, we find the following facts to be apparent:

I. There is no decrease in the gastric tonus and the hunger contractions, but, on the contrary, an increase, especially in the tonus, and in the frequency of the hunger periods. An increase in the intensity of the hunger contractions is also evidenced in the appearance of the incomplete hunger tetanus on the fourth and fifth days of starvation.

2. There was a continuous but probably scanty secretion of gastric juice during the entire hunger period, as the balloon on being withdrawn from the stomach always tasted acid and gave the acid reactions.

The five days' hunger period of Mr. J. H. L. began on July I4 and was concluded on July I9. During July io to I4 daily 
observations were made of the gastric hunger contractions, in order to establish the normal frequency and general character of these contractions in Mr. L. The periods of hunger contractions of Mr. L. under normal conditions of eating differ from those of the writer by usually ending in the incomplete tetanus previously described in Mr. V. This is probably due to the fact that Mr. L. is sixteen years younger than the writer.

Summary of the Observations on Mr. J. H. L.

Control Period

July Io. No breakfast or lunch; I:30 to $4: 25$ P.M., period of observation; $2: 00$ to $2: 40$ P.M., gradually increasing contractions ending in II strong contractions and strong tetanus $\left(3 \frac{1}{2} \mathrm{~min}\right.$.). Feeble rapid contractions began to reappear at 3:15 and gradually increased in amplitude.

July II. No breakfast; 9:00 A.M. to I2:00 P.M., continuous fairly strong contractions. Period of $I 5$ strong contractions; IO: 30 to II :00 A.M. and II: 00 A.M. to I2:00 M., continuous fairly strong contractions (22 contractions).

July I2. No breakfast; 9:35 to I0: IO A.M., 22 contractions of gradually increasing strength in 30 minutes, ending in 3-minute incomplete tetanus.

July I3. No lunch; I2:00 to I2:30 P.M., continuous, fairly strong contractions. A small group (IO) of strong contractions I 2:30 to I 2:45 P.M. A period of strong contractions (22 gradually increasing ending in 3 -minute tetanus) I:50 to $2: 30$ P.M.

July I4. IO:०0 A.M. to I:०0 P.M. No breakfast; IO:०0 to IO:50 A.M., 3 I gradually increasing strong contractions ending in tetanus ( $2 \mathrm{~min}$.); I I:30 A.M. to I $2: 00$ M., I 4 fairly strong contractions, no tetanus; I $2: 30$ to I :०० P.M., 2 I gradually increasing strong contractions ending in a 3-minute tetanus.

\section{Fasting Period}

Last meal, July I4, 2:00 P.M. Body weight, $62.8 \mathrm{~kg}$.

July I5. P.M. $7: 45$ to $8: 25$, I9 fairly strong contractions.

$8: 25$ to $8: 35$, quiescence.

$8: 35$ to $9: 00,28$ fairly strong contractions.

$9: 00$ to $9: 15$, quiescence.

$9: 15$ to $9: 45$, period of 2 I fairly strong contractions.

$9: 45$ to $9: 55$, quiescence.

9:55 to Io: 25 , period of 2 I fairly strong contractions. I0: 25 to II:00, period of 18 fairly strong contractions. II :05 to II:40, period of I 7 fairly strong contractions. 


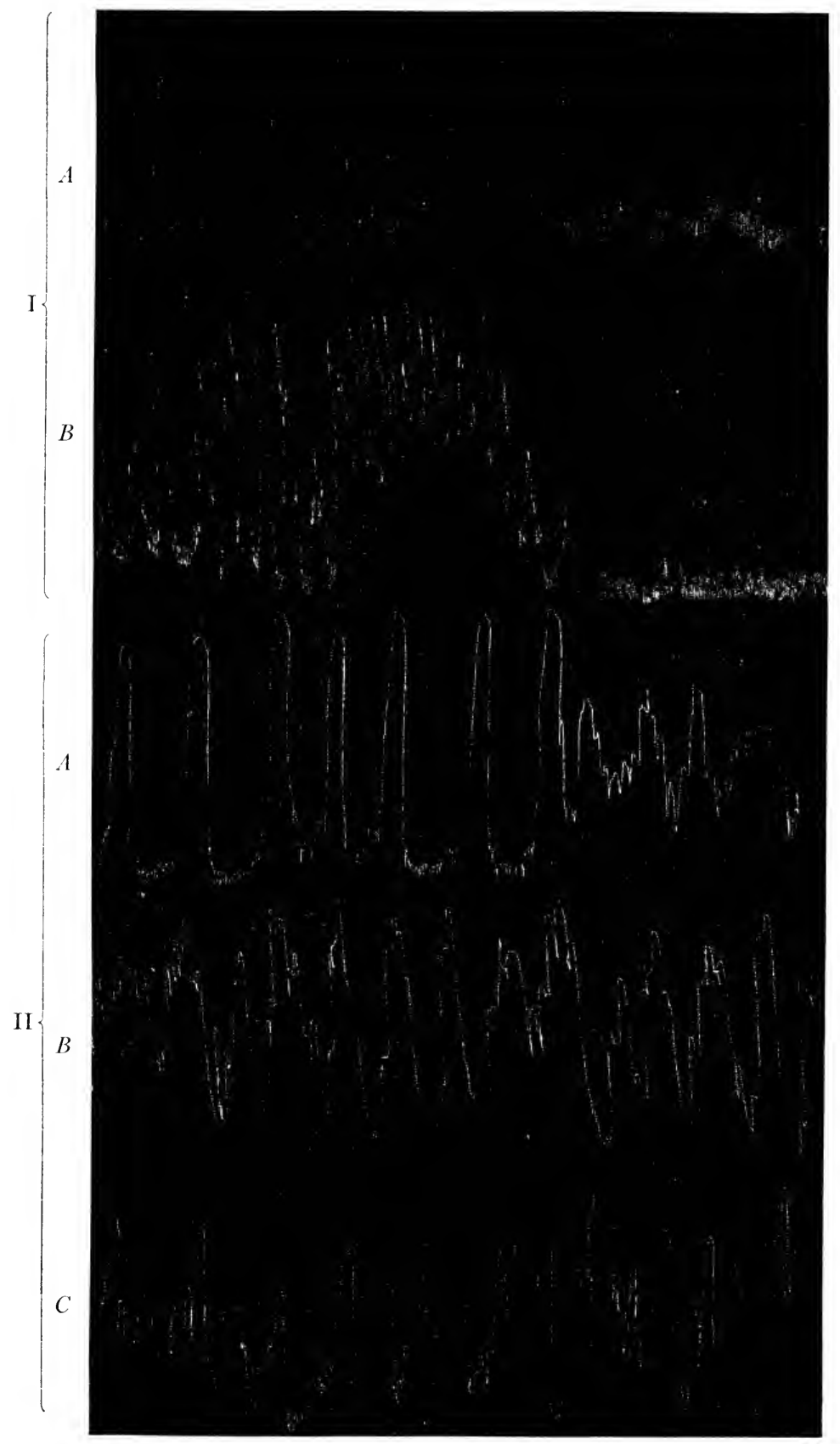

FIG. I5 
FIG. 15.-I. Records of the contractions of the empty stomach of A. J. C. Bromoform manometer. $A$, final ten minutes of a typical period of hunger contractions ten hours after a meal; $B$, final after ten minutes of a typical hunger period after five days' starvation. Note in tracing $B$ the prolonged period of incomplete tetanus at the culmination of the hunger period, and the reappearance of a feeble 20-seconds rhythm immediately following the cessation of the period of strong hunger contractions. Showing the increase in the tonus and the hunger contractions of the empty stomach during prolonged starvation. Four-ninths original size.

II. Typical record (Io minutes) of the hunger contractions of the stomach of dogs. $A$, after 8 days' starvation; $B$, after ten days' starvation; showing a persistence of the gastric hunger contractions during prolonged starvation. $C$, tracing showing end of a gastric hunger period of a dog after 6 days' starvation. 'The vagi and splanchnic nerves sectioned before the starvation period. About one-half original size. 
July I6. A.M. 9:20 to Io: 25 , period of gradually increasing very strong contractions (33), ending in tetanus (2 min.).

Interruption

P.M. I:00 to I:40, period of gradually increasing strong contractions (25), ending in tetanus ( $3 \mathrm{~min}$.).

Interruption

$3: 55$ to $5: 00$, quiescence.

$5: 00$ to $5: 50$, period of fairly strong contractions (I7). No tetanus.

$8: 35$ to $9: 35$, period of very strong contractions (37), ending in 3-minute tetanus.

$9: 35$ to $10: 20$, quiescence.

IO: 20 to II:20, continuous, fairly strong contractions.

II $: 20$ to $I I: 30$, fairly quiescent.

II: 30 to I $2: 00$, period of fairly strong contractions (II). No tetanus.

July I7. A.M. I $2: 00$ to I $2: 40$, continuous feeble contractions.

I $2: 40$ to $I: 30$, quiescence.

I:30 to $2: 00$, continuous feeble contractions.

$2: 00$ to $3: 00$, quiescence.

$3: 00$ to $5: 30$, continuous feeble to fairly strong contractions.

\section{Interruption}

9:30 to I0: 10 , period of very strong, gradually increasing contractions (36), ending in tetanus of $2 \frac{1}{2}$ minutes.

\section{Interruption}

P.M. I2:I5 to $3: 30$, practically continuous, feeble to moderately strong and strong contractions.

\section{Interruption}

$8: 40$ to 10:00, continuous feeble to moderately strong contractions.

I0:00 to 10:40, period of very strong, gradually increasing contractions (20), ending in tetanus ( $2 \mathrm{~min}$.). Io: 40 to Ir:OO, quiescence.

II :00 to II: 50 , period of moderately strong contractions (23). No tetanus.

July I8. A.M. II:50 to 2:30, mainly quiescent (occasional feeble contractions).

$2: 30$ to $3: 30$, period of very strong, gradually increasing contractions (34). No tetanus.

$3: 30$ to $4: 00$, fairly quiescent (occasional feeble contractions). 
July I 8. A.M. 4:00 to 5:00, period of strong, gradually increasing contractions (24), ending in tetanus ( $\left.2 \frac{1}{2} \mathrm{~min}.\right)$.

$5: 00$ to $5: 45$, continuous feeble contractions.

\section{Interruption}

9:20 to I0: I0, period of fairly strong contractions (35). No tetanus.

Io: Io to II: I5, fairly quiescent.

II:I5 to I2:00, period of strong contractions (22). No tetanus.

\section{Interruption}

P.M. 2:30 to $3: 50$, continuous, very feeble contractions.

$3: 50$ to $4: 05$, period of to fairly strong contractions.

$4: 05$ to $4: 15$, quiescence.

$4: 15$ to $4: 40$, period of 13 strong contractions.

$4: 40$ to $4: 50$, quiescence.

$4: 50$ to $5: 30$, period of very strong contractions (22), ending in tetanus.

\section{Interruption}

$8: 35$ to $9: 20,32$ strong contractions, practically continuous. Strong tonus.

9:20 to 10:00, continuous feeble contractions.

I0:00 to I0: 50 , I 5 strong contractions (long drawn out). One tetanus period.

IO: 50 to II:30, period of strong contractions (22), ending in very strong tetanus ( $3 \mathrm{~min}$.).

bI : 30 to I 2:40, fairly quiescent, but a few fairly strong contractions.

July 19. A.M. I $2: 40$ to I:25, period of very strong contractions (37). No tetanus.

I: 25 to $I: 45$, quiescence.

I: 45 to $2: 15$, period of fairly strong contractions (II). No tetanus.

$2: 15$ to $2: 30$, quiescence.

$2: 30$ to $3: 10$, period of very strong contractions (22), ending in a 3 -minute tetanus.

3 : Io to $4: 00$, continuous very feeble contractions.

$4: 15$ to $6: 00$, continuous strong contractions (36), ending in tetanus (3 min.). (Long and irregular pauses evidently due to psychic inhibition, as Mr. L. was very restless.)

End of experiment: Body weight, $59 \mathrm{~kg}$. Loss of body weight, $3.8 \mathrm{~kg}$.

It is clear that the results on Mr. L. practically duplicate those on the writer. (I) There is no decrease, but, on the contrary, an 
increase in the gastric tonus and in the hunger contractions. (2) The stomach showed an acid reaction all the time during the hunger period, evidently due to a continuous but scanty secretion of gastric juice.

\section{THE SUBJECTIVE PHENOMENA}

I. General condition.-Mr. L., as well as the writer, continued in good health and in fairly good spirit throughout the starvation period. On the fourth and fifth days both men felt somewhat weak. Some mental depression was also experienced, especially on the fifth day, by Mr. L., who complained of feeling dizzy on getting on his feet after lying down. An hour's lecturing seemed quite an effort on the fourth starvation day, and on the fifth day both men felt distinctly better when lying down than when sitting or standing. Both slept fairly well during the four nights of the starvation period, despite the persistent hunger contractions and the unusual surroundings of the research laboratory as a sleepingroom. The secretion of urine was diminished, although water was taken whenever desired. In some cases a glass of water was taken to diminish the hunger pangs. The writer did not enjoy a cigar after the second day; in fact, smoking tended to produce nausea. This is an indication of increased excitability of the nerves of the gastero-intestinal canal.

2. The sensations of hunger and appetite.-During the period of control observations both subjects trained themselves in judging the relative intensity of the individual hunger pangs, and in this both of them attained a fair degree of efficiency. They can invariably tell the onset of a hunger period before the contractions have reached a sufficient intensity to be recognized as individual hunger pangs. This initiation of the hunger period consists in a gradually increasing tonus and feeble and more or less rhythmical contractions, and this is felt as a continuous mild hunger or a moderately steady and somewhat uncomfortable tension in the epigastric region. This sensation is not dependent on or influenced by the distended balloon in the stomach cavity. In this way one can usually manage to record practically every hunger period during the day, simply by starting observations as soon as one feels the 
very onset of the hunger period. The periods of strongest hunger contractions, or the hunger tetanus, are also felt as continuous and intense hunger. And there is a characteristic relief from the diminished tension within a minute or so after the period of hunger contractions is at an end.

During the first two or three days the hunger sensations seemed both to Mr. L. and the writer somewhat more severe than any hunger experienced during the control period, in fact, more severe than seemed warranted from the degree of intensity of the gastric contractions. To be sure, the hunger contractions of the stomach were usually somewhat stronger than, and in every case at least as strong as, during the control period, but the hunger sensations seemed even stronger proportionately. During the fourth and the fifth hunger days, on the other hand, the hunger sensation seemed somewhat weaker than one could have predicted from the intensity of the hunger contractions. In fact, the sensation did not even seem to be as keen as that produced by a period of strong hunger contractions 6 to ro hours after the previous meal. The reader will recall that the gastric hunger contractions on the last two days were' of normal or greater than normal intensity.

The sensation of hunger was almost continuous after the first day of starvation. That is to say, the hunger sensation referred to the epigastrium did not wholly disappear during the intervals between the vigorous gastric contractions. This feeble but continuous hunger sensation is evidently caused by the increased gastric tonus and more or less continuous but feeble rhythmical contractions that represent the periods of quiescence of the empty stomach during prolonged starvation. On the fifth day of starvation the continuous hunger sensation seemed to be tinged with a peculiar "burning" sensation, also referred to the stomach, the fusion resembling somewhat the feeling of "sick stomach" with its attendant central depression. This "burning" sensation was probably caused by acid stimulation of hyperexcitable nerve-endings in the gastric mucosa.

The appetite during the starvation period ran practically parallel with the sensation of hunger. It was distinctly increased 
during the first two or three days, and diminished on the fourth and fifth days. In fact, the depression of appetite on these two days seemed distinctly greater than the depression of the hunger sensation. Instead of an eagerness for food, there was almost an indifference toward food, despite the persistent hunger call of the empty stomach. This was particularly true of Mr. L. He stated several times on the fourth and fifth days that the sight of food led, not to a feeling of eagerness for eating, but to a feeling partaking of the nature of revulsion or nausea. This was not experienced by the writer. Food looked good to him throughout the starvation period, but he found it much easier to dismiss thoughts of food and eating from his mind toward the end than at the beginning of the period.

The reasons for this seeming discrepancy in the parallel between the intensity of gastric hunger contractions and the intensity of the subjective hunger sensation during the five days' hunger period can only be conjectured, at present. We are inclined to believe with Stöhr that the weakening of the hunger and the appetite sensations toward the end of the period is due to a depression of the central nervous system. This central depression, however caused, was clearly in evidence both in Mr. L. and the writer. Afferent impulses from the viscera, differing from the normal quantitatively, probably also play a part in the situation.

More prolonged starvation in man appears to lead at times to a heightened or abnormal cerebral activity, as shown by the feeling of exaltation, visual and auditory hallucinations, etc. These phenomena are probably determined quite as much by the type of emotional processes of the individual as by the effects of starvation, since they are reported more frequently by religious ascetics, than by worldly minded men starving for purposes of science or health. The hallucinations may be due to depression of certain cerebral centers, and hence similar to dreaming rather than to actual increase in cerebral excitability. In any case, these phenomena are probably due to starvation changes in the blood and the brain tissues; rather than to the gastric hunger mechanism. 
3. After-effects of the starving period.-Both in Mr. L. and the writer practically all of the mental depression and some of the feeling of weakness disappeared during the partaking of the first meal after the fasting period. This central depression is therefore essentially a reflex condition depending probably on afferent impulses from the digestive tract, rather than a result of lack of nutrient material in the blood. But complete recovery from the bodily weakness did not take place until the second or third day after breaking the fast.

From the second day on both men felt unusually well, distinctly better, in fact, than before the hunger period, although both men are normally in good health and vigor and not hampered by excessive fat. The writer felt as if he had had a month's vacation in the mountains. The mind was unusually clear and a larger amount of mental and physical work was accomplished without fatigue. In the writer's own case, the five days' starvation period increased the vigor of the gastric hunger contractions to that of a young man of twenty or twenty-five, and the empty stomach retained this increased vigor for at least three weeks after the hunger period, when observations were discontinued owing to absence from the University. This improvement or rejuvenation of the stomach is not a matter of subjective feeling of opinion, but a matter of objective record on the tracings. Neither Mr. L. nor the writer can be considered as ordinarily eating to excess, although the daily intake of protein and calories is greater than the minimum requirement advocated by Chittenden. The cause of the improvement was not loss of excessive adipose tissue.

Mr. L. states that the augmentation of hunger and appetite persisted for at least two or three weeks after the end of the starvation period.

We are familiar with but not particularly impressed by the arguments of enthusiasts who advocate starvation as a panacea for various human ills. But this personal experience leads us to suspect that there is more value in some of these measures than is ordinarily considered. Civilized man has traveled far from the conditions of life among wild animals and primitive man, with 
whom periods of starvation are not uncommon. Occasional periods of starvation, say once or twice a year, in the case of healthy adult persons may not only add to the joy of living but also to the length of life. There is some evidence resulting from experiments with animals that periods of starvation may accelerate growth and improve the general body metabolism (Deland, McCollum, Howe, Morgulis).

4. Discomforts of starvation.-During the entire starvation period the hunger sensation was strong enough to cause some discomfort, but not to a degree that could be called marked pain or suffering. This discomfort was at no time sufficient to interfere seriously with work. And since practically all observers agree that the hunger discomfort is greatest during the first few days of starvation, it seems probable that our five days of starvation gave us a taste of the maximum discomfort that would be experienced in more protracted fasts. Accounts of acute sufferings from mere starvation (water being at all times available) must therefore be wholly imaginary, or the result of fear and panic. Voluntary starvation is in no sense a heroic act, and citation of hunger experiments on animals in the interest of science as instances of cruelty to animals is without foundation.

\section{RESULTS ON NORMAL DOGS}

No attempt was made to take continuous records of the motor activity of the empty stomach in the starving dogs. Observations were made for periods of 2 to 6 hours each day, beginning the second or third day of starvation. The result on the five normal dogs can be seen at a glance from the following summary:

\section{Dog I.--Young Vigorous Female}

Starvation Day:

2 d day. IO:25 A.M. to I2:00 M., continuous vigorous contractions; type II and III.

3d day. 2:00 to 4:00 P.M., continuous vigorous contractions, type II mainly.

4th day. IO: 25 A.M. to I2:35 P.M., continuous contractions, type II mainly. 
5th day. I:30 to 3:30 P.M., continuous contractions, type II mainly. 6th day. Io:55 A.M. to I:०0 P.M., continuous contractions, type III mainly.

7th day. 9:00 A.M. to I 2:00 M., continuous contractions, type III mainly. 8th day. 9:35 to II:35 A.M., continuous contractions, type II mainly. 9th day. IO:35 A.M. to I2:35 P.M. continuous contractions, type III mainly.

Ioth day. 9:35 A.M. to $3: 35$ P.M., very vigorous contractions, types II and III only. Tonus on the average, $\mathrm{I} 2 \mathrm{~cm}$. bromoform.

\section{End of experiment}

Dog II.-Young Vigorous Female

3 d day. Io:00 A.M. to 3:00 P.M., strong tonus and continuous contractions of type III.

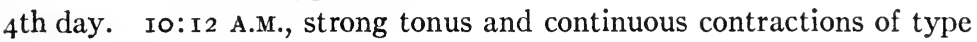
III.

5th day. I:00 to 3:00 P.M., strong tonus and continuous contractions. 6th day. 9:00 A.M. to I 2:00 M., strong and continuous contractions. Tonus about $10 \mathrm{~cm}$. bromoform.

\section{End of experiment}

Dog III.-Old Female

2d day. Io:00 A.M. to I 2:00 M., feeble contractions, type I.

3 d day. 9:00 to I I :०0 A.M., a few irregular contractions, type I; stomach hypotonic.

4th day. I: 00 to 3:00 P.M., a few irregular contractions, type I; stomach hypotonic.

5th day. 8:00 to II:०0 A.M., practically no contractions; stomach hypotonic.

6th day. I:00 to 4:00 P.M., practically no contractions; stomach hypotonic.

\section{End of experiment}

Dog IV.-Young Vigorous Female

3 d day. 9:30 to II:30 A.M., strong tonus, continuous contractions, types II and III.

$4^{\text {th }}$ day. I:00 to 3:00 P.M., fairly strong tonus, types I and II contractions.

5th day. 8:00 to II :00 A.M., strong tonus, types II and III contractions. 7th day. 9:40 A.M. to I2:00 M., fairly strong tonus, type III contractions.

8th day. I:00 to 3:00 P.M., very strong tonus, type III contractions. 
9th day. 8:00 to I0:00 A.M., feeble tonus, feeble contractions, type II. Ioth day. 8:00 to II:०0 A.M., fairly strong tonus; fairly strong type II contractions.

\section{End of experiment}

Dog. V.-Six-Months-Old Female

2 d day. Fast 30 hours; type II contractions; tonus 3 to $4 \mathrm{~cm}$. Chloros form.

3d day. Type II contractions; tonus 3 to $4 \frac{1}{2} \mathrm{~cm}$.

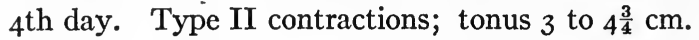

5th day. Type II contråctions; tonus 3 to $4 \frac{1}{2} \mathrm{~cm}$.

6th day. Types II and III contractions; tonus 3 to $4 \frac{3}{4} \mathrm{~cm}$.

7 th day. Types II and III contractions; tonus 3 to $4 \frac{1}{2} \mathrm{~cm}$.

8th day. Types II and III contractions; tonus 3 to $5 \frac{1}{4} \mathrm{~cm}$.

9th day. Types II and III contractions; tonus 3 to $6 \frac{1}{4} \mathrm{~cm}$.

Ioth day. Type III contractions; tonus 3 to $7 \mathrm{~cm}$.

I I th day. Type III contractions; tonus 3 to $6 \frac{1}{2} \mathrm{~cm}$.

I 2 th day. Type III contractions; tonus 3 to $6 \frac{1}{2} \mathrm{~cm}$.

I3th day. Type III contractions; tonus 3 to $6 \frac{1}{4} \mathrm{~cm}$.

I4th day. Type II and prolonged type III contractions; tonus 3 to $7 \mathrm{~cm}$.

I 5 th day. Type III contractions; tonus 3 to $6 \frac{1}{2} \mathrm{~cm}$.

I6th day. Six hours before death from starvation; type II and prolonged type III contractions; tonus 3 to $9 \frac{1}{4} \mathrm{~cm}$.

Dogs I, II, and IV exhibited either normal or greater than normal hunger contractions of the empty stomach during the entire starvation period. The increased tonus of the stomach was particularly marked. In consequence of this increased tonus the types of hunger contractions were usually those previously. described as II and III, that is, practically incomplete tetanus. Judging from observations on man, the dogs probably felt continuous and intense hunger during these contractions.

The only old dog in this series, No. III, had shown rather feeble and irregular gastric hunger contractions before the starvation period. The reason for this was not apparent. The dog was in good condition and would eat greedily, even when the empty stomach was practically quiescent and distinctly hypotonic. This dog showed virtually no gastric hunger contractions after the third day of starvation, and the stomach appeared distinctly hypotonic. The dog was eager for food, however. 
The stomach of the dogs showed acid reaction throughout the hunger period, just as was the case with the stomachs of the starving men.

It will thus be seen that the empty stomach of men and dogs exhibits ordinarily either normal or greater than normal hunger contractions during starvation periods of from 5 to 15 days.

The cause of this increased hunger activity of the stomach may be: (I) an increase in the tonus innervation via the vagi; (2) changes in the blood in consequence of starvation; (3) starvation metabolism of the motor tissues of the stomach itself.

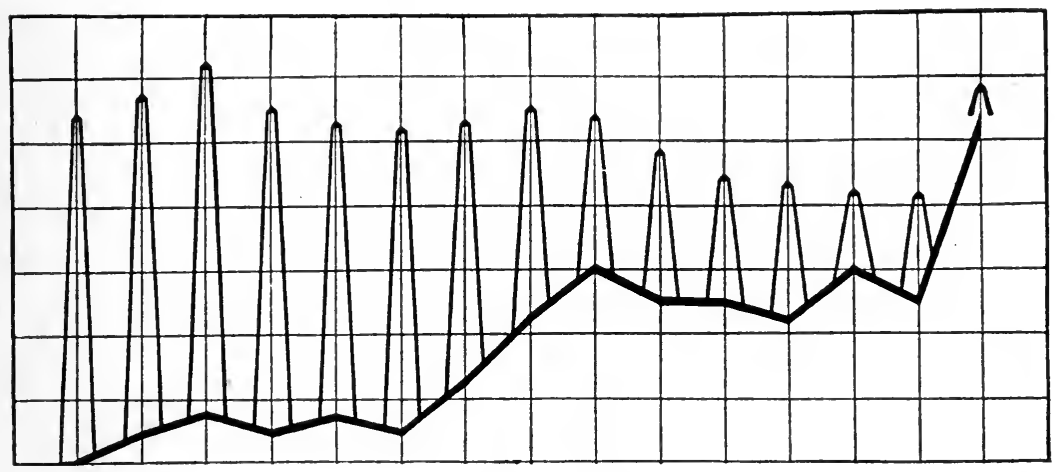

FIG. I6.-Diagrammatic representation of the amplitude of a gastric hunger contraction on the base of the rising tonus as constructed from the daily tracings during starvation of a young dog. Each of the above squares represents I sq. $\mathrm{cm}$. The erect pyramids indicate the amplitudes of the hunger contractions in centimeters arranged on the rising tonus as a base line. Spaces left to right indicate number of days of starvation; spaces from bottom to top daily increase in the gastric tonus in centimeters. Note the rapid increase in gastric tonus and decline in amplitude of contractions on the $\mathrm{I}_{5}$ th day as produced by the prolonged type III hunger contractions (Patterson).

\section{EXPERIMENTS ON DOGS WITH THE STOMACH ISOLATED FROM THE CENTRAL NERVOUS SYSTEM}

In order to decide between these three possibilities starvation tests were made on two dogs with complete isolation of the stomach from the central nervous system by section of the vagi and the splanchnic nerves and on one dog with the vagi nerves severed, 
the splanchnic nerve being left intact. The following is a brief summary of the results:

Dog VI.-Young Female. Section of the Vagi and Splanchnic Nerves Two Weeks before Starvation Period

\section{Starvation Day:}

4th day. Io:०0 A.M. to I:०0 P.M., one group, type I contractions (I5), fairly strong.

5th day. 2:00 to 5:00 P.M., type I contractions, fairly strong but with long intervening pauses.

6th day. I0:00 A.M to I2:00 M., 30 strong (type I) contractions ending in tetanus (Fig. $5^{A}$ ).

7th day. I: 00 to $4: 30$ P.M., continuous contractions of type III; tonus about io $\mathrm{cm}$. bromoform.

\section{End of experiment}

Dog VII.-Adult Fairly Vigorous Female. Vagi and Splanchnic Nerves Cut 5th day. 9:00 to Ir:०0 A.M., 9 very vigorous contractions and tetanus periods.

6th day. I:०0 to 3:०0 P.M., fairly strong contractions of type I; long pauses between contractions.

7th day. I:00 to 5:00 P.M., stomach hypotonic; no contractions.

End of experiment

Dog VIII.-Young, Fairly Vigorous Female. Vagi Nerves Sectioned 3d day. 9:00 to Ir:०0 A.M., fairly strong type I contractions.

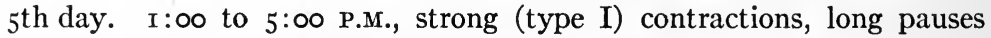
between contractions.

6th day. 9:00 to II:०0 A.M., stomach hypotonic, practically no contractions.

7th day. I:०0 to 4:00 P.M., fairly strong type I contractions, prolonged and partly tetanic; long pauses between contractions.

End of experiment

The gastric hunger contractions of Dog IV were absent on the seventh day of starvation, but Dogs V and VII showed either normal or greater than normal hunger contractions throughout the starvation period. Since the increase in the hunger contractions appeared to be just as marked in the dogs with the stomach isolated from the central nervous system as in normal dogs, it follows that the cause of this increase is not an augmentation of the vagus tonus. 
These starvation periods were not of sufficient length to cause very marked asthenia either in the men or in the dogs (except Dog V). They do show that the hunger tonus and contractions persist with normal or greater than normal vigor during shorter starvation periods. Cases of gastric hypotonus and decreased gastric motility cannot therefore be due to starvation as such, but to some special and exceptional factors. The depression may be due (I) to a primary asthenia of the stomach; (2) to central inhibition via the splanchnic nerves (pains, depressions, etc.); (3) to acid inhibition from the stomach mucosa owing to copious secretion of gastric juice. This question requires further investigation.

\section{EXPERIMENTS ON DOGS WITH PANCREATIC DIABETES}

It is well known that men and other animals afflicted with diabetes lose weight rapidly, despite greater appetite and increased food consumption. In the last stage of diabetes in dogs the animal shows greater emaciation than a normal dog at death from lack of food. Dr. Luckhardt studied the gastric tonus and hunger contractions in two diabetic dogs, and found them persisting and even augmented to within a few hours of death, when the animal is too emaciated and feeble to stand, or to masticate and swallow food. It is true that death from diabetes involves factors that are not present in the normal animal dying from starvation, but dogs dying from pancreatic deficiency have all the appearances of an animal in the most extreme state of emaciation. Nevertheless, there is no gastric atony, and the animal gives evidence of hunger and appetite in his avidity for food to within a few hours of death.

\section{EXPERIMENTS ON RABBITS}

Rabbits succumb to starvation much more rapidly than does man or the dog. Rogers found no indication of depression or rest in the continuous gastric hunger contractions of the starving rabbit until within a few hours of death. Then they became weaker, of shorter duration, and may alternate with periods of quiescence. Shortly before death from starvation the rabbit's stomach may go into periods of strong tetanus, or spasms lasting even for two 
to three minutes. Similar periods of gastric tetanus were observed by Patterson in starving dogs shortly before death. In a normal animal these tetanic contractions of the stomach would give rise to intense hunger pangs, but we cannot know what sensation, if any, they produce in the animal in extreme starvation since we have no data concerning this condition in man. In fact, we do

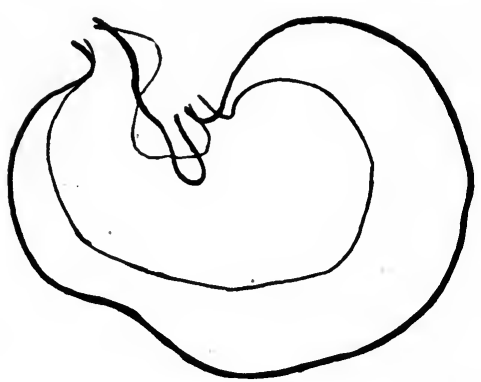

FIG. I7.-Superimposed life-size tracings of the stomachs of two adult rabbits; the larger represents the stomach of a rabbit killed after eating; the smaller the stomach of a rabbit which died of starvation, but had been permitted to eat its own feces (Rogers). not even know the motor condition of man's stomach near death from starvation.

\section{DISCUSSION OF RESULTS}

So far as we are aware, this is the first time that actual records have been taken of the gastric hunger contractions in man during prolonged starvation; and also the first time that the physiologists themselves have done the starving. But we have many accounts of the subjective feeling of hunger and appetite in man during long fasts.

A comparison of these accounts with our own results is rendered difficult by the confusion between the sensations of hunger and of appetite that unfortunately obtains in physiological and medical literature. Leaving out the cases complicated by water starvation, there appears to be a general agreement that the sensations of hunger and appetite increase during the first few ( 2 or 3 ) days of starvation, and then decrease even to complete abolition. Succi reverted to drugs to deaden the hunger pangs only during the first two days of his 30-day fast. Viterbi, who starved himself to death voluntarily and kept a daily record of his feelings, noted complete absence of hunger after the fifth day. Cetti and Breihaupt did not experience any discomfort from hunger after the first few days, but it is not clear that hunger and appetite were altogether lacking. The young man starving for 5 days under the observation of Johansson, Landergren, Sonden and 
Tigerstedt complained of weakness, dizziness, and cold, but did not feel particularly hungry at the end of the fast. Carrington cites many cases of men and women in prolonged starvation where hunger sensation subsided or disappeared after the third day. All these persons cited by the last author were, however, suffering from this, that, or the other ailment, and some of them were lying in bed during the entire starvation period. These remarks apply also to various popular accounts of cases of prolonged fasting to cure digestive or nervous disorders. All cases of compulsory starvation (persons shipwrecked, explorers and hunters lost, or cut off from supplies, etc.) are usually complicated by lack of water, by the effects of exposure, and by fear, panic, etc., so that the state of the actual hunger sensation cannot be determined.

In the case of the $3 \mathrm{I}$-day fast recently reported by Benedict, the subject, a man forty years old, having had previous training in long fasts, insisted that he felt no hunger at any time during the long starvation. Langfeld thinks that this might have been due to strong auto-suggestion, the subject being a vegetarian and a firm believer in fasting as a cure-all. If Benedict's man told the truth, there must have been some abnormality in his hunger mechanism, or else he was able to shunt the visceral hunger impulses out of consciousness by hypnosis. Polimanti states that hunger can be induced by suggestion or hypnosis. We doubt the truth of this statement, at least as regards the real gastric hunger; but if it is true it is probable that hypnosis may also inhibit the hunger.

All these accounts are based on the statements of persons who were in no sense trained observers. In view of that fact, it is probable that the consensus of opinion that hunger disappears after the third day of starvation means no more than that after the third day the hunger sensation is not so persistent or painful as to dominate consciousness.

There was, certainly, some decrease in the hunger and especially in the appetite sensation of the writer, and of Mr. L., on the fourth and fifth days. But it was a decrease, not an absence of hunger sensation. It has already been pointed out that the decrease in intensity of the hunger sensation was not due to a decrease in 
the intensity of the gastric hunger contractions, but to depression of the central nervous system, or some disturbance in the complex of impulses from proprioceptors. We do not deny the possibility that the sensation of hunger may actually disappear in some persons after two or three days of fasting, but this is not due to starvation as such, but to special conditions, such as primary asthenia of the stomach, great cerebral depression, inhibition via the splanchnic nerves through pain or other factors causing hyperactivity of the sympathetic system, or to copious and continuous secretion of gastric juice causing acid inhibition. In view of our results on experimental animals it seems probable that during periods of prolonged starvation the sensation of hunger will prove most persistent in young and vigorous persons.

Boldyreff states that the periodic contractions of the empty stomach in dogs become feeble and irregular during prolonged fasting and cease entirely after a fast of 3 to 4 days. After that time there is a copious and continuous secretion of gastric juice. If this spontaneous secretion of gastric juice is sufficiently rapid, there will, of course, be an acid inhibition of the hunger contractions.

Only one of our dogs (No. III) corroborates the results of Boldyreff. Dog III showed practically no hunger contractions after the third day. The reader will recall that this was the oldest dog in the series, and that he had shown relatively weak and irregular hunger contractions during the control period before starvation. This fact probably indicates an asthenic condition of the stomach, in addition to the certain factor of age.

Boldyreff's dogs had duodenal, biliary, and pancreatic fistulae, in addition to the fistula of the stomach. As these animals were thus subjected to greater disturbance of digestion and metabolism than were the dogs used in our starvation tests, it seems probable that the dogs used by Boldyreff were subnormal in respect to some asthenia of the digestive tract. This may be a factor in the early disappearance of gastric hunger contractions during the prolonged starvation. Multiplication of fistulae also increases the chances for reflex inhibition of the stomach from adhesions, pain, etc. While the difference in Boldyreff's results and our own may 
be due mainly to the difference in the condition of the dogs at the beginning of the hunger tests, it may also be noted that Boldyreff's method of registering the gastric contractions was not delicate enough to show the weaker contractions and the variations in tonus. The strong continuous tonus and rapid contractions (type III) which we have designated the "hunger tetanus" would probably not have been recorded on Boldyreff's tracings. It was precisely this hunger tetanus which was mostly in evidence in our normal starving dogs.

Boldyreff found that during the first three days of starvation there are periods of apparently spontaneous secretion of gastric juice, and that during this secretion the gastric contractions ceased. After the third day the gastric secretion became continuous. We did not subject our dogs to the additional inconvenience of accurate determination of the rate of gastric secretion, but incidental observations in other lines of work on cats with the stomach pouch of Pavlov have convinced us that there may be considerable fluctuation in the rate of the secretion of the empty stomach. The secretion of gastric juice must be relatively rapid, however, in order to maintain complete inhibition of the tonus and contractions of the healthy and vigorous stomach through acid stimulation of local and long reflexes. The stomach of the writer, of Mr. L., and of all the dogs was acid throughout the starvation period, which indicates a more or less continuous secretion of gastric juice, even during the first three days of starvation. But the quantity or strength of hydrochloric acid in the stomach at any one time was not sufficient to produce the acid inhibition either in man, dog, or rabbit.

That starvation in man will ultimately lead to marked weakening (and eventually absence) of the sensation of hunger owing to the depression of the central nervous system and asthenia of the gastric motor mechanism is self-evident, but in young and vigorous animals this depression is absent until the skeletal neuromuscular asthenia is very marked. That prolonged starvation in the case of healthy individuals should completely abolish the sensation of hunger and appetite while the organism is still in fair state of 
integrity is inherently improbable. When it does occur, it is probably due to pathological complications. Starvation increases the desire for food (that is, hunger and appetite) in wild animals, at least up to the point where the asthenia reaches a degree that renders locomotion impossible. This is shown by the increased boldness and disregard of danger on the part of the starving animal (herbivorous as well as carnivorous) in his search for food. 


\section{CHAPTER $\mathrm{X}$}

\section{THE NERVOUS CONTROL OF THE HUNGER MECHANISM}

The question of the nervous control of the gastric hunger mechanism embraces several important physiological problems, none of which are as yet completely solved.

I. On the motor side we have the possibility of actual initiation of the gastric hunger contractions through the motor fibers in the vagi nerves by impulses from cerebral as well as lower centers acting on the motor nuclei of the vagi in the medulla. Even if contractions are not actually caused in this manner, it can be shown that they are in part dependent on a "tonus" influence exerted on the stomach by the vagi nerves. Hence the control of the vagus tonus becomes a question of paramount importance in the physiology and pathology of hunger.

2. On the afferent or sensory side we must determine the central paths of the afferent gastric nerves in order to elucidate the genesis of the conscious hunger sensation as well as the conscious and subconscious reflexes evoked by these afferent impulses. This raises the question of the sensory hunger center in the cerebrum.

3. We have also to deal with the very important reflex control of the gastric hunger mechanism as well as of the nervous foci in the medulla, mid-brain, and cerebrum concerned in the conduction of sensory and motor hunger impulses.

4. And, lastly, we must consider the automatic or reflex elements in the gastric hunger mechanism itself, independent of all central nervous system control. An understanding of these several factors is of particular importance for the interpretation and the control of the changes in hunger and appetite that we meet in disease.

\section{INFLUENCE OF THE CEREBRUM}

I. Effect of removal of the cerebrum.--Removal of the cerebral hemispheres in the guinea-pig leads to somewhat increased gastric tonus and hunger contractions (Dr. King). In the pigeon this 
operation does not change the hunger contractions of the empty crop, except that visual and auditory stimuli do not lead to inhibition of these movements in the decerebrated bird (Rogers). In frogs removal of the cerebrum has no effect on the hunger con. tractions of the stomach (Patterson). Goltz's decerebrated dog showed hunger. We may therefore conclude that in so far as the stomach hunger contractions are dependent upon tonus and motor nervous impulses via the vagi nerves, these impulses do not originate in the cerebral hemispheres.

2. The gastric hunger mechanism during sleep.--In man (infants as well as adults) the gastric hunger contractions are at least as frequent and intense during sleep at night as during the waking state. In our five days' starvation experiment continuous records of the stomach contractions were taken during sleep at night. These records show that the stomach was in strong tonus and hunger contractions practically half of the time of sleeping. The hunger periods were less frequent during the day when the subject was about his work.

Numerous experiments on dogs show that the hunger contractions and the gastric tonus are more vigorous and regular when the animal is sleeping than when he is awake and taking notice of things about him. The only apparent exception to this condition so far observed in any species is the rumen of the goat. A few observations on one goat seemed to show that the hunger contractions of the rumen or first stomach pouch decrease in intensity when the animal is lying down sleeping. We shall not be satisfied that this is so until the same result is obtained on a number of ruminants. Possibly the gastric motor part of the vagi nervous apparatus in the ruminating animals is under a more direct control from the cerebrum than is the case in other species.

During sleep there is decreased activity of the central nervous system in general; decreased tonus of the skeletal muscles; decreased tonus of the musculature of the blood vessels, at least in certain parts of the vascular system; decreased tonus of the urinary bladder, etc.; in short, a lowered activity of all the neuromuscular mechanisms so far investigated. One might have ex- 
pected that so far as the tonus of the empty stomach depends on a central influence by way of the vagi the gastric tonus and hunger contractions would be diminished during sleep. But instead of being depressed in sleep the hunger contractions continue with the same vigor as during the waking state, and in many instances with increased vigor. The increase in the gastric hunger contractions during sleep may be due to the elimination of all inhibitory impulses via the splanchnic nerves. But the absence of depression certainly indicates that the vagogastric tonus mechanism, at least in man and dog, occupies a unique position in the organism-a degree of independence of afferent impulses (exteroceptors) and central processes not known in the case of any other neuromuscular apparatus.

\section{EFFECT OF CEREBRAL STATES (EMOTIONAL STATES, INTELLECTUAL PROCESSES)}

In the dog the cerebral processes of joy, fear, anger, eagerness (for food), attention, etc., cause temporary inhibition of the gastric hunger contractions. This inhibition takes place by way of the splanchnic nerves, not by a depression of the vagus tonus. This, again, points to an unusual independence of the vagogastric tonus apparatus. The sight or smell of food on the part of the starving dog does not initiate or augment the gastric hunger contractions. Dr. Luckhardt has recently shown that when the sleeping dog dreams the gastric hunger contractions are inhibited in the same way that cerebral and emotional processes tend to inhibition of the contraction when the animal is awake.

In man intellectual processes (attention, reading, figuring, arguing) have no distinct influence on the course of the hunger period. Actual anxiety causes temporary inhibition (probably through the splanchnics). We have not been in a position to make observations on the effect of actual anger, fear, and joy, but there is no reason to believe that these processes act differently in man from that in the dog. In man we have paid particular attention to the effects of seeing and smelling palatable food, as it seemed a priori reasonable that the impulses generated by these stimuli 
might make more intimate connection with the vagogastric tonus apparatus. Cannon assumes a "pyschic gastric tonus" analogous to the "psychic secretion" of gastric juice. Glücksmann states that the borborygmi are increased in rate and intensity on seeing and smelling palatable food. He ascribes this to increased gastric contraction. Extensive experiments on Mr. V. and on the author seem to show that this is not the case. These stimuli neither initiate nor augment the gastric tonus and hunger contractions; so far as they influence them at all, it is in the direction of inhibition. One of the tests on the author might be given. Before beginning the five days' starvation period, our colleague, Dr. Luckhardt, was asked to bring in, unknown to the author, a tray of choice food in the midst of a hunger period. The arrangements being made the matter was dismissed from the author's thoughts. At one o'clock on the morning of the fourth starvation day the subject was asleep and the record showed him to be in the midst of a period of vigorous and regular hunger contractions. He was awakened to behold Dr. Luckhardt and the assistant enjoying a feast of porterhouse steak with onions, fried potatoes, and a tomato salad. The tray of edibles was placed not more than 4 inches from the subject's face, and the delicious odor of the food filled his nostrils. He felt the hunger pangs as unusually intense, and there was considerable salivation. However, the gastric hunger contractions were not increased either in rate or intensity. In a few minutes, on the contrary, the hunger contractions became weaker and the intervals between them greater, and the period terminated by this gradual depression much sooner than it probably would have done in the absence of the dinner scene. This was undoubtedly due to local acid inhibition from copious secretion of appetite gastric juice.

When the hungry individual sees or smells good food the gastric hunger pangs are more intense, although there is no change or even when there is some decrease in the strength of the gastric hunger contractions. This is therefore a phenomenon of central reinforcement.

Our data on normal men and dogs seem incapable of any other interpretation than that the vagogastric tonus apparatus, so far 
as it concerns the empty stomach, occupies a unique and physiologically isolated position in the way of nervous control, while the inhibitory apparatus via the splanchnic nerves is readily influenced by central and reflex processes. We feel, however, that these observations must be extended to other groups of vertebrates as well as to such pathological cases in man in which there are indications of abnormalities of the vagogastric tonus before final explanations are attempted or speculation indulged in as to the usefulness of this physiological isolation.

This evidence for the physiological isolation of the hunger mechanism in the way of positive cerebral or central control is of interest in connection with the view that the cravings of hunger and appetite are subjective and largely a matter of habit, and that the periodicity or intensity of these cravings may be altered almost at the will of the individual. Chittenden states this view as follows:

The so-called cravings of appetite are largely artificial and mainly the result of habit. Anyone with a little persistence can change his or her habits of life, change the whole order of cravings, thereby indicating that the latter are essentially artificial and have no necessary connection with the welfare or needs of the body. The man who for some reason deems it advisable to adopt two meals a day in place of three or four at first experiences a certain amount of discomfort, but eventually the new habit becomes a part of the daily routine, and the man's life moves forward as before, with perfect comfort and without a suggestion of craving or a pang of hunger.

Our studies of the hunger mechanism seem to show that the foregoing view is essentially wrong. " In the normal individual the gastric hunger periods begin as soon as the stomach is empty and continue (in the absence of inhibitory processes) as long as the stomach is empty, irrespective of the time of day or night, and without reference to the time the individual is accustomed to eat. In individuals accustomed to the usual three meals in daytime and to sleep during the night the gastric hunger periods are more frequent and usually more vigorous during the night (in sleep) than during the day, provided, of course, the stomach is empty. In the normal individual the empty stomach exhibits periodic hunger activity, and there is no evidence to show that this primary automatism of the empty stomach is in the least influenced by 
eating one or by eating five meals a day. The basis for the view that the time of appearance of the "cravings of hunger" can be changed at will is probably to be sought in the fact that the milder hunger contractions do not enter consciousness as pangs of hunger if the individual's attention is directed into other channels. They are felt as hunger pangs if the individual's attention is directed toward food and eating. The attention is thus directed, consciously or subconsciously, about the time the individual is accustomed to eat. The periodicity of this subjective attention to the milder hunger cravings can probably be altered by training. But this applies only to relatively mild pangs of hunger. The more severe "cravings of hunger" caused by the gastric hunger tetanus rise above the limen of consciousness, except in deep sleep or under conditions of cerebral process involving intense interest. When an individual who is accustomed to eat three times a day turns to a regimen of one meal a day, the quantity of food ingested in that one meal is much greater than that at any one of the three meals a day regimen. The emptying of the stomach and the appearance of the pangs of hunger are correspondingly delayed. The view that prompt appearance and the persistence of the gastric hunger activity in the empty stomach have no relation to the actual need of the individual for food cannot be seriously maintained for the normal animal.

III. INFLUENCE OF THE LOWER BRAIN CENTERS (MID-BRAIN MEDULLA) ON THE GASTRIC HUNGER CONTRACTIONS

The most direct and at the same time the least objectionable method of attack on this problem is the section of the extrinsic nerves to the stomach, although this operation abolishes not only all direct influences from the brain of a motor or inhibitory type, but also the central reflexes (motor or inhibitory) that may be called into action through the sensory nerves in the stomach. The splanchnic nerves were sectioned through a median incision. The vagi nerves were sectioned 2 to $3 \mathrm{~cm}$. above the diaphragm, thus leaving the fibers to the esophagus, the heart, and the lungs intact. 
I. Complete section of the splanchnic nerves.-Observations were made on five dogs with complete sections of the splanchnic nerves on both sides. The longest period of observation after the splanchnic section was two months. Observations were in some cases begun two hours after the operation. When the records of these five dogs are viewed as a whole, it is clear the complete section of the splanchnic nerves in dogs increases the gastric tonus and augments the gastric hunger contractions. The hunger contractions become more rapid and continuous, that is, there is less evidence of the periodic groups with intervening periods of relative quiescence. It is not uncommon to observe contractions at the rate of about two per minute during an entire observation period of 2 to 4 hours. The section of the splanchnic nerves does not abolish the periodicity completely, however. It seems to be a question of relative degree of gastric tonus. If for any reason the tonus of the empty stomach is relatively low on any day, the hunger contractions are less frequent, and there is greater evidence of periods of relative quiescence. We desire to emphasize the fact that this conclusion is based on the observations as a whole. Even the dogs with the splanchnic nerves sectioned showed on some days no greater tonus of the empty stomach and no greater rate and persistence of the gastric hunger contractions than does the dog with these nerves intact. And occasionally a dog with the splanchnic nerves intact exhibits as great a degree of gastric tonus and rate and persistence of the gastric hunger contractions as the maximum observed in dogs with the splanchnic nerves cut. This is to be expected, as by section of these nerves one eliminates only one (and in the normal animal probably one of the least important) of the factors in the motor activity of the empty stomach. The conditions that affect the stomach through the blood and through the vagi are still subject to practically the same variations as in the animal with the splanchnic nerves intact.

After complete section of the splanchnic nerves the psychic or reflex inhibition of the gastric hunger contractions is greatly diminished. The stimuli that cause anger, fear, pain, joy, or pleasure no longer lead to complete cessation of the hunger contractions. 
The maximum effect is a slight and transitory weakening of the contractions. It is therefore evident that the inhibitory fibers in the splanchnic nerves (and possibly also the secretory fibers of the adrenals) constitute the main efferent path in this type of inhibition. The slight degree of inhibition usually in evidence after section of the splanchnic nerves must be due to some central inhibition of the vagus tonus or to action of the few inhibitory fibers in the vagi.

Particular attention was given to the effect of seeing and smelling food on the hunger contractions in these dogs with section of the splanchnic nerves, in order to determine whether these stimuli augment the tonus of the vagi and thus increase the hunger contractions. The results were negative. Even with the greater part of the extrinsic inhibitory fibers to the stomach eliminated, the sight, smell, and taste of food not only fail to inhibit or augment the gastric hunger contractions, but, so far as these stimuli affect the stomach at all, it is in the direction of inhibition of the hunger movements. The apparent increase in the intensity of the hunger pangs in man on seeing or smelling palatable food must therefore be essentially a central phenomenon of "facilitation" or Bahnung.

2. The section of the vagi.-Section of both vagi in the chest was made in three dogs, and after this operation observations on the gastric hunger contractions were continued for from two weeks to three months. Observations were made in some cases two hours after the vagi section.

Section of the vagi leaves the empty stomach on the whole permanently hypotonic; that is, at least for a period of up to three months after the operation. The tonus of the empty stomach in these dogs varies somewhat from day to day, and occasionally the tonus may approach that of a dog with the vagi intact, but on the whole the tonus is permanently much lower than normal. This is evident, not only from the observations by means of the balloon in the gastric cavity, but also on direct inspection and by palpation (introducing the finger through the fistula).

The hunger contractions of the empty stomach are changed mainly in rate and regularity. The duration of each individual contraction is about normal, or on the whole less than normal. 
The long-drawn-out contractions or tetanus are rarely seen. But the intervals between the contractions vary on the whole from 2 to 5 minutes or even up to 8 minutes. The strength, or rather the amplitude, of the individual contractions may appear greater than normal, evidently because the contractions start rather suddenly and without any marked preliminary increase in tonus, and the maximal contractions are evidently so complete that all the air is forced out of the balloon. These contractions may continue of fairly uniform amplitude and rate for 2 to 3 hours, that is, during a whole observation period. The contractions vary in strength and rate from day to day, and on some days they may be completely absent during the entire observation period ( 2 to 4 hours).

The periodicity of the hunger rhythm is, on the whole, obscured, except on the days when the gastric tonus approached that in normal dogs. On such days the contractions appear at shorter intervals, and tend to fall into groups similar to those in normal dogs. Periods of gastric hunger contractions of normal rate and intensity have been observed as early as I 2 hours after complete section of the vagi in the chest. The period of most powerful hunger contractions so far observed in any dog was recorded in one $\operatorname{dog} 24$ hours after the vagi section. The dog had during the four weeks preceding the vagi section showed almost invariably the type II rhythm. It was therefore a dog with unusually intense gastric motor activity. The complete section of the vagi causes on the whole less depression in dogs that exhibit great hunger contractions while the vagi are intact. The variations in the rate and intensity of the gastric hunger contractions in different dogs are therefore primarily due to individual variations in the condition of the stomach rather than to variations in the central innervation or the central inhibition.

In the dogs with the vagi sectioned, but the splanchnic nerves intact, the "psychic" or reflex inhibition of the gastric hunger contractions is still in evidence, but the inhibition appears not to be so marked as when the vagi are intact. Accurate comparisons are, however, difficult to make because of the lowered tonus and the usual long intervals between the hunger contractions after 
section of the vagi. We expected an augmentation of the inhibition through the splanchnics after the vagi section. Instead of finding this to be the case; there actually appeared a gradual diminution in the influence of the splanchnic nerves on the empty stomach in the dog observed for 3 months after section of the vagi. It was not due to the regeneration of the vagi fibers, and consequent restoration of the vagus tonus. If further work should establish this as a fact, we should have a significant instance of physiological readjustment - either an actual diminution in the inhibitory impulses through the splanchnics in consequence of a dynamic readjustment in the central nervous system, or else an increased resistance ("tolerance") to the splanchnic impulses on the part of gastric motor mechanism. It is also evident from this experiment. that if adrenalin is a factor in the inhibition of the gastric contractions via the splanchnic nerves, section of the visceral branches of the vagi leads to a decreased output of this substance from the adrenal gland under the influence of the splanchnic nerves.

3. Section of both splanchnic and both vagi nerves.-Complete sections of the splanchnic and vagi nerves were made on four dogs and observations made on the gastric hunger contractions for 30 to 60 days after the operation. The sections of the splanchnic nerves were made 7 days after the section of the vagi. After this complete isolation of the dog's stomach from the central nervous system, there is practically a permanent hypotonus of the stomach except under conditions of prolonged starvation. The gastric hunger contractions are much the same as when the vagi alone are severed. The contractions are usually of great amplitude, but the intervals between the contractions are frequently longer than in normal dogs. The grouping of the contractions in periods is usually in evidence. These contractions of the isolated and empty stomach were present ro to 20 hours after the vagi section, and there was some improvement in the rhythm or an approach toward the normal tonus and contraction rate during the 30 to 60 days of observation. On the whole, the hunger contractions of the isolated stomach conform to type I. The type II is rare except during prolonged starvation. Short periods ( 2 to 3 minutes) of incomplete tetanus are frequently 
seen, especially during prolonged starvation, and during the first half of the hunger period. It is therefore clear that all the essential characteristics of the hunger contractions of the empty stomach are determined by the local gastric mechanisms rather than by the character of the central innervation or the central inhibition.

Cannon has reported observations on the effects of vagi and splanchnic section on the gastric movements of digestion in cats. Section of the splanchnic nerves did not affect the movements of digestion; section of the vagi caused slowing and weakening of the peristalsis of digestion, but the normal rate of peristalsis was practically restored in a few days. Combined vagi and splanchnic section left the digestive movements of the stomach practically normal, even shortly after the operation. It seems that section of the vagi or complete section of the vagi and the splanchnic nerves in dogs causes on the whole a greater change in the movements of the empty stomach than does the same lesion in cats in case of the movements of the filled stomach. This probably means that the tonus of the vagi plays a greater rôle in the movements of the empty than in the movements of the filled stomach. For it is not likely that there is such marked difference in the relative importance of the vagi nerves in cats and dogs.

The changes in the character of the gastric hunger contractions after isolation of the stomach from the central nervous system seem primarily due to the persistent hypotonus. This is indicated by the fact that on days when the stomach of a normal dog shows relatively slight tonus the hunger contractions approach the type shown by the isolated stomach, and on days when the isolated stomach exhibits tonus approaching that in normal dogs the hunger contractions tend to assume the normal type. Occasionally records are obtained from the empty and isolated stomach that practically demonstrate the above point. During a period of relatively slow hunger rhythm the tonus for some unknown reason may increase markedly for periods of varying length, and during these periods the hunger contractions are identical in rate and character with those of the intact stomach in normal (strong) tonus. In one of the dogs with the vagi and splanchnic nerves sectioned six days 
of fasting led to the appearance of periods of very great gastric tonus, and during these periods (virtually periods of incomplete tetanus) the gastric contractions assumed the form of type III.

However, the details of the changes in the hunger rhythm after isolation of the stomach from the central nervous system seem of minor importance in this connection. The essential point is that since the empty stomach, completely isolated from the central nervous system, does exhibit the typical hunger contractions, the primary rôle of the gastric nerves is that of modifying or regulating essentially automatic mechanisms in the stomach wall. In other words, the extrinsic nerves to the stomach play a rôle similar to that of the nerves to the heart in the regulation of the heart rhythm. Further analysis of the hunger mechanism must be directed primarily to the intrinsic neuromuscular apparatus of the stomach, and secondarily to the factors that control the vagus tonus. 


\section{CHAPTER XI}

\section{THE NERVOUS CONTROL OF THE HUNGER MECHANISM}

(Continued)

\section{INHIBITION FROM THE MOUTH IN MAN AND OTHER ANIMALS}

Our gastric fistula man, Mr. V., offers an exceptional opportunity for studying the relations of certain conscious states, particularly those associated with foods and with eating, on the activities of the empty stomach. The esophagus is completely closed at the level of the upper end of the sternum, so that nothing can enter the stomach from the mouth. The swallowing mechanisms are normal, and the man can swallow and hold in the esophageal pouch about 25 c.c. of material. The gustatory (and olfactory) sense is normal. The senses of thirst and hunger are normal. He masticates his food in the usual way, and the chewing processes are accompanied by the normal conscious states. The masticated food is placed in a syringe and introduced into the stomach through the fistula, which does not involve any pain or discomfort, and the man is adjusted to this condition, as this has been his method of feeding for the last twenty years. Because of the ample size of the gastric fistula the man may sit down at the dinner table, see, smell, taste, and chew his food in the usual manner up to the point of introducing the food through the fistula, while tracings are being taken of a tonus and the movements of the stomach, and records made of the secretion of the gastric juice.

We know, particularly through the researches of Pavlov on dogs, and from many observations on man, that when appetite is present the sight, smell, taste (especially taste) of palatable foods causes a reflex secretion of gastric juice, the so-called "psychic secretion." The efferent nerve-fibers for this reflex reach the stomach through the vagi. The more recent work of Cannon and others has demonstrated that the tonus of the stomach musculature is also primarily dependent on efferent nervous impulses through 
the vagi. A certain degree of tonus is normally a prerequisite for peristalsis or contractions in the empty stomach. The suggestion is therefore obvious that the same stimuli which lead to psychic secretion of gastric juice may at the same time cause an augmentation of the tonus and the contractions of the stomach musculature. Cannon postulated such a "psychic tonus," but no evidence for its existence has been recorded.

It is a universal experience of normal persons that the sight or smell (or even the memory) of palatable foods seems to induce hunger and appetite, or intensify these sensations if they are already present. The simplest explanation of this fact would be that the smell or taste of palatable foods initiates or augments the stomach contractions, thus increasing the hunger sensation by increasing the intensity of the gastric stimulation. The facts, at least in man and dogs, are the very opposite of those demanded by this hypothesis.

There are two sources of error in experiments of this character. In the first place, the periods of contraction of the empty stomach vary in intensity and duration, and the intervening periods of relative quiescence vary in length. The periods of quiescence may be interrupted by occasional contractions. This being the case, the initiation of stomach contractions simultaneously with tasting palatable food during quiescence of the stomach, for example, may be, a mere coincidence. An augmentation of the contractions seemingly due to tasting food during a contraction period may simply be the usual increase in strength of the stomach contraction during such period. In the same way, if tasting food toward the end of a contraction period should be followed by cessation of the stomach contractions, this apparent inhibition may be a coincidence, the cessation of the contractions being "spontaneous" and not casually connected with the tasting of food. These difficulties were realized before the work was undertaken, as it was preceded by an extended survey of the "spontaneous" stomach movements when not interfered with experimentally. Because of the variability of the "spontaneous" stomach activity, the individual test must be repeated a great number of times, and little or no significance can be ascribed to exceptional results. 
A source of error, more serious because not so readily controlled, lies in certain subjective states of an inhibitory character. Pavlov found that while the sight and smell of palatable foods ordinarily caused "psychic" secretion of gastric juice in dogs when hungry, if the dogs knew from past experience that they were not to be permitted to eat the food, the same stimuli caused no secretion. We may have analogous conditions in regard to the stomach tonus and movements. It is possible that, no matter how great the hunger or appetite in man, the knowledge that the seeing, smelling, or tasting food was part of an experiment might initiate cerebral processes of an inhibitory character. This source of error has been controlled in two ways: (I) in Mr. V.'s case the mastication or tasting food was made part of his ordinary routine in preparing the food to be put into the stomach, and the man knew that as soon as the food was prepared it would be introduced into the stomach in the usual way; (2) records were made of the presence or absence of the psychic secretion of gastric juice. If the tasting and chewing of food start a copious flow of gastric juice, we can infer that the tasting and chewing do not give rise to cerebral processes of an inhibitory character.

I. Inhibition of the hunger contractions of the empty stomach by stimulation of the gustatory end organs in the mouth.-The substances used for stimulation were sugar (solid and in solution), quinine in weak solution, sodium chloride (solid and in solution), weak solutions of acetic and hydrochloric acids. Tests were made at all stages of activity of the empty stomach. The results were uniform and practically identical for the four kinds of stimuli employed. If the substances were used in sufficient concentrations to affect the stomach activity, the effects were inhibition of the tonus and contractions. These inhibitory effects follow promptly on placing the substances in the mouth, and disappear, on the whole, very soon after removing the substances from the mouth and rinsing the mouth with warm water. Quinine and the acid produced the longest inhibitory after-effects, probably because of the difficulty in completely removing these substances by rinsing the mouth with water. 
This gustatory inhibition is, on the whole, proportional to the strength of the stimuli (i.e., the concentration of the substance) and varies inversely with the degree of the stomach activity. Thus a weak solution of acetic acid that produced distinct inhibition during the first stage of a period of hunger contraction when the individual contractions are relatively weak may have little or no effect when placed in the mouth during the tetanus stage of the contractions.

If the gustatory stimuli are weak and allowed to act in the mouth for 5 to I 5 minutes, the stomach "escapes" from the inhibition gradually. This is practically true of sweet (sugar). Moderate strength of acids and quinine may hold the stomach in nearly complete inhibition up to $\mathrm{r}_{5}$ minutes. The stimulating substances are, of course, gradually diluted by the secretion of saliva.

Are these gustatory inhibitions primary and relatively simple reflexes independent of the states of consciousness, or are they of the type of conditional reflexes, and therefore due to cerebral states of unpleasant affective tone? This question must be answered by experiments on lower animals with less development of the cerebrum and especially on decerebrated mammals and on so-called "acephalic" infants.

2. Inhibition of the tonus and the contractions of the empty stomach by chewing indifferent substances.-We have been unable to obtain any definite evidence of inhibition of the stomach movements by the movements of mastication when the mouth is empty. But chewing what may be called indifferent substances, such as paraffin, gum, or straw, produces distinct inhibition. Most of the experiments were made by chewing paraffin. Most people can chew paraffin without any sensation of a disagreeable or unpleasant tone, or of a pleasant tone, either, for that matter. Mr. V. said he "did not care for the paraffin," naturally. But he has no dislike for it. The chewing of. indifferent substances produces, on the whole, less inhibition than do gustatory stimuli. The stomach "escapes" from the inhibition in a few minutes, even though the chewing is continued with uniform vigor. The chewing usually fails to produce any effects in the tetanus stage of the stomach activity. 
Inasmuch as the masticatory movements do not cause inhibition if the mouth is empty, we may conclude that inhibition produced by chewing indifferent substances is initiated by mechanical stimulation of afferent nerve-endings in the mouth.

3. Inhibition of the tonus and the hunger contractions of the empty stomach by chewing palatable foods when hunger and appetite are present.-Tests were made with all food substances palatable to Mr. V. and during all stages of gastric tonus and contractions, which imply all degrees of hunger and appetite. But most of the experiments were made with meats in the form of stews, fricassees, or pot roasts, fried eggs, and crackers or bread soaked in milk, soups, or meat gravy. The results are uniform without exception. Chewing or tasting palatable foods inhibits the tonus and the movements of the empty stomach. The inhibition is in evidence within a few seconds after placing the food in the mouth, and may or may not continue for some time after removing the food from the mouth and rinsing the mouth with warm water. The inhibition is least in evidence during the hunger tetanus. In fact, we are uncertain whether the chewing of palatable foods is able materially to affect the stomach in hunger tetanus. It is difficult to determine whether cessation of the hunger tetanus that follows-usually not very promptly - the placing of palatable food in the mouth is a "spontaneous" cessation, or due to inhibition from the mouth. The records show, however, that so far as the stimuli in the mouth affect the processes of the hunger tetanus, the influence is in the direction of inhibition.

The inhibition of the motor activity of the stomach by chewing palatable foods does not appear to have any after-effects in the nature of increased tonus or contractions. Some of the tracings do suggest a motor after-effect, but we are inclined to interpret them in a different way. These effects are obtained only when the tests are made during the relative quiescence of the stomach or at the beginning of a contraction period ("30-seconds rhythm"). Moreover, these results were not always secured even during these periods. It would therefore seem that these apparent augmentary after-effects represent the "spontaneous" initiation of a contraction 
period, or the gradual increase in the magnitude of the contractions characteristic of the periods of the 30-seconds rhythm.

4. Factors involved in the inhibition of the contractions of the empty stomach by palatable foods in the mouth.-Boldyreff has reported that the contractions of the empty stomach in the dog cease during the periods of "spontaneous" secretion of gastric juice. We know that tasting or chewing palatable foods leads to reflex or "psychic" secretion of gastric juice in mammals (including man). May not the inhibition described above be an indirect one, due to the secretion of gastric juice, rather than a reflex inhibition of more direct character? This question has been investigated and settled. A rapid secretion of gastric juice is associated with cessation, partial or complete, of the stomach contractions in $\mathrm{Mr}$. V. We shall show later that this is due, not to the processes of secretion, as such, but to acid stimulation of nerve-endings in the mucosa. When the chewing or tasting of palatable foods leads to copious secretion of gastric juice, this gastric juice is one factor in the accompanying inhibition of the stomach movements.

We know, from Pavlov's work on dogs, that the latent period of the "psychic" secretion is about 5 minutes. The latent period of the "psychic" secretion in man is shorter ( 2 to 3 minutes). The inhibition of the stomach tonus and movements follows within a few seconds after placing the food in the mouth. Hence it is not an acid inhibition from the stomach. The same thing can be shown when the tasting or chewing of the food produces only a scanty secretion of gastric juice. The inhibition appears in the normal way, and the concentrations reappear on removing the food from the mouth despite the slow secretion of gastric juice.

It seems that a certain quantity of gastric juice must accumulate in the stomach or the free hydrochloric acid in the stomach must reach a certain concentration before the acid inhibition takes place. Thus, if the period of chewing or tasting the palatable food is short (4 to 6 minutes), the stomach contractions may reappear at the end of the stimulation in the mouth, and shortly afterward again be inhibited by the acid gastric juice. This inhibition continues during the phase of rapid "psychic" secretion. When the psychic 
secretion is more copious, the reflex inhibition from the mouth merges into the acid inhibition from the stomach.

5. Inhibition of the tonus and the hunger contractions of the empty stomach by swallowing movements. - It has been shown by Cannon and Lieb for the dog that the movements of swallowing lead to a temporary inhibition of the tonus of the stomach. This inhibition is designated the "receptive relaxation" of the stomach. This inhibition is readily demonstrated in man. When Mr. V. makes repeated swallowing movements with only enough saliva in the mouth to initiate the swallowing reflex, a prompt but transitory inhibition of gastric tonus and contractions is produced. The reader will recall that the swallowed saliva does not reach the stomach, but collects in the esophagus pouch. Complete inhibition of the stomach contractions was never secured through the swallowing act, and when the stomach is in the condition of hunger tetanus, or in very strong and rapid contractions bordering on tetanus, the mere swallowing movements seem to have no effect on the stomach. The inhibition of the stomach tonus due to the act of swallowing is most readily demonstrated at the beginning of a period of hunger contractions.

6. Relation of the reflex inhibition of the tonus and the movements of the empty stomach from the mouth to the sensation of hunger.-The stimulation of the gustatory end organs in the mouth, the chewing of indifferent substances, and the tasting and chewing of palatable foods would abolish the sensations of hunger to the same degree that these measures inhibit the stomach contractions. The inhibition of the stomach activity and the cessation of the hunger pains run parallel. This conclusion is based on experiments on a number of men besides Mr. V.

In the dog, food or other substances in the mouth cause inhibition of the hunger contractions of the stomach. But since these manipulations disturb the animal and induce salivation, and in many cases swallowing movements, the precise mechanism of the inhibition must remain in doubt until it can be investigated on dogs from which the cerebrum has been removed, since most of the dog's cerebral processes (pleasant or unpleasant) induce the same inhibition. 
In the rabbit, the sight, smell, or taste of food, or the chewing (without swallowing) of such foods as cherries, carrots, apples, carrot leaves moistened with sugar, acid, or quinine do not inhibit the stomach contractions (Rogers). The same is also true for the guinea-pig (Dr. King). In the case of the single goat so far studied, the chewing of ordinary food (hay, oats, carrots) appeared to increase rather than decrease the hunger contractions of the rumen.

In the pigeons, Rogers encountered the same difficulties that we met in the dogs. Any disturbance of the normal pigeon inhibits the hunger contractions of the empty crop. And since it is not possible to put food or other substances in the mouth of these birds without more or less disturbance by the handling, we cannot be sure that the resulting inhibition proceeds from stimulation of nerves in the mouth. In the decerebrated bird, visual and auditory stimuli do not inhibit the crop, but handling the bird, as in feeding or placing anything in the mouth, causes inhibition. If the disturbing factors other than the mouth stimulation could be eliminated, it is likely that the mouth stimulation alone would cause little or no inhibition unless accompanied by swallowing.

In the frog, stimulation of the nerve-endings in the mouth by food substances, acids, or alkalies cause little or no inhibition of the empty stomach. This is true whether the frog is normal or decerebrated (Patterson).

It is thus evident that the marked reflex inhibition of the gastric hunger contractions from mechanical and chemical stimuli acting in the mouth of man is much less in evidence, although not entirely absent in the lower mammals, birds, and frogs. This leads us to suspect that in man and the higher animals, where this reflex is preponderant, it involves conscious cerebral processes. The question could possibly be settled by experiments on infants̀ (normal and acephalic) and on persons in very deep sleep.

II. THE INFLUENCE OF STIMULATION OF THE GASTRIC MUCOSA ON THE HUNGER CONTRACTIONS OF MAN

The character of the periodic and continuous motor activity of the empty stomach in man and other animals has been described. It has also been shown that the contractions of the empty stomach 
give rise to the sensation of hunger or the "hunger pangs" by stimulation of afferent nerve-endings in the gastric mucosa. We have also seen that in man the hunger contractions of the stomach are inhibited, reflexly, by all stimuli acting on end organs of taste and general sensations in the mouth cavity, so that in case of chewing palatable foods when in hunger we have the so-called psychic secretion of gastric juice preceded and paralleled by a psychic inhibition of gastric motility and tonus. It has also been shown that the hunger contractions persist in their essential character after section of the nerves connecting the stomach with the central nervous system. If we are to attempt to determine more specifically the cause of the hunger contractions our attention must be directed to the stomach itself. The contractions of the empty stomach may be due to any one of these four conditions.

\section{CONDITION OR STIMULATION OF THE GASTRIC MUCOSA}

The absence of food means absence of mechanical stimuli and cessation or diminution of the secretion of gastric juice, and hence a diminished acidity. Carbon dioxide may be secreted into the empty stomach and may act as the primary stimulus. Carbon dioxide and other gases may enter the stomach from the intestines and act as stimuli. Succus entericus, pancreatic juice, and bile may enter the stomach and act as the primary stimulus through alkalinity or by means of specific substances, such as the bile acids. The reader will recall that a number of workers maintain that bile facilitates the intestinal movements.

\section{CONDITION OF THE BLOOD, SUCH AS THE RELATIVE CONCENTRATION} OF NUTRIENT SUBSTANCES, TISSUE METABOLITES, AND HORMONES

It is possible that the neuromuscular apparatus of the stomach is specially sensitized to slight variations in these substances. While we recognize the condition of the blood as a possible factor, it does not seem a probable one; in the first place, because the composition of the blood is on the whole more constant than the composition of the tissues, and because in young and vigorous individuals the hunger contractions of the stomach begin as soon as the stomach is empty and while digestion and absorption are still in progress 
in the intestines, so that there can be no lack of nutrient substances in the blood. In view of the relative constancy of the composition of the blood as shown by all past work on the blood serum and blood plasma, the existence of a periodic fluctuation in the concentration of any one substance in the blood parallel with the periodicity of the hunger contractions seems improbable.

\section{MOTOR NERVOUS IMPULSES THROUGH THE VAGI}

It is well known that the tonus of the stomach depends, in part, on impulses from the vagi, and that the stimulation of the peripheral end of the vagi induces strong contractions in the stomach, whether empty or filled with food. It is also known that the stomach is capable of carrying out the movements of digestion to a fair degree of efficiency after section of both the vagi and the splanchnic nerves. In other words, the neuromuscular apparatus of the stomach seems to be primarily automatic as regards the genesis of the movements of the digestion.

The experiment of sectioning the vagi does not prove this point, however. The experiment does prove the plasticity of the gastric motor mechanism. One would expect that the extrinsic gastric nerves bear the same relation to the movements of the filled and of the empty stomach. This phase of the problem cannot be studied in man. If it should develop that the periodic hunger contractions of the empty stomach are caused by periodic discharges through the vagi, the ultimate question of the cause of hunger would again become a problem of physiology of the central nervous system.

\section{PRIMARY AUTOMATICITY OF THE LOCAL NEUROMUSCULAR MECHANISM OF THE STOMACH}

This can be established only by exclusion of the three other possibilities outlined above. A primarily automatic mechanism might still be influenced by the blood, by the extrinsic nerves, and by local reflexes from the gastric mucosa. The periodicity of the automatic activity might be due, not to a parallel periodicity in any essential stimulus, but to some peculiarity in the metabolism of the stomach developed as a special adaptation, similar to peri- 
odicity in other organs. The absence of the hunger contractions during digestion, or possibly the modification of the hunger contractions into the movements of digestion, must, in this case, be due to specific inhibitory or regulatory impulses from the gastric mucosa.

Mr. V. is admirably adapted for determining the relation of stimulation of the gastric mucosa to the hunger movements, as the fistula is large enough to permit the balloon and connecting tube, and a tube for the introduction of liquids and gases, to be placed in the stomach simultaneously. The liquids and gases can be introduced with or without the man's knowledge. Furthermore, the contents of the stomach (fluid and gas) can be withdrawn for analysis at any stage of the hunger movements and without any material disturbance. But the results first obtained on Mr. V. have been abundantly confirmed on other individuals. This can be done by simply introducing a small tube into the stomach in addition to the balloon with tube connection, so that substances can be put into the stomach without touching the mouth or esophagus.

I. The action of water. -Water, at body temperature, or nearly ice cold, inhibits the tonus and the hunger contractions of the stomach. The inhibition following the introduction of a glass of water (100 to 200 c.c.) directly into the stomach lasts on the whole only 3 to 5 minutes, and is never followed by any augmentation of the tonus or the hunger contractions. The cold water causes greater inhibition than the water at body temperature. If the water is introduced into the stomach during very intense hunger contractions ("hunger tetanus"), there may be no perceptible inhibition. In other words, the degree of inhibition by water in the stomach is inversely proportional to the intensity of the hunger contractions present at the time the water is introduced. Water, warm or cold, introduced directly into the stomach during the period of relative relaxation and quiescence does not increase tonus or initiate a contraction period.

The statement that cold water causes on the whole greater inhibition than water at body temperature requires the following 

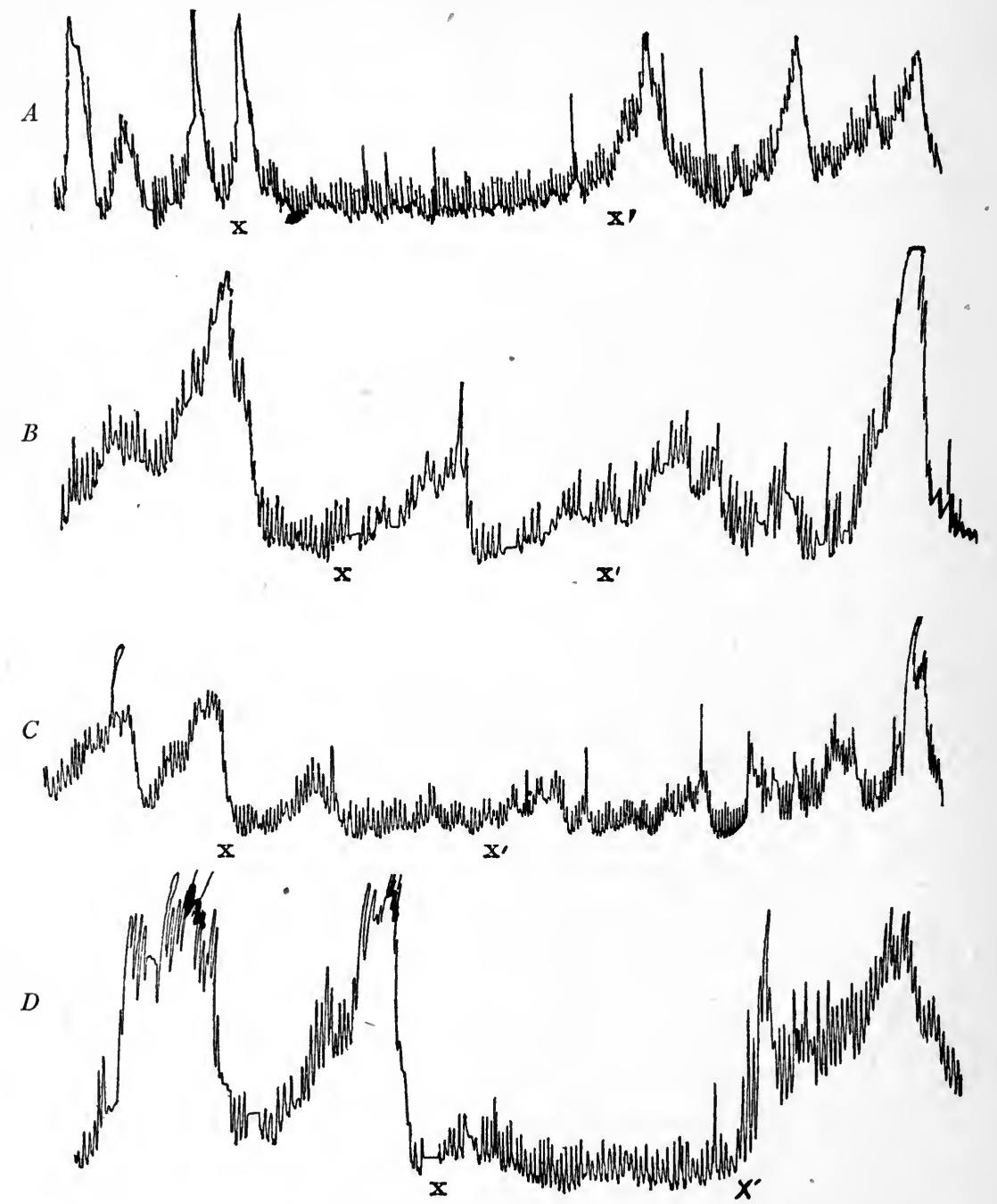

FIG. I8.--Records of gastric hunger contractions of Mr. V. $A$, at $x$ a teaspoonful of sugar was put in the mouth; at $X^{\prime}$ the sugar was rinsed out with warm water; showing inhibition of the stomach contractions by stimulation of end organs for the sense of sweetness. One-half original size. $B$, at $X$ a teaspoonful of table salt was put in the mouth; at $X^{\prime}$ the salt was rinsed out with warm water; showing inhibition of the stomach contractions. One-half original size. $C$, at $X_{15}$ c.c. weak acetic acid was put in the mouth; at $X^{\prime}$ the mouth was rinsed with warm water; showing inhibition of the stomach contractions. One-third original size. $D, X-X^{\prime}$, vigorous chewing of paraffin; showing inhibition of the hunger contractions of the stomach by chewing indifferent substances. 
qualification. The record of the stomach movements was taken by means of an air-inflated balloon in the stomach cavity. Now, when cold water is introduced the water surrounds the balloon, at least partly, and cools the air in the balloon. This itself will lower the tension somewhat, until the temperature is restored to that of the body by the warming of the water or by the passing of the water into the intestine. We do not think that this is a serious
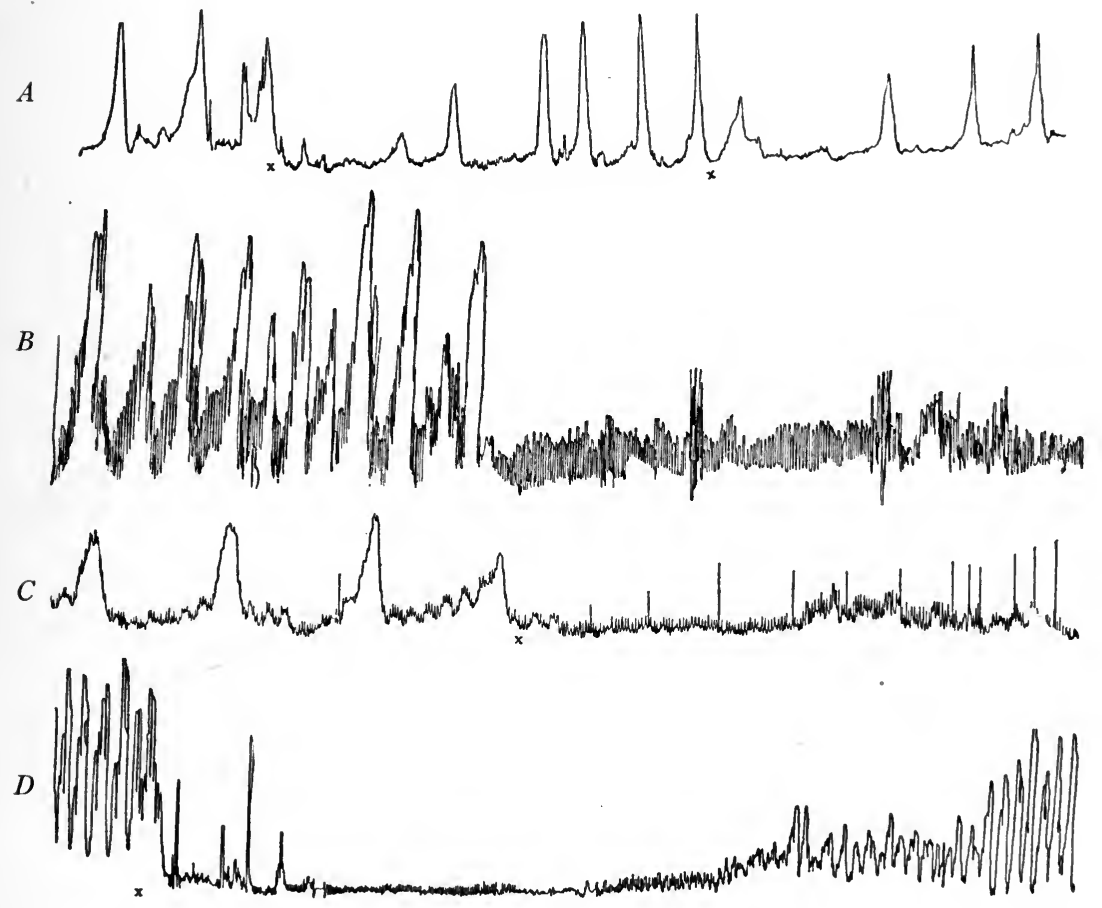

FIG. I9. $-A$, record of the contractions of the empty stomach of Mr. V. At $X$ Ioo c.c. cold water introduced directly into the stomach; showing the temporary inhibition. About two-thirds original size. $B$, records from the empty stomach of A. J. C. At $X$ introduction of 15 c.c. brandy in 25 c.c. warm water directly into the stomach; showing the alcohol inhibition of the hunger contractions. $C$, record of the contractions of the empty stomach of Mr. V. At $X 25$ c.c. of human gastric juice (V.'s own gastric juice, psychic secretion, secured 2 hours previously) introduced into the stomach; showing the acid inhibition. About one-half original size. $D$, record from the empty stomach of dog with gastric fistula. At $X 25$ c.c. of 0.5 per cent $\mathrm{HCl}$ (warm) introduced directly into stomach; showing the prolonged acid inhibition, with gradual recovery. Total time of the part of the tracing reproduced, 30 minutes. About one-half original size. 
source of error for this reason. A few experiments were made with water at $50^{\circ} \mathrm{C}$. This causes greater inhibition than when the water is at $38^{\circ} \mathrm{C}$. Water at $50^{\circ} \mathrm{C}$. will, of course, increase the air tension in the balloon, yet the inhibition of the stomach tonus and movements is sufficiently marked to mask the effect of slight warming of the air.

How does water in the stomach produce this temporary inhibition? It goes without saying that in these experiments the water was not introduced fast enough to cause contractions by distension of the stomach wall, although this occurred unavoidably in a few instances. The only possible ways that water at body temperature can stimulate the nerve-endings in the mucosa seem to be (I) by mechanical pressure, (2) by osmosis, or (3) by changing the chemical equilibrium of the content of the stomach. Cessation of the inhibition probably marks the passing of the water out of the stomach into the intestine or the addition of sufficient salts to prevent stimulation by hypotonicity. The greater inhibitory action by cold water and by water above the body temperature is evidently to stimulation of the protopathic temperature nerve-endings in addition to those acted on by pressure and osmosis.

It is clear that the action of water on the stomach mucosa is in the direction of inhibition of the hunger contraction. How can this be reconciled with the view that a glass of cold water induces or augments hunger? It is to be remembered that in these experiments the water had no chance to act on the nerve-endings in the mouth and the esophagus. The alleged action of cold drinks on hunger and appetite is probably the reflex effects (cold) from the mouth and esophagus. In the writer's own case a glass of ice water causes increased muscular tonus, sometimes even to the point of shivering and formication. This increased kinesthetic sense probably acts in the way of Bahnung for the hunger sensation, if it is not actually a part of the hunger complex. Cannon and Washburne suggest that the effect of a cold drink on the hunger sensation is due to "the power of cold to induce contraction in smooth muscle." Although their meaning is not clear to us, they probably have in mind the contraction of the stomach musculature. This 
could not come about by the cold acting on the stomach musculature directly. The reflex effects of cold water from the mouth and esophagus are very complicated as regards the stomach, while cold water acting on the gastric mucosa directly causes inhibition, and cooling the frog's stomach causes depression and atony in proportion to the degree of cooling.

2. The action of acids.-All acids, or liquids containing acids, including normal human gastric juice, cause inhibition of the movements and the tonus of the empty stomach when introduced directly into the stomach cavity. No acid has been tested in stronger concentration than 0.5 per cent. The duration of the inhibition is on the whole directly proportional to the concentration and the total quantity of acid introduced; 200 c.c. of 0.5 per cent of $\mathrm{HCl}$ will usually inhibit for a period of 25 to 30 minutes only.

This inhibition by acids can be made evident during all stages of activity of the empty stomach. If the acid is introduced during relative quiescence of the stomach, the appearance of the next period of hunger contractions is delayed; if introduced during the active contractions, these are abolished or depressed.

The duration of the acid inhibition is probably determined by three factors, namely, (I) passing of the acid into the duodenum, (2) fixation and neutralization of the acid of the mucous gastric secretion, (3) neutralization by bile and intestinal juice which at times pass into the stomach through the dilated pylorus.

While it is a striking fact that gastric juice of full normal acidity ( 0.48 to 0.53 per cent) and other acid solutions inhibit the hunger contractions, it does not follow that a neutral or alkaline reaction in the gastric cavity is a prerequisite for these contractions. During the strong contractions the stomach secretes a juice rich in mucin and combined $\mathrm{HCl}$, but usually containing some free $\mathrm{HCl}$. After the introduction of acids the contractions reappear before all the acid has passed out of the stomach or has been completely neutralized. And in case Mr. V. chews palatable food during a strong hunger period, the hunger contractions reappear before there is complete cessation of the psychic secretion of gastric juice. In other words, the hunger contractions are not inhibited by weak 
concentrations of acids in the stomach. A neutral or alkaline reaction of the mucosa is not necessary for these contractions. If the food is sufficiently palatable and the mastication is continued long enough, the inhibition produced reflexly from the mouth fuses with the acid inhibition from the stomach. If the food is not especially palatable or the mastication period brief, the contractions may resume on cessation of the chewing and then again be inhibited for a time during the period of most rapid secretion of the appetite gastric juice.

The degree of inhibition produced by normal gastric juice is the same as that caused by an equal quantity of hydrochloric acid of a concentration equal to the free acidity of the gastric juice. "It would thus seem that the hydrochloric acid in the gastric juice constitutes the stimulus that leads to the inhibition.

This acid inhibition of the hunger contractions is of peculiar interest in connection with the neuromuscular mechanisms of these hunger movements and the gastric movements in normal digestion. In man the movements of the stomach in digestion are not inhibited by acids in the stomach, that is, at least not by acids in concentrations equal to that of the gastric juice. The fact that the intensity of movements of the antrum increases as the gastric digestion advances may even indicate that a certain degree of free acidity facilitates the movements of digestion. At first it occurred to us that since acid in the stomach inhibits the hunger contractions, but not the digestion contractions, the mechanisms involved in these two types of gastric activity are different, at least as regards the character of the afferent impulses from the gastric mucosa. But on further reflection it became apparent that this is not necessarily the case, for the digestive movements involve primarily the pyloric end, while the hunger movements (as studied by our method) involve the fundus of the stomach. It is possible that acid stimulation of the nerve-endings in the gastric mucosa leads, reflexly, to a temporary inhibition of the fundus and to peristalsis of the pyloric region of the stomach.

3. The action of alkalies.-The tests were made with sodium carbonate in concentrations varying from 0.2 to $\mathrm{I} . \mathrm{O}$ per cent, and 
in varying quantities. In concentrations of 0.2 per cent or less the sodium carbonate solution appears to have the same influence on the hunger contractions as equal quantities of water, that is, a slight temporary inhibition. This inhibition is evidently due, not to the alkalinity, but to the bulk of the solution. In concentrations of 0.2 per cent to r.o per cent the degree of inhibition produced is on the whole directly proportional to the concentration and the quantity of the solution put into the stomach; 200 c.c. of I per cent sodium carbonate causes about the same degree of inhibition as 200 c.c. $\frac{1}{2}$ per cent hydrochloric acid. It is thus clear that alkalinity has the same effect as acidity, only to a less degree, both acids and alkalies causing inhibition without any after-effect of the nature of augmentation.

The fact that 0.2 per cent sodium carbonate has no more effect on the hunger movements than equal quantities of water seems to show that a slight alkalinity of the gastric mucosa is compatible with the hunger contractions of the empty stomach. It makes it also evident that the entrance of bile or intestinal juice into the stomach will have little or no effect on these movements, while any concentration that influences these movements produces inhibition.

4. The action of local anesthetics.-Solutions of some local anesthetics were tested with the view of determining whether the sensory nerves in the gastric mucosa play only an inhibitory rôle in the processes of gastric hunger contractions. Phenol, chloreton, orthoform, quinine-urea-hydrochloride, and adrenalin chloride were used in quantities and concentrations compatible with absolute safety to Mr. V. It was not considered advisable to use cocaine. The solutions of the drugs were introduced in quantities of roo to 200 c.c.

In the concentrations employed no specific action of any of the above substances could be determined. For example, roo c.c. of phenol (dilution I to I0,000) has the same effect as Ioo c.c. of water, that is, a slight temporary inhibition. The same applies to the other drugs. No appreciable anesthesia of the gastric mucosa was produced' by any of the drugs. It seems probable that the solutions of these drugs pass out of the stomach just as rapidly as equal 
quantities of water, and hence do not remain long enough in the stomach to produce local anesthesia. Because of the danger attending the use of local anesthetics in strong concentrations in the stomach, this work was not carried further on man. It seemed, however, that adrenalin chloride introduced into the stomach in considerable quantities could not be particularly injurious. But even in large quantities (roo c.c. of a dilution of $\mathrm{I}-\mathrm{I0}, \infty 00$ ) the adrenalin acting in the gastric cavity has no other effect on the hunger movements than equal quantities of water.

5. The action of alcoholic beverages. - Tests were made with sour and sweet wines, beer, brandy, and pure alcohol (diluted). The taking of alcoholic beverages with meals is a habit with many people. It is claimed by many people that a glass of wine, beer, or some mixture of alcohol taken before meals increases the appetite (and possibly the hunger). The writer is neither a total abstainer nor a habitual user of alcoholic beverages. But it is his experience that a glass of beer taken at mealtime seems to awaken or increase appetite. This effect is rather immediate and therefore not due solely to the absorption of the alcohol. Pavlov has recorded an instance from his own experience where a drink of wine seemed to initiate the sensation of hunger the very minute the wine reached the stomach. From inquiries as extensive as opportunities have permitted we are inclined to believe that this"apparent augmentation of hunger or appetite by alcoholic beverages is rather a common experience.' In view of this fact, we expected to find that these alcoholic beverages increased the tonus and the contractions of the empty stomach, since it is the tonus and the contractions of the empty stomach that give, rise to the hunger sensation. To our surprise the results proved to be the very opposite. Wine, beer, brandy, and pure alcohol (diluted) introduced directly into the stomach inhibit the hunger contractions and the tonus of the empty stomach instead of increasing them. This is true, whether these fluids are cold or at body temperature. If these alcoholic beverages are greatly diluted with water, a degree of ,dilution can be reached which has the same action on the empty stomach as equal quantities of water, although the specific beverage is readily 
detected when the mixture is placed in the mouth. In no instance have we been able to make out any undoubted augmentation of the stomach tonus and hunger contractions after the inhibition period. In other words, alcoholic beverages when introduced directly into the empty stomach in quantities and concentrations that directly affect the tonus and the contractions of the stomach cause inhibition, and inhibition only.

The pure alcohol was never used in stronger concentrations than Io per cent. The brandy was usually diluted one-half with water, while the beer and wines were put in the stomach undiluted.

We have seen that acids in the stomach cause inhibition of the hunger contractions. Pure alcohol also causes inhibition. It is therefore evident that the alcohol and acids are primarily responsible for the inhibition following the introduction of alcoholic beverages into the empty stomach. For the sake of brevity we may designate it as "the alcohol inhibition."

The duration of the alcohol inhibition varies directly with the quantity and concentration of the beverage introduced into the stomach. Thus 50 to 100 c.c of ro per cent alcohol may inhibit the hunger contractions for $\mathrm{r}$ to 2 hours; or if introduced during a period of relative quiescence it delays correspondingly the onset of the next hunger period. Inhibition for 30 to 60 minutes is caused by 200 c.c. of beer. The sour wines on the whole cause greater inhibition than the sweet wines, probably through their acids.

It must be stated that these alcoholic beverages were put into the stomach of Mr. V. and the other subjects, including the author, with their consent and without any protest, resentment, fear, or disgust on their part, which might account for the stomach inhibition. Mr. V. takes wine and beer occasionally. At times he bought his own choice of wine and beer and introduced into the stomach the desired quantities. The effect on the hunger contractions was always the same. We are therefore dealing with a characteristic alcohol and acid inhibition, and not with a masked "psychic" inhibition.

How are these results to be harmonized with the seeming stimulation of the appetite by alcoholic beverages taken by the mouth? 
In the first place, the local inhibitory. action of alcoholic beverages in the gastric cavity is so marked and so invariable that we feel confident that this is always the gastric effect of these beverages in man, whether taken normally by the mouth or introduced into the empty stomach without coming in contact with the mouth or esophagus. Alcoholic beverages can therefore not initiate or increase hunger, since hunger is caused by the stomach contractions, and these are inhibited by the alcohol. Since most of the alcoholic beverages stimulate the end organs of taste and smell as well as those of general sensibility in the mouth cavity and in the esophagus, it is possible that this stimulation in some way augments or initiates appetite for food. If this is the case, we have the singular condition of alcoholic beverages augmenting appetite and inhibiting hunger at the same time. There can be little doubt that cerebral states, as modified by training and habit, are also factors in this apparent action of alcoholic beverages on appetite. It is certain that the individual's first taste of alcohol, beer, or sour wines does not focus his attention on food and eating.

If alcoholic beverages in the stomach caused as marked inhibition of the stomach movements in digestion as they do in the stomach movements in hunger, even moderate drinking with meals would lead to acute indigestion. As this is not the case, it is evident that alcoholic beverages affect the mechanism of these two types of movements differently.

6. The action of carbon dioxide and air.-The action of carbon dioxide in the cavity of the empty stomach was studied in two ways: (I) by introduction of water charged with $\mathrm{CO}_{2},(2)$ by introduction of $\mathrm{CO}_{2}$ gas. An excess of carbon dioxide in the blood of the abdominal vessels is supposed to augment the tonus of the digestive tract, although some recent work of Hooker throws doubt on this interpretation of the stimulating action on intestinal peristalsis by partial asphyxia. An excess of $\mathrm{CO}_{2}$ is sometimes found in the gaseous contents of the empty or partly filled stomach. It is known, furthermore, that carbon dioxide in sufficient concentration acts as a powerful stimulus to the nerve-endings in such membranes as those of the mouth and nose and of the cornea and conjunctiva. 
Carbon dioxide in the cavity of the empty stomach was at first considered a possible stimulus to the gastric hunger contractions, but this hypothesis proved entirely erroneous. So far as the carbon dioxide in the cavity of the stomach affects the hunger movements, the influence is in the direction of inhibition.

Water saturated with $\mathrm{CO}_{2}$ under pressure has practically no more effect than similar quantities of pure water. It produces the same degree of temporary inhibition without any after-effect of augmentation. As such carbonated water stimulates the nerveendings in the mouth in the characteristic way, it follows that the nerve-endings in the stomach are less affected by $\mathrm{CO}_{2}$ than are the nerve-endings in the mouth.

When the $\mathrm{CO}_{2}$ is forced into the stomach in the form of gas and under pressure, the results are complicated by the mechanical action of the gas in forcibly distending the walls of the stomach and raising the intragastric pressure, and hence increasing the pressure on the balloon in the fundus. A sudden and forcible distension of the empty stomach, no matter how produced, leads to a few strong contractions. This factor can be fairly well controlled by introducing the gas slowly. When this precaution is taken, the empty stomach can be considerably distended with $\mathrm{CO}_{2}$ gas, without any marked effect, either on the tonus or on the hunger contractions. But the chemical effect of $\mathrm{CO}_{2}$, so far as it is demonstrable at all, is in the direction of inhibition.

It will undoubtedly occur to the reader that this slight inhibition by the $\mathrm{CO}_{2}$ may be an instance of "psychic" inhibition from the distress of an overdistended stomach. This possibility has been guarded against. In the first place, the stomach was not distended to the point of painfulness by the carbon dioxide. Furthermore, the stomach cavity was irrigated, so to speak, with the gas without raising the intragastric pressure perceptibly, by introducing the inlet tube to the cardiac end and allowing the gas to escape by way of the mouth, or, in the case of Mr. V., by the open fistula. Under these conditions the same slight inhibitory effects were recorded without signs of primary or secondary augmentation. It is thus clear that so far as carbon dioxide in the gastric cavity 
affects the gastric tonus and hunger contractions at all the action is in the direction of inhibition. This is probably due to the acid stimulation of the nerve-endings in the mucosa.

The introduction of air into the empty stomach has no effect whatever on the tonus and the hunger contractions, provided the stomach is not overdistended by the air, or the air introduced rapidly and under such pressure as to cause sudden and forcible distension of the stomach walls. This leads to a few contractions. But the same thing is produced by sudden inflation of the balloon in the fundus. It is therefore purely mechanical. Oxygen in greater concentrations than that of the air has not been tried. But it is evident that the 20 per cent oxygen of the air acts neither favorably nor unfavorably on the hunger movements.

The fact that nothing but inhibition is produced by substance acting on the gastric mucosa suggests that this may be in every case a "psychic" inhibition masking any weak action that may be of a positive or augmentation type. The very consciousness that these substances were introduced into the stomach for experimental purposes might be the primary element in this possible psychic inhibition. That cerebral states may inhibit the gastric hunger movements is certain from results both on man and on dogs. In one instance, when preparing to introduce 200 c.c. of 0.5 per cent acidic acid into the stomach in the midst of the period of powerful hunger contractions, Mr. V. somehow thought that we intended to introduce that much concentrated acid (or vinegar). As we were going about with the preparations it was noticed that the stomach contractions suddenly became very feeble. Mr. V. looked worried. We inquired if he did not feel right, and he asked if we intended to put all that vinegar into the stomach. "It will surely hurt me," he said. To assure him, the author drank half of the acid himself, and then asked him to take a mouthful of it. Then he laughed and said, "Oh, I thought it was pure vinegar." In two minutes after the mental stress and anxiety was over the hunger contractions returned to their normal rate and amplitude.

The following facts speak against the possibility of the results being due to psychic inhibition: (I) There was no evidence that 
Mr. V. or any of the subjects were in any way afraid, displeased, disgusted, or impatient with the experiments. (2) The direct proportion between the quantity and the concentration of the substance introduced into the stomach and the degree of inhibition produced is contrary to the hypothesis of a psychic inhibition. The displeasure or disgust ought to have been practically the same on introduction of $0 . \mathrm{I}$ per cent and of 0.5 per cent $\mathrm{HCl}$, of I per cent and ro per cent alcohol, as in most cases the subjects did not know the strength of the material used. (3) In many cases the subject was purposely deceived as to the nature of the material, using water for acids and vice versa. The stomach reaction was invariably in accordance with the substance actually introduced.

We feel satisfied, even on the basis of the tests on man, that psychic inhibition plays no rôle in these results. But to meet the possibility once and for all, we have repeated and confirmed all of the above tests on dogs. The parallel on the two series on man and dog is complete. May not psychic inhibition play a rôle in the tests on dogs? It does not, and for the following reasons: (I) The dogs could not have known either the difference between the substances introduced into the stomach or the different concentrations of the same substance. (2). Tests were made during sleep and without the animal waking up. The results were the same. (3) Psychic inhibition of the gastric hunger movements in dogs is invariably of much shorter duration than the inhibition caused by acids, alkalies, and alcoholic beverages.

It is therefore clear that these results on man are fundamental facts in the physiology of the stomach and not primarily dependent on afferent impulses that enter consciousness.

\section{INFLUENCE OF THE INHIBITIONS FROM THE GASTRIC MUCOSA ON THE} FUNDAMENTAL RHYTHM OF THE GASTRIC HUNGER CONTRACTIONS

During the progress of this work it soon became apparent that these temporary inhibitions described above do not cut short a hunger period, but simply delay its culmination. The contractions that appear as the inhibition ceases are the continuation of the period temporarily checked by the inhibition. They are not the 
beginning of a new period. When the tetanus stage of the hunger period is reached a stimulation of the gastric mucosa sufficiently strong to cause prompt cessation of the contractions seems actually to terminate the period, for when the contractions reappear they are not the incomplete tetanus or strong and rapid contractions of the culmination of the period, but the feeble and slow movements characteristic of the beginning of a period. By careful adjustment of the quantity and strength of the material introduced into the stomach during the first part of the hunger period and by renewing the inhibition on reappearance of the rhythm, it is possible to lengthen a 30- to 40-minute period into a 90- to I 20 -minute period. In other words, the motor mechanisms of the hunger contractions may be compared to the spring of a watch. When the spring is wound up it will run the watch for a certain number of hours, and it makes no difference whether or not these hours are consecutive.

It seems to us that this fact has an important bearing on the question of the primary stimulus to the hunger movements. It seems to point to a primary automatism, peripheral or central, or both, relatively independent of the condition of the blood as well as of the afferent nervous impulses. The fact speaks particularly strongly against the hypothesis that the primary stimulus is to be sought in the condition of the blood. For example, if the primary stimulus is in some condition of the blood, this condition must be present and to a gradually increasing degree from $\mathrm{I} 2: 30$ to $\mathrm{I}: 00$ P.M. to parallel a hunger period beginning at $\mathrm{I} 2: 30$ P.M. and ending at I :०० P.M. And this condition of the blood must be absent from I:०० P.M. to I: 45 P.M., as the stomach is relatively quiescent during that time. The hypothesis seems to be rendered untenable by the manipulations which do not, at least in some cases, involve any change in this hypothetical condition of the blood. The culmination of the hunger period may be delayed till $\mathrm{I}: 30$ or $\mathrm{I}: 45$ P.M., so that the strongest hunger contractions fall in the time when the blood does not stimulate the gastric mechanism in a way to cause hunger movements.

But what is the significance of this inhibition in the normal work of the stomach? The inhibition of the hunger contractions 
by mechanical and chemical stimulation of the gastric mucosa in the normal person prevents the appearance of these contractions during the period of gastric digestion. This negative control of the hunger movements from the stomach cavity is obviously a useful co-ordination. The primary or actual stimulus to the hunger contractions is therefore to be sought in the vagus tonus, in some condition of the blood, or in a primary automatism of the gastric neuromuscular mechanism. We have some evidence that the latter is the essential factor and that extrinsic nerves and the condition of the blood only modify the primary automatism. If this is the case the hunger contractions ought to appear as soon as the stomach is empty of food or other substances capable of stimulating the nerve-endings in the mucosa. We should also expect these contractions to be more or less continuous as long as the stomach is empty, at least in young and vigorous individuals, and when the condition of the individual as a whole does not lead to increased activity of the extrinsic inhibitory nerves (splanchnics). On this hypothesis the gradual tonus contraction of the gastric fundus pari passu with the progress of the gastric digestion represents the algebraic sum of the inherent automatism and the inhibitory effects from the gastric cavity. A gradual fatigue of the inhibitory mechanisms is probably also a factor, as we have abundant evidence (in man and dog) of such "escape" of the stomach from inhibitory nervous processes.

We should probably look for the closest parallelism between the gastric hunger contractions and the absence of stimulation of the gastric mucosa in infants and young children, that is, before cerebral (and possibly gastric) habits relative to feeding have been established. We have made a close study of a healthy (bottle-fed) infant touching this point. It is well known that, other things being equal, the more food put into the stomach the longer is the time required for the completion of gastric digestion. If this infant (five months old) is given only 4 ounces of food he calls for more after about 2 hours. If he is given 7 to 8 ounces of the same food the call for more food is delayed for 3 to 4 hours. If he is given 5 ounces of the food at 6:00 P.M. he nearly always wakes up 
and calls for more at $\mathrm{I} 2: 00$ or $\mathrm{x}: 00$ o'clock, while if he is given as much food as he will take ( $7 \frac{1}{2}$ to $8 \frac{1}{2}$ ounces) at 6:00 P.M. he rarely wakes up and calls for food until 3:00 or 5:00 o'clock the following morning. There is evidently a close parallel between the time of the emptying of the stomach and the appearance of the hunger contractions. The more frequent calls for food during the day are obviously due to the fact that the gastric hunger contractions must reach a certain degree of intensity before they cause the soundly sleeping infant to wake up. This is certainly true in the case of dogs. A dog may sleep on peacefully and quietly during gastric hunger contractions of moderate intensity. When these contractions become very intense the dog moves or moans in his sleep and sometimes wakes up.

While we have made no observations on the action of acids, alkalies, and alcoholic beverages on the gastric movements of digestion in man, it is well known that these substances do not inhibit these movements to the extent that they inhibit the hunger contractions. The movements of digestion are primarily concerned with the pyloric region, while the hunger contractions involve the cardiac and fundus region. Evidently these two regions of the stomach react differently to local chemical stimulation of the gastric mucosa.

In view of the fact that acids as well as normal gastric juice inhibit the gastric hunger contractions, one might expect that persons having gastric hypersecretion should experience little or no true hunger sensations or pangs of gastric origin. At the same time we must consider, in cases of prolonged hypersecretion, the possibility of a readjustment of such a character that the acid stimulation of the mucosa causes less inhibition than is the case in the normal stomach.

III. INHIBITORY REFLEXES FROM THE GASTRIC MUCOSA IN DOGS AND OTHER ANIMALS

The work on man led to the conclusion that any substance capable of stimulating the nerve-endings in the gastric mucosa causes inhibition of the tonus and hunger contractions, and inhibi- 
tion only, as there is no evidence of any increase in the gastric tonus or hunger contractions following the primary inhibition. The experiments on dogs were undertaken primarily to determine the character of this reflex, that is, whether central, or local, or both. The liquids were introduced into the stomach through the fistula by means of a soft rubber tube, so that swallowing acts and the stimulation of nerve-endings in the mouth, the pharynx, and the esophagus were completely eliminated.

I. Action of water, acids, alkalies, and alcoholic beverages.-The observations were made on six dogs with all the extrinsic gastric nerves intact; on six dogs with the splanchnic nerves cut; and on four dogs with complete section of both of the vagi and the splanchnic nerves. The results on the normal dogs are practically identical with those on man. Gastric juice (human, canine), weak acids and alkalies, brandy, wines, and beer introduced directly into the empty stomach during hunger contractions produce immediate inhibition of the gastric tonus and contractions. Thus the same quantity of gastric juice or wine seems to cause more prolonged inhibition in dogs showing the type I than in the dogs showing type III hunger rhythms. The duration of these inhibitions can best be studied in the dogs showing types II and III of hunger contractions, as these two forms are practically.continuous, so that the errors from spontaneous periods of relative quiescence are eliminated. In normal dogs showing contractions of types II and III, 25 c.c. gastric juice or 0.5 per cent $\mathrm{HCl}$ usually causes complete inhibition for 20 to 30 minutes. The return of the hunger contractions is always gradual. In like manner, 25 c.c. of beer will inhibit for $\mathrm{I}_{5}$ to 25 minutes. In one case 50 c.c. of beer caused complete inhibition for one hour.

If these substances are introduced into the stomach of dogs during a period of relative quiescence and tonus relaxation the only effect appears to be a still greater tonus relaxation and prolongation of the quiescent period. In some cases one or two hunger contractions follow immediately on introducing the material into the stomach. We are inclined to attribute these contractions to the mechanical distension of the stomach wall rather than to 
stimulation of nerve-endings in the mucosa. This phenomenon was never observed when the stomach was in strong tonus and hunger contractions.

2. Action of carbon dioxide.-The influence on the hunger contractions of $\mathrm{CO}_{2}$ in the stomach cavity is the same in dog and man. The experiments on dogs were made with water saturated with $\mathrm{CO}_{2}$ and with $\mathrm{CO}_{2}$ gas. When the gas was employed, at times enough of it was passed into the stomach via the fistula to cause escape of the gas through the esophagus. The water saturated with $\mathrm{CO}_{2}$ has practically the same action as ordinary water, that is, a slight temporary inhibition without any after-effect of the nature of increased tonus or contractions. This is true whether the carbonated water is introduced during active hunger contractions or during relative quiescence. The $\mathrm{CO}_{2}$ gas usually initiates some contractions if introduced into the stomach during a period of quiescence. This is evidently due to mechanical distension of the stomach walls and not to chemical stimulation of nerve-endings in the mucosa. If the empty stomach is in vigorous tonus and hunger contractions the $\mathrm{CO}_{2}$ gas causes a slight temporary inhibition without any stimulating after-effect. This temporary inhibition is in all probability due to a weak acid stimulation in the nerve-endings in the mucosa.

3. Effects of complete section of the splanchnic nerves.-The inhibition of the gastric tonus and hunger contractions by acids, alkalies, alcohol, etc., in the stomach cavity persists after section of the splanchnic nerves, but it is on the whole less complete and of shorter duration than in dogs with all the extrinsic gastric nerves intact. This applies to all substances used in this series of experiments. When, as in the present series, the test with each substance is repeated at least ten times on each animal, some variation in the intensity and duration of the inhibition appears. That is to be expected, because the degree of inhibition depends on several variable factors, such as the excitability of the nerve-endings in the mucosa, the excitability of the Auerbach plexus and of the central nervous system, the tonus of the stomach, etc. It is therefore true that the most pronounced inhibition observed after section 
of the splanchnic nerves may be as marked as the feeblest inhibition obtained in the normal dogs. But when all the results in the two series of dogs are compared there is no question but that section of both splanchnic nerves diminishes the inhibition following chemical stimulation of the gastric mucosa by acids, alkalies, alcohol, etc.

Several explanations of this fact suggest themselves. (I) Since section of the splanchnic nerves in dogs increases on the whole the tonus and the hunger contractions of the empty stomach, the diminished inhibition may be due to this greater vigor of the stomach rather than cutting the efferent path of a long reflex. We do not think that this is the main or important factor, because the typical marked inhibition is obtained in normal dogs, even when the stomach shows as vigorous tonus and hunger contractions as the maximum shown by dogs with the splanchnic nerves severed. Moreover, the inhibition is still incomplete in splanchnetomized dogs that show relatively feeble hunger contractions. (2) The substances stimulate afferent vagi nerve-endings in the mucosa, and the afferent vagi impulses via conscious or subconscious centers finally stimulate the efferent inhibitory neurones in the splanchnic system. It is well known that the vagi carry afferent fibers from the stomach mucosa and that the splanchnic nerves carry inhibitory fibers to the stomach. The present experiments give the first intimation that the afferent vagus and the efferent splanchnic systems are so intimately associated in gastric motor reflexes. It is possible that the reflex also involves the adrenal glands, so that the inhibition mentioned above is to be accounted for, in part, by the depressor action of an increased output of epinephrin.

4. Effect of section of the vagi nerves and of the vagi and the splanchnic nerves.-When all the records are compared it appears that section of the vagi nerves alone or section of both the splanchnic and the vagi nerves diminishes the inhibitory reflex from the gastric cavity on the whole more than does the section of the splanchnic nerves alone. A fact of greater importance, however, is the persistence of the reflex after complete isolation of the stomach from the central nervous system. The inhibition is therefore a primary local reflex. The decrease of the inhibition after the vagi section 
may involve two mechanisms. It is well known that the vagi contain some efferent inhibitory fibers to the stomach motor mechanism, and these may be, together with the splanchnic inhibitory fibers, involved in the long inhibitory reflex. But since the gastric

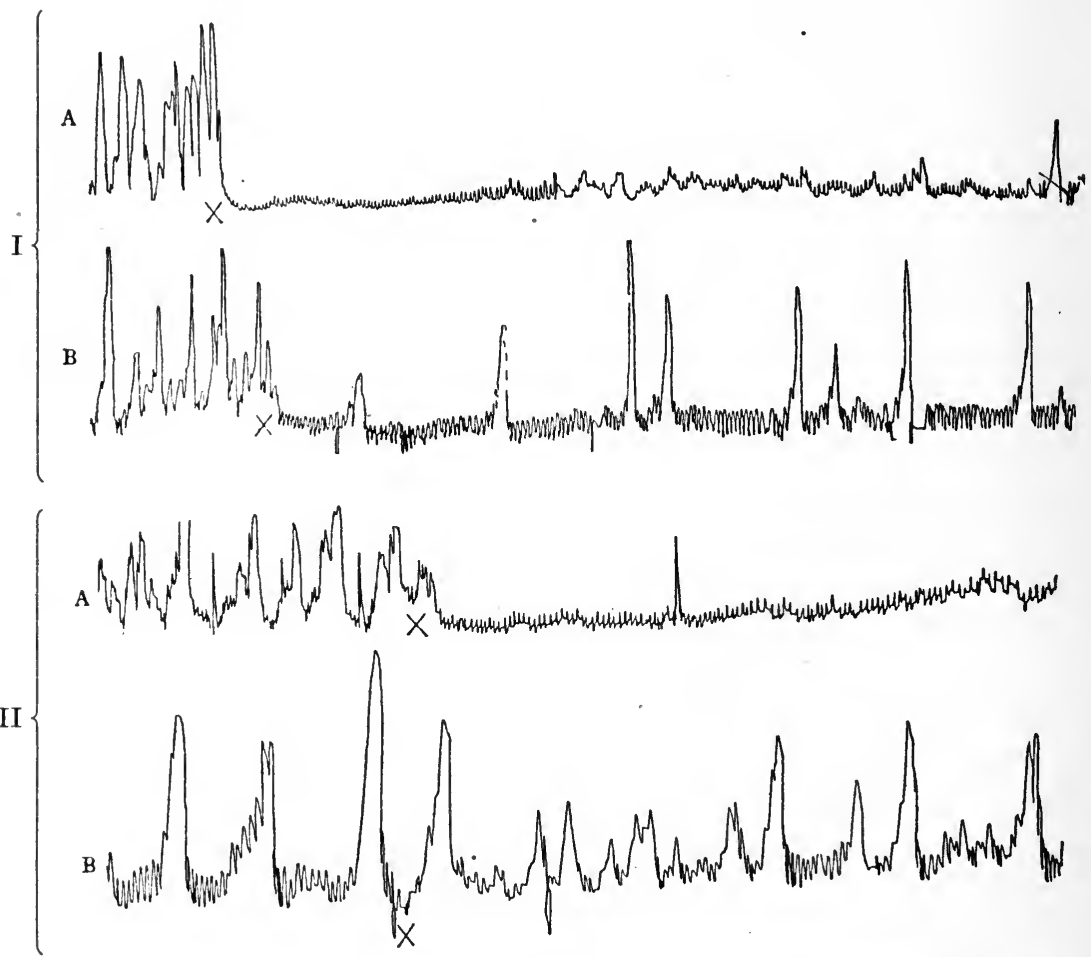

FIG. 20.-I. Tracings from empty stomachs of dogs. $A$, normal dog; $B$, dog with both splanchnic nerves cut; $X$, introduction of 25 c.c. 0.5 per cent $\mathrm{HCl}$ into the stomach; showing less complete inhibition of the hunger contractions by acid in the stomach after section of the splanchnic nerves. One-third original size.

II. Tracings from the empty stomach of dogs. $A$, normal $\operatorname{dog} ; B$, dog with section of the vagi and splanchnic nerves; $X$, introduction of $\mathrm{I} 2$ c.c. of brandy $+\mathrm{I} 2$ c.c. of water into the stomach; showing less complete inhibition by alcohol in the case of the stomach isolated from the central nervous system. One-half original size.

tonus fibers in the vagi and the gastric inhibitory fibers in the splanchnic nerves are practically antagonistic, it is highly probable that afferent influences leading reflexly to the stimulation of the 
inhibitory neurones lead at the same time to the inhibition of the tonus or motor neurones.

The reader may object that we are now discussing interferences that do not necessarily follow from the facts so far at hand. The facts, in brief, are these. The inhibition of the tonus and the contraction of the empty stomach by stimulation of the gastric mucosa persist after isolating the stomach from the central nervous system, but the inhibition is diminished in intensity and duration after section of the splanchnic nerves, and somewhat more so after section of the vagi nerves. It has been shown that section of the vagi leaves the stomach on the whole permanently hypotonic, except during prolonged starvation, although there seems to be a gradual improvement in the efficiency of the local tonus mechanism. Is it not possible that the lessened inhibition after the vagi lesion is due to the depression of the excitability of the local afferent nerve-endings in the mucosa or depression of the local reflex center similar to the tonus depression? Our experiments do not exclude this possibility, but the results on the dogs with only the splanchnic nerves severed show conclusively that it is not the sole factor; for in these dogs there is no gastric hypotonus, and yet the inhibition from the gastric mucosa is diminished.

Another possibility has occurred to us. When the same quantity ( 25 to 50 c.c.) of acids, alkalies, or alcoholic beverages is introduced into the stomach in tonus and into a stomach in hypotonus, it seems likely that the solution will come in contact with more of the mucous membrane in the tonic than in the atonic stomach. This might result in less inhibition in the case of atonic stomach from the mere fact of stimulation of less of the afferent nervous mechanism. We have tested this possibility by introducing a greater quantity of the respective solutions in the hypotonic stomach. But if 25 c.c. of acid or beer fail to produce complete inhibition, 50 c.c. of the same liquid usually also fail. This is to be noted, however, that the depression of inhibition following splanchnic and vagi section is most marked for a week or two after these nerve lesions are made, and there is a distinct tendency in the efficiency of the local reflex pari passu with the improvement of the local tonus mechanism. 
This is probably an instance of readjustment of local reflex mechanisms to a fair degree of efficiency in the absence of central tonus and accessory central long reflexes.

The experiments on man and on normal dogs led to the conclusion that contractions of the empty stomach cannot be induced by the stimulation of the gastric mucosa-that such stimulation causes inhibition only. It was noted that one or two contractions occasionally follow immediately on the introduction of these liquids into the stomach, but it seemed probable that these contractions were due to the mechanical distension of the stomach walls rather than to the chemical or mechanical stimulation of the nerve-endings in the mucosa. These initial contractions following the introduction of acids, alkalies, or alcoholic beverages into the stomach occur more frequently in the hypotonic stomach isolated from the central nervous system. This is true even when special care is taken to introduce the substance slowly so as not to cause sudden distension of the stomach walls. I am not yet satisfied that this primary motor response is actually due to stimulation of nerveendings in the mucosa. If it is, there must be in the mucosa a few afferent nerve-endings of the excitatory type; but the afferent inhibitory nerve-endings are so much more numerous that the influence of the former group is completely submerged by the latter, except occasionally, when the stomach is hypotonic, or else local afferent nerve-endings in the mucosa are all of one type; but the type of reflex produced by this stimulation may depend in part on the tonus condition of the reflex centers (Auerbach plexus).

The local and long reflex mechanisms governing the tonus and the hunger contractions of the empty stomach demanded by the foregoing work on dogs are diagrammatically represented in Fig. 2 I. It may be noted that this diagram is not intended to represent all the afferent gastric nerve components, such as those acting in various ways on consciousness, on the vasomotor centers, etc. The adrenal glands are indicated simply as a possible factor, because conclusive data have not yet been obtained on that point. 
Rogers found that water, weak alcohol (ro per cent), weak acids ( 0.2 to 0.4 per cent $\mathrm{HCl}$ ), sugar solutions, fruit juices, etc., introduced directly into the stomach in rabbits cause temporary inhibition of the hunger contractions, but have no apparent effect on the digestion peristalsis when introduced in the filled stomach. In the guinea-pig Dr. King failed to obtain definite inhibition of the hunger contractions by chemical stimulation of the mucosa. In these experiments it is possible that the stomach was not completely empty, hence the resistance to local chemical stimulation, as Rogers and Hardt found that even in man the tonus rhythm of the fundus that is present during the digestion peristalsis is much more resistant to inhibition from chemical stimulation of the mucosa than is the very same tonus contraction in the empty stomach of the same individual.

Water and weak acids, etc., introduced directly into the empty crop of pigeons cause inhibition of the hunger contractions. In the bullfrog water, weak acids, weak alkalies, etc., inhibit temporarily both the hunger contractions and the digestion peristalsis. In the

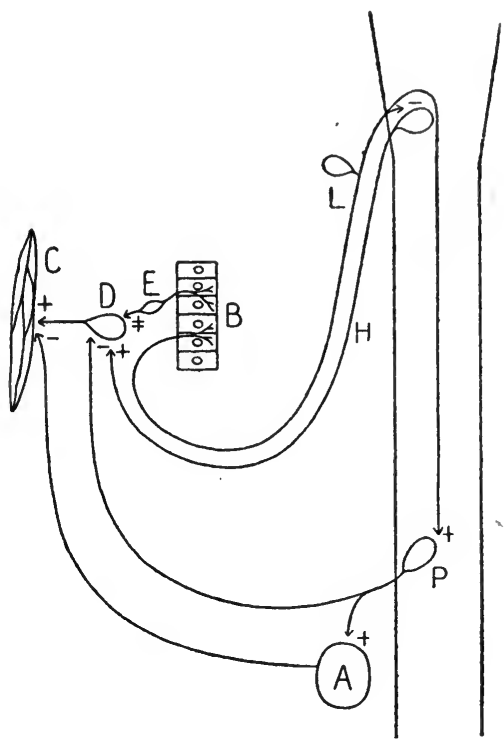

FIG. 21.-Diagram to represent the local and the long reflex mechanisms involved in the inhibition of the gastric tonus and the hunger contractions from stimulation of the gastric mucosa. $A$, adrenal gland; $B$, gastric mucosa; $C$, stomach musculature; $D$, Auerbach's plexus; $E$, local afferent neurones from the gastric mucosa to Auerbach's plexus (these neurones are predominantly inhibitory); $H$, tonus or motor neurones to the stomach via the vagi; $L$, afferent neurones in the vagi from the gastric mucosa; $P$, neurones in the splanchnic nerves; $+=$ stimulation; $-=$ inhibition. frog the inhibition from the gastric mucosa is much more marked than that produced by chemical stimulation of the nerve-endings in the mouth (Patterson). 


\section{I94 CON'TROL OF HUNGER IN HEALTH AND DISEASE}

The inhibitory reflexes from the gastric mucosa to the gastric musculature are thus present in all animals so far studied. The mechanism is probably present in all animals with a well-developed stomach. But the efficiency of the reflexes varies in different species, and in the same species or individuals they vary with the condition of the stomach (filled or empty).
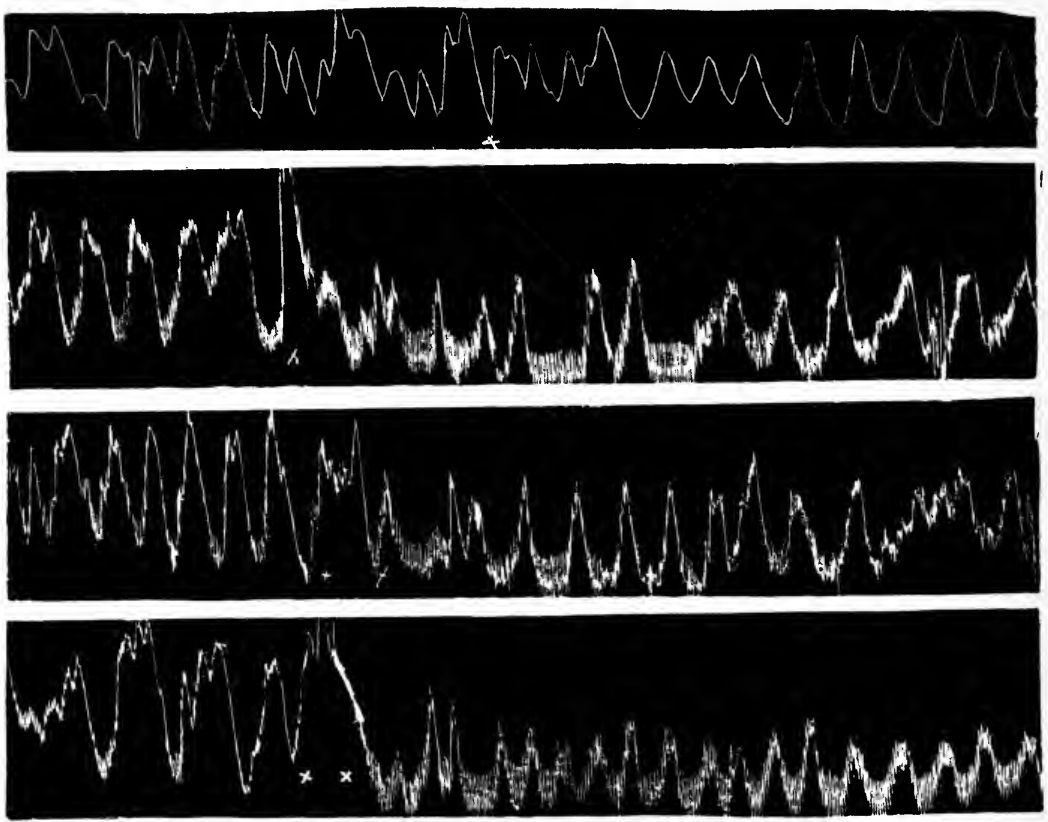

FIG. 22.- $a$, Io c.c. of 0.25 per cent hydrochloric acid put into the rabbit's stomach during normal digestion peristalsis; $b$, to c.c. of 0.25 per cent hydrochloric acid put into the stomach of a hungry rabbit; $c$, ro c.c. of water put into the stomach of a hungry rabbit; $d$, ro c.c. of ro per cent alcohol put into the stomach of a hungry rabbit. Note the inhibitory effect of these solutions on the hunger movements (Rogers).

\section{INHIBITORY REFLEXES FROM THE INTESTINAL MUCOSA TO THE EMPTY STOMACH}

We have seen that the tonus and contractions of the empty stomach are temporarily inhibited by stimulation of nerves in the mouth, in the esophagus, and in the gastric mucosa itself. Can the tonus and hunger contractions of the empty stomach be 
influenced reflexly by stimulation of the intestinal mucosa? The answer to this question might explain the diminution or abolition of hunger by the introduction of chyme into the intestine. If such reflex relations exist, it is obvious that the intestinal mucosa must be an important factor in the control of the gastric tonus and hunger mechanism.

Boldyreff reports that acids in the intestine inhibit the periodic activity of the empty stomach. The inhibition was not obtained by water or alkaline solutions. In fact, Boldyreff appears to imply that the periodic contractions of the empty stomach may be initiated by the introduction of a solution of 0.3 per cent $\mathrm{Na}_{2} \mathrm{CO}_{3}$ into the intestine. He therefore concludes that the reflex inhibition is due to an acid stimulation of nerves in the intestinal mucosa. If chemical stimulation of the intestinal mucosa induces increased intestinal tonus and contractions, we should expect the increased motility of the intestines to cause some inhibition both of the digestion peristalsis and the hunger contractions of the stomach according to the interesting theory of gastero-intestinal co-ordination recently advanced by Alvarez.

In our work we used 24 young female dogs. Intestinal fistulas were made by Abbé's lateral anastomosis in the first loop of the small intestine below the pancreas, the cephalad end being sutured into the abdominal wall and left open to the exterior. The gastric fistula was made after recovery from the first operation.

In another group of dogs a Tiery fistula was made, but no gastric fistula, the recording apparatus being introduced into the stomach through the esophagus.

In the third group the gastric fistula was made near the pyloric end of the stomach. Through this fistula a small stomach tube was passed through the pylorus into the small intestine for varying distances. This tube was kept in the gut throughout the experiment for the introduction of the liquids into the intestine. The recording balloon was passed into the stomach either through the gastric fistula or through the esophagus.

In the last group of dogs the vagi and splanchnic nerves were cut, and after recovery from the operation, gastric fistula was 
established in the antrum pylori. In all tests on this group the fluids were introduced into the intestine by means of a tube passed through the pylorus, and the stomach balloon was passed down through the esophagus.

The following solutions were introduced into the intestine in Io c.c. quantities, in most cases at body temperature: normal gastric juice (dog and man); ro per cent Witte's peptone in 0.2

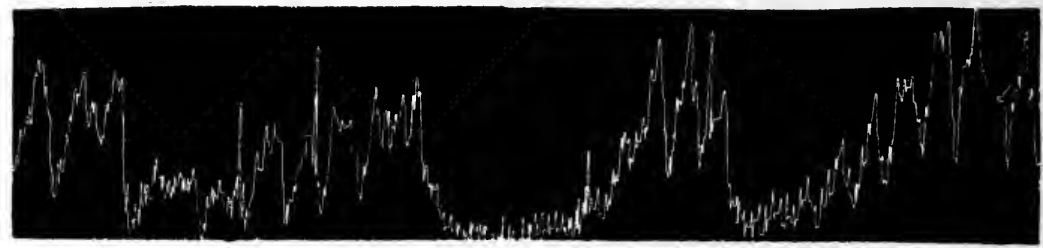

$a$ $b$

$c$

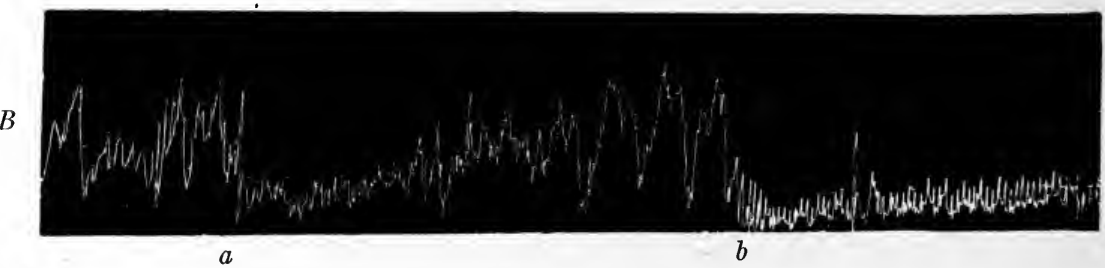

FIG. 23.-Tracings of the empty stomach of dogs. $A, a$, mechanical stimulation of intestinal mucosa (gently moving a rubber tube in the lumen); $b$, го c.c. water introduced into the intestine; $c$, Io c.c. of 0.3 per cent $\mathrm{Na}_{2} \mathrm{CO}_{3}$ introduced into the intestines; $B, a$, ro c.c. of to per cent peptone in 0.2 per cent $\mathrm{HCl}$ introduced into intestine; $b$, ro c.c. fresh milk introduced into the intestine; showing temporary inhibition of tonus and hunger contractions of the empty stomach by mechanical and chemical stimulation of the intestinal mucosa.

per cent $\mathrm{HCl}$; pepsin in 0.2 per cent $\mathrm{HCl}$; hydrochloric acid (o.I per cent to 0.5 per cent); saturated $\mathrm{H}_{2} \mathrm{CO}_{3}$ solution; neutral olive oil; fresh milk; water; mechanical stimulation of the intestinal mucosa (glass rod or rubber tube).

When the vagi and splanchnic nerves are intact all mechanical and chemical stimulations of the intestinal mucosa cause inhibition of the gastric tonus and hunger contractions. The effect of a purely mechanical stimulation (rubbing the mucosa with a glass 
rod or rubber tube) is the most transitory. In general pure gastric juice and the 0.5 per cent $\mathrm{HCl}$ cause the longest inhibition. The acid peptone solution followed these closely. The weaker acids produced inhibition of less duration. Saturated carbonic acid solution did not give quite so distinct an inhibition as the other acids. Inhibition with pure gastric juice and the acid peptone

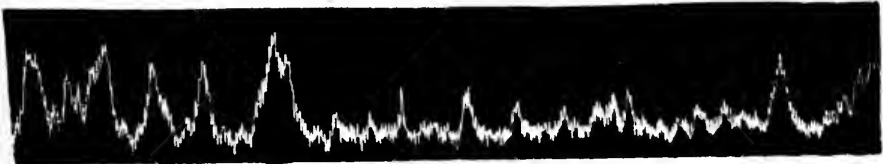

$a$

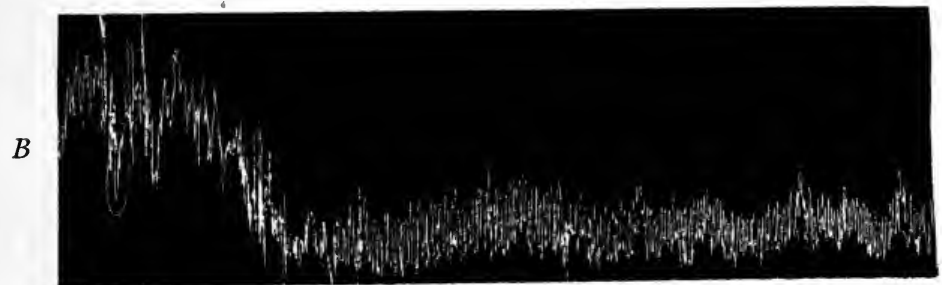

$a$

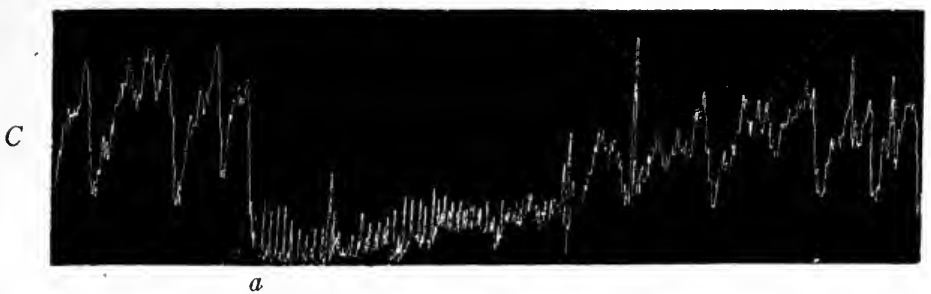

FIG. 24.-Tracing showing tonus and hunger contractions of the empty stomach of dogs. $A$, то c.c. of gastric juice introduced into small intestine at $a ; B$, ro c.c. 0.5 per cent $\mathrm{HCl}$ introduced into small intestine at $a ; C$, ro c.c. I per cent $\mathrm{Na}_{2} \mathrm{CO}_{3}$ introduced into small intestine at $a$; showing reflex inhibition of the tonus and the hunger contractions of the empty stomach by chemical stimulation of intestinal mucosa.

mixture varied in duration from 3 to 20 minutes, depending apparently largely on the condition of the animal at the time. The sodium carbonate solution caused inhibition of less duration than acid mixtures, but of longer duration than the water or the neutral 
mixtures in general. However, the longest inhibition obtained in any one experiment was produced by ro c.c. of milk in the gut. In this case the inhibition lasted 30 minutes. Ordinarily neutral solutions produced a longer inhibition than the mechanical stimulation by moving the soft rubber tube in the intestinal fistula.

In the animals with the vagi and splanchnic nerves severed the substances named above still caused reflex inhibition of the empty stomach from the intestinal mucosa, but the latent period of the inhibition was greatly prolonged, the degree of the inhibition less, and the duration of it much shorter than in the normal animals.

It is therefore clear that this inhibition of the tonus and mechanical stimulation of the intestinal mucosa involve both long or central and short or local reflex paths, a situation similar to that found in the gastric mucosa itself.

We may conclude, then, that: (I) Gastric juice, chyme, acids, alkalies, water, milk, and oil introduced into the small intestine inhibit gastric hunger contractions and gastric tonus for varying periods. (2) This inhibition is due partly to mechanical, partly to chemical, stimulation of the intestinal mucosa. The chemical stimulation produces the greatest effect. (3) This inhibition takes place primarily by the "long" or central reflex path, but "short" or 'local reflex paths in Auerbach's plexus are also involved (Elsesser).

The precise rôle of these reflexes in the control of the gastric hunger mechanism in the normal animal must be determined by further investigation. They are probably factors in the diminution or absence of hunger in cases of enteritis, intestinal obstruction, constipation, appendicitis, and gallstones. 


\section{CHAPTER XII}

THE NERVOUS CONTROL OF THE HUNGER MECHANISM (Continued)

\section{INHIBITION OF HUNGER BY SMOKING AND BY PRESSURE ON THE ABDOMEN}

It is generally held to be true that smoking shortly before a meal leads to depression of hunger and appetite. It is also a common belief that strong pressure on the abdomen ("tightening the belt") decreases or relieves the hunger sensation, at least temporarily. We are now in position to test the correctness of these beliefs by decisive experiments as regards the influence of these measures on the objective hunger contractions and the subjective hunger sensations.

Depression or inhibition of hunger by smoking is rendered probable by the fact that, at least in man, anything which stimulates the sensory nerve-endings in the mouth and in the gastric mucosa inhibits the gastric hunger contractions in direct proportion to the intensity of the stimulation. Smoking stimulates the nerveending in the mouth in varying degrees, according to the kind of tobacco used. Smoking frequently involves stimulation of the nerve-endings in the gastric mucosa owing to the swallowing of saliva containing nicotine, oils, tannic acid, and other irritating substances. Smoking may also act on the hunger mechanism in a third way, that is, through absorption of nicotine and other products of the combustion. The third possibility has not been investigated. It is well established, however, that even small quantities of nicotine in the blood leads to nausea and vomiting. Nausea and vomiting are accompanied by atony of the gastric fundus, which insures absence of hunger contractions and hunger sensations.

The effects of smoking on the gastric hunger contractions were first studied on Mr. V., our young man with the permanent gastric fistula. In his case smoking (cigars) leads invariably to inhibition 
of the hunger contractions. But Mr. V. is not a habitual smoker. It is therefore possible that the results obtained on him were simply due to the condition of nausea or disgust that smoking usually produces in the novice and hence were not applicable to persons used to smoking.

The tests were repeated on several habitual smokers. So far as smoking influences the gastric hunger contractions this influence is in the direction of inhibition. This inhibition appears to depend on the intensity of stimulation of the nerve-endings in the mouth, a cigarette or "mild" cigar causing only slight inhibition, while a "strong" cigar or pipe causes complete and prolonged inhibition, even when the gastric hunger contractions are at their maximum.

If the cigar or pipe causes very strong stimulation of the nerveendings in the mouth, the inhibition of the hunger contractions may continue from 5 to $\mathrm{I}_{5}$ minutes after the cessation of the stimulation. Thus even a brief period of smoking may suppress an entire hunger period.

The subjective sensation of hunger is diminished or abolished parallel with the gastric hunger contractions. But it seems to the authors that even a "mild" smoke diminished the sensation of hunger rather more than one might infer from the slight depression of the contractions. This is probably due to the deviation of attention, the smoking acting partly as a "counter-irritant."

Smoking inhibits the gastric hunger contractions. It is practically certain, even in the absence of direct experiments, that moderate smoking does not inhibit the gastric movements of digestion. The reason for this difference in the action of the same condition on the empty and on the filled stomach is not clear.

The experiments with constriction of the belt were made on three normal men. The tests were made with the subject standing up, sitting, and lying on the back, and at all stages of the gastric hunger contractions.

Strong contraction of the abdominal belt leads nearly always to inhibition of the gastric hunger contractions of weak or moderate strength, lasting from 5 to I $_{5}$ minutes. The inhibition may be 
partial or complete, but in either case the hunger contractions reappear despite the continued pressure of the belt. This inhibition is obtained even when the belt constriction is moderate, so that no discomfort or pain is produced.

When the gastric hunger contractions are strong (the middle of a hunger period), constriction of the belt never causes complete inhibition. But so far as the increased abdominal pressure affects the hunger contractions, the influence is in the direction of inhibition. The individual hunger contractions are weakened without suffering much change in the rate. Frequently, however, even a belt constriction that caused considerable discomfort has practically no influence on the hunger contractions, particularly if the subject is lying down.

When the gastric hunger contractions are at their maximum in rate and amplitude, as is ordinarily the case near the end of a hunger period, no amount of belt constriction seems to influence the contractions. When this stage of the hunger period is reached the hunger pangs run their normal course in the presence of even painful belt pressure.

All three subjects agreed that the belt constriction appeared to diminish or interfere with the hunger sensation to a greater extent than seemed warranted from its effect on the hunger contractions. Several factors are probably involved in this discrepancy. (I) The belt constriction distracts the attention from the hunger impulses by stimulation of nerve-endings in the viscera, especially those of the peritoneum. (2) Strong pressure on the abdomen from without appears to induce, temporarily, a condition stimulating in a feeble way the complex sensation of satiety.

According to R. Lennhoff, ${ }^{\mathbf{x}}$ hunger and appetite are appeased with a less quantity of food when the belt is constricted than when the intra-abdominal pressure is regulated solely by the tonus of the abdominal muscles. Lennhoff ascribes this to depression of hunger and appetite by the pressure of the belt. His observation is probably correct, but his explanation is erroneous. In a normal person the actual hunger contractions and hunger sensations are

${ }^{\text {r } Q u o t e d ~ i n ~ J o u r . ~ A m e r . ~ M e d . ~ A s s o c ., ~ L X ~(19 r 3), ~} 4 \mathrm{I}$. 
stopped by the first few morsels of food swallowed, while this may actually increase the appetite through stimulation of nerve-endings in the mouth and in the mucous membrane of the esophagus and stomach. This appetite sensation is gradually counteracted by the sensation complex of satiety, which depends in part on the distension of the stomach with corresponding readjustment of the tonus of the abdominal muscles. This feeling of fulness, which appears to be referred to the abdomen as a whole, is probably developed with less intake of food when the abdominal wall is mechanically prevented from relaxing owing to the pressure of the belt.

We have practically nothing but conjectures to offer in way of explanation of the mechanisms involved in these inhibitions of the gastric hunger contractions by strong pressure on the abdomen. Strong pressure on the abdomen causes temporary inhibition of the gastric hunger contractions in dogs, but the manipulation greatly disturbs them, and disturbance from any cause leads to a temporary inhibition of the empty stomach in dogs with the splanchnic nerves intact. In dogs with the splanchnic nerves sectioned on both sides strong pressure on the abdomen causes no distinct inhibition of the gastric hunger contractions.' This points to the conclusion that belt constriction causes gastric inhibition, not by direct pressure on the stomach, but by direct stimulation of inhibitory nerves, or by mechanical (or sympathetic) stimulation of the adrenal glands, and through long reflexes. Belt constriction involves stimulation of cutaneous nerve-endings, but a gentle stimulation of the tactile nerve-endings in the skin alone does not lead to this inhibition. The afferent path of the reflex must therefore involve abdominal proprioceptors. The splanchnic nerves probably constitute the. efferent path of the reflex. We do not wish to be understood as denying the existence of local inhibitory mechanisms that may be stimulated by mechanical manipulation of the abdominal organs, but our results indicate that strong belt constriction is not a sufficient stimulus for such local mechanisms. 


\section{INFLUENCE OF PHYSICAL EXERCISE AND EXTERNAL COLD ON THE HUNGER MECHANISM}

So far we have been unable to initiate or augment the gastric hunger contractions in man or experimental animals by any sensory stimulation or central nervous processes. We have seen that so far as these nervous processes affect hunger it is in the direction of inhibition. It is singular, indeed, that the inhibitory mechanisms are so readily called into play, while motor reflexes are either inaccessible or lacking, especially since the utility of some of the inhibitory reflexes are open to question at present.

From the point of view of biological adaptation we might expect the vagogastric tonus to be directly affected by voluntary muscular activity and by exposure to cold, since both conditions involve increased oxidation and consequently increased need of food.

Muscular activity may augment the gastric hunger activity by increasing the vagus tonus as well as by chemical changes in the blood. The same applies to stimulation of the cold nerve-endings of the skin. However, it is probable that if these conditions cause increase in the vagus tonus reflexly, this response is more prompt than that induced by the changes in the blood following the increase or decrease in body metabolism due to stimulation. It is generally recognized that exercise, cold climate, and cold baths increase appetite and hunger. It does not follow that these conditions actually augment the gastric hunger contractions. The increase in hunger and appetite may be only apparent, that is, may reflect a condition of increased excitability of parts of the central nervous system, so that the afferent impulses that give rise to the sensation of hunger and appetite produce a greater central effect. ' If the gastric hunger contractions are actually increased, this may be due to changes in the blood rather than to increased vagus tonus.

It is well known that exposure of the skin to cold (as by bathing in ice water) may induce contracture or "cramps" of the digestive tract. This is especially the case during the height of gastric and intestinal digestion. These cramps and contractures may be the result of circulatory disturbances or of changes in the blood rather than a direct reflex effect. Central processes are also able to induce 
contraction of the large intestine and the rectum, as shown by involuntary defecation in cases of great anxiety or fear.

\section{EXPERIMENTAL PROCEDURE}

I. Dogs.-Dogs with simple gastric fistulas were trained to run in a treadmill. When trained to run without urging or interference, records were taken of the contractions of the empty stomach so as to determine (I) whether muscular activity induces hunger contractions in the quiescent stomach, and (2) whether muscular activity augments the hunger contractions of an active stomach.

The hunger contractions of the stomach of dogs were recorded for 2 to 4 hours after a day's fast, the dogs being taken direct from the kennel without being exercised. On other days the same dogs were taken out for a 4- to 6-mile brisk walk before the 2- to 4-hour recording period.

Records of the gastric hunger contractions were taken with the dog lying quietly in the lap of an assistant. Then the body of the animal was surrounded with an ice pack, or the dog placed directly on a slab of ice. After some training the dogs do not appear much disturbed by the ice pack or slab of ice. The ice pack was applied with the stomach quiescent as well as in hunger activity.

All of these procedures were used on normal dogs and on dogs with the splanchnic nerves sectioned on both sides, in order to have the tonus fibers of the vagi unopposed by the splanchnic inhibitory influence.

2. Man.-The tests were made on the author, on Mr. V. (the gastric fistula case), and on three assistants (J. H. L., S. J. O., A. M. P.).

Records were taken of the gastric hunger and tonus contraction with the man standing or walking or running in situ. Tests were also made after muscular exercise (playing tennis, walking 6 to 2 miles).

The influence of exposure of the body to cold on the gastric hunger mechanism was tested in the following way: (I) While records of the gastric tonus and hunger contractions were being taken, the man, stripped of his clothes, was subjected to cold or warm showers for varying periods. The cold showers were at times 
sufficiently cold or prolonged to cause intense shivering. (2) The man, stripped of his clothes in a cold room, was covered up on a couch so as to feel comfortably warm. At the desired moment in the gastric activity, that is, during a period of quiescence or in the midst of a period of hunger contractions, the covers were removed and the cold air of the room set in motion by a fan placed close to the person. This brought on shivering in a few minutes. (3) The man arose at 7:00 A.M. and, without the usual cold bath and breakfast, proceeded to the laboratory, and records of the gastric tonus and hunger contractions were taken from 8:00 A.M. to I 2:00 M. These served as controls. On the other days the man arose at 6:00 A.M., took a cold bath (this was prolonged until the discomfort became very severe), followed by a brisk walk, when records were taken from 8:00 A.M. to I $2: 00 \mathrm{M}$.

\section{RESULTS ON DOGS}

I. Effects of running in treadmill.-The initial effect on gastric tonus and hunger contractions of running in the mill is always in the direction of inhibition - usually complete inhibition-and if the dog is started running in the midst of a period of gastric quiescence there is no evidence of increased gastric tonus or beginning of hunger contractions. If the dog is made to run at high speed the inhibition persists during the entire period, even if the running is kept up for one or two hours. When the dogs ran at rather high speed for an hour or more the gastric inhibition usually persisted from 20 to 40 minutes after the dog stopped running. The return of gastric tonus and hunger contractions in such cases is very gradual. But frequently when the gastric tonus finally recovered after a running period it was higher than before the dogs began to run. Thus a dog showing type I or II of hunger contractions when he started to run in the mill showed an increased tonus and type III of hunger contractions 30 minutes after he stopped running, while the running period itself was accompanied by complete gastric inhibition. If the dog runs only moderately fast in the mill the gastric tonus and hunger contractions reappear during the running 
period, or come on during the running, in case the dog is started when the empty stomach is quiescent.

These facts indicate that the carnivorous animal in pursuit of its prey must be urged on by something else than the pangs of hunger, as these are inhibited by the chase. Brisk walking or running leads also to inhibition of the digestion movements of the stomach, according to the observations of Cohn on dogs, Bender on man, and Scheunert on the horse.

2. Effects of 4-to 6-mile walk.-Eight tests (with a corresponding number of controls) on two dogs failed to show any marked effect of a 4- to 6-mile walk on the gastric hunger contractions either in the way of increase or decrease, the records being taken during the two hours following the walk. These walks certainly caused no depression of the dog's hunger contractions. But the dog that showed type II contractions in the control usually showed type II contractions after the walk, with no definite increase either in rate or intensity. This should be noted, however, that after these walks both dogs showed greater restlessness than when taken from the kennels directly to the laboratory. They were not so easily quieted in the lap of the assistant. This rather restless condition of the dogs may have counteracted any augmentation of gastric hunger contractions due to the walk, as restlessness from any cause tends in the dog to inhibit the hunger contractions.

3. Effect of intense stimulation of the cutaneous nerve-endings for the sensation of cold.-When a dog is lying quietly and comfortably in the lap of an assistant, surrounding the dog with an ice pack or placing him directly on a slab of ice leads to struggling and restlessness. After a number of repetitions of these procedures most dogs become so accustomed to it that they pay little or no attention to the change and show no restlessness or struggling. If the dog is disturbed or struggles when placed on the slab of ice or surrounded by an ice pack there always follows a temporary inhibition of gastric tonus and hunger contractions. But this does not indicate the initial or primary effect of stimulation of the cutaneous nerveendings for cold, because the same type of inhibition is induced by restlessness or struggle for any cause. After the dog is trained to 
these procedures strong stimulation of the cutaneous nerve-endings for cold by the ice pack, by placing the dog on a slab of ice, or by turning on an electric fan in a cold room after uncovering the dog, has no immediate effect on the gastric tonus and hunger contractions. There is usually an increase in the intra-abdominal pressure owing to the increased tonus of the abdominal muscles. If the ice pack is applied during a period of gastric quiescence there is no immediate increase in gastric tonus or initiation of the hunger contractions, even though the dog starts to shiver violently in a few minutes. If the ice pack is applied during the hunger contractions, these contractions do not change appreciably either in rate or strength, at least for some time. This is true even when the dog shivers considerably. It would thus seem that the vagus centers governing the gastric tonus are not directly affected by even very strong stimulation of the cutaneous nerve-endings for cold.

In several instances the continued application of the ice pack ( 30 to 40 minutes), and in consequence continued shivering, led to a gradually increased gastric tonus and the appearance of type III hunger contractions. These may be due to changes in the blood as a result of increased oxidation, or they may appear from causes not connected with the stimulation of the cold nerve-endings. Such change in the hunger contractions is not infrequent in dogs, even when they are lying undisturbed and comfortable in the lap of an assistant.

It is conceivable that the stimulation of the cold nerve-endings in the skin does influence the vagogastric tonus centers, but the stimulation acts equally on the gastric inhibitory mechanism via the splanchnic nerves, so that the net result on the empty stomach is nil. This possibility is cleared up by the test on dogs with section of both splanchnic nerves. Tests were made on two dogs on which the operation had been performed. The results were practically identical with those on normal dogs. The ice pack neither decreased nor increased the gastric hunger contractions. It is therefore clear that the nervous impulses that give rise to the sensation of cold and induce increased neuromuscular tonus in general have no direct action on the vagogastric tonus centers. 

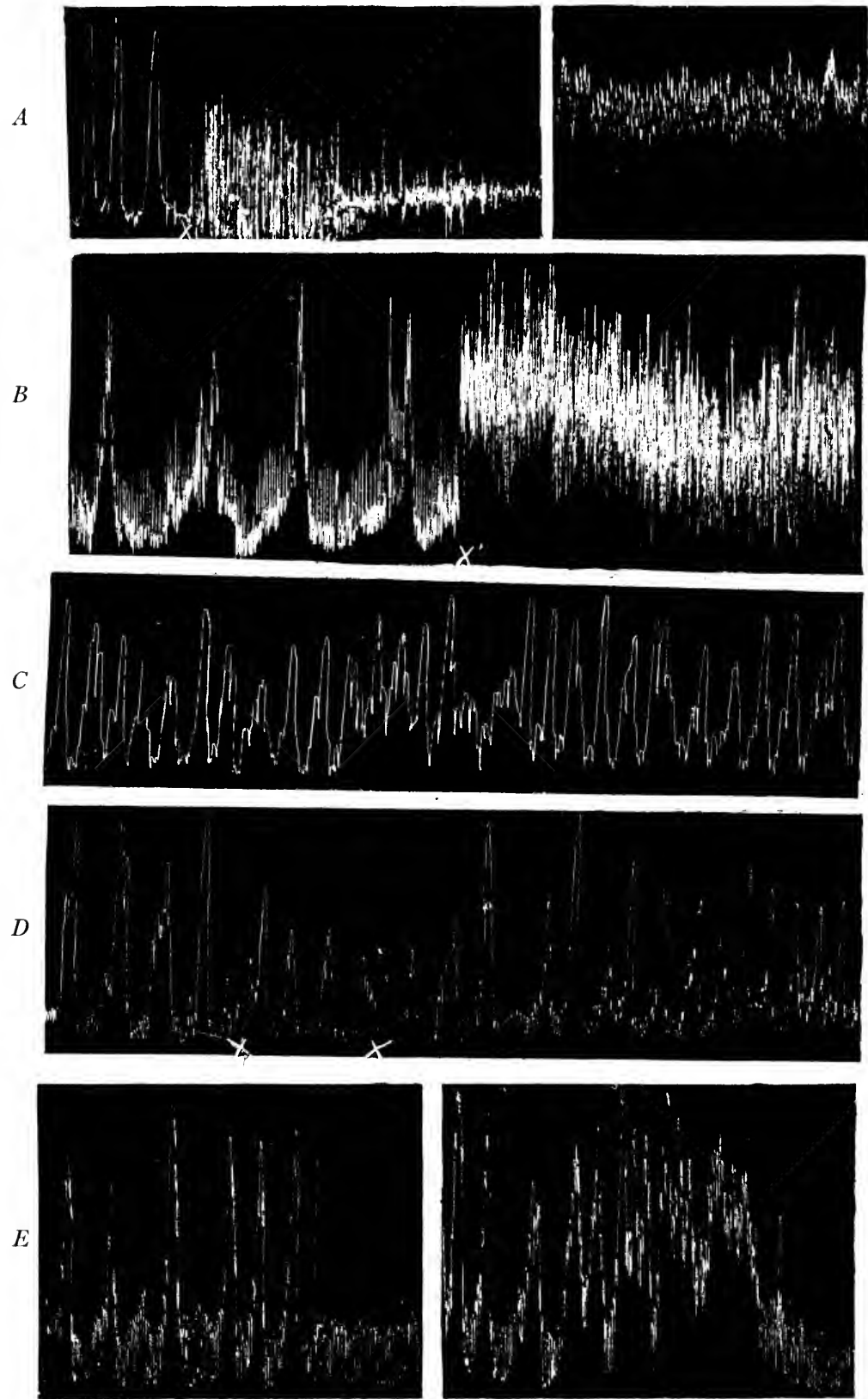

FIG. 25 
FIG. 25. $-A$, tracing from the empty stomach of a dog. I. Dog standing in treadmill, stomach showing type I hunger contractions. At $x$, the dog begins to run in the mill with the result that gastric hunger contractions are promptly inhibited. The running was kept up for 60 minutes. II. Record from stomach of same dog 45 minutes after he ceased running, showing increased gastric tonus. $B$, tracing from the empty stomach of man (A. J. C.) in standing position; beginning of a hunger period. At $x$, the man began running, with the result that the hunger contractions wer $\mathrm{e}$ promptly inhibited. $C$, tracing from the empty stomach of dog with section of splanchnic nerves on both sides. At $x$ the dog is surrounded by an ice pack. $D$, tracing from the empty stomach of man (A. J. C.) in the midst of a period of hunger contractions. The man was stripped and covered up with blankets in a cold room $\left(20^{\circ} \mathrm{C}\right.$.). At $x$ the covers were removed and a fan close to the man started. Shivering began at $x^{\prime}$; showing a temporary but partial inhibition of the hunger contractions. $E$, records of culminations of periods of gastric hunger contractions of A. J. C. I. The ordinary type of ending of the hunger periods without tetanus. II. Hunger period ending in incomplete tetanus three hours after intense stimulation of the cold nerve-endings (bath at $10^{\circ} \mathrm{C}$. for 15 minutes). 


\section{RESULTS ON MAN}

I. Direct effect of muscular exercise.-Standing or walking in situ has no effect on the gastric tonus or the hunger contractions. But running in situ promptly inhibits the hunger contractions. The degree and duration of the inhibition are on the whole directly proportional to the speed of the running. In some cases walking seemed to prolong a hunger period without changing the rate or intensity of the individual contractions. In no case did walking or running induce hunger contractions in the quiescent stomach. The results on man are thus identical with the results on dogs. In both species rapid running is accompanied by inhibition of the gastric tonus and hunger contractions. In the case of the dog running in the treadmill, one cannot be sure that the exercise is strictly voluntary and enjoyable. The inhibition may therefore be due to certain emotional states (anxiety, discomfort, mild anger, or fear). This possibility is eliminated by the tests on man. In the men the conditions of the emotions when running in place were not different from that when standing or walking in place. In no case was the running carried to the point of respiratory, cardiac, or muscular distress.

2. After-effects of muscular exercise.-Moderate exercise in the form of playing tennis or walking 4 to 8 miles was taken in the afternoon. No supper was taken, and the motor condition of the empty stomach was recorded from 8:00 P.M. to I 2:00 midnight. The tracings obtained on the days specific exercise was taken show on the whole greater gastric hunger activity than the controls. The periods of quiescence become shorter. This tends to make the gastric hunger contractions more or less continuous, and there appears to be some increase in the rate of the contractions. A typical experiment (S. J. O.) may be cited in the way of illustration.

Record of control day.-Lunch I:30 P.M. No special exercise. No supper. Period of observation 8:00 P.M to I 2 midnight.

8:00 to I0:00 P.M. Stomach practically quiescent.

I0:00 to I0:40. Strong hunger contractions, ending in tetanus.

IO: 40 to II: 35 . Stomach quiescent.

II $: 35$ to $12: 05$.

Moderate hunger contractions ending in tetanus. 
Record of cxercise day.-Lunch I:30 P.M. No supper. 'Tennis 4:00 to 5:00 P.M.; walking 6:00 to 7:00 P.M. Period of observation 8:00 P.M. to I 2 midnight.

8: I5 to 9:50 P.M. Practically continuous hunger contraction ending in strong tetánus.

9:50 to ro: 20 . Stomach quiescent.

IO: 20 to II $: 40$.

Strong hunger contractions ending in tetanus.

(Control day, 70 minutes; exercise day, I90 minutes.) Total duration of hunger periods from 8:00 P.M. to I 2 midnight.

In some instances there was no marked difference between records of the control and the exercise days. This is to be expected, since the activity of the gastric hunger mechanism depends in part on factors not understood or controlled. Exercise that brings on a degree of fatigue bordering on exhaustion seems to depress the gastric hunger mechanism. But our experiments on this point are as yet too few to permit a final conclusion.

3. Direct effect of stimulation of the cold nerve-endings of the skin.The immediate effect of stimulation of the cold nerve-endings of the skin by ice pack, alcohol bath, cold shower bath, or cooled air is inhibition of the gastric tonus and hunger contractions, and the degree of inhibition is proportional to the intensity of the stimulation. In no instance did we observe an initial increase in gastric tonus and hunger contractions. When the stimulation is continued the inhibitory effects gradually diminish, even though the man shivers intensely from the cold. In this way the gastric hunger contractions may return to their normal rate, intensity, and regularity, while the man is shivering and jerking like a dog in mild parathyroid tetany. It may be noted in this connection that mild and in some instances fairly severe parathyroid tetany in dogs does not appreciably influence the gastric hunger contractions.

Intense stimulation of the heat nerve-endings of the skin (hot shower) produces practically the same initial inhibition as the corresponding stimulation of the cold nerve-endings.

While it is true that on prolonged stimulation of the cold nerveendings of the skin, during a period of gastric hunger contractions, the inhibitory effects gradually disappear, so that the contractions 
reappear in their normal intensity, these contractions are always felt as weaker than the normal, or may not be felt at all. Evidently the intense sensation of cold dominates consciousness to the exclusion of the gastric hunger pangs.

It is well known that strong stimulation of the cold nerveendings of the skin causes a reflex increase of tonus of the urinary bladder. In several instances we started these stomach tests on the men at a time when their bladders were known to contain 50 to 200 c.c. of urine. This permitted us to compare the reflex effect of cold on the stomach and bladder tonus without a balloon in the bladder. When the cold stimulation began during a period of gastric quiescence and was continued long enough to induce intense shivering, a strong desire to micturate soon developed while there was no evidence of increased gastric tonus. Prolonged cold stimulation may produce so great a tonus of the bladder that micturition cannot be inhibited voluntarily. The tonus centers of the urinary bladder are, the vagogastric tonus centers are not, directly influenced by cold stimulation of the skin.

When the cold nerve-endings of the skin are stimulated; as described above, during a period of quiescence of the empty stomach, the stomach remains quiescent. If there is any change in the gastric tonus it is in the direction of inhibition. Nevertheless, this cold stimulation, if not sufficiently intense to be painful, seemed to induce a "sensation of emptiness" in the abdominal region, a sensation that seemed to be associated with appetite and desire for food. We record this with some hesitation, for this sensation of emptiness may be purely subjective (auto-suggestion). It may also be due to the increased tonus of the abdominal muscles. In any event this sensation is clearly different from the hunger pangs.

4. After-effect of the stimulation of the cold nerve-endings of the skin.-All of the tests in this group were made on one man (A. J. C.). A prolonged cold bath, from 6:00 to 7:00 A.M., followed by a brisk walk, nearly always resulted in increased hunger activity of the stomach as recorded for the period 8:00 A.M. to I2:00 M. The temperature of the water varied from $5^{\circ} \mathrm{C}$. to $15^{\circ} \mathrm{C}$. The subject remained in the water as long as was deemed safe (Iо to 20 minutes) despite discomfort and pain. Water at this temperature soon brings 
on shivering, contracture, and at times severe headache, and it requires much vigorous exercise to restore the feeling of warmth. Rubbing the skin (rough towel) seems to be of little avail. A typical experiment may be cited in illustration.

Control record. -No bath or breakfast. Observation period 8:00 A.M. to I $2: 00 \mathrm{M}$.

8:50 to I0:00 A.M. 26 fairly strong hunger contractions; no tetanus. II : 00 to II $: 45$. 22 fairly strong hunger contractions; no tetanus. Gastric tonus on the average $5 \mathrm{~cm}$. bromoform.

Test period. -6:00 to 6:I 5 A.M., cold bath (temperature of water $10^{\circ} \mathrm{C}$.). No breakfast. Observation period 8:00 A.M. to I 2:00 M.

8:00 to 9:00 A.M. 32 strong contractions; no tetanus.

$9: 45$ to $10: 25$. 23 fairly strong contractions; no tetanus.

II:I 5 to II:45. I9 strong contractions ending in tetanus.

Gastric tonus on the average $8 \mathrm{~cm}$. bromoform.

Control period. -48 hunger pangs; no tetanus.

Test period.-74 hunger pangs; tetanus.

Under ordinary conditions the periods of gastric hunger contractions of the author do not end in tetanus, but the hunger tetanus appears after 3 to 4 days' complete starvation. Intense stimulation of the cold nerve-endings for 15 to 30 minutes thus seems to bring about a condition similar to prolonged starvation. This is in harmony with the observation of Lusk that such stimulation quickly renders the liver free from glycogen. This effect of cold on the gastric hunger mechanism is obviously an indirect one, or brought about through changes in the blood, and is not a direct reflex from the skin.

Lusk has shown that intense cold leads to quicker and more complete oxidation of the body glycogen than prolonged starvation. And it is interesting to note that the same stimulus causes not only an increase in the gastric hunger contractions, but also an even greater increase in the subjective hunger and appetite sensations, probably owing to an increased excitability of the central nervous system. The increased desire to eat after a cold bath, in the case of the healthy individual, is a universal experience. I have investigated this matter in the case of young children, with whom habit or intelligence cannot be assigned as the cause for seeking food 
after a cold bath. It was found that young children react in the same way as adults.

While these observations include only two species (man, dog), it does not seem likely that the gastric vagotonus mechanism will have different reflex associations in other animals. But this opinion should not stand in the way of actual investigation of the condition in other vertebrates as well as in the invertebrates. In man and in dogs the situation appears to be this: The vagus motor nuclei in the medulla control, in part, the tonus and hunger contractions of the stomach. The tonus of the vagus nuclei, in turn, are controlled by the condition of the blood rather than by afferent nervous impulses, unless sensory impulses from the stomach musculature itself play such a rôle. This possibility is now under investigation.

III. AFFERENT OR SENSORY PATHS OF THE HUNGER COMPLEX AND THE QUESTION OF THE CEREBRAL "HUNGER CENTER"

I. Rôle of the vagi.-The vagi nerves are the main, if not the only, afferent pathway for the gastric hunger impulses, although Luciani assumes that some of the hunger impulses from the stomach are carried by the sympathetic (splanchnic) fibers. If the contraction of the small intestine contributes to the hunger sensation, the afferent hunger impulses may involve sympathetic and spinal nerves, but all sensory conduction from the stomach appears to be confined to the vagi, because no central reflexes of any kind can be evoked by stimulation of the stomach after section of all the vagi fibers to that organ (Miller). We need not here refer to the physiologists who have argued against the vagi nerves (and in favor of the splanchnics) as being concerned in hunger on the basis that animals will continue to eat after section of these nerves or after excision of the stomach, as these objections have already been considered and refuted.

2. The primary hunger center is therefore the sensory nuclei of the vagi nerves in the medulla (fasciculus solitarius). Some of the more direct hunger reflexes (such as salivation, vasomotor fluctuations, etc.) may be carried out via these medullary centers alone. Luciani assumes also spinal hunger centers analogous to 
these sensory vagi nuclei. There is no evidence that the processes of conscious hunger sensation can take place in the medullary nuclei.

3. Rôle of the optic thalami and the mid-brain.-The important facts in this connection are the hunger behavior of decerebrated animals (acephalic infants, dogs, pigeons, frogs). This hunger behavior has already been ascribed. 'These animals minus the cerebral hemisphere, but with the thalamic region of the brain intact, exhibit practically all the hunger behavior of normal animals, except the intelligent search for and ingestion of the food. But even this statement requires limitation, for, according to Ewald, ${ }^{1}$ decerebrated pigeons and frogs will finally eat sponțaneously if kept in good condition for a sufficient time (months) after the operation.

Rogers has recently made the important observation that the hunger behavior of the decerebrated pigeon is completely abolished on removal of the optic thalami. It is thus clear that this region contains important nuclei for the elaboration of the bodily responses to the hunger impulses from the stomach. Whether or not the processes of conscious hunger sensations are elaborated in the thalamus cannot be determined on experimental animals. L. R. Müller assumes that conscious hunger sensations are evoked in the mid-brain. We have seen that hunger is essentially pain, and some neurologists take the position that the sensation of pain is a thalamic rather than a cortical function. This view is supported by the extensive studies of Head and Holmes on the change in the pain sense in persons with thalamic lesions and intact cortex. The frequent occurrence of excessive hunger or polyphagia in persons with tumors of the pineal glands have by some (Schüller) been interpreted as due to a pressure stimulation of subcortical hunger centers. Whether the thalamic processes caused by the gastric hunger contractions are conscious or merely subconscious reflexes, or whether the nuclei concerned with these processes are identical with those involved in pain sensation, it is clear that the thalamus

${ }^{x}$ Quoted from A. L. Gillespie, The Natural History of Digestion, New York, I898, 286. 
is a very important reflex and relay station for the afferent hunger impulses.

4. Cortical hunger centers.-Concerning these practically nothing is known. There can be little doubt that conscious hunger

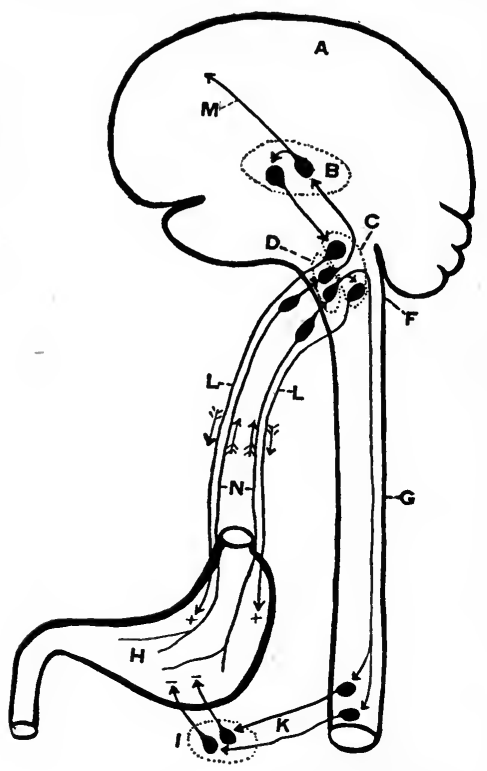

FIG. 26.-Diagram of the nervous mechanism of the hunger sense. $A$, cerebrum. $B$, optic thalami. $C$, motor nuclei of the vagi nerves. $D$, sensory nuclei of the vagi nerves. $F$, medulla oblongata. $G$, spinal cord. $H$, stomach. $I$, visceral sympathetic ganglia. $K$, splanchnic nerves. $L$, motor fibers to the stomach. $M$, sensory paths (hunger) to cerebrum (hypothetical). $N$, sensory fibers from stomach in the vagi. + indicates motor, - indicates inhibitory effects, $\rightarrow$ indicates direction of nervous conduction.

cal investigator to correct the guesses and fill up the gaps, as very little can be done with these problems on animals below man, at least with the methods so far available to the physiologist.

involves in some way cortical processes, and one might expect the part of the cortex involved would be contiguous with that for the gustatory sense, the latter being placed in the hipochampal gyrus by the majority of neurologists and psychiatrists. Roux assumes that the cortical hunger center is in the Rolandic area, thus supporting Bechterew, who locates the conscious taste processes in the region of the Rolandic area which innervates the muscles of mastication and deglutition.

To recapitulate: In the afferent phase of the hunger complex the facts clearly established are the rôle of the vagi, and the sensory vagi nuclei in the medulla, and the great importance of the thalamus. The cortical factors in hunger are unknown, and the same applies to the detailed rôles of the subcortical hunger centers both in health and in disease. This field of the physiology of hunger is therefore mainly "gaps and guesses." It remains for the clini- 


\section{CHAPTER XIII}

\section{THE CHEMICAL CONTROL OF THE HUNGER MECHANISM}

\section{ANALYSIS OF THE PROBLEM}

The reader will recall from the review of the literature on hunger and appetite in chap. ii that most of the authors assume a chemical control of the hunger mechanism in the sense that starvation changes of the blood stimulate the conscious hunger center in the brain, the specific sensory nerves in the stomach, or sensory nerves in all the tissues. We know now that the hunger sensation is caused by strong contractions of the empty or nearly empty stomach. This fact modifies but does not eliminate the question of chemical control of the hunger mechanism. We must now determine whether starvation changes in the blood influence the motor side of the mechanism, while the earlier authors considered the influence of the blood only on the sensory side of the hunger apparatus.

Chemical changes in the blood may act in a positive way either on the vagi tonus centers in the brain or directly on the stomach motor tissues. The problem of chemical control of hunger is thus resolved into three main queries, namely: (I) Do chemical stimuli in the blood cause the increased gastric tonus of hunger? (2) Do chemical stimuli in the blood initiate the individual gastric hunger contractions? (3) Do chemical stimuli control the grouping of the hunger contractions into hunger periods, separated by periods of relative gastric quiescence, in species showing this grouping?

We must also consider the possibility of chemical blood changes that may depress or inhibit the hunger contractions.

The gastric hunger contractions are inhibited by mechanical and chemical stimulation of the nerve-endings in the mucous membrane of the mouth, the esophagus, and the stomach. This insures inhibition of the hunger contractions during mastication and gastric digestion. The gastric hunger mechanism receives motor or tonic innervation via the vagi, and the central connections of this tonus 
innervation appear to be practically isolated from all normal reflexes, while the inhibitory mechanism via the splanchnic nerves is very readily called into activity reflexly. The foregoing facts appear to have only two alternative explanations, viz: (I) The gastric hunger contractions are due to a specific automatism (vagi centers and stomach) primarily independent of afferent impulses as well as the conditions of the blood. Such an automatism would, of course, vary with the physiological condition of the automatic tissues; but if this is the mechanism we cannot speak of any physiological nervous control of the hunger apparatus, except in the way of inhibition. (2) The vagi and the gastric mechanism concerned in the genesis of the hunger contractions may be influenced in a positive way by physiological changes in the blood. If this is the case, we might expect such changes in the blood to be specially evident in the normal animal when starving.

Some of the facts already discussed seem to show that both of the factors named above are to be reckoned with. In man and dog the gastric hunger contractions usually appear as soon as the stomach is empty of food, that is, before intestinal digestion and absorption of the meal are completed. Under these conditions the initiation of the hunger contractions must be due to a primary automatism not opposed by inhibitory reflexes rather than to any changes in the blood such as are presumably involved in starvation, for there is surely no auto-digestion of the body tissues or lack of pabulum in the body fluids while normal intestinal digestion and absorption are still in progress. In dogs with Pavlov stomach pouches we may also have hunger contractions in the main stomach while the Pavlov stomach is quiescent, or vice versa. On the other hand, excessive hemorrhage, prolonged starvation, and pancreatic diabetes, which is a type of starvation, lead to increased activity of the hunger mechanism, at least up to the point where the stomach becomes directly involved in the general debility and cachexia. The increased vigor of the hunger apparatus in normal individuals as an after-effect of the greatly accelerated metabolism caused by physical exertion and cold, is a bit of evidence pointing in the same direction. 
This augmentation of the hunger contractions in starvation may be due to (I) the appearance of substances in the blood stimulating the central tonus mechanism or the peripheral hunger apparatus; (2) the absence or diminution of inhibitory substances in the blood; (3) the absence or depression of inhibitory reflexes; (4) starvation changes in the tissues directly concerned in the hunger contraction.

If it is due to the presence of stimulating substances in the blood, it would seem that transfusion of the blood of starving animals into normal animals ought to augment the activity of the hunger mechanism, at least temporarily. This is actually the case.

\section{ACTION OF BLOOD OF STARVING ANIMALS ON THE GASTRIC} HUNGER MECHANISM OF NORMAL ANIMALS

Direct transfusion from the starved donor to the normal recipient by direct union of blood vessels is not feasible, because if this is done under general anesthesia, the anesthetic itself depresses the stomach, and if it is done with aid of local anesthesia only the recipient is so disturbed that the stomach is inhibited reflexly. But we found that good-natured and gentle dogs used to our routine of recording the gastric hunger contractions were practically not disturbed at all by the puncture of the saphenous vein with a sharp needle and the injection of 20 to 50 c.c. fresh-drawn and defibrinated blood. This technique was therefore adhered to. In the preliminary training of these dogs the animal's legs were handled in various ways (shaved, injection of salt solution, etc.), so that the animal finally paid little or no attention to the handling of the leg or the insertion of the needle into the vein. In some cases we decreased the sensitivity of the skin over the saphenous vein by the application of carbolated vaseline.

The intravenous injection of 20 to 50 c.c. of fresh defibrinated blood from starving dogs into normal dogs increases the gastric tonus and hunger contractions of the latter, if their stomachs are empty and if moderate tonus and hunger contractions are in evidence in the recipient at the time of the injection of the blood. If the stomach of the recipient, although empty of food, is atonic and 
hunger contractions are completely absent at the time of the injections, the blood from starving animals has practically no action on the stomach. The stimulating action of this blood on the stomach already in moderate tonus and hunger contractions lasts from to to 30 minutes, depending on the quantity of starved blood injected.

The foregoing conclusion is based on 25 experiments on 4 gastric-fistula dogs. The blood for the transfusion was drawn from animals after 5 to $\mathrm{I} 2$ days of starvation.
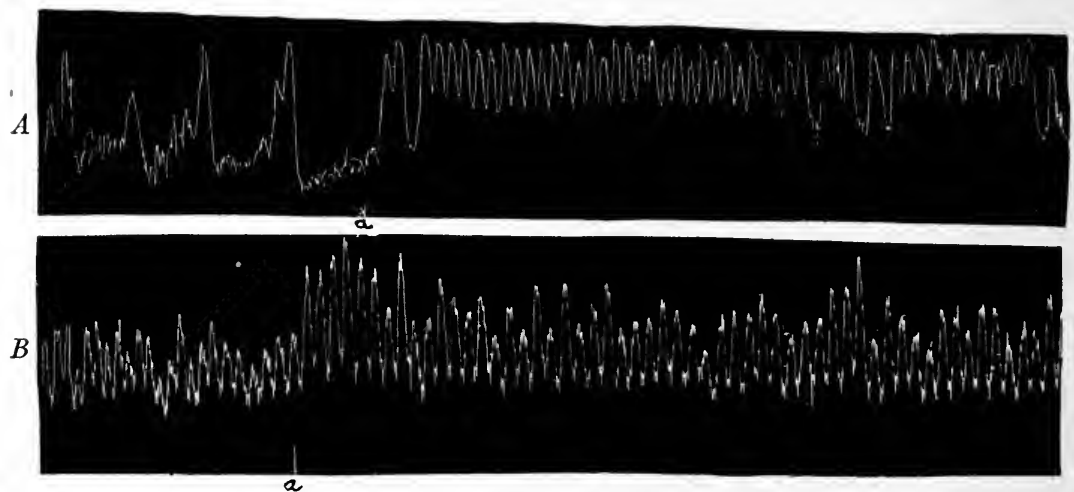

FIG. 27. -Tracings from the empty stomach of dogs. $A$, at an intravenous injection of 35 c.c. blood drawn from a dog on the eighth day of starvation; showing stimulation of the gastric hunger apparatus, in the change from type I to type III hunger contractions (hunger tetanus). $B$, at an intravenous injection of 20 c.c. of blood from a dog in pancreatic diabetes; showing stimulation of the gastric hunger mechanism (Luckhardt and Carlson).

The failure of starved blood to induce tonus and hunger contractions in atonic and quiescent stomachs is probably due to the fact that by the present method of transfusion it is not possible to introduce enough starved blood to overcome the inhibitory factors responsible for the atonic and quiescent condition.

\section{EFFECTS OF BLOOD FROM DIABETIC ANIMALS}

Under the technique described above, 20 to 50 c.c. of blood from animals in pancreatic diabetes and showing the typical diabetic polyphagia were transfused into normal animals. The results 
were practically identical with those from the blood of starving animals, that is, a temporary stimulation of the gastric hunger mechanism.

From 20 to 50 c.c. of blood from normal dogs or from dogs whose digestion was at its height were transfused into dogs while their gastric tonus and hunger contractions were being registered. In the majority of these experiments the transfusion had no effect at all on the motor condition of the empty stomach. In a few cases it acted as a very slight and transient stimulus, but in no instance did the blood from normal animals produce the marked effects obtained from the blood of starving and of diabetic animals. Hence we conclude that the latter results are due to something in the blood of starving and of diabetic animals not present, or present in less concentration, in the blood of normal animals. It is evidently not due to the transfusion of the above-named quantities of defibrinated blood as such, although defibrinated blood contains a substance which induces contraction in vascular and intestinal muscle. The intravenous injections of 20 to 50 c.c. of 0.9 per cent $\mathrm{NaCl}$ is also without effect on the hunger mechanism.

It is well known that intravenous injections of considerable quantities of fresh defibrinated blood may cause temporary vasomotor and cardiac disturbances. Lowering of the arterial blood pressure is usually a feature of these disturbances. Is vasodilation a factor in the marked results produced by blood from starving and diabetic animals? The following control tests were made: A mixture of I per cent peptone in 0.9 per cent $\mathrm{NaCl}$ was injected intravenously, and amyl nitrite was administered by inhalation. If sufficient peptone or amyl nitrite is given to affect the gastric tonus and hunger contractions, this effect is always in the direction of inhibition and paralysis. It is not clear, however, that this inhibition is due solely to the vasodilation, but the experiments show that a moderate general vasodilation does not necessarily lead to stimulation of the gastric hunger apparatus.

As a preliminary step in the analysis of the stimulation of the gastric hunger mechanism by starved and diabetic blood, we have tested the action of acetone and oxybutyric acid on the gastric 
hunger contractions. It is well known that prolonged starvation as well as diabetes leads to acidosis, although there is practically no acidosis in pancreatic diabetes in dogs (Mariott). It seemed possible that the acetone bodies might be the stimulating factors in the starvation and the diabetic blood. The action of the acetone bodies dissolved in Ringer's solution was tested on a number of animals with uniformly negative results. That is to say, the acetone bodies in concentrations that affect the gastric hunger apparatus at all cause inhibition and depression. No indication of any primary or secondary stimulation by the acetone bodies could be secured. It is therefore clear that the stimulating action of starvation and diabetic blood on the hunger mechanism is not due, at least not directly, to the acetone bodies.

\section{EFFECT OF HEMORRHAGE ON THE GASTRIC HUNGER MECHANISMM}

Some of the blood conditions of starvation may be produced temporarily by excessive hemorrhage. It is recognized, of course, that hemorrhage also introduces factors not present, at least in moderate starvation, such as the temporary diminution of hemoglobin. Nevertheless, the results of two series of experiments with the effects of excessive hemorrhage are so striking and conclusive that they are reported, even though we have not worked out their interpretation. The results are most conveniently stated by the following brief protocols:

\section{Dog I. Weight $6.0 \mathrm{~kg}$.}

Oct. 20. Types II and III gastric hunger contractions.

" $2 \mathrm{I}$. Type I contractions. Gastric tonus $=3 \mathrm{~cm}$. chloroform.

"22. "I "

"23. " I "

" 24 . " I "

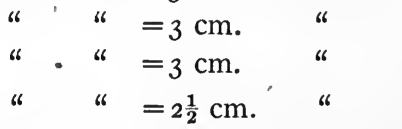

" 27. 9:I2 A.M., light ether anesthesia; I46 c.c. blood drawn from carotid artery at 9:30 A.M. Recording of the gastric hunger contractions began Io:08 A.M. At this time the stomach was atonic and quiescent. A gradual return of gastric tonus appeared at Io:30 A.M. At II :०० A.M. the gastric tonus was $5 \mathrm{~cm}$. chloroform with vigorous type III hunger contractions, and this condition persisted till the end of the experiment at $\mathrm{I} 2: 30$ P.M. 
Oct. 28. Type I contractions. Gastric tonus $=2 \frac{1}{2} \mathrm{~cm}$. chloroform.

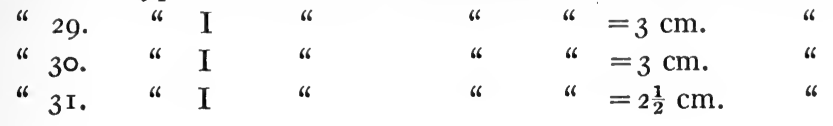

\section{Control Experiment on Dog I}

November I8, Ether Anesthesia for 20 Minutes

Nov. I8. Type I contractions (very feeble). Gastric tonus $2 \mathrm{~cm}$. chloroform.

" I9. "I contractions (feeble). Gastric tonus $2 \mathrm{~cm}$. chloroform.

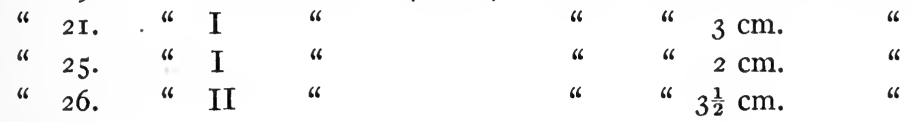

Dog II. Weight $6.7 \mathrm{~kg}$.

Oct. 30. Type I hunger contractions. Gastric tonus $2 \mathrm{~cm}$. chloroform.
" 3 I.
"
"
" " $2 \mathrm{~cm}$.
“

Nov. 3. " I and II hunger contractions. Gastric tonus $3 \mathrm{~cm}$. chloroform.
" 4. " I hunger contractions.
5. " I " " " " $2 \mathrm{~cm}$.

“ 6. 9: IO A.M., I69 c.c. blood withdrawn from carotid artery under light ether anesthesia. Record of gastric contractions began at 9:45 A.M. At this time the stomach was quiescent with feeble tonus. At Io:०0 A.M. the gastric tonus began to increase. At Io:30 A.M. the gastric tonus was $9 \mathrm{~cm}$. chloroform with type III vigorous hunger contractions. This condition persisted till the end of the experiment at II : 30 A.M.

Nov. 7. Types II and III contractions. Gastric tonus $2 \frac{1}{2}$ to $3 \mathrm{~cm}$. chloroform.
"II.
" II and III
" I2. Type I
" " 3 to $7 \mathrm{~cm}$. "
" $\quad 2 \frac{1}{2} \mathrm{~cm}$.

\section{CONTROL Experiment on Dog II}

November I8, Ether Anesthesia for 20 Minutes

Nov. I8. Types I and III contractions. Gastric tonus I to $4 \mathrm{~cm}$. chloroform.

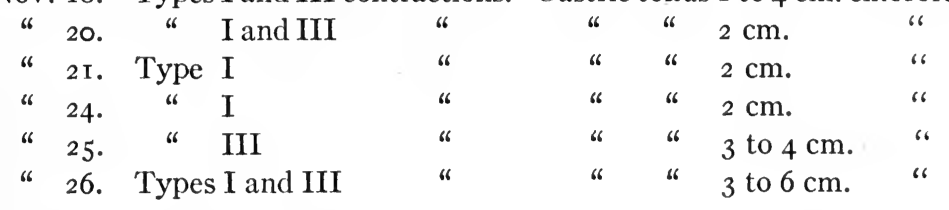

The reader will note that in both dogs the hemorrhage induced temporarily a greater gastric tomus and intensity of hunger contractions 
than those typical for these dogs before the hemorrhage. This effect of the hemorrhage disappears in less than 24 hours. The controls show that the stimulation of the gastric tonus mechanism is due to the hemorrhage, and is not an after-effect of the ether anesthesia. That they were felt as hunger contractions by the dogs was evidenced by the amount of food consumed on the hemorrhage days.

The following considerations might be offered, not only as a possible, but also as a probable explanation. The blood is, of course, the purveyor of nutritive substances to all the tissues of the body. Its chemical composition is kept remarkably constant. If

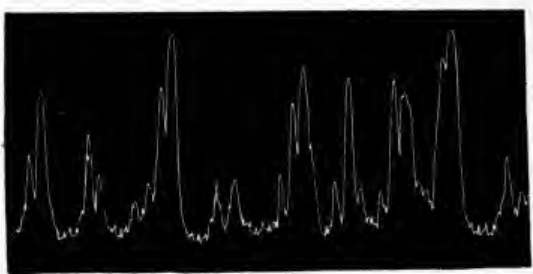

A

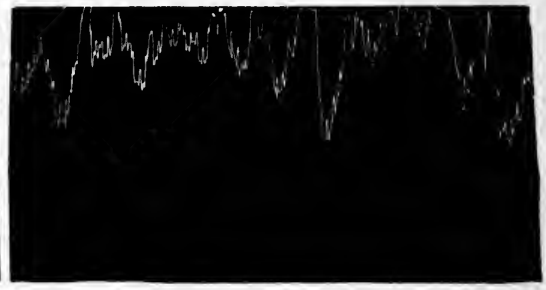

B

FIG. 28.- $A$, tracing showing gastric tonus and type I hunger contractions characteristic of dog before hemorrhage. $B$, record of gastric tonus contractions of $\operatorname{dog} 60$ minutes after drawing 169 c.c. blood from the carotid artery; showing the temporary stimulation of the gastric hunger mechanism as an after-effect of excessive hemorrhage (bottom of tracing $=\circ \mathrm{mm}$. pressure) (Luckhardt).

now an animal is bled extensively ( 2 to 3 per cent of body weight), there is removed suddenly an enormous amount of pabulum, that is, of those various substances which are taken up by the different tissues during circulation. The organs and tissues deprived of these respective nutritive substances become hungry (call for food) by giving up a something (a hormone) which acts on the neuromuscular apparatus of the stomach to produce the hunger contractions.

We recognize, of course, that acute hemorrhage introduces other factors. Some of them have been mentioned. The explanation offered gives a simple, reasonable picture of the mechanism involved. By acute hemorrhage we induce sudden acute starvation. Probably all the tissues of the body give up this "hunger hormone." By withholding food from the animal the blood changes appear 
more slowly, depending for one thing on the state of nutrition and reserve food supply of the animal before the period of starvation is started.

\section{HUNGER CONTRACTIONS OF THE STOMACH POUCH ISOLATED ACCORDING TO THE METHOD OF PAVLOV}

It was hoped that simultaneous record of the contractions of the stomach pouch and of the main stomach would throw some light on the relative importance of the tonus of the vagi, the condition of the blood, and the physiological state of the gastric motor mechanisms in the genesis of the hunger contractions. The Pavlov operation leaves the vagi connections with the stomach pouch at least partially intact, so that if the hunger contractions are normally initiated by efferent vagi impulses we might expect a close parallel between the rate and intensity of the contractions in the two stomachs. The character of the blood flowing to the two stomachs is necessarily the same. The amount of local nervous co-ordination between the two stomachs depends on the extent of the intact myenteric plexus and muscularis. The operation severs by far the greater amount of these neuromuscular connections. This may diminish the local nervous co-ordination and thus permit the development of different physiological states of the motor mechanisms in the two stomachs. The work was done on two young and vigorous dogs. Relatively large stomach pouches were made according to the method of Pavlov. In Dog I the muscularis joining the two stomachs was left intact for a distance of $6 \mathrm{~cm}$. These figures were verified by post-mortem examination at the end of the experiment.

Simultaneous records of the hunger contractions in the two stomachs were taken while the dogs were lying quietly and comfortably in the lap of an attendant. The balloon was passed into the main stomach via the esophagus. The balloon used for the stomach pouch was much smaller than that used for the main stomach.

Results.-Dog I, having the $6 \mathrm{~cm}$. of intact muscularis and myenteric nerve plexus uniting the two stomachs, showed a fairly 
close parallel of the tonus and the hunger contractions of the main stomach and the stomach pouch. When the tonus of the stomach was so great that the type III contractions (or incomplete tetanus) were present, the synchrony appeared complete. The two stomachs gave contractions of the same strength and rapidity. The contraction and relaxation phases of the individual contractions show also a fair degree of correspondence.

When the gastric tonus is weaker, so that the stomach exhibits the slower and stronger contractions of type II, the parallel between the stomachs is still in evidence, but it is less complete. That is to say, the contractions may appear simultaneously and be of corresponding strength, they may appear simultaneously and be very unequal in strength, or there may be considerable lack of synchrony both in the beginning and in the duration of the contractions of the two stomachs. At times the pouch would give two separate and strong contractions during a single but more protracted contraction of the main stomach. When the contractions are still slower, or of type I, the co-ordination between the two stomachs is more nearly perfect.

Dog II, with only $3 \mathrm{~cm}$. of intact muscularis and myenteric plexus uniting the two stomachs, exhibited no synchrony between the two stomachs at any time. The main stomach would be quiescent, while the pouch showed vigorous hunger contractions, or vice versa. But more frequently both stomachs exhibited hunger rhythm at the same time, but without any synchrony in the rate and the strength of the contractions.

The Pavlov operation necessarily severs a considerable portion of the vagi connection with the pouch. But it is well known that at least half of the vagus influence can be eliminated by section of one vagus without any appreciable disturbance of the gastric tonus. In general the hunger rhythm of the pouch in Dog II resembled that of the stomach of dogs with section of both vagi. In this animal (Dog II) the amount of vagi connections with the motor mechanism of the pouch was not sufficient to maintain the normal tonus. It is also evident that the $3 \mathrm{~cm}$. bridge of myenteric plexus was also insufficient for local co-ordination of the two stomachs. 
In Dog II, therefore, the two stomachs differed in the quantity of innervation. The other obvious differences between the main stomach and the pouch, such as the presence of saliva, the occasional presence of intestinal juice and bile, gases and food débris, hair, etc., were common for Dogs I and II. These conditions were not sufficient to create inco-ordination through differences in the physiological state of the motor mechanisms when the connecting bridge of myenteric plexus was $6 \mathrm{~cm}$. in length. The fact that in Dog II the pouch would show the hunger contractions during complete quiescence of the main stomach and vice versa seems to show that the physiological state of the gastric motor mechanism and not the character of the blood is the primary factor in the genesis of these contractions. The main stomach and the pouch were supplied with the same blood. The character of the coordination of the two stomachs in Dog I indicates that the hunger contractions are not normally caused by periodic impulses from the brain via the vagi. If such were the case there should have been a closer synchrony of the contraction and relaxation phases in the main stomach and the pouch. A primary vagi innervation of the contractions would not permit a contraction in the pouch with no contraction in the main stomach, the beginning of the pouch contraction during the relaxation phase of the main stomach, or two distinct and strong contractions of the pouch during a single contraction of the main stomach. These results are readily explainable on the basis of local genesis of the contractions and some impairment of the myenteric connections between the two parts. Under these conditions the physiological state of the motor mechanism of the two stomachs would not be exactly alike, and in consequence there will be some interference with, or inhibition of, the excitation wave at the isthmus joining the two parts, as well as in the two stomachs themselves. Thus the excitation wave from the main stomach may pass the myenteric bridge unimpeded, but may reach the pouch during the refractory phase of the latter, and thus produce little or no effect. And a similar interference may obtain in the case of the excitation waves from the stomach pouch. Since most of the myenteric plexus joining the two parts of the stomach 
is severed in the Pavlov operation, the local co-ordinating mechanism is obviously imparied, although not completely destroyed. And if we assume a peripheral origin of the hunger contractions, this must lead to a certain degree of independent activity of stomach and pouch. The results demanded by this assumption of a peripheral stimulus or local automatism initiating the hunger contractions are those actually found in Dog I, that is, impaired synchrony of the two parts. As we have seen, the synchrony of the two stomachs is more nearly perfect the slower the contractions. When the contractions come at longer intervals there is less chance for interference with the excitation wave in the region of the myenteric isthmus and of collision, so to speak, with the refractory state. The parallel in the activity of the two stomachs during type III contractions may be only apparent or a parallel of the tonus only, for when the tonus reaches a certain degree the contractions appear at their maximum rapidity. Hence if the main stomach and the pouch have equally strong tonus they will exhibit an equal number of contractions per unit of time, even without any physiological co-ordination of the excitation waves between the two stomachs.

\section{SUMMARY}

Blood from starving animals and animals in pancreatic diabetes transfused into normal animals acts as a temporary stimulus to the gastric hunger mechanism.

Excessive hemorrhage is followed by a temporary augmentation of the gastric hunger contractions.

Prolonged starvation, pancreatic diabetes, and possibly excessive hemorrhage result in some change in the blood that acts as a stimulus to the gastric hunger mechanism.

The character of the parallel between the hunger contractions of the main stomach and of the stomach pouch supports the view that these contractions are caused primarily by a gastric automatism and not by motor impulses via vagi nerves.

When the muscularis and myenteric isthmus joining the main and the accessory stomachs is relatively narrow, the two stomachs 
exhibit complete independence of the hunger contractions, even to the point of vigorous activity of the one during quiescence of the other. This fact points to a local automatism as a primary factor rather than the condition of the blood, as the character of the blood flowing to the main stomach and the stomach pouch is necessarily the same.

\section{ACTION OF EPINEPHRIN, PITUITRIN, ORGAN EXTRACTS,} AND DRUGS

The fact that the gastric hunger contractions involve essentially the same motor mechanisms as the gastric digestion contractions suggests that the chemical control exerted by the blood is probably the same on both. Esserine and pilocarpin augment the hunger contractions, while morphine produces profound inhibition (Luckhardt). Pituitrin produces an initial augmentation; epinephrin, amyl nitrite, calcium chloride, etc., a temporary depression.

Substances that cause temporary augmentation of the gasterointestinal movements can apparently be prepared from all the tissues of the body. Attempts have been made to show that there is a specific "motor hormone" for gastero-intestinal peristalsis in the wall of the digestive tract itself. With the exception of epinephrin it is probable that all the substances so far studied in the various tissue extracts are abnormal split products or artifacts not present in normal blood, and hence playing no rôle either in the normal hunger contractions or in the digestion peristalsis. Biedl was not able to demonstrate any favorable action of spermin or testicle extracts on metabolism, appetite, or hunger. The fact that a drug or a tissue extract when introduced hypodermically or intravenously initiates or augments the gastric hunger contractions does not imply that these drugs or extracts have the same effect on the sensation of hunger. The latter effect may be modified or abolished by other actions, peripheral and central, of these substances.

The work of Bayliss and Starling, Magnus, Cannon, and others seemed to show that the gastero-intestinal contractions are primarily local reflexes through the Auerbach's plexus or initiated by 
"automatic" nervous discharges from the ganglionic plexus. Kieth reports the presence in the Auerbach's plexus of "nodal" tissue similar to that in the heart, and he ascribes the main rôle in the genesis of the gastero-intestinal movements to this nodal tissue. The presence of nodal tissue in the Auerbach's plexus does not materially alter the problem of the causation of the gasterointestinal movements.

The special difficulties in relating the hunger contractions of the stomach to chemical changes in the blood are the periodicity of the rhythm and the abrupt cessation of the contraction periods. So far as we know today chemical changes or starvation changes in the blood are more continuous. Of course, we do not deny the possibility of a periodic secretion into the blood of some specific substance or hormone having this effect on the stomach, but all the evidence so far at hand is against this possibility. The fact that after extirpation of the parathyroid gland there is a tendency to atonicity and motor paralysis of the gastero-intestinal tract, especially when the symptoms of tetany are severe, is no evidence that the parathyroid secretion is a motor stimulus to the stomach and intestines. The motor paralysis is probably due to secondary causes. The only conclusion that seems warranted by the facts at hand is that the gastric hunger mechanism is primarily automatic or independent of blood changes as well as of central nervous influences; but in the normal individual chemical changes of the blood as well as nervous impulses from the brain and spinal cord augment or decrease this primary automatism in a way to correlate it with the needs of the organism.

This conclusion should not be regarded as a bar to further investigation of the chemical control of the hunger mechanism in health, and especially in disease. Such work is not only urgent, but is certain to yield important results. Recent experiments by Dr. Luckhardt seem to show that the gastric hunger contractions in dogs are augmented by inducing the condition of phlorhizin glycosuria. It is significant that all the conditions which have so far proved to increase the hunger contractions (diabetes mellitus, 
pancreatic diabetes, prolonged starvation, great physical exertion, extreme cold, phlorhizin glycosuria) have these two things in common: (I) acidosis of varying degrees, and (2) either inability to use sugar by the tissues or else a lessened amount of sugar available for the use of the tissues because of the sugar having been oxidized or eliminated. 


\section{CHAPTER XIV}

\section{SECRETION OF APPETITE GASTRIC JUICE IN MAN}

\section{THE FLUID CONTENTS OF THE STOMACH FREE FROM FOOD}

The normal stomach, empty of food, always contains some fluid and mucus. The stomach is therefore, strictly speaking, never empty. This fluid in the empty stomach is made up of (I) gastric juice and mucus, (2) saliva, (3) duodenal contents (pancreatic juice and bile). Pancreatic juice and bile are frequently absent, however. The total fluid content of the empty stomach as well as the chemistry of this fluid depend on several factors, such as the relative rate of gastric and salivary secretion, the tonus and contractions of the stomach, the rate of absorption in the stomach, and the rate of emptying of the stomach contents into the duodenum.

According to the more recent literature the fluid content of the empty stomach of normal persons varies within wide limits. Verhagen found the average to be ro to 25 c.c., but occasionally as much as 50 c.c. were obtained. Moritz gives higher figures, or 24 to 64 c.c. Working on himself, Moritz obtained an average of 43 c.c. of fluid in the stomach in the morning, with an acidity of o.I I per cent. Rehfus, Bergheim, and Hawk state that in normal persons the fluid in the empty stomach in the morning varies from 30 c.c. to I80 c.c. The average of more than two hundred observations on our gastric-fistula case, Mr. V., is 20 to 25 c.c. In Mr. V. the salivary factor is excluded as the esophagus is completely closed. The fluid content in the stomach in the morning before breakfast is greater than at noon before lunch. This is probably due to a lower tonus of the stomach in the morning. Sixty tests on eight normal medical students in the author's laboratory showed a variation from to to I 20 c.c., with an average of 40 c.c. Some individuals tend to run high; others are consistently low. 
II. CONTINUOUS SECRETION OF GASTRIC JUICE IN THE EMPTY STOMACH OF NORMAL' PERSONS

Continued secretion of gastric juice in the absence of food in the alimentary tract, and in the absence of cerebral processes relating to appetite ("psychic" stimulation), is a well-known phenomenon in certain types of gastric disorders, but it is generally assumed by physiologists that, in the absence of psychic stimulation, the gastric glands cease to secrete almost as soon as the stomach is emptied of chyme, and that the glands remain quiescent up to the next feeding. The quiescence is supposed to be sufficiently complete to render the surface of the stomach alkaline, due to the continued secretion of alkaline mucus. To the extent that this view is anything more than an assumption, it is based essentially on the studies by Pavlov and his pupils on dogs. Pavlov frequently emphasizes the fact that not a drop of gastric juice flows from the stomach unless there is food or other stimuli in the stomach or unless the appetite is called into play. Later Boldyreff reported that on continued starvation the gastric glands exhibit periodic activity, and if the starvation is maintained for more than three or four days the secretion of the gastric gland becomes continuous. In gastric-fistula cases of normal persons no specific study has been made of the continuous secretory activity of the empty stomach, but in some instances (Kaznelson, Hornborg) there are indications of a slow, continued secretion even when the stomach had been free from food for hours.

Most of our observations on Mr. V. were made between Io:०० A.M. and 4:00 P.M., the usual breakfast of coffee, milk, and biscuits being taken at 7:00 A.M. A few tests were made between 9:00 A.M. and I2:00 M., and on such occasions Mr. V. did not take any breakfast. The rate of the continuous secretion of gastric juice in the empty stomach of $\mathrm{Mr}$. V. varies from a few cubic centimeters up to 60 c.c. per hour.

In general more gastric juice is obtained from the empty stomach if the stomach is emptied (through the fistula) every 5 or to minutes than if it is emptied every 30 or 60 minutes. It is therefore likely that some of this secretion passes into the intestines or is 
actually reabsorbed in the stomach itself. It does not seem probable that the presence of a certain amount of this juice in the stomach would tend to inhibit further secretion.

If the secretion rate is low the acidity is usually not over 0.20 to 0.25 per cent, but the pepsin concentration is nearly as great as that of the appetite gastric juice. If the secretion rate is moderate the acidity is greater and the pepsin concentration may even exceed that of the appetite secretion. When the secretion rate is low the juice is very thick and opalescent, owing to the great amount of ropy mucin.

What constitutes the stimulus to the continuous gastric secretion? We think it can be shown that it is not an appetite secretion. To be sure, in the case of normal and vigorous persons, periods of hunger and appetite are present almost as soon as the stomach is emptied of food. And it is obviously difficult so to control the cerebral processes of a person that the thoughts are not diverted to food and eating, especially if the usual meal time has passed and one's attention is at the time on the stomach. This is especially true if the gastric juice is collected every ro minutes. If the stomach is emptied every 30 or 60 minutes and the person is kept very busy with matters not pertaining to food and eating, we think this factor is entirely eliminated. This was done every day for two weeks at a stretch, so as to make it a mere incident or routine in the day's work. Nevertheless, the continued secretion persisted with the usual fluctuations in character and quantity.

Is the secretion due to a subconscious secretory vagus tonus? The vagi carry secretory fibers to the gastric glands. But we know next to nothing about the reflex or tonus control of this neurosecretory mechanism. We know that the vagi send tonus impulses to the gastric motor mechanism. But it does not follow that this is also the situation in regard to the gastric gland.

The presence of food in the intestine may be partly responsible for this continued secretion, by reflex action from the intestinal mucosa (Pavlov), or by absorptions of gastric secretins into the blood. In a thirty-nine-year-old man with gastric fistula Umber obtained some secretion of gastric juice on rectal feeding with milk, 
sugar, and eggs. Umber explains the secretion as a reflex effect from the mucosa of the large intestine. We are not convinced that purely psychic factors are excluded in his experiments. If a person is hungry it is likely he will be led to think of food and eating by the mere act of rectal feeding. Moreover, Umber's experiments were not numerous enough really to establish the point.

Gastric juice itself contains mucins and proteins that are digested by the pepsin-hydrochloric of the gastric juice. It is highly probable that the products of this digestion yield gastric secretagogues, just as in the case of some of the digestion products of the food proteins. According to Bickel amino-acids given by mouth cause secretion of gastric juice. Absorbed slowly in the stomach or passed into the intestines to be absorbed there, the products of the auto-digestion of the gastric juice probably furnish chemical stimuli for a slow but continuous gastric secretion. Which one of these factors is of prime importance in the continuous secretion of gastric juice by the empty stomach must be determined by other lines of work, especially in disease conditions where the continuous secretion is greatly increased.

\section{APPETITE SECRETION OF GASTRIC JUICE}

I. The mere act of chewing indifferent substances and the stimulation of nerve-endings in the mouth by substances other than those directly related to food cause no secretion of gastric juice. On this point our results on Mr. V. are in complete accord with those obtained from dogs by Pavlov and his school, and contrary to those of a number of observers on man.

In a woman with gastric fistula and esophageal stenosis Richet reports secretion of gastric juice from acid stimulation in the mouth. $\mathrm{He}$ also states that the introduction of food or sapid substances into the stomach via the fistula caused salivation. This must have been a purely psychic effect, unless the procedure caused nausea. The subject was evidently a hypersensitive woman. We have never observed any of these effects in Mr. V.

In 1896 Schüle introduced the method of obtaining pure appetite gastric juice in man by emptying the stomach by means of a stomach 


\section{CONTROL OF HUNGER IN HEALTH AND DISEASE}

tube, then chewing food for 15 minutes, and again emptying the stomach with the tube. He claims that the mere act of chewing and the tasting of such sapid substances as oil of peppermint, slices of lemon, and mustard cause secretion of gastric juice even in the absence of appetite. Troller, using Schüle's method, also reports that slices of lemon, mustard, etc., in the mouth, as well as the mere act of chewing, cause secretion of gastric juice. In the majority of his experiments the secretion thus obtained was very slight (only about one-fourth that obtained on chewing bread), and in some of

TABLE I

Gastric Juice in c.c. (Mr. V.)

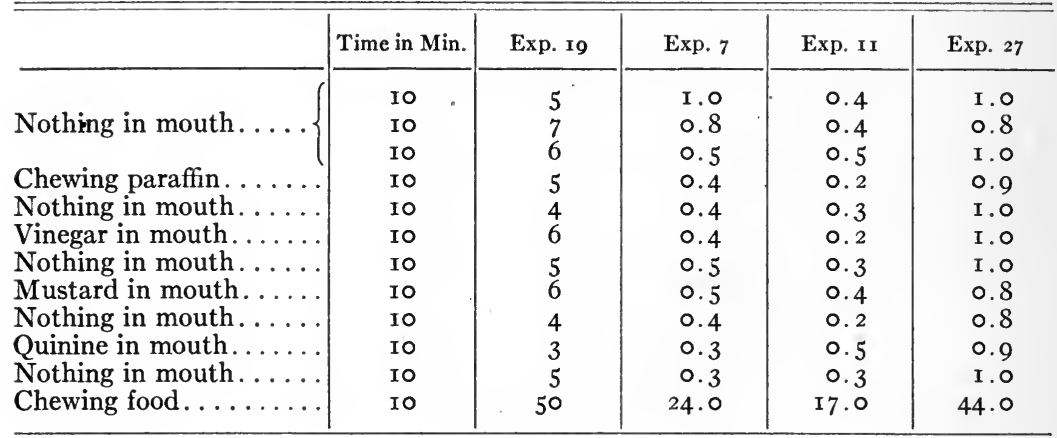

the experiments recorded in detail the acidity of the juice is so low that it must have been mixed with swallowed saliva. It is probably very difficult for the average person to avoid swallowing some saliva with mustard or citric acid in the mouth for ro to I 5 minutes. Troller did not adequately control the rate of the continuous secretion in the empty stomach when the persons had nothing in particular in the mouth. Riegel cites the case of a professional cook, in whom chewing of food (beefsteak) or slices of lemon caused no secretion of gastric juice. This man showed chronic digestive disorders, however. But Riegel suggests that the absence of appetite secretion was due to a kind of permanent fatigue of the tastesecretory mechanism in consequence of his duties as cook. Hornborg, working on a five-year-old boy with gastric fistula and nearly 
complete cicatricial stenosis of the esophagus, concluded that chewing indifferent, badly tasting, or strongly tasting (lemon) substances did not induce secretion of gastric juice. Umber obtained no gastric secretion by chewing indifferent substances (pieces of rubber), but in one experiment alcohol in the mouth gave a slight secretion. It must be noted that Umber's subject was a man fifty-nine years old, who might have been in the habit of taking alcoholic beverages with his meals.

Kaznelson and Bickel, working on a twenty-three-year-old girl with gastric fistula and complete cicatricial esophageal stenosis, report that all sapid substances (quinine, asafoetida, etc.) in the mouth, even those that give rise to disgust, initiate or augment the gastric secretion. Kaznelson cites one experiment with quinine (control experiment with water), from which she concludes that bitter substances in the mouth augment the secretion of gastric juice, but her actual figures show, if anything, the reverse. The total secretion of gastric juice for 80 minutes with the water control (sham drinking) was 43.7 c.c., while the quinine experiment yielded only 37.6 c.c. for the corresponding time.

How are these contradictory findings to be accounted for? In view of the consistently negative results of Pavlov and his students on dogs, and of Hornborg, and the writer on man, it is our belief that the investigators who report that mechanical chewing and general stimulation of the nerve-endings in the mouth cause secretion of gastric juice have not eliminated the factors of appetite, swallowed saliva, and variations in the rate of the continuous secretion of the empty stomach. In man the appetite factor is not easily controlled, except by a long series of tests in which the experimental procedure becomes a mere routine to the subject. There appears to be no direct or unconditional reflex pathway from the mouth to the gastric gland. Unless the stimuli in the mouth initiate or augment the central processes that constitute the sensation of appetite, there is no innervation of the secretory nerve-fibers to the gastric gland.

2. The relatively slight and inconstant secretion of gastric juice produced by seeing, smelling, or thinking of food.-Bringing a tray 
of palatable food into the room in sight of Mr. V. has never yet caused secretion of gastric juice, no matter what the degree of hunger and appetite. It is probable that under these conditions the primary and normal effects of seeing and smelling the food are inhibited by the consciousness of the experiment, or possibly his main interest was not the food, but the expiration of the experiment so that he might partake of the food. In order more closely to approximate normal conditions, Mr. V. was sent out to the near-by cafeteria to select the lunch that he knew he would eat shortly after returning with it to the laboratory. The rate of his gastric secretion was measured for ro-minute periods before going for the food, during the selection of, and after returning to the laboratory with it. In the majority of these tests the act of selecting the ingredients for the noonday meal caused a slight and temporary augmentation of the secretion rate of the empty stomach. On the whole this augmentation was greater the greater the rate of the continuous secretion. But on some days the augmentation was absent, although Mr. V. was in good health, felt hunger, and the cafeteria displayed the usual variety of food.

Pavlov reports that there are great individual variations in dogs in the amount of gastric secretion induced by seeing and smelling food. This, in all likelihood, is true of man, and we suspect that Mr. V. belongs to the group of individuals in whom the taste of the food is the all-important factor in the psychic secretion of gastric juice. We have not been able appreciably to augment the continuous secretion in Mr. V. by inducing the thought of food, for example, during a test while he is busy with other work, by arresting his attention casually, and by discussing with him the taste and ingredients of his favorite dishes.

Schüle states that seeing or smelling food causes no secretion of gastric juice in normal persons. This is directly contradicted by Bulawinzew. This investigator emptied the stomach by means of the stomach tube, then let the subject see or smell food, and again emptied the stomach. The gastric juice thus obtained had such low acidity $(0.2$ per cent $\mathrm{HCl})$ that it must either have been in the 
continuous gastric secretion or the appetite gastric juice mixed with saliva. There is nothing in the review to indicate that he controlled the continuous gastric secretion. Hornborg obtained no secretion of gastric juice from a five-year-old boy on his seeing or smelling food, probably because the child always became angry when not permitted to eat the food shown him. Cade and Latarjet report secretion of gastric juice was induced by talking to the subject about her favorite food. This subject (a young woman) is exceptional in that she virtually had an accessory stomach, but

TABLE II

Secretion of Gastric Juice (Mr. V.) on Seeing, Smelling, and Thinking of Food when Hungry

\begin{tabular}{|c|c|c|c|c|c|c|c|}
\hline & \multirow{2}{*}{$\begin{array}{l}\text { TIME } \\
\text { IN } \\
\text { MIN. }\end{array}$} & \multicolumn{6}{|c|}{ GaStric JUICE IN C.c. } \\
\hline & & Exp. 3 & Exp. 8 & Exp. I 2 & Exp. 15 & Exp. 30 & Exp. 45 \\
\hline & I0 & 5 & 0.5 & 0.3 & 0.4 & 0.6 & 0.4 \\
\hline & IO & 7 & 0.3 & 0.4 & 0.5 & 0.5 & 0.3 \\
\hline & Io & 6 & 0.5 & 0.3 & 0.4 & 0.4 & 0.4 \\
\hline \multirow{4}{*}{$\begin{array}{l}\text { Selecting the lunch at the cafe- } \\
\text { teria } \ldots \ldots \ldots \ldots \ldots \ldots \ldots\end{array}$} & IO & I4 & I.O & I.O & $3 \cdot 5$ & 0.5 & 1.0 \\
\hline & IO & IO & I.O & 0.6 & 2.0 & 0.4 & I.O \\
\hline & 10 & 5 & 0.5 & 0.5 & $\ldots$ & 0.3 & I.O \\
\hline & Io & 6 & 0.3 & 0.4 & $\ldots$ & 0.4 & 0.7 \\
\hline
\end{tabular}

the mucosa of the isolated stomach portion was directly exposed so that the collection of the secretions was rather difficult. Kaznelson and Bickel, working with a twenty-three-year-old girl with gastric fistula and stenosis of the esophagus, reached the remarkable conclusion that anything which stimulated the olfactory sense induced secretion of gastric juice in the resting stomach. Thus they claim that smelling ammonia, acidic acid, and aromatic oils causes secretion of gastric juice. This we are absolutely unable to confirm on Mr. V. It is possible that in this young woman every gustatory and olfactory stimulus when manipulated by the investigators led to thoughts of food through idea associations. 


\section{GASTRIC SECRETION INDUCED BY TASTING AND CHEWING PALATABLE FOOD}

I. The secretion rate.-We have records of ${ }_{15} 6$ tests of the appetite secretion of Mr. V. during the 20 minutes' mastication of the noonday meal. The particular ingredients of this meal were of his own selection, and varied from day to day. The meal usually included soup and some kind of meat and gravy, and always milk and a dessert. Secretion of gastric juice during 20 minutes' mastication of palatable food was: lowest, 30 c.c.; highest, I 56 c.c.; average, 70 c.c.; number of experiments, 156 .

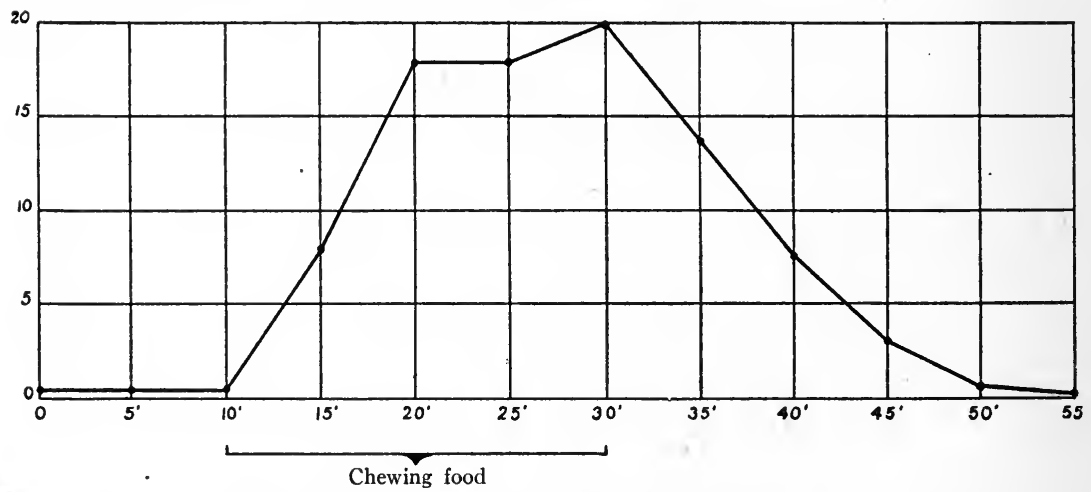

FIG. 29.-Typical curve of secretion of gastric juice of Mr. V. on mastication of palatable food for 20 minutes. The gastric juice was collected at 5 -minute intervals. The rise in the secretion rate during the last 5 minutes of mastication is due to chewing the dessert (fruit).

This gives an average rate of secretion of 3.5 c.c. of gastric juice per minute. The maximum rate of secretion determined at any time was 54 c.c. in 5 minutes, or Io. 8 c.c. per minute; the lowest was 7 c.c. in 5 minutes, or $\mathrm{r} .4$ c.c. per minute. The secretion rate is proportional to the palatability of the food. Thus the secretion rate is nearly always highest in the last 5 -minute period, when Mr. V. masticates the dessert, and on the day when the highest rate of secretion was noted ( 156 c.c. in 20 minutes) Mr. V. stated that the lunch was "unusually fine."

Is this rate, and quantity of appetite secretion of gastric juice typical for normal adults? Mr. V. is in normal health, except for 
infrequent periods of headache and nervousness, the etiology of which is obscure. 'Troller reports 5 experiments on a person with nervous dyspepsia. Chewing beefsteak for I $_{5}$ minutes yielded 55 c.c. of gastric juice, while 3 experiments on a person with hyperacidity gave 50 c.c. gastric juice in 15 minutes. This is a secretion of a rate of about 3.5 c.c. per minute. Chewing bread for I 5 minutes yielded much less gastric juice. In the case of persons with hypochlorhydria the average secretion for I 5 minutes (chewing beefsteak) was only 28 c.c. In Umber's fistula case (a man fifty-nine years old) two tests with chewing beefsteak for 15 minutes yielded

TABLE III

Rate of Appetite Secretion of Gastric Juice of Mr. V.

Detail of Typical Experiments

\begin{tabular}{|c|c|c|c|c|c|c|c|c|c|c|}
\hline \multirow{3}{*}{$\begin{array}{l}\begin{array}{c}\text { EXPERIMENT } \\
\text { No. }\end{array} \\
20 \ldots \ldots\end{array}$} & \multicolumn{10}{|c|}{ Rate of Secretion of Gastric Juice in Consecutive 5-Min. Periods. C.c. } \\
\hline & \multicolumn{3}{|c|}{$\begin{array}{l}\text { Before Starting } \\
\text { Chewing }\end{array}$} & \multicolumn{4}{|c|}{ During Chewing } & \multicolumn{3}{|c|}{$\begin{array}{l}\text { On Cessation of } \\
\text { Chewing }\end{array}$} \\
\hline & I & 0.5 & 0.8 & IO & I 5 & I4 & 20 & 5 & 3 & 1.0 \\
\hline $31 \ldots \ldots$ & $\ldots$ & $\ldots$ & $\ldots$ & I I & 18 & 17 & 23 & IO & 6 & 2.0 \\
\hline $35 \ldots \ldots$ & 0.5 & 0.6 & 0.7 & I 5 & I6 & 15 & I8 & 8 & 4 & I. 5 \\
\hline $55 \ldots \ldots$ & 0.3 & 2.0 & 3.0 & 20 & 22 & $2 I$ & 30 & I5 & 6 & 6.0 \\
\hline $86 \ldots . .$. & 0.2 & 0.2 & 0.3 & 5 & 20 & I8 & 20 & 9 & 3 & 1.0 \\
\hline $94 \ldots \ldots$ & 0.2 & 0.3 & 0.2 & 6 & I I & I 5 & I 2 & 3 & 2 & 0.5 \\
\hline $120 \ldots \ldots$ & 0.2 & 0.2 & $0 . I$ & 6 & 28 & 20 & 29 & 8 & 6 & 2.0 \\
\hline I $50 \ldots .$. & 0.2 & 0.3 & 0.2 & 22 & 54 & 35 & 45 & 20 & I5 & 8.0 \\
\hline
\end{tabular}

73 c.c. and 48.5 c.c. gastric juice in 60 minutes. This low rate of secretion (about I c.c. per minute) must be due to the advanced age and to a malignant tumor of the esophagus. The ten-year-old girl studied by Sommerfeld secreted I Io to I 50 c.c. gastric juice in 90 minutes on chewing meat or mixed food for 30 to 40 minutes, a secretion rate of 2 to 2.5 c.c. per minute. The maximum secretion rate in the twenty-three-year-old girl studied by Kaznelson and Bickel was 5 c.c. per minute, the average secretion rate being much lower. Hornborg's five-year-old boy secreted $I_{5}$ to 25 c.c. in 30 minutes on chewing meat or apple pie. Chewing bread or milk yielded less than half this amount. The three-year-old child observed by Bogen, on chewing meat for I 5 minutes, yielded 6 to 
22.5 c.c. gastric juice, or an average rate of less than I c.c. per minute.

These data reported by previous investigators cannot be directly compared with our results on Mr. V. for the reason that the collection of the gastric juice was not always confined to the actual period of mastication of the food. The rate of the appetite secretion starts to fall almost as soon as Mr. V. ceases to masticate the food, and in ${ }_{15}$ minutes the activity of the gastric glands is in most cases down to the level of the continuous secretion. The secretion rate is highest during the actual tasting of the food.

In this respect there is a marked difference between man and dog. In the dog, after I 2 to 24 hours of starvation, sham feeding with meat for 5 minutes may initiate and keep up secretion of gastric juice for 3 to 6 hours (Pavlov, Rosemann). It is obvious that in these tests on dogs the starvation period was much longer and the hunger and appetite more intense than in our experiments on Mr. V. Another factor is probably the greater voluntary control over attention and other cerebral processes in man.

It may be of interest in this connection to note the rates of gastric secretion that have been obtained by sham feeding in dogs. Konowaloff reports 4 c.c. per minute; Schoumow-Simanowsky found a maximum of 5 c.c. per minute; and Rosemann (in a dog weighing $24 \mathrm{~kg}$.) gives as the average 3.4 c.c. per minute. Since the quantity of gastric glands even in very large dogs is probably only a third of that in the adult man, the foregoing data seem to indicate that the gastric glands in dogs work with greater speed than the gastric glands of man.

2. The direct relation between the rate of appetite gastric secretion and the palatableness of the food.-The mastication of bread and butter or the taking of milk in the mouth yields much less gastric juice than the chewing of meat. This is in line with results of previous observers on man. The taste nerve-endings are evidently stimulated more intensely by the readily diffusible sapid substances in the meat. In general the desserts (pies, pudding, fruits) yielded even greater secretion than meat. This was particularly noticeable in the case of chewing oranges. Mr. V. is especially fond of oranges. 
The sapid substances in the orange juice probably diffuse readily and thus reach all the taste nerve-endings in marked concentration. There is no question but that the mastication of a palatable dessert at the end of a meal serves to augment and prolong the appetite secretion of gastric juice.

3. The latent period of the gastric appetite secretion.-Pavlov and his coworkers found that the appetite gastric secretion in dogs exhibited uniformly a latent period of 5 to 6 minutes. According to the literature the latent period of the appetite gastric-juice secretion in man varies from 3 to 9 minutes. The latent period depends primarily on the condition of the gastric glands. Thus if there is a continuous gastric secretion of 2 to 6 c.c. per ro minutes at the time mastication of the food begins, the appetite secretion shows practically no latent period at all. The quantity of gastric juice secreted during the first 5 minutes of chewing is just as great as that secreted during the second or third 5-minute periods. On the other hand, if the continuous secretion is very low ( 0.2 to 0.3 c.c. per Io minutes) the appetite secretion shows a latent period of 2 to 4 minutes. It is therefore evident that with the gastric secretion already in progress the appetite secretion reflex exhibits no greater latent time than neuromuscular reflexes in general.

The latent period varies indirectly with the intensity of the appetite stimulation. If the continuous secretion is very low, the latent period of the secretion does not exceed 2 to 3 minutes, provided the food is very palatable.

\section{TOTAL SECRETION OF GASTRIC JUICE IN MAN ON AN AVERAGE MEAL}

As stated above, Mr. V. yields appetite gastric juice at: minimum secretion rate, 84 c.c. per hour; maximum secretion rate, 648 c.c. per hour; average secretion rate, 2 10 c.c. per hour. Does this furnish us a clew to the total gastric secretion on an average meal in man? This question cannot be answered by direct measurements; even in cases of duodenal fistula and collection of all the chyme issuing through the pyloric opening, as the alimentary tract of such persons is far from normal, and we still have the variable factors of swallowed saliva and of direct absorption in the stomach. 
In the case of dogs sham feeding alone may yield 600 to 700 c.c. of gastric juice in 4 to 6 hours. But this situation is abnormal because the sham feeding does not satisfy the appetite, even though the secretion inhibits the hunger. It is therefore certain that the appetite secretion is much less when the food is. permitted to reach the stomach. But when the food is allowed to reach the stomach, how can we measure the total gastric secretion? Using large dogs with fistula of duodenum, Moritz reports that the ingestion of $200 \mathrm{gm}$. of meat caused a secretion of 320 c.c. gastric juice in 7 hours. Part of this was undoubtedly swallowed saliva, and possibly some admixture of bile and pancreatic juice. With the same method Tobler obtained 200 to 300 c.c. of gastric juice from feeding $100 \mathrm{gm}$. meat; part of this fluid was undoubtedly swallowed saliva.

It seems to us that we can arrive at a very close estimate of the total average secretion of gastric juice in a man on the following basis: Pavlov and his pupils have shown on dogs that the secretion curves of the main and the accessory stomach pouch run parallel. They have also shown that on a meal of meat, or a mixed meal, the secretion usually reaches the maximum toward the end of the first or during the second hour. Lönnquist notes particularly that the secretion does not reach its maximum until toward the end of the second hour after eating. On the whole, the quantity of gastric juice yielded by a dog's accessory stomach after the first two hours following a moderate meal of meat, bread, or a mixture of meat and bread, is about half of that secreted during the entire digestion period. This is evident from experiments reported in detail by Pavlov and his students, as well as from studies on dogs in our laboratory. But this is not true if a very large quantity of food is given, or if the food contains a considerable amount of fat, as in both cases the secretion of fluid is greatly prolonged.

We can safely assume that the general relations and the relative importance of the appetite and the hormone gastric juice are the same in man and dog. Pflaunder supports the view that the maximum rate of secretion in man is reached at the end of the first or the beginning of the second hour of digestion. Sick finds that the maximum acidity of gastric content is usually reached at the 
end of the first hour of digestion. The same is shown by the more recent studies of Rehfus, Bergheim, and Hawk, using the Ewald rest meal on normal persons.

The total secretion of gastric juice in normal adult man on ingestion of the average dinner of meat, bread, vegetables, coffee or milk, and dessert will, on the foregoing assumptions, be as follows: ist hour, 200 c.c. gastric juice; $2 \mathrm{~d}$ hour, 150 c.c.; $3 \mathrm{~d}$ to 5 th hours, 350 c.c.; total, 700 c.c. gastric juice.

It should be noted in this connection that Mr. V.'s noonday meal is in reality the big meal or dinner. He secretes less gastric juice on his evening meal, probably not more than 400 to 500 c.c., and from the fact that he makes his breakfast solely on biscuits, coffee, and milk it is likely that his secretion of gastric juice on the morning meal does not exceed 250 to 300 c.c. This would make a total of $I, 350$ to $I, 500$ c.c. of gastric juice secreted in 24 hours. These figures do not include the continuous secretion in the absence of food. It is of interest to note that Pflaunder arrived at practically the same figures ( 1,500 c.c. or 25 c.c. per $\mathrm{kg}$. of body weight in 24 hours), basing his estimate on calculations from the acidity and volume of the gastric content at varying periods after the meal.

- It need not be pointed out that the foregoing figures are subject to great variations, depending on the condition of the stomach and the quality and quantity of the food.

\section{SUMMARY}

The fluid contents of the "empty" stomach vary from nothing up to 150 c.c. The average of a number of tests varies with the individual from 30 to 50 c.c. The quantity is greater in the morning than at noon or at 6:00 P.M. It is on the whole greater in the summer than in the winter months. The most important factor in these daily and seasonal variations is probably the tonicity of the empty stomach and the rate of the continuous secretion.

The gastric glands in the normal person are never completely quiescent. The continuous secretion varies from 2 to 50 c.c. per hour. The higher figures are exceptional, but may obtain for several days in succession, again to revert to the lower figures. 
The vagus secretory tonus is a possible and the auto-digestion of the gastric juice itself is a probable factor in this continuous gastric secretion. The secretion itself is rich in pepsin, but when the secretion rate is very low it is poor in free hydrochloric acid.

Chewing on indifferent substances and stimulation of the nerveendings in the mouth by substances not related to food do not cause secretion of gastric juice, that is, these processes do not augment the continuous gastric secretion.

Seeing, smelling, and possibly thinking of palatable food usually cause a very slight and transitory secretion of gastric juice.

The rate of secretion of gastric juice on mastication of palatable food is directly proportional to the palatability of the food. During mastication the average rate is 3.5 c.c. per minute (minimum rate: I.4 c.c.; maximum rate: Io.8 c.c.). On. cessation of chewing the secretion rate diminishes rapidly, so that in $I_{5}$ to 20 minutes the gastric glands reach the level of the continuous gastric secretion. The chemistry of this appetite gastric juice is practically constant.

The latent period of this appetite secretion varies indirectly with the rate of the continuous secretion, so that when the continuous secretion is abundant the appetite secretion shows practically no latent period at all, while with the lowest rate of the continuous secretion the latent period varies from 2 to 3 minutes. This latent period is therefore one of the processes in the gland cells, and not in the nervous mechanism.

On the basis of these experiments on Mr. V., on the reports of other gastric fistula cases in man, and on the work of Pavlov on dogs, it is estimated that an adult normal person secretes on an average meal (dinner) 700 c.c. of gastric juice, or an average total of $\mathrm{I}, 500$ c.c. of gastric juice in 24 hours.

On the whole, this work on the appetite secretion of gastric juice in man confirms and extends the work of Pavlov and his pupils - on dogs. Pavlov overlooked or ignored the continuous secretion in the absence of all food and psychic stimuli, and he put too great an emphasis on the secretion induced in a hungry animal by seeing and smelling food. The significant appetite secretion in man is that induced by tasting and chewing good food. The 
continuous secretion does not fit in with Pavlov's general theory of strict adoption of the digestion juices to the food, as it apparently serves no useful purpose in digestion.

It is also clear that Pavlov overestimated the importance of the appetite secretion in gastric digestion. The continuous secretion initiates gastric digestion in the absence of appetite juice. Dogs with both vagi sectioned exhibit practically normal gastric digestion within a few days after the operation, despite the fact that the appetite gastric juice is eliminated. Cats may be forcibly fed with unpalatable food and the stomach digestion is practically as rapid as when they eat voluntarily. And we know that in man the pepsin-hydrochloric acid of the gastric juice may be greatly reduced if not entirely absent (achylia) without marked impairment of gastric peristalsis or food utilization. We have on numerous occasions removed all the appetite gastric juice from Mr. V.'s stomach before the masticated meal was put into his stomach without producing the slightest evidence of indigestion.

The significance of hunger and appetite for digestion is apparently not so much in the actual yield of appetite gastric juice as in the fact that when these sensation complexes are present the entire gastero-intestinal tract, both on the motor and on the secretory side, is in fit condition to handle the ingested food. 


\section{CHAPTER XV}

\section{THE CHEMISTRY OF HUMAN APPETITE GASTRIC JUICE}

\section{THE SOLIDS}

The total solids of the pure gastric juice of Mr. V. vary from $0.48 \mathrm{gm}$. to $0.5^{8} \mathrm{gm}$. per Ioo c.c., of which $0.34 \mathrm{gm}$. to $0.47 \mathrm{gm}$. is organic, and O.II gm. to o.I4 gm. inorganic material. The hydrochloric acid is, of course, expelled in the evaporation and drying of the gastric-juice residue.

The hunger gastric juice (continuous secretion) is distinctly higher than the appetite juice both in total and in organic solids.

The gastric juice or fluid in the empty stomach is distinctly more dilute than the appetite juice, although it may approach the concentration of the latter in cases where the rate of the continuous secretion is considerable.

The foregoing figures on Mr. V.'s appetite gastric juice are slightly higher than those given by Sommerfeld for the gastric juice from a ten-year-old girl, namely $0.40 \mathrm{gm}$. to $0.47 \mathrm{gm}$. Schmidt found in a human gastric-fistula case $0.58 \mathrm{gm}$. of total solids in the gastric juice, of which $0.32 \mathrm{gm}$. was organic, and $0.26 \mathrm{gm}$. inorganic. But Schmidt did not work with pure gastric juice. This is evident from his method of obtaining the juice, as well as from the fact that the acidity of the juice was only $0.20 \mathrm{gm}$. or less than half that of normal human gastric juice. Albu reports one experiment on a patient with hypersecretion finding the percentage of solids only$0.24 \mathrm{gm} .$, practically all of which (0.23 gm.) was inorganic salts. $\mathrm{He}$ also reports one determination on normal human gastric juice (pure appetite juice) in which the inorganic solids were $0.18 \mathrm{gm}$; the organic solids are not given.

Our results on $\mathrm{Mr}$. V. agree closely with most of those reported for the gastric juice of dogs. The total concentration of organic and inorganic substances is therefore about the same in the normal gastric juice (appetite juice) of man and dog. 


\section{SPECIFIC GRAVITY}

The specific gravity of Mr. V.'s appetite gastric juice varies between I,006 and I,009 with an average of 1,007 . This is the average of twenty tests on an equal number of gastric-juice samples. It will thus be seen that $\mathrm{I}, 009$ is an exceptional concentration.

The specific gravity of the continuous secretion is higher than that of the fluid or juice found in the empty stomach and lower than that of the appetite juice.

The specific gravity of the appetite gastric juice of the dog, as reported by Schoumow-Simanowski, Konovaloff, Friedenthal, and Rosemann varies from $\mathrm{I}, 002$ to $\mathrm{I}, 007$, with an average of $\mathrm{I}, 004$. This low average figure is probably due to the fact that in most of these experiments the gastric juice was collected for several hours after only a few minutes of sham feeding. There is evidence that the percentage of solids in the gastric juice is greatest during the first hours of appetite or digestion secretion. The concentration of the dog's appetite gastric juice during the first 20 minutes of secretion will in all probability be found identical with that of man for the same period.

\section{OSMOTIC CONCENTRATION}

The appetite gastric juice lowers the freezing-point from $-0.55^{\circ} \mathrm{C}$. to $-0.62^{\circ} \mathrm{C}$; the continuous secretion from $-0.47^{\circ} \mathrm{C}$. to $-0.52^{\circ} \mathrm{C}$; ; the fluid of the empty stomach from $-0.2 \mathrm{I}^{\circ} \mathrm{C}$. to $-0.4 \mathrm{I}^{\circ} \mathrm{C}$. The juice found in the empty stomach exhibits the greatest fluctuations in osmotic pressure, the appetite and the hunger juice being very constant. The hunger juice has, on the whole, a lower osmotic concentration than the appetite juice.

The foregoing figures for the appetite gastric juice of $\mathrm{Mr}$. V. are practically identical with those reported on the pure gastric juice of other human fistula cases. Sommerfeld (in a ten-year-old girl) found the freezing-point to vary from $-0.47^{\circ} \mathrm{C}$. to $-0.65^{\circ} \mathrm{C}$.; Kaznelson (twenty-five-year-old girl) reports a variation from $-0.46^{\circ} \mathrm{C}$. to $-0.54^{\circ} \mathrm{C}$. Umber reports two tests on the gastric juice (pure) of a fifty-nine-year-old man with cancer, finding a 
variation of $-0.15^{\circ} \mathrm{C}$. to $-0.82^{\circ} \mathrm{C}$. Assuming that Umber's determinations are correct, the gastric juice of this cancer patient was clearly not normal. We question whether the normal stomach can secrete a juice with an osmotic concentration so much greater than the blood as the figure $-0.82^{\circ} \mathrm{C}$. demands. The reader will note that the figures of Sommerfeld and Kaznelson, as well as our own for Mr. V., indicate an osmotic pressure of the appetite gastric juice not far below or above that of the human blood. According to Bickel the gastric juice (ten-year-old child) is always hypotonic to the blood. Lehman concludes that the osmotic pressure of normal gastric juice (gastric content) is usually less than $-0.50^{\circ} \mathrm{C}$., and that a concentration above this figure indicates hyperacidity or other pathological conditions. This view is obviously untenable.

The osmotic concentration of the dog's appetite gastric juice is practically identical with that of man. Sasaki reports a variation from $-0.5 \mathrm{I}^{\circ} \mathrm{C}$. to $-0.60^{\circ} \mathrm{C}$; ; Rosemann gives somewhat higher figures, or $-0.56^{\circ} \mathrm{C}$. to $-0.64^{\circ} \mathrm{C}$. On the other hand, Bickel reports extraordinary fluctuations in osmotic concentration of dogs' gastric juice (Pavlov pouch) or $-0.52^{\circ} \mathrm{C}$. to $-\mathrm{I} .2 \mathrm{I}^{\circ} \mathrm{C}$. We question whether the normal stomach can secrete a juice of the osmotic concentration $-\mathrm{I} .2 \mathrm{I}^{\circ} \mathrm{C}$., that is, twice that of the blood.

\section{TOTAL NITROGEN OF THE GASTRIC JUICE}

The total nitrogen was determined by the method of Kjeldahl on 9 different lots of appetite gastric juice of Mr. V. The average of all our determinations is $0.60 \mathrm{gm}$. nitrogen per roo c.c. appetite juice. The total nitrogen of the hunger juice was not determined. If all the nitrogen is in the form of proteins, and if we accept the figures of Nencki and Sieber, and of Pekelhaaring, namely, that nitrogen constitutes 14.39 per cent of the proteins of the gastric juice, the appetite gastric juice of man would contain on the average nearly $0.42 \mathrm{gm}$. protein per $100 \mathrm{c.c}$, or practically all the organic solids in the appetite juice.

Rosemann reports nitrogen determinations in 2 lots of dog's appetite gastric juice, finding $0.035 \mathrm{gm}$. and $0.054 \mathrm{gm}$. per Io० c.c., 
respectively. These figures are considerably lower than ours on the appetite juice of $\mathrm{Mr}$. V.

\section{AMMONIA}

The ammonia of the fresh gastric juice was determined by a combination of Folin's aeration and the Nessler colorimetric methods, using I to 5 c.c. of the juice. The ammonia cannot be determined by the Nessler reagent directly in pure gastric juice, as parallel tests on the same samples of gastric juice yield higher figures by aeration and Nessler than by Nessler direct (using I c.c. of the juice).

Ammonia in the amounts of 2 to $3 \mathrm{gm}$. per Ioo c.c. is a constant constituent of pure gastric juice of man and dog. The ammonia appears to be slightly more concentrated in the continuous secretion or hunger juice than in the appetite juice. The ammonia may' be greatly increased in gastric ulcers, and in certain normal persons the ammonia may also be exceptionally high ( 10 to I $5 \mathrm{mgr}$.).

Rosenheim and Strauss reported small amounts of ammonia in the gastric content of man. Zunz, working with the gastric content (test meals) on normal persons and on persons with various disorders of the alimentary tract, also reports the presence of ammonia. In the normal individuals the ammonia of the test-meal contents varied from 0.7 to $5.0 \mathrm{mgr}$. per 100 c.c. In cancers of the stomach the ammonia in the test-meal content was increased. The test meal introduces factors (bacterial action, saliva, etc.) not present in pure gastric juice. Sommerfeld, working with pure gastric juice of a ten-year-old girl with complete stricture of the esophagus, states that gastric juice contains no ammonia. Nencki, Zaleski, and Salaskin reported 4 to 5.5 mgr. ammonia per Ioo c.c. pure gastric juice of the dog. Rosemann reports the constant presence of a small amount of ammonia in the pure gastric juice (appetite secretion) in the dog. Reisner concludes that the ammonia in gastric juice comes from the saliva.

What is the origin and significance of the gastric-juice ammonia? It is known that saliva contains traces of ammonia. We find that the mixed saliva of man contains from 0.5 to $1.5 \mathrm{mgr}$. ammonia 
per 100 c.c. Salaskin found $2.5 \mathrm{gm}$. $\mathrm{NH}_{3}$ per roo c.c. in the saliva of the dog. But in dogs with Pavlov's stomach pouch, and in our gastric-fistula case, Mr. V., no saliva can enter the stomach or the part of the stomach yielding the juice.

The ammonia of the duodenal content may be a factor, as Boldyreff and others have shown the frequency with which intestinal content enters the stomach. This factor is excluded in dogs with the Pavlov stomach pouch. In our human fistula case this factor is readily controlled by making the ammonia determinations only on those samples of gastric juice that are absolutely free from admixture with bile, pancreatic juice, and succus entericus. Rosemann points out that the gastric-juice ammonia cannot be a simple filtrate from the blood since normal blood contains only about 0.5 mgr. of ammonia per roo c.c.

Huber, working in the author's laboratory, found that intravenous and oral administration of ammonia salts increases the ammonia concentration in the gastric juice. It is decreased on low, and increased on high protein diet. But when the urine ammonia is greatly decreased by taking alkalies, or greatly increased by taking acids, the concentration of the-gastric-juice ammonia remains unchanged.

The question of the origin of the gastric-juice ammonia is therefore very complex. (I) It is in part an active excretion from the blood. (2) It may be formed in part by deamidization of amino-acids in the gastric mucosa. (3) It may be formed in the process of secretion of gastric juice, or (4) by the action of the $\mathrm{HCl}$ on the gastric-juice protein, or on the cells of the mucosa. In . cases of gastric ulcers of infectious origin it may come in part from bacterial activity in the active focus of the ulcer.

In I898 Nencki, Pavlov, and Zaleski found that, per unit of mass, there is more ammonia in the gastric mucosa than in any other tissue of the body. These findings were essentially confirmed by Salaskin the same year. Huber found a greater concentration of ammonia in the fundic mucosa than in the mucosa of the cardiac and the pyloric ends of the stomach. These facts seem to indicate some relation of the ammonia formation to the secretion process 
itself and to the protein absorption, unless the higher ammonia content of the secreting and absorbing mucosa represents ammonium chloride in the process of absorption from the gastric juice.

\section{THE AMINO-ACIDS}

When deductions are made for the ammonia nitrogen of the juice, the formol-titrable nitrogen of Mr. V.'s appetite gastric juice varied from 3 to $9 \mathrm{gm}$. nitrogen per roo c.c. The gastric juice of a second fistula case (Mr. E.) gave $7 \mathrm{gm}$. per roo c.c. Four lots of dog's appetite gastric juice (Pavlov pouch) gave only I to $2 \mathrm{gm}$. of amino-acid nitrogen. It thus appears that normal human gastric juice contains slightly more amino-acid than ammonia nitrogen; but the greater part of the gastric-juice nitrogen is associated with more complex proteins.

Zunz reports that the amino-acid nitrogen (test meals) usually exceeds the ammonia nitrogen, and that both substances are increased in cases of gastric cancer. In three normal persons the maximum amino-acid was Io gm. per Ioo c.c. of gastric content, while in several gastric cancer cases it reached $\mathrm{I}_{5}$ to $20 \mathrm{gm}$. per Ioo c.c. of content. But these figures cannot be directly compared with ours on pure gastric juice, because of the uncertain factors associated with the gastric contents following a test meal.

\section{AUTO-DIGESTION OF THE GASTRIC JUICE}

When fresh gastric juice is incubated at $38^{\circ} \mathrm{C}$. the following changes take place in the proteins and the gastric mucin:

I. Practically all the ropy mucin and mucin flocculi are dissolved.

2. The pink color of the biuret reaction is increased. In fact, fresh human gastric juice gives practically a violet biuret reaction, and this color is intensified and changed toward pink by the auto-digestion.

3. The characteristic protein precipitation at the point of neutralization is decreased.

4. The quantity of proteins precipitated by nitric acid and by heat is reduced. 
It is thus clear that the protein of pure gastric juice undergoes pepsin-hydrochloric acid digestion in the stomach itself. But some of the gastric juice proteins are not hydrolyzed, at least not down to the peptone stage. It is an interesting fact that the protein of the gastric juice of man injected into guinea-pigs sensitizes the animals to subsequent injections of human serum, but injections of even large quantities of human gastric juice into pigs thus sensitized produce no anaphylaxis. This auto-digestion of the gastric juice itself is probably a factor in the continuous secretion of gastric juice in the way of yielding gastric secretagogues.

\section{ACIDITY OF NORMAL GASTRIC JUICE}

The acidity was determined by titration with $\mathrm{N} / 40 \mathrm{NaOH}$, and using dimethyl-amino-azo-benzene and phenothalein as indicators for the free and the total acidity, respectively. During the 4 years that Mr. V. has been under observation hundreds of determinations have been made of the acidity of the contents of the "empty" stomach, of the hunger juice, or continuous secretion, and of pure appetite juice. The reader will recall that the contents of the "empty" stomach are taken one hour after washing out the stomach with 200 c.c. of water. All the cases where the gastric juice or gastric content was contaminated with bile (intestinal content) are excluded from the summaries given in Table IV.

The second gastric-fistula case, Mr. E., was a man of twenty-six years of age, healthy and vigorous. Nearly a year prior to our work on him his esophagus was corroded with a solution of lye, and this led to a nearly complete cicatricial stenosis, and hence the gastrostomy. At the time of the observations the esophagus had been dilated sufficiently to permit swallowing of any wellmasticated food, and the gastrostomy opening was used only in the dilation processes. In this case, saliva is therefore not excluded from the contents of the empty stomach, and possibly not from the continuous or hunger secretion, although Mr. E. was instructed and urged not to swallow any saliva during these experiments. The appetite juice was obtained by Mr. E.'s chewing palatable 
food, and spitting out the chewed food, care being taken not to swallow saliva or particles of food.

These results from my two gastric-fistula cases are in agreement with the work of Pavlov and his pupils on dogs, and the work of previous observers on pure gastric juice of normal persons. The latter data have recently been brought together and discussed by Boldyreff.

\section{TABLE IV}

Acidity of Normal Human Gastric Juice

\begin{tabular}{|c|c|c|c|c|c|c|c|c|}
\hline \multirow{2}{*}{ - Person } & \multirow{2}{*}{ Material } & \multirow{2}{*}{$\begin{array}{l}\text { No. OF } \\
\text { OBSER- } \\
\text { VATIONS }\end{array}$} & \multicolumn{6}{|c|}{ Acidity } \\
\hline & & & \multicolumn{3}{|c|}{ Free } & \multicolumn{3}{|c|}{ Total } \\
\hline - & & & Low & High & Aver. & Low & High & Aver. \\
\hline \multirow{3}{*}{ Mr. V... } & Cont. empty stomach. & 235 & 0.10 & 0.35 & O. 18 & O. I5 & 0.40 & 0.23 \\
\hline & Hunger juice. . . . . . & I80 & O. I5 & 0.35 & 0.25 & 0.20 & 0.45 & 0.34 \\
\hline & Appetite juice. & 285 & 0.35 & 0.44 & 0.40 & 0.40 & 0.53 & 0.48 \\
\hline \multirow{3}{*}{ Mr. E... } & Cont. empty stomach. & Io & $0: 09$ & 0.36 & 0.20 & 0.18 & $0.4 \mathrm{I}$ & 0.25 \\
\hline & $\{$ Hunger juice. . . . . . . & 8 & 0.20 & 0.32 & 0.25 & 0.27 & 0.38 & 0.33 \\
\hline & Appetite juice...... & I 5 & 0.30 & $0.3^{6}$ & 0.34 & 0.36 & 0.47 & 0.44 \\
\hline
\end{tabular}

Normal human gastric juice (appetite secretion) when secreted above a certain minimum rate shows a practically constant total acidity of nearly 0.5 per cent $\mathrm{HCl}$, or the same as the gastric juice of normal dogs. Practically the same figure is obtained when the acidity is determined by conductivity methods (Menten). The gastric juice (appetite as well as hunger juice) secreted by the normal stomach at a low rate shows lower than normal acidity and total chlorides. The view of Pavlov based on experiments on dogs that gastric juice is secreted at uniform and constant acidity is true for man only in regard to appetite, digestion, and hunger juice secreted at fairly high rate. We must take cognizance of the equally important fact that the normal gastric mucosa is capable of secreting a juice of submaximal acidity.

The studies of Rehfus and Hawk on the acidity of the gastric content at varying periods after drinking water and ingesting an 
Ewald test meal also support the view that the normal gastric juice has a fairly constant acidity.

The reader will note that normal human gastric juice is equal in total acidity to the maximum acidity reported by clinical observers for so-called hyperacidity in man. So far as we are acquainted with the literature, there is no evidence that the gastric glands under any pathological conditions are able to or do secrete a juice of higher than normal acidity. Actual hyperacidity does not occur in cases of experimental ulcers in the stomach and duodenum. But there may be hypersecretion. Moreover, the presence in the stomach of gastric juice of full acid strength leads by itself and immediately to no untoward symptoms.

The contents of the "empty" stomach, and the continuous or hunger secretion (when the secretion rate is low) have uniformly a lower acidity than the appetite juice. The total acidity of contents of the "empty" stomach is 0.2 or less. The reader will note that this figure is frequently given as the acidity of pure gastric juice of normal persons. The acidity of the continuous or hunger secretion is higher, and the greater the secretion rate the higher the acidity until it may equal that of the appetite juice. In no instance does the acidity of the continuous secretion exceed that of the appetite juice.

What is the cause of the low acidity of the continuous secretion and contents of the empty stomach? The following factors must be taken into account:

I. The actual acidity of the juice as secreted may increase with the secretion rate, until the maximum acidity is reached with the high average rate of secretion, under conditions similar to those obtaining in the case of the salivary glands where the concentration of the salts and the organic materials increases with the rate of salivary secretion. If this is a factor the gastric juice secreted at a low rate should show a lower osmotic concentration and a smaller total of chlorides than the juice secreted at high rate. The figures reported by Umber for man and by Rosemann for the dog support this view, the former investigator showing particularly that the osmotic concentration of the gastric juice increases with the rate 
of secretion. The cryoscopic data may, however, be misleading, as the salts produced by the neutralization of the $\mathrm{HCl}$ may not dissociate as freely as the acid.

2. The slower rate of secretion may give a chance for the $\mathrm{HCl}$ to be partly neutralized by the alkaline mucus secreted by the mucin cells of the gastric mucosa. This is the factor emphasized by Pavlov. In fact, Pavlov takes the position that in the normal animal gastric juice has practically a constant acidity, irrespective of the secretion rate, but the actual acidity of the juice in the cavity of the stomach is purely a matter of rate of neutralization. If this is the sole factor, the total chlorides of the gastric juice ought to show a greater constancy than the acidity. That the hydrochloric acid of the gastric juice is in part neutralized by the gastric mucus is obvious. But according to Boldyreff the alkalinity of gastric mucus is only 0.05 to $0.10 \mathrm{Na}_{2} \mathrm{CO}_{3}$. That is to say, it would require roo to 200 c.c. of gastric mucus to reduce roo c.c. of gastric juice from the normal acidity of 0.45 down to 0.25 by neutralization and dilution. The importance of this factor has therefore been overestimated by Pavlov.

3. When the gastric juice is collected from a Pavlov accessory stomach, or from an individual with complete closure of the esophagus, as is the case with Mr. V., the saliva cannot be a factor in lowering the gastric juice acidity by neutralization and dilution. When all or most of the saliva is swallowed the acidity of the gastric juice is necessarily reduced in proportion to the relative rate of salivary and gastric secretion. This is effected by dilution rather than by neutralization, as the titration alkalinity of saliva is low ( $0.8 \mathrm{Na}_{2} \mathrm{CO}_{3}$; Neumeister, cited by Boldyreff).

According to Boldyreff, Carlson, Hicks and Visher, Rehfus and Hawk, an important factor in lowering the acidity of gastric juice from that actually secreted by the gland (0.5) to that usually found in the cavity of the stomach (0.25), is the entrance of the alkaline intestinal contents (pancreatic juice, bile, and succus entericus) into the stomach. This usually occurs, probably, when the acid in the stomach mounts much above 0.25 . This "mechanism for self-regulation of the acidity of the stomach content" 
probably breaks down in cases of so-called "hyperacidity" in man. In cases of "hypersecretion" the quantity of juice secreted is greater than normal, and the secretion may persist in the absence of all normal stimuli, but the neutralizing factors suffice to reduce the acidity of the juice approximately to that found in the normal stomach. It is purely a balance of secretion rate and of neutralization capacity. Impairment of the neutralization factors or a very excessive secretion rate of gastric juice, or pyloric obstruction would tend to render the acidity of the gastric content equal to that of pure gastric juice; in other words, produce clinical "hyperacidity."

\section{TOTAL CHLORIDES}

The total chlorides of the appetite gastric juice of $\mathrm{Mr}$. V. are very constant, the minimum being 0.49 per cent and the maximum 0.56 per cent chlorine. The continuous secretion or hunger juice is more variable in chloride content, and this variation appears to be directly dependent on the secretion rate and on the acidity. In general, the lower the secretion rate the lower are the acidity and the total chlorides. This is in agreement with the findings of Foster and Lambert on dogs.

These facts seem to point to the conclusion that the low acidity of the gastric juice secreted at a slow rate is not due entirely to neutralization. We have apparently a secretion of gastric juice of an acidity actually lower than that of the rapidly secreted appetite juice. The dependence of the actual secreted acidity on the secretion rate is not a very close one, however, as we may have very marked fluctuation in rate without any change in chlorides. But below a certain secretion rate ( 25 to 30 c.c. per hour from the entire stomach of the adult) an actual hypoacid juice is secreted.

The foregoing figures for total chlorides in the normal gastric juice of Mr. V. agree closely with the findings of previous observers on the gastric juice of dog and of man. Rosemann gives 0.54 to $0.64 \mathrm{Cl}$ for the appetite gastric juice of the dog. The figures given by Sommerfeld for human appetite juice vary from 0.53 to $0.59 \mathrm{Cl}$. Umber, working on an old man (fifty-nine years) with partial eso- 
phageal stenosis (malignant), reports total chlorides of the gastric , juice as varying from 0.27 to $0.60 \mathrm{Cl}$.

\section{CONCENTRATION OF PEPSIN}

In our tests the digestion mixture was made up of I c.c. gastric juice and 5 c.c. $\mathrm{N} /$ Io $\mathrm{HCl}$. The egg albumin in the Metts tubes was coagulated boiling water for ro minutes. The digestion time was 24 hours at $37^{\circ} \mathrm{C}$. Under these conditions the pepsin concentration of the gastric juice of $\mathrm{Mr}$. V. showed the following figures: appetite juice, $6 \frac{1}{2}$ to $7 \frac{1}{2} \mathrm{~mm}$. digestion; continuous secretion, 6 to $7 \mathrm{~mm}$. digestion; contents of empty stomach, 3 to $4 \mathrm{~mm}$. digestion. The results are stated in the length of albumin column actually digested, because, according to Cobb, the law of Schütz does not hold for pepsin in concentrations that digest more than 4 to $5 \mathrm{~mm}$. in 24 hours.

The appetite gastric juice of Mr. E., our second gastric-fistula case, when tested as above in $\mathrm{I}_{4}$ experiments, showed a pepsin concentration of 5 to $7 \mathrm{~mm}$. with an average of $6 \mathrm{~mm}$., a slightly lower value than the gastric juice of $\mathrm{Mr}$. V. The pepsin concentration of the gastric juice of normal dogs runs somewhat lower, or 2 to $5 \mathrm{~mm}$.

When the Metts tubes are placed in 16 c.c. of undiluted human gastric juice (appetite secretion) the digestion in 24 hours at $37^{\circ} \mathrm{C}$. varies from $\mathrm{I} 2$ to $\mathrm{I} 6 \mathrm{~mm}$., or only twice the quantity digested in the dilution of I c.c. juice to 15 c.c. $N /$ Io $\mathrm{HCl}$. This seems to indicate that in normal gastric juice the pepsin is present in excess of the needs or at least far in excess of that needed in economic digestion.

The U.S. Pharmacopeia defines "Ioo per cent pepsin as a preparation capable of digesting three thousand times its own weight of finely divided egg white (coagulated) in three hours." The Pharmacopeia test is carried out as follows: Io gm. of boiled white of egg is macerated through a No. 40 filter and placed in 40 c.c. 0.3 per cent $\mathrm{HCl}$., $3^{\frac{1}{3}} \mathrm{mgr}$. dried pepsin added, and the mixture incubated at $52^{\circ} \mathrm{C}$. for 3 hours, with occasional stirring. After being treated in this manner, there is only a very small residue 
of undissolved egg white at the end of three hours, but the procedure of measuring the amount of this residue does not yield very accurate results.

This test was applied to six different lots of appetite gastric juice of Mr. V. Under the foregoing conditions, I to $\mathrm{I} \frac{1}{2}$ c.c. appetite gastric juice digested ro gm. of coagulated and finely divided egg white in 3 hours practically as completely as is done by $3 \frac{1}{3} \mathrm{mgr}$. "roo per cent pepsin." As defined by the U.S. Pharmacopeia; I c.c. of human gastric juice must therefore contain $3 \frac{1}{2} \mathrm{mgr}$. pepsin, or Ioo c.c. of the juice, $35 \mathrm{mgr}$. pepsin. We have seen that the appetite gastric juice of man contains about $400 \mathrm{mgr}$. organic material per Ioo c.c. That is, according to the Pharmacopeia definition, only about ro per cent of the organic matter in the human gastric juice is pepsin.

It has been shown that an adult normal person, if hungry, secretes 600 to 700 c.c. gastric juice after an average palatable dinner, or a total of about I,500 c.c. gastric juice in 24 hours. That is to say, there is a secretion of 240 to $250 \mathrm{mgr}$. pepsin per dinner, capable under proper conditions of digesting from $630 \mathrm{gm}$. to $75^{\circ}$ gm. of protein (coagulated and finely divided egg albumin) in 3 hours; and the total pepsin secretion in 24 hours is $525 \mathrm{mgr}$. capable of digesting $\mathrm{I} \frac{1}{2} \mathrm{~kg}$. proteins (coagulated egg white) in 3 hours.

It is therefore clear that the normal human stomach secretes pepsin far in excess of the actual needs of gastric digestion, or, more precisely, far in excess of what can be used advantageously under ordinary conditions of gastric digestion. When the boiled egg white is broken up in larger pieces, such as occurs in ordinary rapid mastication, I c.c. of gastric juice requires 6 to ro hours for complete digestion.

This great excess of pepsin in normal gastric juice probably explains the clinical findings of great reduction in pepsin content without any evidence of impaired gastric digestion. It probably also explains, in part at least, the practical uselessness of commercial pepsin as a therapeutic measure in gastric disorders. 


\section{CHAP'TER XVI}

\section{HUNGER AND APPETITE IN DISEASE}

\section{ANALYSIS OF THE PROBLEM}

We believe all physicians will agree that the control of hunger and appetite is a very important factor in the control of disease, and especially of chronic disorders. The physiologist is therefore assured of the co-operation of the clinics in the investigation of the pathology of hunger and appetite. Such co-operation is a sine qua non for progress in this field, as the final verdict in the analysis of the pathology of hunger is the word and the reactions of the patient himself.

Conditions of disease in man, so far as they affect hunger, usually involve a decrease or absence of hunger and appetite sensations, as in fevers, anemias, cachexias, and neuroses of various origin. Certain pathological states such as diabetes, brain tumors, neuroses, etc., may be associated with abnormally strong hunger and appetite. In diseases of the stomach itself the hunger sensation may be decreased (atony, gastritis, constipation, etc.), increased (hypermotility, pyloric insufficiency, vagotonia, etc.), or altered in the direction of abnormal painfulness, as in gastric and duodenal ulcers.

From our analysis of the nature of the hunger mechanism, it is evident that depression or absence of the hunger sensation may theoretically be brought about in any one of the following ways: (I) direct failure or absence of the tonus and hunger contractions of the empty stomach; (2) prolonged reflex inhibition of the stomach; (3) interference with or depression of the central conduction paths; (4) direct depression of the cerebral or thalamic hunger centers; (5) interference with the central conduction of the hunger impulses by abnormal or unusually strong impulses from other proprioceptors. These various conditions may be caused by pathological changes in the blood, in the motor 
mechanism of the stomach itself, or in the central conduction paths and sensory centers. Pathological augmentation of hunger would result from the reversal of these conditions. The first question that must be settled in the physiological pathology of hunger is whether or not the abnormalities are due to changes in the gastric hunger mechanism itself. This can in every case be settled by direct tests on the patient, using our balloon method. When the pathological changes are in the central nervous system itself their analysis becomes much more difficult.

II. THE LITERATURE ON HUNGER AND APPETITE IN DISEASE IN MAN

I. Bulimia.-Abnormally intense hunger sensation has been termed bulimia. Some authors do, others do not, distinguish between bulimia and polyphagia. When distinctions are made, the term polyphagia is used to denote the condition of excessive ingestion of food, that is, an absence of satiety or sensation of fulness, rather than an abnormally intense hunger sensation. Bulimia may be temporary or chronic. It is characterized by the fact that hunger comes on shortly after eating, and if it is not appeased by food there follow headache, weakness, and prostration, just as in normal hunger in many persons, only to a much greater degree. Very small quantities of food may appease this hunger temporarily. This sensation of hunger may arise before the stomach is empty, although in many cases of bulimia, the food leaves the stomach more rapidly than in normal persons (Ewald, Perthes, Sick, Leo, Boas). Nicolai concluded that bulimia is due to hyperexcitability of the afferent hunger nerves in the stomach and esophagus. Ploenius insists that bulimia is an augmentation of normal hunger, and appears only when the stomach is empty; and that it invariably indicates organic lesions in the stomach, such as local destruction of the mucosa with pepsin- $\mathrm{HCl}$ corrosion of the deep tissues. According to Leo, bulimia may occur in exophthalmic goitre, in gastric and duodenal ulcer, with hyperacidity, in chronic gastritis, diarrhoea, tapeworms, pregnancy, excessive menstruation (hemorrhage), and even in cancer and in dilation of 
the stomach. Ewald records bulimia associated with Addison's disease, syphilis, uterine diseases, and brain injuries (tumors, emboli, trauma). Meyer states that excessive hunger or bulimia may appear in all types of neurosis.

This hunger has usually a very sudden onset, and is satisfied or even turned into nausea by very small quantities of food. Meyer describes another type of intense hunger accompanied by headache, in which neither the hunger nor the headache is relieved by eating, and the ingestion of food does not lead to the sensation of fulness or satiety. But in these patients there was evidence of other cerebral disturbances. Meyer argues that in cases where the intense hunger is satisfied or even turned to nausea with a few mouthfuls of food we cannot be dealing with the mere augmentation of true hunger. This position is not tenable. There may be increased excitability of the inhibitory reflexes from the mouth and the gastric mucosa, and hyperexcitability of the mucosa nerveendings would lead to nausea after ingestion of even small quantities of food. Meyer also reports excessive hunger in certain persons past middle life with tendency to adiposity. Perthes reports bulimia in persons with patent pylorus. In some of Perthes's patients the hunger was intense enough to wake them up from sleep every two hours during the night. Boas assumes a type of "idiopathic" bulimia, not accompanied by" any other functional or organic disturbances.

The excessive hunger in pregnancy is in all probability a normal physiological effect of the increased metabolism due to the growing fetus. The peculiar fluctuation in the appetite or desire for certain kinds of food that may occur in pregnancy involves more complex factors.

The bulimia of hypochondriacs, and other types of neurotics, may be a subjective exaggeration of normal hunger impulses.

2. "Hunger pain."- The gastric pains that appear in the empty stomach or a few hours after ingestion of a meal in persons with gastric or duodenal ulcers, or with gall-bladder disease are designated by Moynihan, Hertz, and others as "hunger pains." In normal persons the hunger sensation, if sufficiently strong, is painful. 
The patients with ulcer or hypersecretion describe these hunger pains in part as continuous or persistent, in part as variable or "gnawing," precisely like the normal pangs of hunger, except that they are more intense or painful. Like the normal pangs of hunger the pathological hunger pains are allayed by the ingestion of food. Most of the German authors do not differentiate between bulimia and the hunger pains of gastric and duodenal ulcers.

Following Moynihan, many clinicians regard the hunger pains in ulcers as caused by hydrochloric acid stimulation of the raw surfaces of the ulcer. In support of this view they cite the fact that the pains are temporarily relieved by giving alkalies by mouth. Hertz has shown, however, that giving strong acids by mouth to ulcer patients does not cause or increase the hunger pains. If the acid stimulation of the ulcer gives rise to these hunger pains in ulcer patients, they have a different origin from the normal hunger pains, as the latter are caused by strong contractions of the stomach, especially of the fundic and cardiac regions. The gnawing or rhythmical character of the hunger pains in ulcer cases appears to the author to show that they are due to contractions. If they were caused by chemical stimulation of the sensory nerves directly we should expect them to be continuous. Hertz has pointed out additional facts that support the mechanical or contraction origin of the pains, such as the patency of the pylorus, the rapid emptying of the stomach, the hypertonicity and hyperperistalsis of the stomach, etc., in both duodenal and gastric ulcer. Moreover, hypersecretion and hyperacidity is not a constant factor in ulcers (Hardt), and the hunger pains in ulcers may closely simulate the gastric pain in cases of gallstones, appendicitis, gasteroptosis, etc., where there is no raw mucous surface to be stimulated chemically, either in the stomach or the duodenum.

Edelmann states that the gastric digestion peristalsis is directly dependent on the acidity of the gastric juice. But according to Elsner the condition of achylia gastrica in man has per se no effect on the digestion movements. The emptying time of the stomach in achylia may be normal, less than normal, or greater than normal. 
'The recent studies on man by Spencer, Meyer, Rehfus, and Hawk have led them to conclude that weak alkalies (sodium bicarbonate) in the stomach hasten the emptying of the stomach by increasing the digestion peristalsis or by opening the pylorus. This is opposed to the generally accepted view of the acid control of the pylorus, as developed by Cannon. If their conclusion is substantiated, we may have the explanation of the allaying of the "hunger pains" in ulcer patients by oral administration of alkalies. That is, the alkalies cause relaxation of the tetanic spasm of the pylorus and antrum, induced reflexly from the duodenum. Glassner and Kreuzfuchs state that in cases of gastric ulcer there is a prolonged tetanic closure of the pylorus when the acid chyme first enters the duodenum. This is a reflex from the duodenum. The body of the stomach is atonic and quiescent during this pyloric spasm. The spasm itself is felt as pain. In cases of duodenal ulcers similar pylorus spasms come on later in digestion and are likewise felt as pains. According to these observers the hunger pains in ulcers are thus due to pylorus spasm, while Hertz ascribes them to contractions of the entire antrum. Pick suggests that the pains are due to mechanical stimulation of the food rather than to the acid stimulation of the ulcer. Ehrlich protests against the view that "painful empty stomach" in ulcer patients is an evidence of neurosis. According to Jacobi, the pains of gastric and duodenal ulcers are due to "hypersecretion, hyperperistalsis, or pressure."

Are the sensations of "hunger pains" identical with the normal pangs of hunger except for their greater degree of painfulness? This question cannot be answered from the clinical literature, and probably will remain unanswered until the clinical investigator himself experiences the pain in ulcer and allied diseases. These pains may lead to or be associated with nausea, but the same is true in normal hunger of some persons and in prolonged starvation. The "peristaltic unrest" of Kussmaul occurs both in the filled and in the empty stomach, in cases of pyloric obstruction and in certain forms of neurosis. The sensations aroused by this condition are said to be similar to cramps rather than to the pangs of hunger. The gastralgokenosis of Boas is thought by this author to be due 
to the strong contractions of the pyloric part of the stomach toward. the end of gastric digestion. Although the pain disappears on taking food, Boas does not identify it with true hunger pains. The gastralgia in tabes is generally held to be of central origin, but so far as we know the tonus and motor conditions of the stomach have not been studied during the gastric crisis of this disease.

3. Polyphagia and akoria.-The term "polyphagia" is frequently used in the sense of bulimia or excessive hunger. Other authors confine it to excessive ingestion of food without actual augmentation of the hunger sensation. In this latter sense it implies essentially delay in or absence of the sensation of satiety. Nicolai believed that polyphagia results from anesthesia of the stomach nerves concerned in the sensation of satiety. But true akoria or absence of satiety occurs mainly in hysteria and neurasthenia, and has probably nothing in common with such a condition as the polyphagia in diabetes.

4. Anorexia.-The diminution or absence of hunger and appetite in gastero-enteritis, in fevers, and in cachexia, etc., are in all probability associated with atony of the stomach and absence of the gastric hunger contractions. The evidence for this view will be presented later. The true anorexia nervosa is probably of more complex origin, involving both central and peripheral factors. There may be atony and absence of the gastric hunger contractions even in cases where no organic lesion in the stomach can be demonstrated. In some cases of anorexia Ewald found pathological changes in the mucous membrane of the mouth, suggesting disorders of the sense of taste. .

When the anorexia is very marked it may be accompanied by nausea, at least at the sight, smell, or taste of food, or an actual fear of food and eating (sitophobia). Under other conditions a mild nausea may be present synchronously with practically normal hunger and appetite (Boas). We have seen that this may occur in normal individuals in prolonged starvation. Nervous anorexia is more common in women than in men. Vertes takes the position that most of the disturbances of hunger and appetite that appear in pregnancy, ovarian diseases, menstrual disorders, etc., are in 
reality independent of the latter, while in a few cases, they are reflex neuroses. The actual state of the gastric hunger mechanism in these conditions is not known.

5. Parorexia.-This term is used to designate various types of abnormal, perverted, or depraved appetite. These conditions refer exclusively to appetite, rather than to hunger, at least it has not been shown that hunger is at all involved. The least abnormal condition appears to be the malacia, or desire for highly spiced or acid foods that are sometimes seen in chlorotic girls and in pregnant women. Another type-pica-is the appetite for substances that are not food, such as clay, chalk, or earth. These materials are eaten, especially by children, because they like the taste of them. This is probably a bad habit, rather than an indication of seriously perverted mental processes. Most of our domestic animals, even those that appear perfectly healthy, eat earth at times, aside from the quantity of earth consumed with their ordinary food and drink. And even with the best of care in the way of food hygiene, we all consume more or less earth, dirt, stable manure, etc., with our food. To the mind of the average adult earth has become synonymous with dirt or filth, hence we abhor taking it into the mouth, just as the average person loathes snakes. The child, not having formed these associations, puts sand, chalk, or earth into his mouth from general curiosity or in the spirit of play, and may or may not like the new experience. If he likes it, he will repeat it until he learns the usual social canons. Clay eating is on a par with gum chewing and tobacco chewing, and is certainly less injurious and nasty than the latter habit.

6. Allotriophagia, or desire for disgusting and offensive subtances, such as human excreta, body lice, etc., is practically confined to insane individuals, or extreme degenerates. It is likely that the habit is reached via the route of sexual depravity, at least in many cases. In persons with the ordinary idea-associations lost or suppressed, tolerance and even liking for any kind of taste and odor can apparently be cultivated. We all know that animal excreta are regularly ingested by many normal animals, though most of them pass by those of their own species. 
III. CONDITION OF THE GASTRIC HUNGER MECHANISM IN DISEASE OF EXPERIMENTAL ANIMALS

I. Pancreatic diabetes.-Experimental pancreatic diabetes in animals is accompanied by the excessive hunger or polyphagia of diabetes melitus in man. Dr. Luckhardt studied the gastric hunger contractions in two dogs during the entire course of fatal pancreatic diabetes. With good care dogs live from 4 to 6 weeks after complete pancreatectomy, showing progressive emaciation despite their excessive intake of food. In both the diabetic dogs the gastric tonus and hunger contractions were more continuous and vigorous than in normal dogs or in the same dogs before they became diabetic. This augmentation of the gastric hunger contractions persisted up till within 24 hours of the death of the animals, despite the progressive and finally extreme emaciation and weakness. The increased food consumption of the dogs thus ran parallel with the greater vigor of the peripheral hunger mechanism. And there can be little doubt that the dogs felt greater hunger and consumed more food because of the greater vigor of the gastric hunger contractions.

The cause of this augmented contraction of the empty stomach in diabetes is still an open question. It is evidently due, at least in part, to some change in the blood, for transfusion of diabetic blood into normal dogs stimulates the hunger contractions in the latter. Allen states that when partial diabetes in man is controlled by means of temporary starvation and dieting, so that the urine becomes free from sugar and the acidosis disappears, the polyphagia of the diabetic patient also disappears, but we do not know whether the latter is due to a return of the gastric hunger mechanism to the normal state of activity.

2. Polyphagia and augmented gastric hunger contractions in dogs with mange.-A number of dogs kept in the laboratory for long periods in the course of certain lines of investigation became afflicted with mange. Dr. Luckhardt observed that these mangy dogs consumed a much greater amount of food than they did before contracting or after being cured of the disease. It was also noted that a dog with mange is more susceptible to cold than normal dogs, as shown by their almost constant shivering, even in a room 

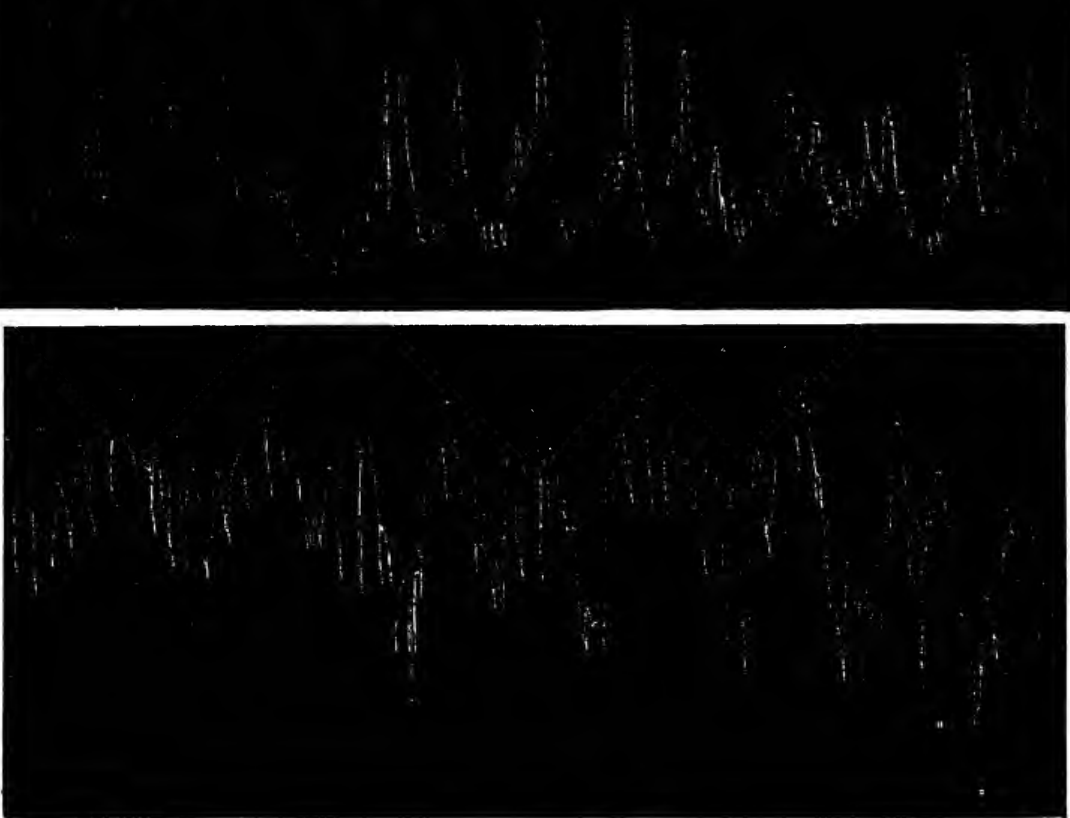

Fig. 30.-Records of the gastric hunger contractions of a dog before and during fatal pancreatic diabetes (Luckhardt). Bottom of tracings to pressure of bromoform. $A$, type II contractions indicating moderate hunger in dog before rendered diabetic. Four-fifths original size. $B$, culmination of a tetany period in the diabetic animal lasting about 20 minutes. Smaller tetany periods are likewise shown. Throughout, a type III rhythm on a high tonus. 'Two-thirds original size. $C$, tracing obtained from the empty stomach of a diabetic dog less than 2 days before death. Throughout, type III contractions on a high tonus. Dog too weak to walk. Ate $105 \mathrm{gm}$. meatthe last meal before death, which followed 2 days later. 'Two-thirds original size. 
270 CON'TROL OF HUNGER IN HEAL'TH AND DISEASE

with temperature ranging from $55^{\circ}$ to $65^{\circ} \mathrm{F}$. This is probably due to the loss of hair, and to interference with the normal vasoconstrictor reflexes of the skin by the persistent cutaneous
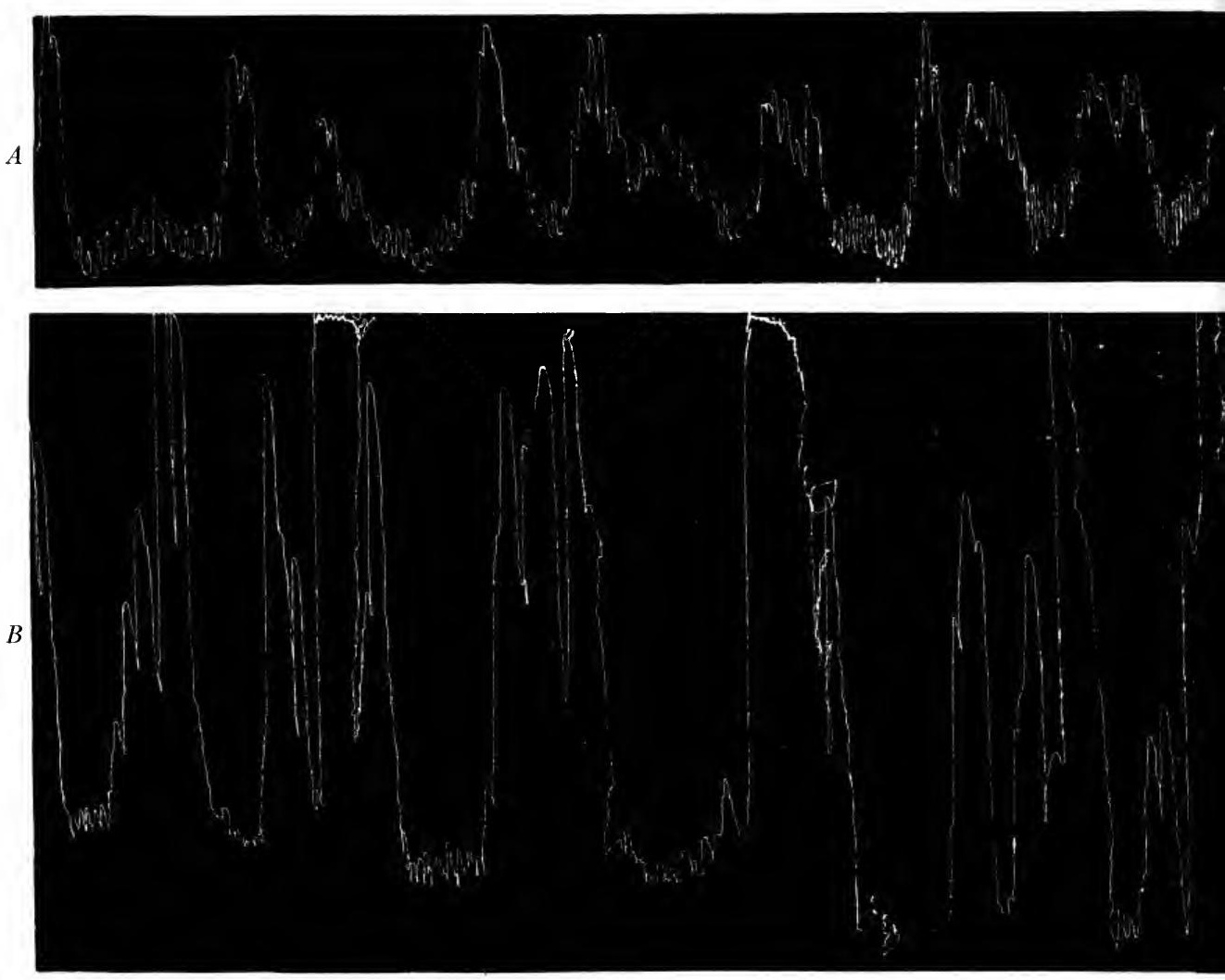

FIG. 31.-Tracing from empty stomach of dog while normal $(A)$, and after developing severe mange $(B)$; showing abnormally intense gastric hunger parallel with the mange and the polyphagia (Luckhardt).

hyperemia. These conditions must involve an abnormal heat loss from the skin.

The gastric tonus and hunger contractions were studied in a number of these mangy and polyphagic dogs, in order to determine whether the greater hunger was due to greater gastric hunger 
contractions. 'This proved to be the case. A dog with extensive mange but otherwise healthy exhibits abnormally great gastric tonus and hunger contractions. The latter tend to become tetanic, and when this is not the case, the individual contractions are abnormally strong. The polyphagia of the mangy dogs is therefore due to the greater vigor of the gastric hunger mechanism. 'The stronger gastric contractions are probably brought about indirectly through increase in muscular metabolism as a result of the too great loss of heat from the skin, in other words, the same effects as we found on exposing normal men and animals to great and prolonged cold.

3. Hunger and appetite in cases of alcoholic gastritis in dogs.Dr. Luckhardt found that whiskey or strong alcohol introduced directly into the empty stomach of dogs in sufficient amount to induce marked narcosis abolishes the tonus and hunger contractions of the stomach for 24 to 36 hours. During this period the animal refuses food and may vomit at times. After 36 to 48 hours the hunger contractions gradually return and may at times even approach the condition of incomplete tetanus. The dog shows interest in food, but may eat only a mouthful. Occasional vomiting still continues. Evidently the gastric mucosa continues hypersensitive for a much longer time than the period of motor paralysis of the stomach, so that ingestion of food induces or increases nausea and gastric distress, despite the fact that hunger contractions and hunger sensations are present. In other words, the dog experiences hunger and nausea at the same time. That the dog actually feels the contractions of the empty stomach as hunger seems to be shown by the fact that he walks up to the food from time to time, sniffs at the food, and may even start to eat a little. At the end of several days, vomiting disappears entirely and the dog resumes normal feeding.

The interesting point in these observations is the recovery of strong gastric hunger contractions a considerable time before the dog starts normal feeding. We think the disinclination to eat, despite moderately strong hunger, is due to nausea induced from the hyperexcitable gastric mucosa. After-effects of alcohol on the brain may also play a rôle. 

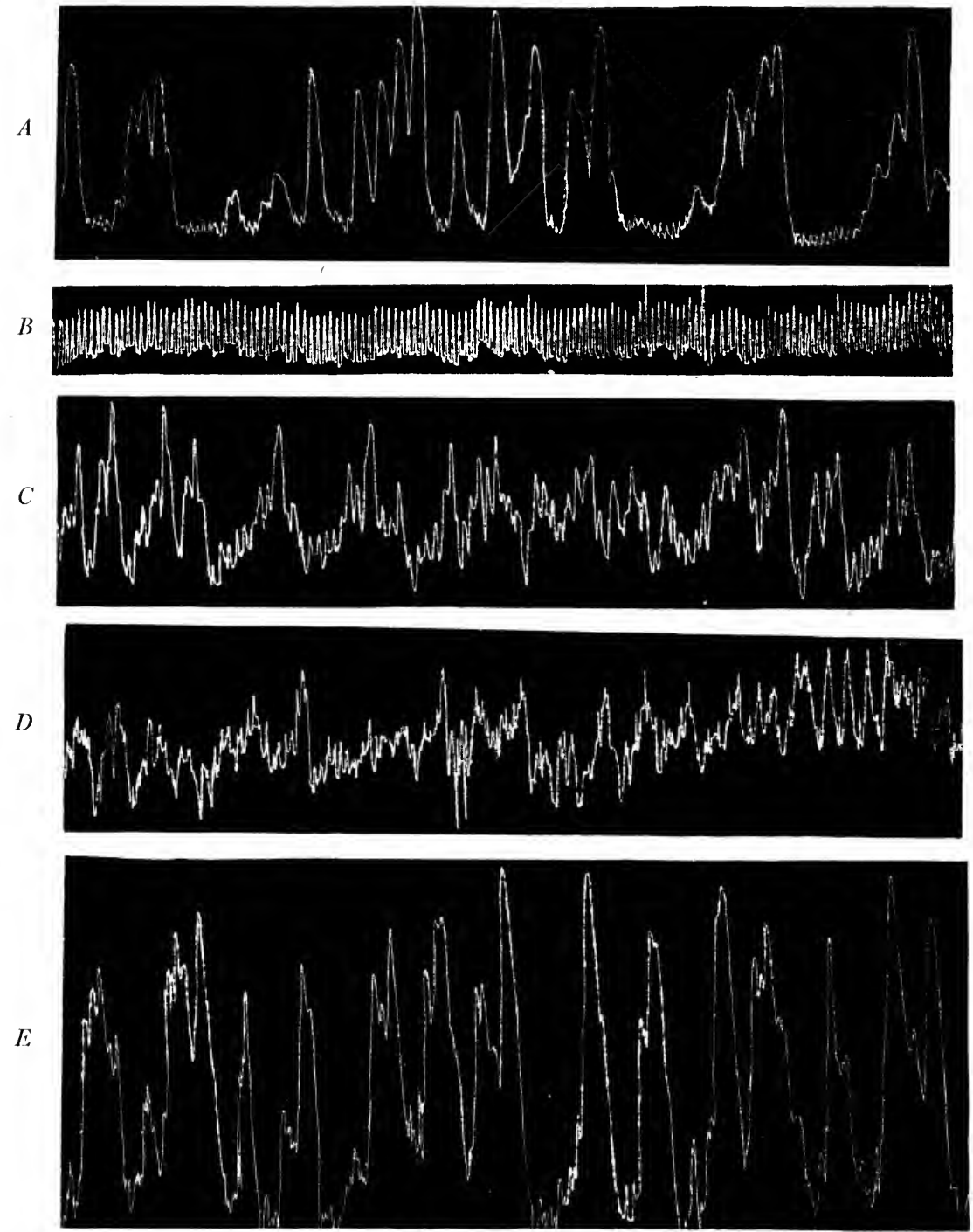

FIc. 32.-Tracings from empty stomach of dog showing effect of alcoholic gastritis on the hunger mechanism (Luckhardt). A, gastric hunger contractions of dog before inducing alcoholic gastritis. $B$, tracing showing atonic stomach of $\operatorname{dog} 24$ hours after excessive administration of alcohol by stomach tube. Dog drinks water and vomits but refuses food. $C$ and $D$, moderate gastric hunger contractions of $\operatorname{dog}$ 3 and 4 days after the alcoholic debauch. Dog ate a little, and vomited frequently, evidently being nauseated and feeling some hunger at the same time. E, vigorous hunger contractions 8 days after inducing the gastritis; dog normal and eating greedily. 
4. Paralysis of the gastric hunger mechanism in pneumonia, distemper, and general peritonitis.-Dogs with well-advanced pneumonia or distemper refuse all food. Such dogs show complete atony and absence of gastric hunger contractions. This is likely the condition of the empty stomach in all acute infections of sufficient severity. The mechanism of the failure of the hunger contractions in these acute infections is not known. The bacterial toxins may depress the motor mechanism of the stomach directly, lower the vagus tonus, augment inhibitory reflexes, or induce excessive secretion of epinephrin. The elevated temperature may also play a rôle. Cannon observed that infections also depress the digestion peristalsis of the stomach and intestines in cats.

5. Depression of the gastric hunger contractions in parathyroid tetany.-Animals in parathyroid tetany show decrease or absence of desire for food in direct proportion to the severity of the tetany and cachexia symptoms. Is this refusal of food due to absence of the gastric hunger contractions?

Our observations were made on three dogs. The dogs were observed every third day for two weeks, so as to secure the average normal gastric tonus and hunger contractions before extirpation of the parathyroid. All three dogs ran a typical course of tetany of varying severity from day to day. Dog I died in tetany on the sixth day after the operation; Dogs II and III died in depression on the eighth and tenth days respectively. The results were practically the same in the three dogs. In this tetany there is depression of the tonus and contraction of the empty stomach parallel with the severity of the tetany, so that during extreme tetany the stomach is practically atonic, and tonus contractions and hunger contractions are completely absent. The milder stages of the tetany (hyperexcitability of the motor nerves, slight tremors, twitchings, and some salivation) may coexist with considerable gastric tonus and hunger contractions, but the hunger contractions are always slower and weaker than normal.

It is well known that the course of parathyroid tetany, especially in dogs, is usually more or less periodic, the animal recovering spontaneously for periods varying from a few hours to a day or 
more between the tetany attacks. Dog II showed two such periods of spontaneous recovery of 34 and 20 hours' duration. During these periods the gastric hunger contractions and the gastric tonus also returned to approximately normal conditions, the dog at the same time taking normal interest in food.

The relation of these depressions of hunger and appetite to the gastric hunger contraction is, nevertheless, not a direct one. During the mild stages of tetany the failure of hunger and appetite is usually much greater than one would expect on the basis of the degree of depression of the hunger contractions. In strong tetany there are no gastric hunger contractions and the dogs refuse food, but the dog may refuse food even though fairly strong hunger contractions are present in mild tetany, or if he does eat the amount of food consumed is very small. It is therefore clear that the depression of hunger and appetite in tetany cannot be accounted for solely on the basis of depression of gastric hunger contractions, although this is unquestionably one of the factors. But we must also take into account either a change in the central nervous system, or a change in the character of the nervous impulses from the stomach and other proprioceptor systems.

The condition of parathyroid tetany, so far as it influences the stomach motor activities, depresses both the digestion movements of the filled and the hunger contractions of the empty stomach, but the movements of digestion show less depression than do the hunger contractions. Thus moderately strong tetany may leave the gastric digestion movements practically normal but completely inhibit the hunger contractions of the empty stomach. The tetany condition does not lead to increased motor activity, either in the empty or the filled stomach.

The cause of this depression of the gastric motor activities in parathyroid tetany is not determined. In the case of the digestion movements it was shown not to be due to splanchnic inhibition. This test has not been made in the case of the hunger movements. But one factor in the depression or complete inhibition of the hunger.contraction in tetany is the increased excitability of the nerveendings in the gastric mucosa. Vomiting is a tetany symptom in 
dogs. And dogs in tetany frequently vomit with nothing in the stomach but bile and saliva. In such dogs water at body temperature introduced into the stomach through a fistula in the fundus causes vomiting. The presence of a delicate rubber balloon in the stomach or the slight inflation of the balloon causes vomiting. This never occurs in normal dogs. The stimulation of the nerveendings in the gastric mucosa in normal animals (man and dog) causes inhibition of the gastric hunger contractions through weak and long reflexes. In parathyroid tetany these nerve-endings in the mucosa become so hypersensitive that they are intensely stimulated by saliva, water, bile, and gastric juice. But in addition to these inhibitory reflexes from the gastric mucosa we probably also have a direct depression of the automatic tissue in the stomach, for it is not likely that the inhibitory reflexes, even though very strong, could maintain the sustained extreme depression seen in strong tetany.

By way of summary, we conclude that parathyroid tetany in dogs does not lead to increased tonus or contractions of the empty stomach, but to depression of the tonus and the hunger contractions. The degree of the depression of the motor activities of the empty stomach is on the whole parallel with the severity of the tetany symptoms, and more marked than the depression of the gastric movements of the digestion. The hyperexcitability of the nerveendings in the gastric mucosa is a factor in this depression. The stimulation of these nerve-endings leads, through local and long reflexes, to inhibition of the tonus and the hunger movements. There is probably also a direct depression of the automatic tissue in the stomach through changes in the blood. The diminution or lack of appetite for food in animals in tetany is on the whole greater than would be expected on the basis of the degree of the depression of the gastric hunger contractions. The cause of the lack of hunger and appetite in tetany is therefore complex. It is due in part to the depression of the gastric hunger contractions. Other factors are the change in the brain, and in the character of the other afferent nervous impulses.

6. Increase in gastric tonus and hunger contractions in dogs after partial occlusion of the pylorus. - It is well known that pyloric 
obstruction in man leads to various disturbances of the gastricdigestion movements, as well as to disturbances of the hunger sensation. In order to determine whether any of the latter disturbances, and particularly the stronger gastric pains that are so frequently in evidence in pyloric obstruction, are due to changes in the motility of the empty stomach, the hunger contractions of the empty stomach after partial occlusion of the pylorus were studied in two dogs by Dr. Elsesser.

The pyloric obstruction was made in the following manner. The serosa extending across the pyloric sphincter about $\frac{3}{4} \mathrm{~cm}$. by $I \frac{1}{2} \mathrm{~cm}$. was scarified, care being taken to avoid injury to the larger blood vessels of that region. Two rows of stitches running parallel to the long axis of the bowel were made, the second folding in the first, thus bringing the two scarified surfaces together and at the same time partially occluding the pyloric lumen. The method has these advantages, that some pyloric obstruction is sure to be produced which will be firmly maintained by adhesions forming between the two raw surfaces. Furthermore, complete occlusion and loss of the animal is avoided, a termination frequently attendant upon placing ligatures around the pylorus. After the animal had recovered sufficiently from the effect of the operation-a matter of several daysrecords of the gastric hunger contractions were made duplicating the normal ones, both as to conditions and periods of time. On one dog, which we may designate as Dog A, a second operation similar to the first was performed and a second series of tracings was obtained.

Dog A remained very well and active and was killed 35 days after the first and I4 days after the second operation. At autopsy the pylorus presented a lumen constricted by a hard, tough mass of granulation tissue, the seat of the scarification and stitching. The stomach was not distended, but showed some hypertrophy. The gastric mucosa lay in deep folds. Dog B became somewhat emaciated after stenosis, evinced a hypersensitivity of the gastric mucosa by occasional vomiting of the balloon while records were being made. At autopsy the pylorus was constricted, so that the stomach contents would be forced through it only with difficulty. The stomach itself was considerably dilated. 
Both dogs gave evidence of delayed emptying of the stomach after the stenosis, in that food remnants were frequently found in the stomach more than 24 hours after feeding.
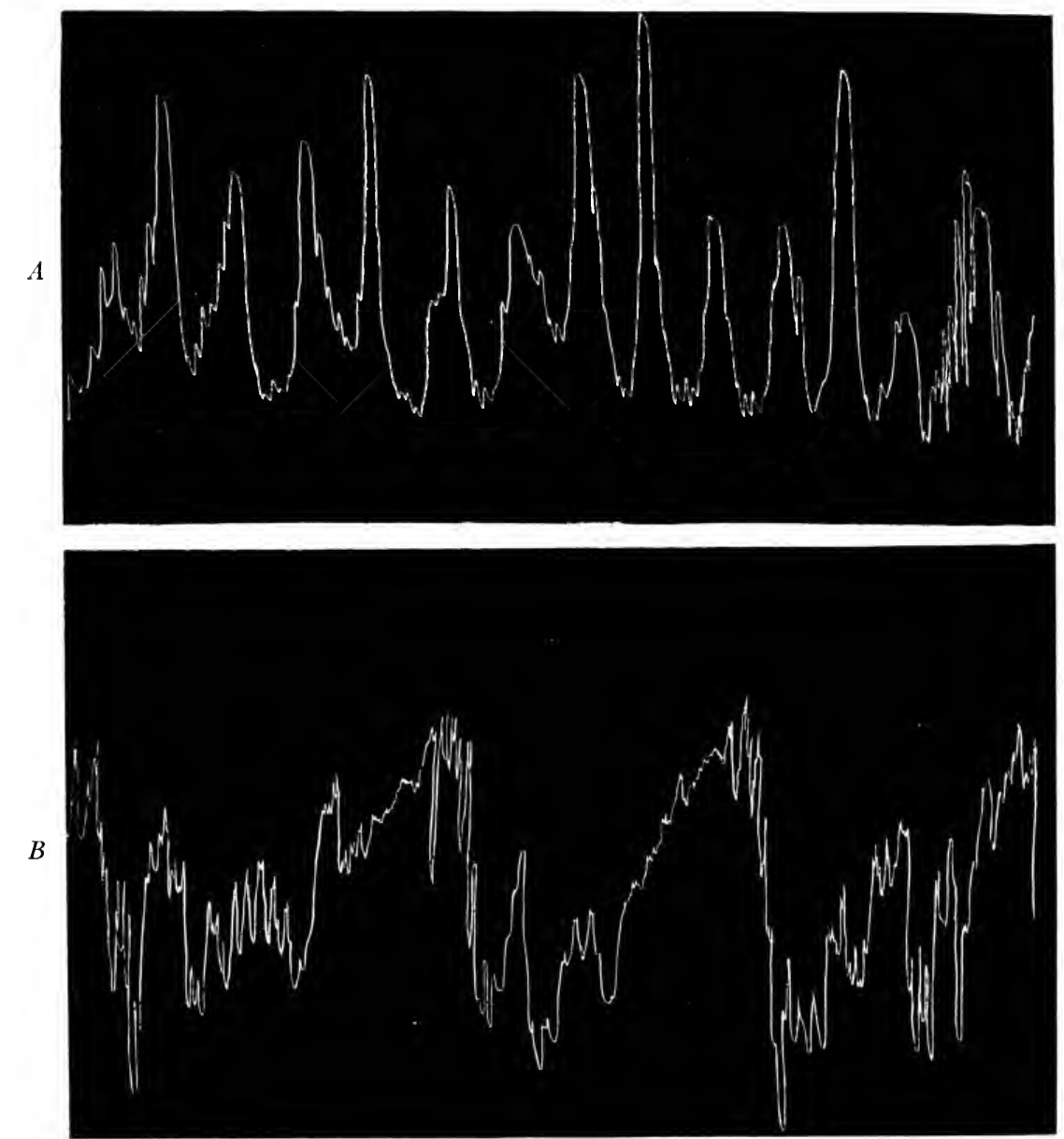

Fig. 33.- Tracings showing contractions of the stomach of dog A. Dog normal; tracing, taken 48 hours after feeding, represents height of a period of hunger contractions; $B$, same $\log$ as in tracing 1,42 hours after feeding, 30 days after production of partial stenosis of the pylorus, showing typical hypertonus and tetany periods of the empty stomach. Chloroform manometer. 'Time, 15 minutes (Elsesser). 
After recovery from the operations the empty stomachs of these two dogs showed more continuous and on the whole stronger hunger contractions than before the operation, with a tendency for the contraction to pass into long periods of incomplete tetanus. It is thus clear that partial stenosis of the pylorus induces a hypermotility in the stomach, irrespective of the presence of food in the stomach cavity. The hypermotility of the stomach during gastric digestion may be a temporary condition induced by the presence of the food and retardation of its passage through the pylorus, a condition similar to that of the small intestine above a region of obstruction. The fact that the hypermotility is present even in the empty stomach seems to show that the motor changes following mere mechanical obstruction of the pylorus are more fundamental and permanent.

The mechanism of this increased motility can as yet only be conjectured. Bacterial toxins from local foci of infection are excluded in these experiments, as the partial stenosis was produced aseptically in animals with normal stomachs and without sub sequent infection. We may be dealing with nervous reflexes from the pylorus involving the entire stomach.

By way of summary, we conclude that partial pyloric stenosis in dogs produces hypertonicity, hypermotility, and hyperperistalsis of the empty stomach, even if of but a few days' or weeks' duration. These motor phenomena are similar to those seen in the filled stomach in man with partial obstruction of the pylorus. The same conditions which lead to hyperperistalsis, etc., during digestion, led at the same time to increased motility of the empty stomach. In other words, partial pyloric stenosis appears to produce a neuromusculár hyperactivity, independent of the presence of food in the stomach.

IV. EXPERIMENTAL INVESTIGATION OF THE HUNGER MECHANISM IN DISEASE OF MAN

I. Absence of gastric hunger contractions in gastritis, tonsillitis, influenza, and "colds."-During the four years that Mr. V., the gastric-fistula case, has been under observation in our laboratory 
he has had a few attacks of mild gastritis, in three cases associated with nose and throat colds, with some temperature. During these attacks the empty stomach remained somewhat atonic with complete absence of the hunger contractions. Mr. V. felt no hunger and had little or no desire to eat. In fact, putting food in the stomach in these conditions sometimes produced nausea. The absence of hunger and depression of the appetite thus ran parallel with the impairment of the gastric hunger mechanism.

Luckhardt and Hamburger have reported gastric atony and absence of hunger contractions in a case of acute gastritis brought on by dietary indiscretion. The author has taken records on himself in one mild attack of gastritis, lasting three days, two attacks of "cold" and tonsillitis, one of which was complicated with painful antrum infection. During the gastritis the empty stomach showed no hunger contraction. The tonsillitis, "colds," and antrum infection did not completely abolish the hunger contractions, except when sufficiently severe to induce elevation of the body temperature to $101^{\circ}$ or $102^{\circ} \mathrm{F}$. Again, the depression or absence of the feeling of hunger ran parallel with the degree of depression of the gastric hunger contractions. But even when no hunger was experienced, the sight of palatable food was capable of inducing some appetite.

2. Hunger in diabetes mellitus.-Dr. Luckhardt studied the gastric hunger contractions of a man twenty-nine years of age, in the last stage of diabetes. The empty stomach showed abnormally strong hunger contractions till within a few days of death in coma, the patient at the same time complaining of great hunger. This seems to show that in clinical diabetes there is an increased activity of the gastric hunger mechanism similar to that demonstrated in pancreatic diabetes in dogs. But more observations are needed on clinical diabetes before this relation can be accepted or demonstrated.

3. Gastric hunger contractions in a case of gastric cancer.-The subject was an old man, considerably emaciated, but with no serious obstruction at the pylorus. His hunger and appetite were good and his gastric hunger contractions normal. This observation 

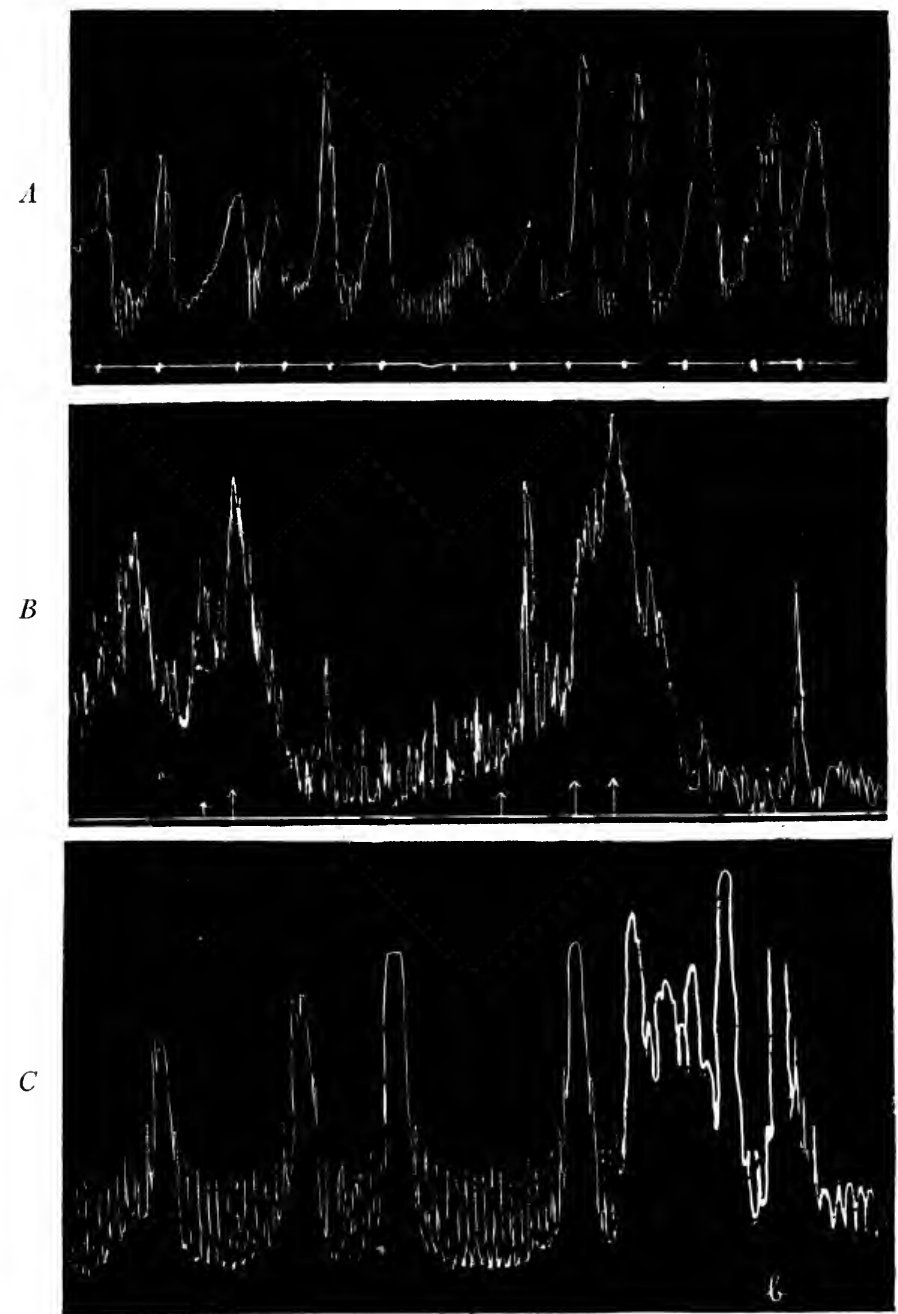

FIG. 34.- $A$, normal hunger contractions of the empty stomach of a neurasthenic man. The contractions gave rise to epigastric pain, not hunger pangs. $B$, tetanic contractions of the empty stomach of a man with carcinoma of the stomach and partial pyloric obstruction. The contractions caused epigastric distress, rather than true hunger. $C$, vigorous hunger contractions of the empty stomach of a diabetic man, 5 days before death in diabetic coma, showing vigorous hunger contractions associated with the diabetic polyphagia (Luckhardt and Hamburger). 
of Luckhardt and Hamburger is in line with the fact that patients with cancer of the stomach may continue to feel hunger when the tumor growth is so advanced that feeding by mouth becomes impossible.

4. Excessive pain produced by normal gastric hunger contractions in neurasthenics.-Dr. Luckhardt reports a case of a man who sought hospital treatment for a dull ache or epigastric pain which came on whenever the stomach was empty. This pain or ache proved to be due to the hunger contractions of the empty stomach, the latter not being stronger than those observed in normal individuals. The patient believed that the observation of his stomach by the balloon method was a method of treatment. The patient stated after a few days that the pain had ceased entirely, although the gastric hunger contractions continued normal, and he left the hospital very grateful for what had been done for him. There was no evidence of organic lesion in the stomach. This case presents either a temporary neurosis (hyperexcitability) of the gastric hunger nerves so that the normal contractions actually give rise to abnormally strong impulses, or else the normal impulses from the stomach become exaggerated in consciousness through perverted attention.

The author studied the gastric hunger contractions of a similar case, a young farmer seeking medical aid for excessive epigastric pain, and some feeling of weakness and depression. No organic lesions could be detected. The gastric hunger contractions were strong, the periods ending in incomplete tetanus, but equally strong hunger tonus and contractions have been seen in normal individuals of his age and occupation. Yet the tonus and contractions were to him sufficiently painful to seek medical aid repeatedly, but no treatment appeared to give permanent relief.

5. Cause of the "hunger pains" in cases of gastric and duodenal ulcers.-Hamburger, Ginsburg, and Tumpowsky found that the "gnawing" hunger pains in duodenal and gastric ulcers are caused by the hunger contractions of the empty or partly empty stomach. These contractions are, on the whole, not stronger than those of healthy persons in hunger, yet they are felt as much more painful 


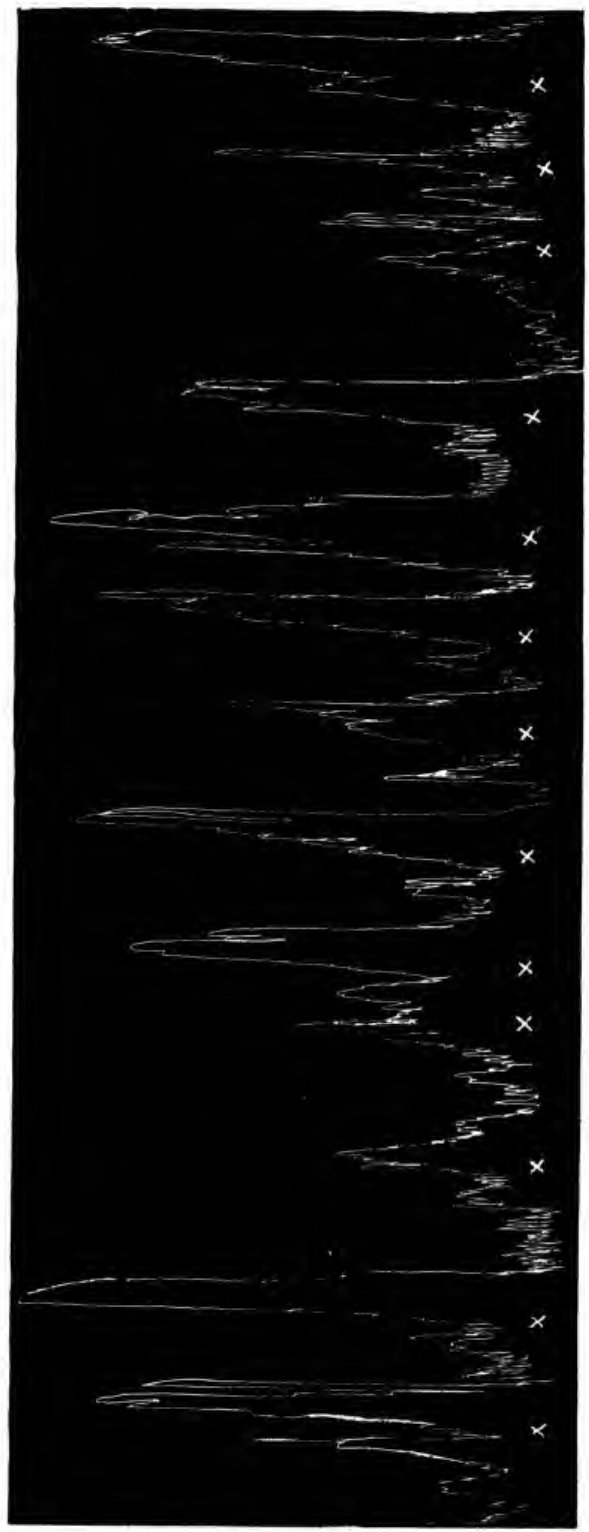
实密 50 s0 过总苛 吾昰 . 돌 要 so of 我 $=$ 㤩芯 동 열 멸 을 졍

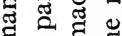
\&

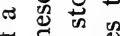
$\overrightarrow{0} \tilde{z}$ 융 药 卷

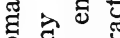
के 它落

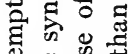
( $\Xi \nsubseteq .5$ ㄴㅇㅇ : 늘 = 矛 प्र

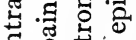

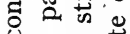
岁

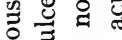
㟧 峁 .00 ○े है ఫ ड્ bo o की 鸪 की $\infty \mathrm{D}$. 든 要 话 它苋范 的岕导岕昰 نं

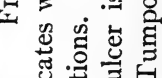


than the normal hunger pangs. This may be due to hyperexcitability of the sensory nerve-fibers in the stomach. These patients usually feel a more or less steady and dull gastric pain besides the gnawing or intermittent pains associated with the rhythmical contractions. The cause of the continuous but less sharp pain is probably to be sought in the steady and strong tonus contractions of some region of the stomach or duodenum. This steady but dull gastric pain in ulcer patients is thus similar in origin to that felt by some people in prolonged starvation.

If the theory of infectious origin of ulcers indicated by the work of Rosenow is applicable in all cases, the hyperexcitability of the gastric sensory nerves in ulcer is probably due to the local inflammatory processes. Even in ulcers of traumatic origin (chemical, mechanical) secondary infection probably takes place, so that even in such cases we have local inflammation.

6. Cause of gastric pain in diseases of the gall bladder.-We do not refer to the pain definitely due to the mechanical stimulation of the gall bladder or the bile duct by biliary calculi, but to the epigastric pains that come on a certain length of time after a meal, and closely resemble the hunger pains of ulcer. We know that the rhythmical contractions of the gall bladder are greatly augmented during gastric digestion, probably through reflex stimulation of acid chyme in the duodenum. In this manner, pain from the gall bladder itself may be augmented parallel with gastric digestion peristalsis especially in cases of hypersecretion.

Ginsburg and Tumpowsky studied the epigastric pain in one patient with gall-bladder infection, as determined by clinical diagnosis. Gastric and duodenal ulcers were excluded. In this patient the pain ran absolutely parallel with the strong hunger contractions of the empty or nearly empty stomach. The hunger contractions were stronger than usual for a man of the patient's age. We cannot be sure that the gastric contractions were the only cause of the pains; there may be strong contractions of the gall bladder parallel with the stomach contractions.

In this patient, too, the epigastric pain appeared greater than warranted by the strength of the gastric contractions. Evidently 
gall-bladder infections may lead to hyperexcitability of the sensory nerve-fibers of the stomach and duodenum, possibly from the spread of bacterial toxins along the branches of the vagi nerves.

7. Hunger contractions of the empty stomach in infants with pylorospasm and congenital pyloric stenosis.-Pylorospasm has been ascribed to a great variety of causes, including primary neurosis of the local motor mechanism. The hyperperistalsis of the filled stomach usually associated with spasms of the pylorus may be a temporary condition due to the presence of food in the stomach. On the other hand, if pylorospasm is simply an expression of primary hypermotility of the entire stomach, this condition of hypertonus and hypermotility should also be in evidence when the stomach is empty. A study of the motor conditions of the empty stomach may thus aid in determining some of the factors involved in hypercontractility of the pyloric sphincter. In infants "rumination" is probably always secondary to chronic vomiting, which in turn may or may not be associated with pylorospasm. If the vomiting is due to gastric hypertonicity and hypermotility, these conditions should also be present in the empty stomach, with or without the involvement of the pylorus.

We have studied the motor conditions of the empty stomach in two infants, one with congenital pyloric stenosis, and one with pylorospasm, chronic vomiting, and rumination.

Case I: Infant three months old; chronic vomiting and gradual loss of weight. Congenital pyloric stenosis. Gastro-enterostomy was made. The pylorus was found contracted, and somewhat edematous and anemic. Before the operation, record of the tonus and contractions of the empty stomach was made by the balloon method as applied to infants. The strength of the contractions was markedly greater than in normal infants. The duration of the periods of contraction was also greater. This indicates a greater than normal gastric tonus. There was no indication of prolonged tetanic contractions.

Case 2: Infant five months old; chronic vomiting ("rumination"). Practically stationary body weight. Pylorospasm. A number of observations were made on this infant. When the child 

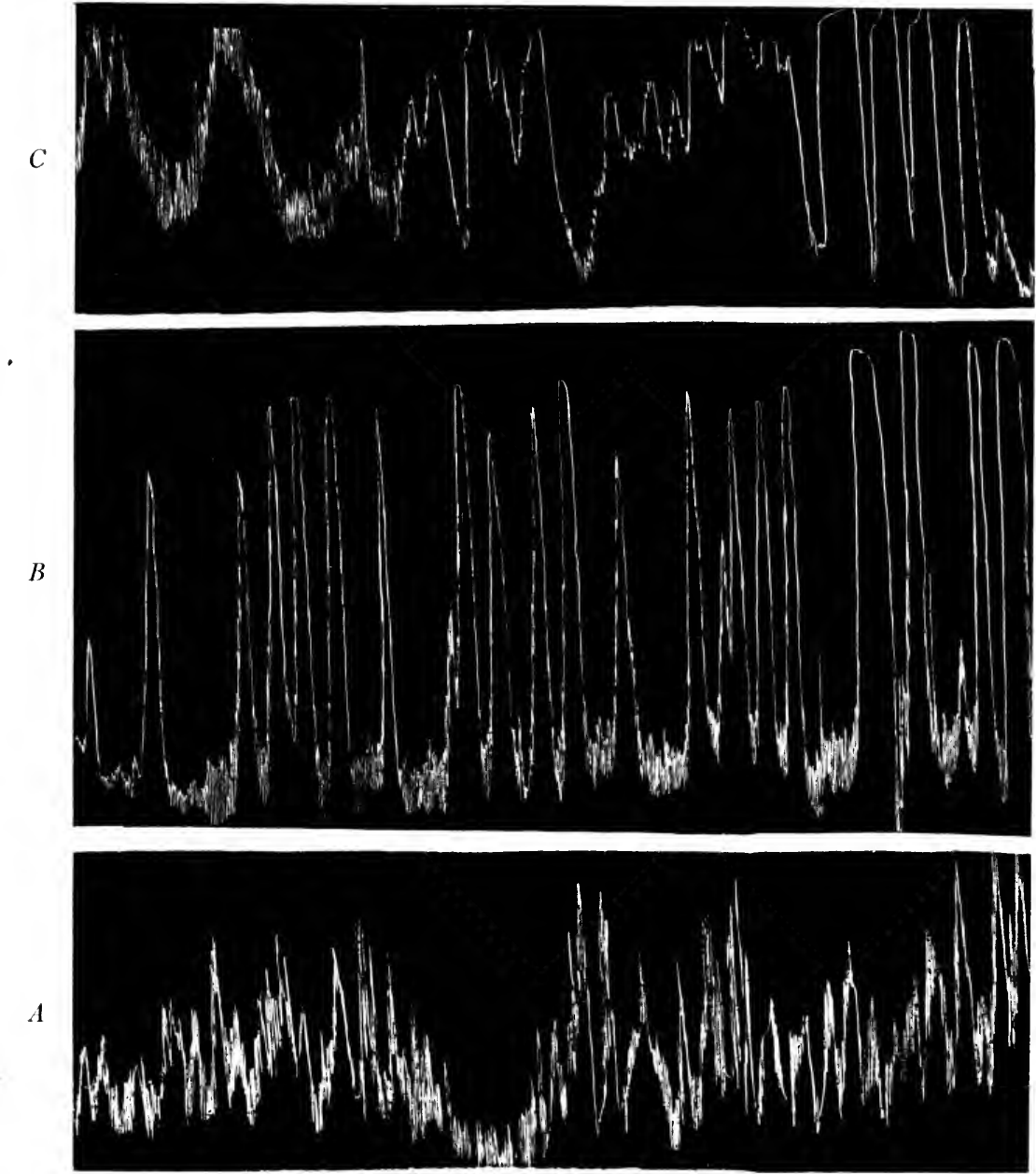

FIG. 36.-A, tracing showing a period of vigorous hunger contractions of the empty stomach of a normal infant; $B$, tracing showing exceptionally intense and practically continuous hunger contractions of a 3 -months-old infant with persistent pylorus spasm amounting to almost complete pyloric obstruction accompanied by chronic vomiting and gradual loss of weight; $C$, tracing showing exceptionally intense hunger contractions and periods of incomplete tetanus of the empty stomach of a 5 -months-old infant with chronic vomiting ("rumination") and practically stationary body weight. In the right half of the tracing the upward excursion of the manometer had to be checked mechanically to prevent the chloroform from being driven out. Hence the extreme vigor of the gastric contractions is not fully registered. Chloroform manometer. Time 20 minutes. 
was quiet, so that all nervous inhibitory factors were eliminated, the empty stomach usually showed hypertonus with periods of tetanic contractions lasting several minutes, interspersed with vigorous contractions of normal duration-an unmistakable condition of hypertonicity and hypermotility. If the infant was asleep during the observation period the tetanic contraction of the stomach invariably caused restless facial grimaces, or he would wake up and cry. Such vigorous and prolonged periods of tetanic contractions have so far never been observed in the empty stomach of normal infants. They have been observed in adult persons and in dogs after prolonged starvation. This type of contractions of the empty stomach may also be seen in dogs with pancreatic diabetes.

The results in the cases of these two infants indicate that pylorospasm and pyloric stenosis involve either primarily or secondarily a condition of hypertonus and hypermotility of the entire stomach. The excessive contraction of the pylorus may be an expression of this general hypermotility. It is known that the tonus and contractions of stomachs in young mammals are greater than in the adult and in the old. This may be correlated with the greater tendency to hyperactivity of the pylorus in infancy and childhood. In the adult these gastric contractions would cause intense hunger pains, and it is probable that such pains are also experienced by the infant.

8. Hunger in experimental fevers in man and dogs.-Dr. Rupp induced temporary fever in himself and in a number of other men by injections of typhoid vaccines and of sodium nucleate. Records of the gastric hunger contractions were taken in the usual way, and careful notes made of the subject's own feeling of hunger and appetite. Fever reactions on injection of the standard doses of typhoid vaccine are usually very slight and variable, but with doses two or three times larger elevation of the temperature up to $103^{\circ}$ or $104^{\circ} \mathrm{F}$. together with the other symptoms of fever can usually be produced for a few hours. Sodium nucleate invariably brings on a temporary fever, the degree and duration of the fever depending on the quantity injected. 

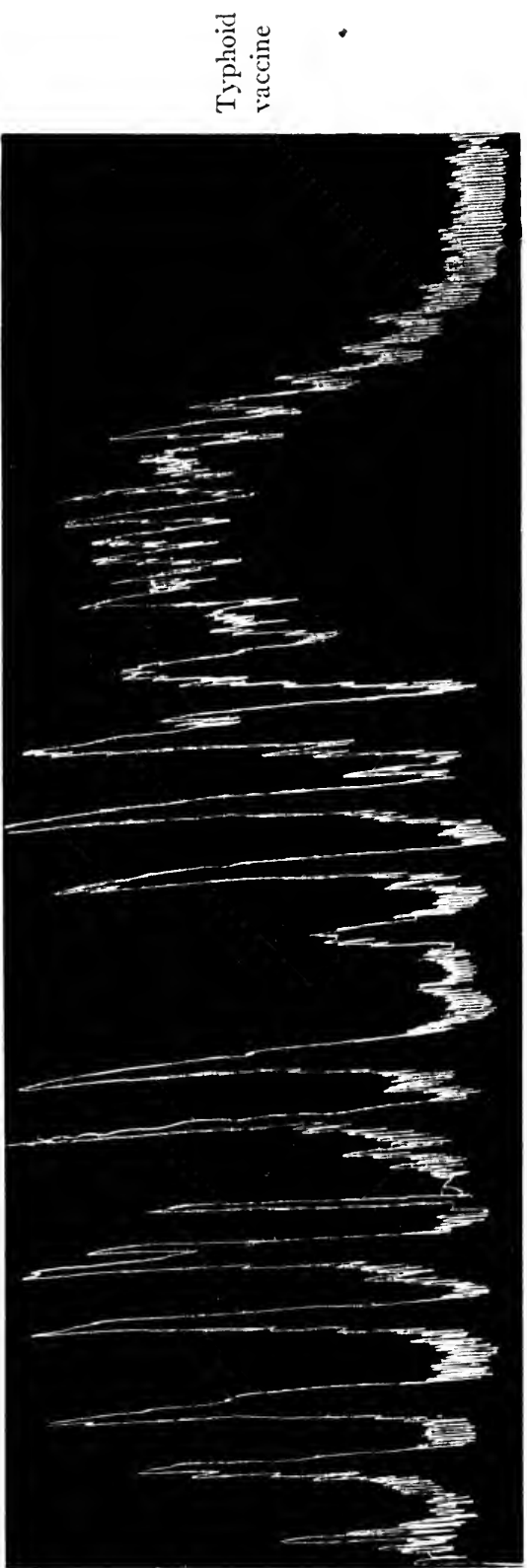
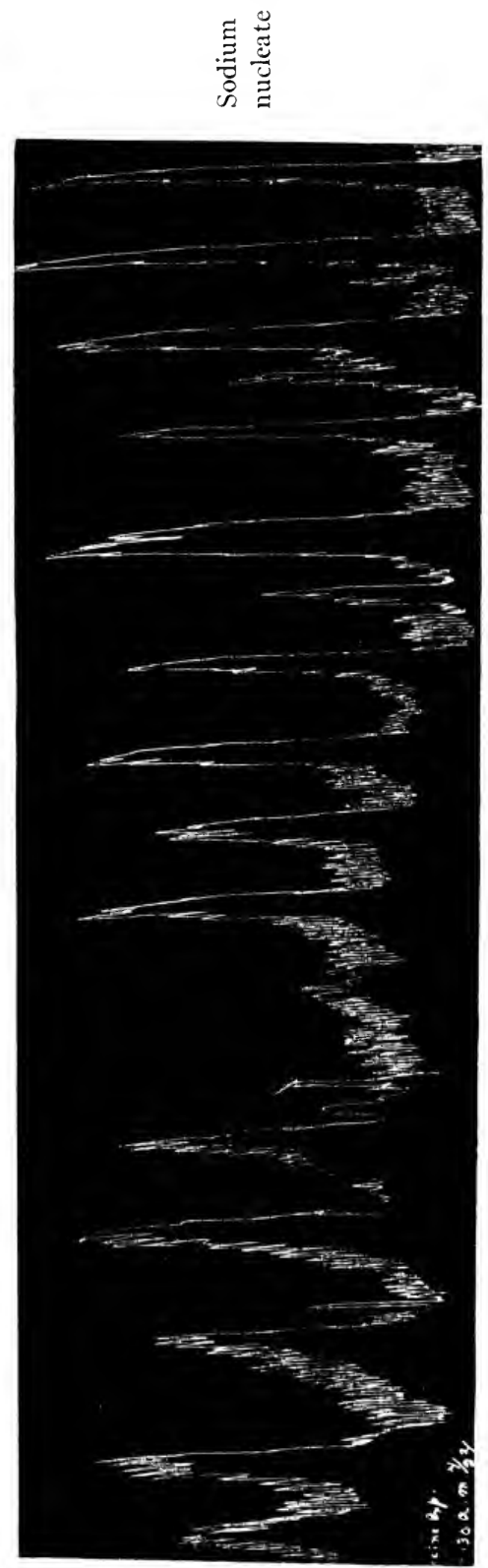

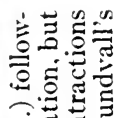

的荡

in

ن

$\approx$ 边 $\ln ^{2}$.

胥承.

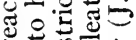

㻤

- 5

巳

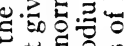

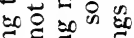

.

궁흐

Е

跑.

는.을

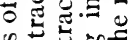

क ज证

용요

过

Е

क्ष

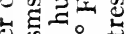

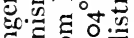

등 유으

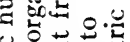

을

的可可

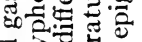

ज证。

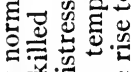

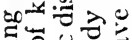

등.

记

舫.

$\infty 0$.

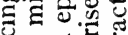

손

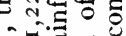

的记

1 둥워

$\therefore$ 为曹

m. 글

ن. 尫灾

工. 串造豆 
Dr. Rupp found that when the temperature elevation reached only $100^{\circ}-102^{\circ} \mathrm{F}$. the strength and duration of the gastric hunger contractions showed practically no deviation from the normal. But they produced a different effect on consciousness. Instead of the normal hunger pangs associated with increased appetite and desire for food, the gastric hunger periods during the fever produced headache, nausea, and an epigastric distress like "sick stomach." More severe temperature reactions ( $104^{\circ}-\mathrm{rO} 5^{\circ} \mathrm{F}$.) usually render the empty stomach atonic, and in that condition there are no hunger contractions. When the subjects experienced the fever chills there was always complete absence of the gastric hunger contractions.

Similar results were obtained by Dr. Mayer on dogs. Sodium nucleate fevers of $103^{\circ}-105^{\circ} \mathrm{F}$. lead to gastric atony and absence of the hunger contractions. But temperature elevations of one or two degrees above the normal had little or no effect.

It must be noted that we are here dealing with very temporary fever reactions. It is not unlikely that temperature elevations of one or two degrees above the normal and persisting for days or weeks would have a more deleterious influence on the gastric hunger mechanism.

These experiments show that fever anorexia is in some cases at least more complicated than the mere absence of the gastric hunger contractions. In fact we may have objectively normal gastric contractions parallel with the epigastric feeling of "sick stomach," nausea, headache, depression, no thought of or desire for food. This anomalous condition may be due to hyperexcitability of the gastric sensory nerves by action of the toxins, or due to elevation of the temperature. Changes in the central nervous system may also play a rôle. 


\section{CHAPTER XVII}

\section{HUNGER AND APPETITE IN DISEASE-(Continued)}

\section{ACTION OF BITTER TONICS}

ACTION ON THE HUNGER MECHANISM

The value of "bitter herbs" in disease is a current popular belief, and the use of these bitters as medicine probably antedates even the "medicine man" of primitive human society. Today the use of bitters is, or at least should be, confined to cases of impaired gastric digestion. The mechanism of this action is still in the main an open question, despite a considerable amount of experimental work. The literature seems to show that the bitters cause increased secretion of gastric juice. Nevertheless, Cushny concludes that the favorable effects from bitters are largely subjective. The bitters are capable of producing a considerable impression on the patient, so that their effects may be due in part to suggestion and not to any real action of the drug. That the bitters increase appetite and hunger is a generally accepted view. The bitters may produce these results in any or all of the following ways: (I) direct inauguration or augmentation of the hunger contractions of the empty stomach, or hastening the reappearance of the gastric hunger contractions by facilitating gastric digestion (through increased secretion or increased peristalsis), and thus accelerating the emptying of the stomach; (2) augmentation of appetite directly by stimulation of gustatory and other sensory nerves in the mouth, esophagus, and stomach, or indirectly, by accelerating gastric digestion; (3) central Bahnung or facilitation, the strong afferent impulses from the mouth (and possibly also from the esophagus and stomach) may in some way bring feeble hunger and appetite sensations more prominently into consciousness.

The tests were carried out on a number of normal men, and the influence of the bitters in the mouth and in the stomach was tested separately. The bitters were introduced through the stomach tube 
when it was desired to study the action from the stomach alone. In Mr. V., and usually also in the dogs, the drugs were introduced through the gastric fistula.

In order to exclude possible psychic factors, the subject was frequently kept in ignorance of the nature of the substance (water or drug) as well as of the time when it was given.

The following bitters were used: tinctures of gentian, quassia, columba, humulus, and condurango; elixir of quinine, strychnine, and iron.

In the case of man the bitters were introduced into the stomach in varying quantities up to the maximum therapeutic dose, but never exceeding that. In the case of the dogs the drugs were used in gradually increasing quantities until definite effects were produced.

I. Action in the stomach.-When used in therapeutic quantities and introduced directly into the stomach so as not to come into contact with the mouth or esophagus, these bitters have no direct action whatever on the hunger mechanism. If the bitters are introduced into the stomach during a hunger period, the gastric hunger contractions continue in their normal rate and strength until the completion of the hunger period, except for a slight temporary inhibition that may appear immediately on introduction of the drug. This transient inhibition is not peculiar to these drugs, however, since it follows the introduction of any liquid, including water at body temperature, directly into the stomach. At no time did we obtain an increase in the gastric hunger contractions from the bitters. If the bitters are introduced into the empty but quiescent stomach, that is, between two hunger periods, there is no immediate initiation of gastric hunger contractions or hastening of the appearance of the next hunger period.

The foregoing results were obtained with all the bitters both in man and dogs, so that we are inclined to believe that all the numerous bitters used in therapeutics are without direct action (that is from the.stomach) on the hunger mechanism when used in therapeutic quantities.

When the bitters are introduced directly into the stomach in sufficient quantities to produce demonstrable effects on the hunger 
mechanism, this action is always in the direction of inhibition, and the inhibition is the greater the greater the quantity of the drug. All the bitters tried are capable of causing this inhibition of hunger. It is not known what ingredient in the bitters is responsible for this action. The alcohol in the tinctures will itself produce some inhibition. This inhibition is not peculiar to the bitters, as previous work has shown that anything which stimulates the nerve-endings in the gastric mucosa inhibits the gastric hunger mechanism in proportion to the intensity and duration of the stimulation. And it is of little or no interest in therapeutics, as the quantities of the drugs required to produce it are much greater than that permissible in practical medicine. When the elixir of iron, quinine, and strychnine is put into the stomach in quantities sufficient to affect the hunger mechanism, the animal usually develops mild symptoms of strychnine poisoning (rapid respiration, greatly increased reflex excitability). During this period of increased activity of the skeletal neuromuscular mechanisms, there is
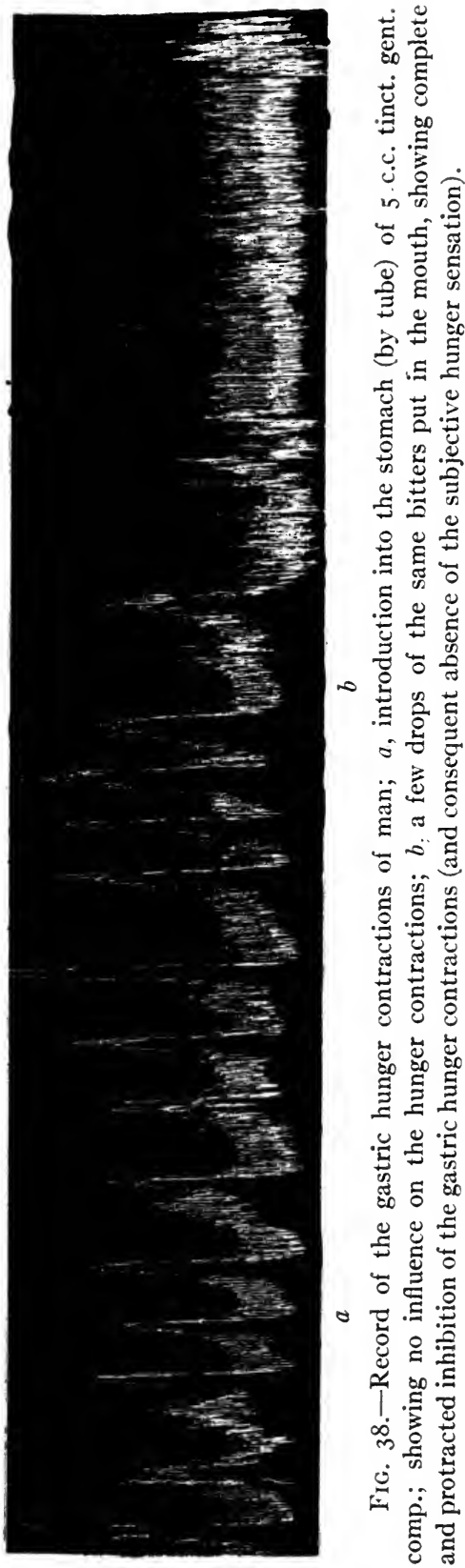
not only no increase in tonus and hunger contractions of the empty stomach, but the stomach is rendered even more atonic than during the quiescent interval between the hunger periods in the normal animal, a situation similar to that found in animals in parathyroid tetany. According to Heubner and Reider, large doses of bitters retard the emptying of the stomach in digestion.

2. Action in the mouth.-The experiments on dogs were unsatisfactory and inconclusive, for the reason that the dogs object to the bitters, even when given in minute quantities by the mouth. The dogs salivate profusely, become restless, and endeavor in various ways to get rid of the apparently disagreeable taste of the bitters. Putting small quantities of the bitters in the mouth invariably leads to prompt and prolonged inhibition of the gastric hunger contractions, but inhibition of the hunger pangs in dogs accompanies restlessness of the animal from any cause. The results on man are not complicated by the uncontrollable factor of struggling and protest.

In man the bitters acting in the mouth inhibit the gastric hunger contractions in direct proportion to the intensity of their stimulation of taste sense. If the bitters are placed in the mouth during a period of quiescence of the empty stomach, there is no initiation of gastric hunger contractions, but so far as any effect can be detected this is a temporary inhibition of the gastric tonus. The temporary inhibition of the gastric tonus.and hunger contractions by the bitters acting in the mouth is not followed by an increase in gastric tonus or gastric hunger contractions above the normal. That is, there is no after-effect of the nature of augmentation or stimulation of the hunger mechanism. A few drops of tincture of condurango on the tongue may inhibit the hunger contractions for $I_{5}$ to 20 minutes.

We think that a sufficient number of bitters is included in the experiments above to justify applying the results to the whole group of bitters, especially in view of the fact that the action on the hunger mechanism is the same in all cases.

These experiments were conducted on normal individuals, while the bitters are or should be prescribed only in cases of anorexia, 
a disordered digestion, or particularly a disordered gastric digestion. There remains the possibility of a more favorable action of the bitters on the hunger mechanism in these pathological conditions. It is desirable that direct tests be made on such material with proper control. But we venture to predict that the results will be the same as those reported above on normal men, as it is not likely that the fundamental reflex relations of the sensory nerves in the mouth and the gastric mucosa to the hunger mechanism are so readily changed by disease.

It must be noted that the reflex inhibition of the hunger contractions from the actions of the bitters in the mouth is accompanied by cessation of the conscious hunger pangs. There is a complete parallel between the objective inhibition and the subjective absence of hunger sensation.

The foregoing results show clearly that the bitters usually employed in therapeutics have no favorable action on the hunger mechanism. But the therapeutic use of these substances may still be justified by their direct action on gastric secretion or on the appetite.

Summarizing, we conclude that in therapeutic quantities the bitters, acting in the stomach alone, have no effect on the gastric tonus or the gastric hunger contractions or on the parallel sensation of hunger. In greater than therapeutic doses the bitters inhibit the hunger contractions and abolish the hunger sensations, probably by stimulation of nerve-endings in the gastric mucosa. Acting in the mouth alone, the bitters, even in traces, inhibit the hunger contractions and abolish the hunger sensations in direct proportion to the intensity and duration of the stimulation in the mouth, and there is no after-effect in the way of augmentation of hunger contractions and hunger sensations.

\section{ACTION ON THE SECRETION OF GASTRIC JUICE IN NORMAL MEN AND DOGS}

The literature on this subject is both considerable and conflicting. It seems pretty well established that the bitters have no action on the pepsin-hydrochloric-acid digestion itself, except possibly in 
way of slight retardation. This slight retarding action is of no practical significance, especially when the bitters are taken 20 or 30 minutes before the meal, or even just before the meal, because of the great dilution with gastric juice, saliva, and the fluids of the food. The literature also points to the conclusion that by themselves the bitter tonics are incapable of causing secretion of gastric juice, either by acting in the mouth or in the stomach. Pavlov noted in dogs that the bitters acting in the mouth cause a copious flow of saliva, but leave the gastric gland perfectly quiescent; and not even when introduced into the stomach do they cause secretion of gastric juice.

Some observers (Reichmann, Scheffer, and others) have reported that as long as the bitters remain in the stomach they depress or diminish the secretion of gastric juice, and this led to giving the bitters from ro to 30 minutes before the meals.

Do the bitter tonics augment the secretion of gastric juice indirectly by increasing the excitability of the nerve-endings of taste in the mouth, and possibly the nerves of appetite sense in the stomach? This is the view emphasized by Pavlov, but he does not adduce any experiments in its support. The work of Borissow seems to confirm it, however. Borissow reports I 2 sham-feeding tests on one dog with gastric fistula and esophagotomy. Six of these tests were made after giving the dog tincture of gentian in the mouth. In both series of tests the sham feeding was continued for I minute and the gastric juice collected for 2 hours following the sham meal. In the case of the gentian series, the sham feeding was instituted as soon as the profuse salivation induced by the bitters had ceased. Borissow obtained the following results:

\begin{tabular}{|c|c|c|c|}
\hline Series & Min. c.c. & Max. c.c. & Aver. c.c. \\
\hline $\begin{array}{l}\text { Normal or no tonic. . . . . . } \\
\text { Tincture of gentian. . . }\end{array}$ & $\begin{array}{l}59 \cdot 3 \\
85 \cdot 1\end{array}$ & $\begin{array}{l}126.5 \\
185 \cdot 2\end{array}$ & $\begin{array}{l}101 \\
130\end{array}$ \\
\hline
\end{tabular}

There was no difference in the acidity and the pepsin concentration of the gastric juice of the two series, but the average excess 
of gastric juice in the gentian series is striking. However, it may be questioned whether a short series of tests on one animal can be held as conclusive, especially in view of the great individual variations (over Ioo per cent) within each series.

Experimental procedure on man.- It will be recalled that Mr. V. has the esophagus completely restricted at the level of the upper end of the sternum, so that nothing can be swallowed from the mouth and reach the stomach via the esophagus. Above the constriction the esophagus is somewhat dilated so as to hold about half a glass of liquid. These conditions serve admirably for studying the influence of the bitters on the secretion of gastric juice.

On all test days roo c.c. of water were put into the stomach I 20 and 60 minutes before the meal, so as to insure a completely empty stomach. The tonics were introduced into the stomach via the fistula $I_{5}$ to 30 minutes before mealtime. In the series of tests with the tonics in the mouth, these were put into the mouth and swallowed into the esophageal pouch ro minutes before the meal. They usually had to be expectorated before the meal actually began, because of the salivation induced by them.

In all three series of tests the gastric juice was collected in the course of the first 20 minutes during which Mr. V. was chewing his food in the usual way, preparatory to putting it into the stomach by means of a syringe. We are therefore dealing with the appetite gastric secretion only.

The stomach was invariably emptied just before Mr. V. started to eat, and a record was kept of the quantity, acidity, and pepsin strength of this juice found in the empty stomach, as it was thought that the quantity and quality of this juice might serve to indicate the physiologic condition of the gastric glands, irrespective of the condition of the appetite.

The experiments were made during the period from April to November, I9I4. The tests with the tonics were interspersed with controls without the tonics all the way, so as to eliminate as far as possible the errors from variations in nutrition, appetite, etc., associated with variations in climate and bodily activity. 
It was aimed to make these tests a mere incident in Mr. V.'s daily routine. For that reason no special dietary standard was fixed. The ingredients of the noonday meal Mr. V. selected for himself at a nearby cafeteria. He naturally selected what appeared to him most palatable from day to day. The evening meal was taken in the same boarding-house throughout the experimental period. Mr. V. stated that the boarding-house meals were very much the same from week to week, and were less palatable than the noonday meal.

The tonics used (in therapeutic doses) were tinctures of gentian, quassia, columba, humulus, and condurango, and elixir of quinine, strychnine, and iron. Most of the tests were made with the gentian tincture and with the elixir.

In order to exclude all possible psychic factors, Mr. V. was not told of the purpose of the experiments. He went about his daily work, taking his usual food at the usual time, while now and then a tonic was given and the appetite secretion measured. We think it may safely be concluded that such psychic factors as faith in the potency of or hope of improvement from the drugs was entirely eliminated. Nor was the taking of any of these tonics by the mouth disagreeable or loathsome, such as might induce psychic depression.

\section{TABLE V}

Gastric Juice Secreted by Mr. V. during the First Twenty Minutes of Chewing Food

\begin{tabular}{|c|c|c|c|c|c|}
\hline \multirow{2}{*}{ MeaL } & \multirow{2}{*}{ Tonics } & \multirow{2}{*}{$\begin{array}{l}\text { No. of Ex- } \\
\text { PERIMENTS }\end{array}$} & \multicolumn{3}{|c|}{ Gastric Juice in c.c. } \\
\hline & & & Lowest & Highest & Average \\
\hline \multirow{3}{*}{ I $2: 00-I$ :O० P.M. } & No tonics. . . . . . & $3 \mathrm{I}$ & 35 & 95 & 58.0 \\
\hline & Tonics in mouth... & 30 & 30 & 93 & 61.0 \\
\hline & Tonics in stomach. & 20 & 40 & 85 & 58.2 \\
\hline \multirow{3}{*}{ 6:00-7:०० P.M. } & No tonics........ & 20 & 20 & 50 & 36.0 \\
\hline & \{Tonics in mouth... & 20 & 20 & 48 & 33.0 \\
\hline & (Tonics in stomach. & I5 & I8 & 47 & 30.1 \\
\hline
\end{tabular}

Tests to the number of 50 were made with bitters in the mouth, and 35 with bitters in the stomach, together with $5^{\mathrm{I}}$ control tests. These are summarized in tables. The lunch and supper series are 
tabulated separately, because the appetite gastric secretion was uniformly less at the evening meal. This is probably due to less palatable food at the evening meal (absence of variety in the food, inferior cooking).

Examination of the tables shows that the bitter tonics acting either in the stomach or in the mouth are without influence on the quantity of the appetite psychic secretion. There is a suggestion of an increased secretion with the bitters in the mouth for the noonday series, but this is counterbalanced by a slightly lower figure for the evening meal. The average physiologic condition of the gastric glands is practically the same in all the series.

As Mr. V. is a young man in good health, the question naturally arises whether the sensory nerves for the appetite sense (in mouth and stomach) are not normally in a state of maximum excitability, so that no further increase in excitability by bitter tonics is possible. This objection seems to be met by the lower secretion at the evening meal. Here is a condition in which less palatable food should have been rendered more palatable by contrast with the bitter tonic, or by actual increase in the excitability of the gustatory nerves. The results indicate no improvement by contrast. If the bitters in the mouth augmented the excitability of the taste nerves at the evening meal, this evidently caused an already unpalatable food to taste still more unpalatable, and hence the diminished appetite secretion.

These bitters in mouth or stomach produced no change in the acidity and in the pepsin concentration of the appetite gastric juice.

A similar series of tests was made on eight dogs provided with an accessory stomach pouch, according to the Heidenhain-Pavlov method. Care was taken to interfere as little as possible with the distribution of the vagi to the stomach pouch.

The tonics were introduced into the main stomach by means of a stomach tube, so as not to come in contact with the nerveendings in the mouth or esophagus. They were introduced into the stomach pouch directly with a pipette. When introduced into the mouth the bitters were soaked up in a small wad of cotton and the dog was compelled to chew on this for I minute. The tests were made 24 hours after the previous meal, and each dog was fed a 
fixed quantity of boiled lean meat. The gastric juice was collected for 2 hours after the beginning of the feeding.

The tonics were put into the main and accessory stomachs from 20 to 30 minutes before feeding, and into the mouth ro minutes before feeding. The size of the dogs varied from 5 to $8 \mathrm{~kg}$. The quantity of tonics put into the stomach varied from I.5 to 2 c.c.

Our results on the five dogs are summarized in Table VI. The data in this table permit only one conclusion, namely, that these bitters acting in the mouth or in the stomach have no effect on the secretion of gastric juice or on the quantity of food consumed by the normal dog. The slight increase that appears in the case of Dog 5 would probably have been counterbalanced if a larger series of tests had been made. 'The tonics did not produce any changes in the acidity and pepsin concentration.

\section{TABLE VI*}

\begin{tabular}{|c|c|c|c|c|c|}
\hline \multirow{2}{*}{ Dog } & \multirow{2}{*}{ Tonics } & \multirow{2}{*}{$\begin{array}{l}\text { No. of Ex- } \\
\text { PERIMENTS }\end{array}$} & \multicolumn{3}{|c|}{ Gastric JUICE in c.c. } \\
\hline & & & Lowest & Highest & Average \\
\hline Dog I.. & 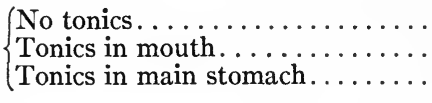 & $\begin{array}{l}\text { I7 } \\
\text { I5 } \\
\text { I5 }\end{array}$ & $\begin{array}{l}\text { I0 } \\
\text { I3 } \\
\text { I0 }\end{array}$ & $\begin{array}{l}33 \\
29 \\
26\end{array}$ & $\begin{array}{l}\text { I9.0 } \\
\text { I8.4 } \\
\text { I8.6 }\end{array}$ \\
\hline $\operatorname{Dog} 2$. & 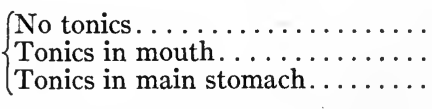 & $\begin{array}{l}\text { I7 } \\
\text { I0 } \\
\text { I0 }\end{array}$ & $\begin{array}{l}3 \\
3 \\
2\end{array}$ & $\begin{array}{l}9 \\
8 \\
7\end{array}$ & $\begin{array}{l}5.1 \\
4.9 \\
4.8\end{array}$ \\
\hline $\operatorname{Dog} 3 \ldots$ & $\left\{\begin{array}{l}\text { No tonics. . . . } \\
\text { Tonics in mouth . } \ldots \ldots \cdots \cdots \\
\text { Tonics in main stomach }\end{array}\right.$ & $\begin{array}{l}\text { I5 } \\
\text { Io } \\
\text { Io }\end{array}$ & $\begin{array}{l}4 \\
5 \\
3\end{array}$ & $\begin{array}{r}\text { I0 } \\
\text { 10 } \\
8\end{array}$ & $\begin{array}{l}7.0 \\
7.2 \\
6.8\end{array}$ \\
\hline $\operatorname{Dog} 4 .$. & $\left\{\begin{array}{l}\text { No tonics. . . . . . . . . . . . . } \\
\text { Tonics in main stomach. }\end{array}\right.$ & $\begin{array}{l}6 \\
6\end{array}$ & $\begin{array}{l}6 \\
5\end{array}$ & $\begin{array}{r}9 \\
\text { II }\end{array}$ & $\begin{array}{l}8.4 \\
8.0\end{array}$ \\
\hline $\operatorname{Dog} 5 \ldots$ & 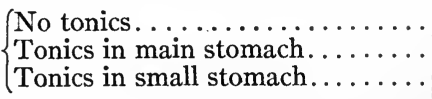 & $\begin{array}{r}\text { ro } \\
9 \\
8\end{array}$ & $\begin{array}{l}8 \\
7 \\
7\end{array}$ & $\begin{array}{l}\text { I6 } \\
\text { I7 } \\
\text { I6 }\end{array}$ & $\begin{array}{l}\mathrm{I} 2.2 \\
\mathrm{I} 4.0 \\
\mathrm{I} 4 . \mathrm{I}\end{array}$ \\
\hline
\end{tabular}

* Dogs with accessory stomachs prepared according to the Heidenhain-Pavlov method. Record of the secretion of gastric juice for the first two hours following a standard meal of lean meat. The variation in the average quantity of gastric juice secreted by the different dogs is due mainly to difference in size of the accessory stomachs.

The possible value of these and other stomachics, especially in digestive disorders, should be investigated by other methods, such as the determination of the actual quantity of food consumed, the 
time required for completion of gastric digestion, etc., with and without the tonics. And the final verdict cannot be given until all possible tests have been applied. But as regards any favorable action of these stomachics on the gastric secretory mechanism in normal men and dogs our results go to show that it is nil, at least when all purely psychic factors are eliminated.

\section{INFLUENCE ON FOOD CONSUMPTION AND ON THE GASTRIC JUICE IN EXPERIMENTAL CACHEXIA}

Hoppe reports that when bitters are given to sick dogs the quantity of gastric juice is increased, and the pepsin and $\mathrm{HCl}$ of the juice is likewise increased. No figures are presented, nor are sufficient accounts of the method used given, so as to enable one to evaluate his conclusions. Moorhead, working in the author's laboratory, produced cachexia in two dogs with Pavlov stomach pouch by repeated excessive hemorrhage, and studied the action of the bitters on the food consumption and the appetite secretion before and after inducing the chronic anemia. The dogs were bled 20 to 30 c.c. per kg. daily until they became permanently listless, weak, and depressed, lost weight gradually, and showed little interest in food or surroundings-in a word, typically cachectic. During the observation period the dogs showed little or no improvement.

\section{TABLE VII}

Quantity of Gastric Secretion in c.c., Cachectic Dogs (Pavlov Stomach)

\begin{tabular}{|c|c|c|c|c|c|c|c|}
\hline \multirow{2}{*}{ SERIES } & \multirow{2}{*}{ No. $T$. } & \multicolumn{3}{|c|}{ Dog I } & \multicolumn{3}{|c|}{ DoG 2} \\
\hline & & Avr. & Mxm. & Mnm. & Avr. & Mxm. & Mnm. \\
\hline \multirow{2}{*}{$\begin{array}{l}\text { Appetite secre- } \\
\text { tion one hour. }\end{array}$} & IO & 0.50 & 2.0 & 0.0 & I. I5 & 5.0 & 0.0 \\
\hline & Io & I. 90 & 4.2 & 0.5 & I. I4 & 2. I & O. I \\
\hline tion one nour.. (Tonics in stomach & IO & I. 06 & $3 \cdot I$ & 0.0 & I. 14 & 2.2 & 0.0 \\
\hline \multirow{3}{*}{$\begin{array}{l}\text { First hour after } \\
\quad \text { feeding........ }\end{array}$} & IO & I.05 & $3 \cdot 9$ & 0.0 & I. 50 & $5 \cdot 9$ & 0.0 \\
\hline & Io & 2.50 & 5.0 & 0.2 & 3. I 5 & 7.7 & O. I \\
\hline & IO & I. 20 & $3 \cdot 5$ & 0.2 & 1.80 & 2.5 & O. I \\
\hline
\end{tabular}

Before the chronic anemia was induced the bitters had no influence on food consumption or on the quality and quantity of 
the appetite gastric juice. During the cachexia the bitters acting in the mouth appeared to increase slightly both the quantity of food consumed, and the quantity and quality of the appetite gastric juice (Table VII). The experiments are not numerous enough to permit final conclusions, even for sick dogs. And it is not clear whether the apparent action of the bitters is a direct one on the appetite mechanism, or in the nature of a condition reflex. The real value of bitter tonics as stimuli to appetite in disease is still an open question, that must be answered by quantitative test methods on the sick.

\section{ACTION ON APPETITE IN CLINICAL CACHEXIA}

Moorhead has completed an extensive series of tests with bitters on five hospital patients with chronic cachexia and poor appetite, using the method of weighing the food consumed, on the theory that if the bitters actually increase appetite this should be revealed in the food consumption. Tonic days were alternated with no-tonic days, so as to exclude any progressive increase or decrease in the appetite from other causes. The results are as follows:

Patient A: given tincture of gentian. The patient was told that the medicine would help his appetite and digestion, and said he felt that it did improve his appetite a great deal. Evidently the subjective faith and hope was greater than the objective results.

Number of control or no-tonic meals.......45

Number of tonic meals................87

Average food consumption, no-tonic meals.... I0. 3 oz.

Average food consumption, tonic meals........II. 3 oz.

Average increase............. I oz. per meal

Patient B: given elixir of iron, quinine, and strychnine. This patient was not told what the medicine was given for, hence he did not know that any attempts were made to improve his appetite.

Number of control or no-tonic meals.......60

Number of tonic meals................. 48 .

Average food consumption, no-tonic meals ... II $8 \mathrm{oz}$.

Average food consumption, or tonic meals.....13.8 oz.

Average increase............. 2 oz. per meal 
Patient C: given elixir of iron, quinine, and strychnine, without being told for what purpose. This patient was a chronic alcoholic and cachectic, with no other definite ailment.

Number of control or no-tonic meals.......33

Number of tonic meals. .............27

Average food consumption, no-tonic meals . . 9.95 oz.

Average food consumption, tonic meals..... ro.84 oz.

Average increase ............ $0.89 \mathrm{oz}$. per meal

Patient D: given elixir of iron, quinine, and strychnine. This patient had chronic myocarditis, and was anemic and cachectic.

Number of control or no tonic meals.........32

Number of tonic meals.................37

Average food consumption, no tonic meals....II.7 oz.

Average food consumption, tonic meals...... I3.2 oz.

Average increase .................. 5 oz.

Patient E: given tincture of gentian. This patient had pernicious anemia, and was very emaciated. He was told that the medicine given him would improve his appetite, and he was very anxious to have his appetite improved.

Number of control or no tonic meals.......22

Number of tonic meals................2

Average food consumption, no tonic meals...19.4 oz.

Average food consumption, tonic meals......23. I oz.

Average increase ............ $\overline{3.7 \mathrm{oz}}$.

The number of different kinds of bitters advocated from time to time as useful in disease is a large one. They are of such varied chemical composition that the only thing they have in common is the bitter taste. Of course, in this discussion, we do not include such substances as quinine or strychnine, or the bitters with alcohol as the main constituent, as these substances have distinct physiologic actions not directly related to appetite, after absorption into the blood.

The bitter tonics are common "home remedies" and favorite "drug-counter prescriptions." They are given to convalescents who would continue to improve, tonic or no tonic, and the tonic, 
not the recuperative power of the patient, gets the credit, or the physician prescribes a more hygienic living and a tonic. The health improves and both physician and patient think the tonic did it.

But even if the bitters have no direct action on gastric secretion and digestion, and no appreciable indirect action on the secretion of gastric juice, may they not be valuable aids in expelling worry and implanting hope and good cheer in the mind of the patient? May they not be an efficient handmaid to psychotherapy? There is no question that the bitter tonics connect up with the popular belief that the potency of a medicine is directly related to its strong (or bad) taste. And the readiness with which these tonics are dispensed nourishes the popular superstition that there is, or must be, a specific drug remedy for every ailment, a superstition that constitutes the chief aid of the medical quack and the patent medicine vender.

The stimulation of certain nerve-endings in the mouth and in the normal gastric mucosa unquestionably contributes to the complex sensation of appetite, and these nerves are stimulated by condiments and flavors of food. Apart from this, the physiologic way of augmenting hunger and appetite is moderation in the food intake or increasing the utilization of the food in the body by outdoor living, fresh air, cold baths, and physical work. If these measures do not improve appetite and hunger, the chances are that the digestive tract is not in a condition to take care of the amount of food demanded by stronger hunger and appetite sensations. 


\section{BIBLIOGRAPHY}

Albertoni, P., and Tullio, P. "Recherches sur les processus digestifs et assimilatifs chez un homme sain avec fistule gastrique et fermenture complète de l' oesophage, Arch. Ital. Biol., LVIII (I9I2), I05.

Albu, A. "Ueber den Aschengehalt einiger Sekrete," Zeitschr. f. exp. Path. u. Pharm., V (Ig08), $\mathrm{r} 7$.

- Grundzïge der Ernährungstherapie, Stuttgart, I 908, 44.

Allen, F. M. "The Starvation Treatment of Diabetes," Jour. Am. Med. Assoc., LXIII (I9I4), 939; Am. Jour. Med. Sci., IV (1915), 480.

Alvarez, W. C. "Functional Variations in Contractions of Different Parts of the Intestine," Am. Jour. of Physiol., XXXV (I9I4), I77.

- "Further Studies on Intestinal Rhythm," Am. Jour. of Physiol., XXXVII (1915), 267.

- "The Motor Functions of the Intestines from a New Point of View," Jour. Am. Med. Assoc., LXV (I9I5), 388.

Bardier, E. "La Faim," in Richet, Dictionnaire de Physiologie, VI (I904), I. Barfurth, D. "Der Hunger als förderndes Princip in der Natur," Arch. f. Mikr. Anat., XXIX (I887), 28.

Beaumont, W. Experiments and Observations on the Gastric Juice and the Physiology of Digestion, Plattsburg, 1833 .

Beaunis. Les Sensations internes, Paris, I889.

Becher, E. "Ueber die Sensibilität der innern Organe," Zeitschr. f. Psych., XI (Ig08), 34r; Arch.f.d. ges. Psych. XV (I909), 356.

Bechterew, W., and Mislavski, N. "Zur Frage von der Innervation des Magens," Neurol. Centralbl., IX (I890), I95.

Benda, Th. "Neurasthenischer Hunger," Deut. med. Woch., XXV (I898), 203. Bender, F. Beitrag zur Lehre von der Magenverdauung. Inaug. Diss., Erlangen, I898, quoted from Malys Jahresb., XXX (I90I), 4 Iо.

Benedict, F. G. A Study of Prolonged Fasting, Carnegie Institution, Washington, I9I 5 .

Bettman, H. W. "The Shape and Position of the Stomach," Philadelphia Mont. Med. Jour., I (1899), I2r.

Bickel, A. "Experimentelle Untersuchungen über die Magensaftsekretion beim Menschen," Berl. kl. Wochenschr., XLII (1905), 60, I44, 869; Deut. med. Wochenschr., XXXII (1906), I323.

—_. "Ueber die Wirkung von Aminosäuren auf die Magensaftsekretion," Internat. Beitr. z. Path. u. Therap. d. Ernährungs., V (1913), 75.

Biedermann, W. "Die Ernährung der Einzelligen (Protozoa)," Handbuch $d$. Vergleich, Physiol., II (1910), 273. 
Biedl, A. "Die Innere Sekketion," —_ II (Igr 3), 286.

Boas, J. "Ueber Nausea," Berl. klin. Wochenschr., XLVI (г909), г гог.

—. Die Diagnostik u. Therapie der Magenkrankheiten, Leipzig, I9г r.

Bogen, H. "Experimentelle Untersuchungen über psychische und assoziative Magensaftsekretion beim Menschen," Arch.f. d. ges. Physiol., CXVII (I907), I50.

Boldyreff, W. "Le Travail périodique de l'appareil digestif en dehors de la digestion," Arch. d. Sc. Biol., XI (1905), I.

_. "Einige neue Seiten der Tätigkeit des Pancreas," Ergebnisse der Physiologie, XI (I9II), I86.

- "The Self-Regulation of the Acidity of the Gastric Contents and the Real Acidity of the Gastric Juice," Quart. Jour. Exper. Physiol., VIII (Igr4), I.

Bönninger, M. "Zur Diagnose des Ulcus Ventriculi," Berl. klin. Wochenschr., XLV (1908), 396.

Boring, E. G. "Processes Referred to the Alimentary Tracts," etc., Psych. Review, XXII (I9I5), 306.

- "The Sensations of the Alimentary Canal," Am Jour. Psych., XXVI (I9I 5), I.

- "The Thermal Sensitivity of the Stomach," Am. Jour. Psych., XXVI (I9r5), 485 .

- "Processes Referred to the Alimentary and Urinary Tractsa Qualitative Analysis," Psych. Review, XXII (I9I 5), 306.

Borissow, W. "Ueber die Bedeutung der Bitterstoffe für die, Verdauung," Arch.f. exp. Path. u. Pharm., LI (1904), 363.

Bostock, J. An Elementary System of Physiology, London, I836, 593.

Brachet, J. L. Recherches sur les fonctions d. syst. nerv., Paris, I837.

Brodie, G. T., and Russel, A. E. "On Reflex Cardiac Inhibition," Am. Jour. of Physiol., XXVI (Igoo), 92.

Brunemeier, E. H., and Carlson, A. J. "Reflexes from the Intestinal Mucosa to the Stomach," Am. Jour. of Physiol., XXXVI (I9I4), I9I.

Bulawinzew. "Ueber psychischen Magensaft beim Menschen," Ruski Wratsch. (1903), No. I7, quoted from Bioch. Centralbl., X (I903), 593.

Bunge, G. Lehrb. der physiol. Chem., I894, II3.

Busch, W. "Beitrag zur Physiologie der Verdauungsorgane," Arch.f. Pathol. Anat., XIV (I862), I40.

Cade, A., and Latarjet, A. "Realization pathologique du petit estomac de Pavlov," Jour. de Physiol. et Path., VII (1905), 221.

Cannon, W. B. "The Motor Activities of the Stomach and Small Intestines after Splanchnic and Vagus Section," Am. Jour. of Physiol., XXVI (I906), 429 .

- "The Acid Control of the Pylorus," Am. Jour. of Physiol., XX (I907), 283 . 
Cannon, W. B., and Lieb, C. W. "The Receptive Relaxation of the Stomach," Am. Jour. of Physiol., XXVII (I9II), xiii.

Cannon, W. B. The Mechanical Factors in Digestion, New York, I9г I.

- A Consideration of the Nature of Hunger. Harvey Lectures, Philadelphia, I9I I, p. I30.

- "The Nature of Gastric Peristalsis," Am. Jour. of Physiol., XXIX (1915), 250.

Cannon, W. B., and Washburne, A. L. "An Explanation of Hunger," Am. Jour. of Physiol., XXIX (I912), 44I.

Carlson, A. J. "The Condition of the Digestive Tract in Parathyroid Tetany," Am. Jour. of Physiol., XXX (1912), 309.

- "The Character of the Movements of the Empty Stomach in Man," Am. Jour. of Physiol., XXXI (I9I2), I5I.

- "The Relation between the Contractions of the Empty Stomach and the Sensation of Hunger," Am. Jour. of Physiol., XXXI (I913), I75.

- "The Gastric Hunger Contractions Inhibited Reflexly from the Mouth," Am. Jour. of Physiol., XXXI (I9I3), 2 I 2.

- "The Influence of the Gastric Hunger Contractions on the Cardiac and Vasomotor Centers, and on the Reflex Excitability of the Spinal Cord," Am. Jour. of Physiol., XXXI (1913), 318.

- "The Influence of Stimulation of the Gastric Mucosa on Gastric Hunger Contractions," Am. Jour. of Physiol., XXXII (I913), 245.

- "A Study of the Mechanism of the Hunger Contractions of the Empty Stomach by Experiments on Dogs," Am. Jour. of Physiol., XXXII (1913), 369.

- "The Inhibitory Reflexes from the Gastric Mucosa," Am. Jour. of Physiol., XXXII (1913), 389.

- . "The Tonus and Hunger Contractions of the Empty Stomach during Parathyroid Tetany," Am. Jour. of Physiol., XXXII (I913), 398.

- "The Hunger Contractions of the Empty Stomach during Prolonged Starvation (Man, Dog)," Am. Jour. of Physiol., XXXIII (I9I4), 95.

- "A Method of Obtaining Successive Contrasts of the Sensations of Hunger and Appetite," Am. Jour. of Physiol., XXXIII (I9I4), xv.

- "The Nervous Control of the Gastric Hunger Mechanism," Am. Jour. of Physiol., XXXIV (1914), I55.

- "The Supposed Action of Bitter Tonics on the Secretion of Gastric Juice in Man and Dog," Jour. Am. Med. Assoc., LXIV (I9I5), I5.

- "The Secretion of Gastric Juice in Man," Am. Jour.' of Physiol., XXXVI (1915), 50.

- "On the Chemistry of Normal Human Gastric Juice," Am. Jour. of Physiol., XXXVIII (I915), 248. 
Carlson, A. J., Orr, J. S., and McGrath, G. W. "The Hunger Contractions of the Stomach Pouch Isolated according to the Method of Pavlov," Am. Jour. of Physiol., XXXIII (I9I4), II9.

Carlson, A. J., and Luckhardt, A. B. "The Condition of the Esophagus during the Gastric Hunger Contractions," Am. Jour. of Physiol., XXXIII (I9I4); I26.

Carlson, A. J., Van de Erve, J., Lewis, J. H., and Orr, J. S. "The Action of the So-Called Stomachics or Bitters on the Hunger Mechanism," Jour. of Pharm. and Exp. Therap., VI (I9I4), 209.

Carlson, A. J., and Lewis, J. H. "The Influence of Smoking and of Pressure on the Abdomen on the Gastric Hunger Contractions," Am. Jour. of Physiol., XXXIV (I9I4), I49.

Carlson, A. J., and Ginsburg, H. "The Gastric Hunger Contractions of the Newborn," Am. Jour. of Physiol., XXXVII (1915), 29.

Carlson, A. J., and Braafladt, L. H. "The Sensibility of the Gastric Mucosa, Am. Jour. of Physiol., XXXVI (I9I4), I 53.

Carlson, A. J., and Ginsburg, H. "The Tonus and Contractions of the Empty Stomach in Infants with Congenital Pyloric Stenosis and Pylorospasm," Am. Jour. of Physiol., XXXIX (I9I6), 3 Iо.

Carrington, . Vitality, Fasting and Nutrition, New York, 1908.

Chittenden, R. The Nutrition of Man, New York, I907, I64.

Cohn, J. "Ueber den Einfluss massiger Korpenbesuchung auf die Verdauung," Deutsch. Arch.f. klin. Med., XLIII (г888), 239.

Cole, L. G. "The Complex Motor Phenomena of Various Types of Unobstructed Gastric Peristalsis," Arch. of Roentg. Ray, XVI (I9II), 242, 259.

Cushny, A. R. Pharmacology and Therapeutics, Philadelphia, I9ı, 55.

Darwin, E. Zoonomia, London, I80I, III, 222.

Dietlan, H. "Ergebnisse des medizinischen Roentgensverfahrens für dic Physiologie," Ergeb. d. Physiol., XIII (I9I3), 47.

Dubois, S. "Traitement de l'anorexie hystérique par les injections hypodermiques de morphine," Prog. Med., III (I896), i I9.

Ducceschi, V. "Sulle funzioni Motrici dello Stomaco," Arch. per lc Sci. Med., XXI (1897), I54, quoted from Luciani, Physiologic des Menschen, I905, II, I63.

—. "Sugli effetti della sezione dei nervi gastrici," Archivo di fisiologica, VIII (I9I0), 579 .

Edelmann, . Die Bewegungen des Magens, etc., Thesis, St. Petersburg, 1906, quoted from Magnus.

Ehrlich, F. "Ist die schmerzhafte Magenleere eine nervöse Erkrankung?" Münch. med. Wochenschr., LI (I904), 882.

Einhorn, M. "Sitophohia, Inanition and Their Treatment," Am. Jour. Mcd. Sc., CXXVI (1903), 228. 
Elsesser, O. J. "The Effect of Experimental Stenosis of the Pylorus on the Tonus and Contractions of the Empty Stomach," Am. Jour. of Physiol., XXXIX (I9I 5), 303.

Elsner, H. "Die Motilität des Magens bei Achylia Gastrica," Deut. med. Wochenschr., XXX (I904), 42.

Enriquez and Hallion. "Sur l'excitation du péristaltisme intestinal par des extracts d'organes," Compt. rend. soc. biol., LXXI (I9I I), 488.

Eppinger, H., and Hess, L. Vagotonia, New York, I9I 5.

Ewald, J. R. Klinik der Verdaungskrankheiten, II (I893), 457.

Fawcett, Rahe, Hackett, and Rogers. "The Effects of Aqueous Extracts of Organs on the Contractions of Unstriated Muscle Fibres," Am. Jour. of Physiol., XXXIX (1915), I 54.

Forsell, G. "Ventrikelrörelserna hos Människan," Nord. Med. Arch., XI (I9II), 64 .

Forster, J. "Versuche ueber die Bedeutung des Aschebestandtheile in der Nahrung," Zeitschrift f. Biol., IX (I873), 297.

Foster, B. N., and Lambert, A. V. S. "Some Factors in the Physiology and Pathology of Gastric Secretion," Jour. Exp. Med.; X (I908), 820.

Foster, M. Textbook of Physiology, London, I891, IV, 285.

Friedenthal, H. "'Beiträge zur Kentniss der Fermente," Arch. f. Physiol., (1900), I8Ir.

Fuchs, D. "Ueber den Einfluss des Hungerns auf die Ausscheidung von Aminosäuren," Zeitschr.f. physiol. Chem., LXIX (igro), 49r.

Gaglio, G. "Ipersecrizione gastrica sperimentale," Arch. per le Sc. Med., XXII (1902), quoted from Jahresb. d. ges. Med., II (r go2), I96.

Gemelli, A. "Saggia di una tioria biologica sulla genesi della fame," Mem.d. Pout. Acend. Rom. d. Mo., XXV (1907), 249.

Gibson, A. E. "The Physiology of Hunger," Med. Rec., LXV (1904), 574.

Ginsburg, H., Tumpowsky, I., and Carlson, A. J. "The Onset of Hunger in Infants after Feeding," Jour. Am. Med. Assoc., LXIV (I9I5), I822.

Glaessner, K., and Kreuzfuchs, S. "Ueber den Pylorospasmus," in ünch. med. Wochenschr., LX (1913), 582.

Glücksmann, G. "Anskulatorische Phänomene am Intestinal-Traktus und ihre diagnostische Verwertbarheit," Deut. med. Wochenschr., XXXVII (I9II), 590.

Goltz, F. "Der Hund ohne Grosshirn," Arch.f. d. ges. Physiol., LI (1892), 570. Goodall, J. S. "Appetite: An Attempted Analysis of the Psychic Factor," Brit. Med. Jour., II (I908), 586.

Haller, A. Opera Minora, Lausanne, I 763, I, 356.

-. "Fames et Sitis," Elementa Physiologiae, I776, VI, I85.

Hardt, L. L. " "The Secretion of Gastric Juice in Cases of Gastric and Duodenal Ulcers," Am. Jour. of Physiol., XL (1916), 3 I4. 
Head, H., and Holmes, G. "Sensory Disturbances from Cerebral Lesions," Brain, XXXIV (I9II), rog.

Head, Rivers, and Sherren. "The Afferent Nervous System from a New Aspect," Brain, XXVIII (1905), 99.

Heilner, E., and Poesgen, F. "Eiweisspaltender Fermente im Blute bei vorgeschrittenen Hunger," Münch. med. Wochenschr., LXI (I9I4), 402.

Hertz, A. F., Cook, F., and Schlessinger, E. G. "The Sensibility of the Stomach and Intestines in Man," Am. Jour. of Physiol., XXXVII.(Igo8), $48 \mathrm{r}$.

Hertz, A. F. The Sensibility of the Alimentary Canal, London, Igrr.

Heubner, W., and Rieder, R. "Ueber die Wirkung der Bitterstoffe auf die Resorption," Therap. Monatschr., XXXIII (I909), 3 го.

His, W. "Studien über Form und Lagerung des menschlichen Magens," Archiv. f. Anat., 1903, 345.

Hodge, C. F., and Aikens, H. A. " "The Daily Life of a Protozoan," Am. Jour. of Psych., VI (I893), 524.

Hooker, R. D. "The Effect of Carbon Dioxide .... on the Muscular Tone .... of the Alimentary Canal," Am. Jour. of Physiol., XXXI (I9I2), 47.

Hopf, H. "Ueber den hemmenden und erregenden Einfluss des Vagus auf den Magen des Frosches," Zeitschr.f. Biol., LV (r 1 r I), 409.

Hoppe, Th. "Experimentelle Untersuchungen ueber die Wirkung einiger Stomachica auf die Magenssftsekretion," Berl. kl. Wochenschr., XLII (1905), 1038.

Hornborg, A. F. "Beiträge zur Kentniss der Absonderungsbedingungen des Magensaft beim Menschen," Skand. Arch. f. Physiol., XV (r904), 209.

Howe, P. E. " "General Aspects of Fasting," Bioch. Bull., II (I9r 2), 90.

Hudek u. Stigler. "Radilogische Untersuchungen über den Zusammenhang zwischen der Austreibungszeit des normalen Magens und Hungergefühl," Arch.f.d.ges. Physiol., CXXXIII (I9ro), $\mathrm{r}_{45}$.

Jacobi, A. "Gastric Ulcer," Trans. Congr. of Am. Phys. and Surg., VII (1907), r 2 r.

Jennings, H. S. "The Psychology of a Protozoan (paramecium)," Am. Jour. of Psych., X (r899), 503.

- Contribution to the Study of the Behavior of the Lower Organisms, Carnegie Institution of Washington, I904.

Johansson, Landergren, Sonden, and Tigerstedt, "Beiträge zur Kentniss des Stoffwechsels beim hungernden Menschen," Skand. Arch. "f. Physiol., VII (I896), 33 .

Jones, A. A. "Hunger Pain," Jour. Am. Med. Assoc., LIX (rgr2), II 54.

Kaestle, Rieder, and Rosenthal. "Ueber Roentgenkinimatographie innerer Organe des Menschen," Mïnch. Mcd. Wochcnschr., LVI (I909), 280. 
Kast, L., and Meltzer, S. J. "On the Sensibility of the Abdominal Organs," Med. Rec., LXX (1906), гог7; Mitt. a. d. Grenzgeb. Med. u. Chir., XIX (I909), 586.

Kaznelson, Helene. "Scheinfütterungsversuche am Menschen," Arch. f. d. ges. Physiol., CXVIII (1907), 327.

Keeton, R. W. "The Secretion of Gastric Juice during Parathyroid Tetany," Am. Jour. of Physiol., XXXIII (I9I4), 25.

Keeton, R. W., and Koch, F. C. "The Distribution of Gastrin in the Body," Am. Jour. of Physiol., XXXVII (I9I5), 48I.

Keith, A. "A New Theory of the Causation of Enterostasis," Lancet, II (I915), 37 I.

Kendall, I. K. "Studies in Bacterial Metabolism," Jour. of Inf. Dis., XIII, XV (I9I3-I4), Jour. Biol. Chem., XIII, XV; Jour. Am. Chem. 8vo., XXXV, XXXVI (r9r3-r4).

Kerb, W. "Ueber den Nährwert der im Wasser gelösten Stoffe," Internat. Rev. d. g. Hydrob. u. Hydrogr., III (I9I0).

King, Jessie L., and Connet, Helene. "The Gastric Hunger Contractions of the Normal and the Decerebrated Guinea-Pig," Am. Jour. of Physiol., XXXIX (I915), I23.

Klee, P. "Der Einfluss von Vagusreizung auf den Ablauf der Verdauungsbewegungen," Arch.f.d. ges. Physiol., CXLV (1912), 557.

Knapp, M. I. "The Nature and Cause of Hunger, Appetite, and Anorexia," Amer. Medicine, X (1905), 353.

Kniep, H. "Untersuchungen über die Chemotaxis von Bakterien," Jahrb. f. Wiss. Bot., XLIII (1906), 215.

Krehl, L. The Basis of Symptoms. Principles of Clinical Pathology, Philadelphia, 1916, 257 .

Krizenecky, J. "Ueber die beschleunigende Wirkung des Hungern auf die Metamorphose," Biol. Zentralbl., XXXIV (I9r4), 46.

Kusano, S. "Studies on the Chemotactic and Other Related Reactions of Swarmspores of Myxomycetes," Jour. Coll. Agr. Imp. Univ., Tokyo, II (I909), I.

Kussmaul, A. "Die peristaltische Unruhe des Magens," Volkmanns Saml. $k l$. Vortr., I880, I8r.

Langfeld, H. S. "The Psycho-physiology of a Prolonged Fast," Psych. Monographs, XVI (I9I4), No. 6.

Lavenson, R. S. "Observations on a Child with a Gastric Fistula," etc., Arch.f. Int. Med., IV (1909), 27 r.

Lehmann, Müller, Munk, Senator, and Zuntz. "Untersuchungen an zwei hungernden Menschen," Arch.f. Path. Anat. Suppl., CXXXI (г893), I.

Lehmann, M. "Zur Frage des osmotischen Drucks menschlicher Mageninhalte," Zeitschr.f. exper. Path. u. Pharm., III (1906), 559. 
Lennander, K. G. " "Leibschmerzen, ein Versuch, einige von ihnen zu erklären," Grenzgeb.d. Med.u. Chir., XVI (I906), 24.

Leo, H. "Ueber Bulimia," Deut. med. Wochenschr., XV (1889), 581.

Levi, L. "Des viciations de la faim bulbaire," Compt. Rend. Soc. Biol., LVIII (1905), 7 ro.

Lipschütz, A. "Die Ernährung der Wassertiere durch die gelösten organischen Verbindungen der Gewässer," Ergeh. d. Physiol., XIII (I913), I.

Lister, A. "Notes on the Ingestion of Food Material by the Swarm-Cells of Mycetozoa," Jour. Linn. Soc., XXV (189o), 435.

Loeb, J. "Chemotropismus," Handb. d. Vergl. Physiol., IV (I91 2), 504.

Lombard, W. P. "The Variations of the Normal Knee-Jerk and Their

Relation to the Activity of the Central Nervous System," Am. Jour. of Psych., I (1887), 5.

Longet, F. A. Traité de physiologie, Paris, I868, I, 23.

Lönnquist, B. "Beiträge zur Kentniss der Magensaftabsonderung," Skand. Arch.f. Physiol., XVIII (1906), 323.

Luciani, L. Das Hungern, Leipzig, I89o.

- "Sulla genesi delle sensazioni della fame e della sete," Archivo de Fisiologica, III (1906), 542.

- Physiologie des Menschen, Jena, I9II, IV, 64.

Luckhardt, A. B. "The Cause of the Polyphagia of Pancreatic Diabetes," Am. Jour. of Physiol., XXXIII (r914), 3 I5.

Luckhardt, A. B., and Carlson, A. J. "On the Chemical Control of the Gastric Hunger Mechanism," Am. Jour. of Physiol., XXXVI (I9I4), 37.

- "The Influence of Dreaming on the Gastric Hunger Contractions," Am. Jour. of Physiol., XXXIX (r9r6), 330.

Luckhardt, A. B., and Hamburger, W. W. "Note on the Movements of the Empty Stomach in Certain Pathological States in Man," Jour. Am. Med. Assoc., LXVI (rgr6), r83r.

Ludwig, C. Physiologie des Menschen, Leipzig, I858, II, 584.

Lunin, N. "Ueber die Bedeutung des anorganischen Salzes für die Ernährung des Tieres," Zeitschr. f. Physiol. Chem., V (188r), 3 г.

Lusk, G. "The Influence of Cold Baths on the Glycogen Content of Man," Am. Jour. of Physiol., XXVII (I9rI), 427.

MacCallum, H. A. "Gastric Neurasthenia," Brit. Med. Jour., II (I906), Iо3 I. Mackinzie, J. Symptoms and Their Interpretations, London, I909, I 25.

Magendie, F. Lehrbuch der Physiologie, Tübingen, 1826, II, I9.

Magnus, R. "Die Bewegungen des Verdaungskanals," Ergeb. d. Physiologie, VII (1908), 30.

Mangold, E. "Die funktionellen Schwankungen der Motorischen Tägigkeit des Raubvogelmagens," Arch.f. d. ges. Physiol., CXXXIX (г9i I), io.

Mangold u. Felldin. "Ueber den Einfluss verschiedenartiger Fütterung auf die Bewegungen des Hünermagens," Zentralbl.f. Physiol., XXIII (I909), 302. 
Mariott, W. M. "The Blood in Acidosis," Jour. Biol. Chem., XVIII (I9I4), 507.

Marshall, C. F. "A Fatal Case of Anorexia Nervosa," Lancet, I (1895), I 49. Mayer, S. "Zur Pathologie des Hungersgefühls," Monatschr. f. Psych. u. Neurol., XXVI, 232.

McCollum, E. V. "The Nature of the Repair Processes in Protein Metabolism," Am. Jour. of Physiol., XXIX (I9II), 2 I 5.

Meisl, A. "Ueber das Wesen des.Appetits," Wien. kl. Rundschau, XVII (I903), 375 .

Meltzer, S. J., and Auer, J. "Peristaltic Rush," Äm. Jour. of Physiol., XX (1907), 259.

Mendel, L. B. "The Physiology of Hunger," Diet. and Hyg. Gaz., IX (1893), 281 .

Menten, M. L. "The Acidity of Normal Gastric Juice in Man," Jour. Biol. Chem., XXII (1915), 34I.

Meumann, E. "Zur Frage der Sensibilität der innern Organe," Arch. f. $d$ ges. Psych., IX (1907), 26; XIV (I909), 279; XVI (1910), 228.

Miller, F. R. "Blutdruckveränderungen bei Riezung des Magenvagus," Arch.f.d.ges. Physiol., CXLIII (I9I I), I.

- On Visceral Sensation, Especially That of the Stomach, Thesis, Munich, I9II.

Milne-Edwards. Leçons sur la physiologie, XIII (I878), 490.

Mitchell, J. F. "The Sensibility of the Peritoneum and the Abdominal Viscera," Jour. Am. Med. Assoc., LVII (I9II), 709.

Molhant, M. "Le nerf vague," Le Nevraxe, XIII-XV (I.9Io-I3).

Moorhead, L. D. "Further Studies on the Action of Bitter Tonics on the Secretion of Gastric Juice," Jour. of Pharm. and Exp. Therap., VII (1915), 577 .

Morat, J. P. "Sur l'innervatier motrice de l'estomac," Lyon Medical, XL (I882), 289, 335; Arch. d. Physiol., V (I893), I42.

Morgulis, S. "The Influence of Protracted and Intermittent Fasting upon Growth," Amer. Naturalist, XLVII (19I3), 477.

-. "Studies on Fasting Flounders," Jour. Biol. Chem., XX (I915), 37. Morgulis, Howe, and Hawk. "Studies on Tissues of Fasting Animals," Biol. Bull., XXVIII (I9I 5), 397.

Moritz, F. "Studien ueber die Motorische Thätigkeit des Magens," Zeitschr.f. Biol., XXXII (1895), 313; XLII (1901), 565.

Moynihan, B. G. A. Duodenal Ulcer, London, I910, Iоr.

Müller, J. Handbuch der Physiologie des Menschens, Coblenz, I844, I, 398.

Müller, L. R. "Ueber die Hungerempfindung," Deut. med. Wochenschr., XLI (I915), I297.

Neumann, A. "Ueber die Temperaturempfindlickkeit des Magens," Wien. $k l$. Wochenschr., XIX (1906), 923. 
Neumann, A. "Ueber die Sensibilität der inneren Organe," Centralbl. f. d. Grenzgeb. Med.u. Chir., XIII (I9Iо), 40I, etc.

Nicolai, W. Ueber die Entstehung des Hüngergefühls, Inaugural Dissertation, Berlin, I892.

Nisser, E., u. Brauning, H. "Ueber normale und ueber vorzeitige Sättigung," Mïnch. med. Wochenschr., LVIII (I9II), 1955.

Nyström, G. "Ueber die Sensibilität der Bauchorgane," Gernzgeb. Med: u. Chir., XXI (I909), I25.

Okada, S. "On the Contractile Movement of the Gall Bladder," Jour. of Physiol., L (I9I5), 42.

Park, M. Travels in the Interior of Africa, Philadelphia, 1800.

Parker, G. H. "The Reversal of the Effective Stroke of the Labial Cilia of Sea-Anemones by Organic Substances," Am. Jour. of Physiol., XV (I905), I.

Patterson, T. L. "The Variations in the Hunger Contractions of the Empty Stomach with Age," Am. Jour. of Physiol., XXXIII (I9I4), 473.

- "The Cause of the Variations in the Gastric Hunger Contractions with Age," Am. Jour. of Physiol., XXXVII (I9I 5), 3I6.

- "The Hunger Contractions of the Empty Stomach in Reptilia and Amphibia," Am. Jour. of Physiol., XXXVIII (igr6), I40.

Pavlov, I. P. The Work of the Digestive Glands, London, rgro.

Perthes, G. "Zur chirugischer Behandlung des Magenschwürs," Deut. Zeitschr.f. Chir., CXXIX (Igr4), 5II.

Pfaundler,.M. "Ueber einer neuen Methode zur klinischen Funktionsprüfung des Magens und deren physiologischen Ergebnisse," Deut. Arch. f. kl. Med., LXV (I900), 255.

Pfeffer, W. "Ueber chemotactischer Bewegungen von Bacterien, Flagellaten und Volvocineen," Unters. Bot. Inst., Tübingen, II (1888), 582.

Pick, A. "Die Sensibilitätsneurosen des Magens," Wien. kl. Wochenschr., XIV (I90r), 8r 7,849 .

- "Ueber Magenschmerz," Wien. kl. Rundschau, XX (ı906), I.

"Die Behandlung der Appetitlosigkeit mit besonderer Berücksichtigkeit ihrer nervösen Formen," Wien. kl. Wochenschr., LXIII (I9I3), 105.

Ploenies, W. "Die Reizerscheinungen d. Mundes, d. Verhalten d. Appetits und Heisshungererscheinungen b. d. Erosionen und Geschwüren des Magens," Zeitschr.f. kl. Med., LIX (Igo6), 88.

Polanyi, M. "Untersuchungen über die Veränderung der physikalischen und chemischen Eigenschaften des Blutserums während des Hungerns," Bioch. Zeitschr., XXXIV (I9II), I92.

Polimanti, O. "Ueber die Ursache und die biologische Bedeutung des Hungern," Naturw. Wochenschr. (N. F.) X (I9II), 593. 
Pütter, A. "Die Ernährung der Wassertiere," Zeitschr. f. allg. Physiol., VII (I907), 22I, 283.

Rehfus, Bergeims, and Hawk. "The Residuum Found in the Empty Stomach," Jour. Am. Med. Assoc., LXIII (I9I4), II ; LXV (I915), I021.

Rehfus, N. E., and Hawk, P. B. "Secretion of Gastric Juice of a Constant Acid Concentration in Man," Jour. Am. Med. Assoc., LXIII (I9I4), 2088.

Reichardt, M. "Ueber normale und krankhafte Vorgänge in der Hirnsubstanz," Arbeiten aus der psychiatrischen Klinik in Würzburg, Jena, VII, (1914), 690.

Reichmann, N. "Experimentelle Untersuchungen über den Einfluss der bitteren Mittel auf die Funktion des gesunden und kranken Magens," Zeitschr.f. kl. Med., XIV (I888), I 77 .

Reiss, A. Ueber Polyphagia. Freiburg (C. Lehmann), 1896.

Rhumbler, L. "Physikalische Analyse von Lebenserscheinungen der Zelle," Arch.f. entw. Mech:, VII (I898), I04.

Riegel, F. "Ueber die continuerlische Magenaftsekretion," Deut. med., Wochenschr., XIX (I893), 735, 769 .

Ritter, C. "Zur Frage der Sensibilität der Bauchorgane," Centralbl.f. Chir., XXXV (I908), 609.

Rogers, F. T. "The Contractions of the Rabbit's Stomach during Hunger, Am. Jour. of Physiol., XXXVI (1915), I83.

- "The Hunger Mechanism in Birds," Proc. Soc. Exp. Biol. and Med., XIII (I9I6), II9.

Rogers, F. T., and Hardt, L. L. J. "The Relation of the Digestion Contractions of the Filled to the Hunger Contractions of the Empty Stomach," Am. Jour. of Physiol., XXXVIII (19r5), 274.

Rosemann, R. "Die Eigenschaften und die Zusammensetzung des durch Scheinfütterung gewonnenen Hundemagensaftes," Arch.f.d.ges. Physiol., CXVIII (I907), 467.

Rossi, G. "Sulla meccanica dell'apparato digerente del pollo," Arch. di fisiologica, II (1905), 375 .

Roux, J. "La faim. Etude physio-psychologique," Bull. Soc. Anthrop., Lyon, XVI (I897), 409.

- Maladies du tube digestive, Paris, 1907, 375.

Sadowen, A. "Ueber das Hungern des Menschen," Malys Jahresber., XVIII (I889), 288.

Schaeffer, A. A. "Selection of Food in Stentor," Jour. of Exp. Zoöl., VIII (I910), 75 .

Scheunert, H. "Ueber den Einfluss der Körpenbewegung auf die Verdauung und Nährstoffsabsorption," Arch.f.d.ges. Physiol., CIX (I905), I45.

Schiff, M. Physiologie de la digestion, Florence, 1867, 29, 57. 
Schlessinger, H. "Beitrag zur Kentniss der Hungergefühls," Wien. kl. Wochenschr., VI (1893), 566.

Schlossmann, A. "Untersuchungen und Erwägungen ueber den Hunger," Deut. med. arzt. Zeitschr., XLIII (I9I4), I70.

Schmidt, J. E. "Beitrag zur Frage der Magensensibilität," Grenzgeb. Med. u. Chir., XIX (I908), 278.

Schoumow-Simanowski, E. O. "Ueber den Magensaft und das Pepsin bei Hunden," Arch.f. exp. Pathol. u. Pharm., XXXIII (I894), 336.

Schüle, A. "Zur Kentniss von der Zusammensetzung des normalen Magensaftes," Zeitschr.f. kl. Med., XXXIII, XXXV, 543.

—_. "In wie weit stimmen die Experimente von Pawlow am Hunde mit den Befunden am normalen menschlichen Magen ueberein"? Deut. Arch. f. kl. Med., LXXI (IgOI), Irr.

Schüller, A. "Die Erkrankungen der Zirbeldrüse," Handb. d. Neurol., II (I913), 337 .

Sedillot, C. E. Du nerf vague et ses fonctions, Paris, I829.

Seland, V. "Ueber die Nachwirkung der Nahrungsentzieung auf die Ernährung," Biol. Centralbl., VI (1887), I45, etc.

Sherrington, C. S. "Experiments on the Value of Vascular and Visceral Factors for the Genesis of Emotion," Proc. Roy. Soc., LXVI (I goo), 390.

Shibata, K. "Untersuchungen ueber die Chemotaxis der PtridophytenSpermatozoiden," Jahrb.f. Wiss. Bot., XLIX (IgI I), I.

Sick, K. "Untersuchungen ueber die Saftabsonderung und die Bewegungsvorgänge im Fundus und Pylorusteil des Magens," Arch. f. kl. Med., LXXXVIII (I906), I69.

Sinclair, A. The Fasting Cure, New York, I 1 I r.

Smith, F. Veterinary Physiology, London, 1907, 327.

Soemmering, S. T. De Corporis humani Fabrica, I794, VI, 237.

Sommerfeld, P. "Zur Kentniss der Sekretion des Magnus beim Menschen," Arch.f. Anat. u. Physiol., Suppl., 1905, 455.

Spencer, Meyer, Rehfus, and Hawk. "Direct Evidence of Duodenal Regurgitation and Its Influence upon the Chemistry and Function of the Normal Stomach," Am. Jour. of Physiol., XXXIX (rgr6), 459.

Sternberg, W. "Die Appetitlosigkeit," Zentralbl.f. Physiologie, XXII (I908), 233,653 .

—. "Die Physiologische Grundlage des Hungergefühls," Zeitschr. f. Sinnes-Physiologie, XLV (1910), $7 \mathrm{I}$.

—. "Der Appetit in der exakten Medizin," Zeitschr.f. sinnes-Physiologie, XLV (I9II), 433.

- Das Nahrungsbedürfnis, der A ppetit und der Hunger, Leipzig, I913.

Stiller, B. "Die Pathologie des Appetits," Arch. f. Verdaunngskr., XXI (1915), 23. 
Stöhr, A. Ascese und Heilkunde; Handbuch der Pastoralmedicin, Freiburg, I $878,420$.

Stübel, H. "Der Erregungsvorgang in der Magenmuskulatur nach Versuchen am Frosch- und Vogelmagen," Arch.f.d.ges. Physiol., CXLIII (I9 I I), 38г.

Takahashi, M. "Die Abhängigkeit der Magenentleerung vom Allgemeinzustand des Nervensystems," Arch.f. d. Physiol., CLIX (I9r4), 389.

Tiedemann, F. Physiologie des Menschens, I836.

Tobler, L. "Ueber die Eiweissverdauung im Magen," Zeitschr. f. Physiol. Chem., XLVI (1905), 185 .

Troller, J. "Ueber Methoden zur Gewinnung reiner 'Magensekretion, Zeitschr.f. kl. Med., XXXVIII (1898), I83.

Turro, R. "Die physiologische Psychychologie des Hungers," Zeitschr. $f$. Sinnes-Physiologie, XLIV (1910), 330; XLV (191r), 217, 327.

Umber, F. "Die Magensaftsekretion des Menschen," Berl. kl. Wochenschr., XIX (1905), 29.

Valenti, A. "Sur la genèse des sensations de faim et de soif," Arch. Ital. di Biol., LIII (1910), 97.

Vanlair, C. "La faim," Rev. d. Belg., XXXV (1903), г99, 320.

Verhagen, A. "De la variabilité de l'acidité gastrique à l'état normal," La Cellule, XIV (I898), 29.

Vertes, O. "Ueber das wechselseitige Verhältnis zwischen den weiblichen Geschlechtsorganen und den Erkrankungen des Magens," Monatschr. f. Geburtsh. u. Gynäk., XXXII (I9II), I 28.

Verworn, M. Psycho-physiologische Protisten Studien, Jena, I889, I46.

Vierordt, H. Grundriss, der Physiologie, Tübingen, I871, 433.

Voit, C. von. "Hunger- und Durstgefühl," Hermanns Handbuch der Physiologie, $\mathrm{V}(\mathrm{I} 88 \mathrm{I}), 560$.

Voit, E. "Ueber die Ursache der Zunahme der Eiweisszersetzung des hungernden Tieres," Zeitschr.f. Biol., XLI (I90I), 550.

Vorkastner, W. "Organen Neurosen," Lemandowskys Handbuch d. Neurologie, Igr 3, V, No. 4,7 .

Wallengren, H. "Inanitionserscheinungen der Zelle," Zeitschr. f. allg. Physiologie, I (1902), 67.

Weber, E. H. Wagners Handwörterbuch d. Physiologie, 1846, III, 497.

Weber, S. "Ueber Hungerstoffwechsel," Ergeb. d. Physiol., I (I902), 702.

Weiland, W. "Zur Kentniss der Entstehung der Darmbewegung," Arch.f.d., Physiol., CXLVII (I912), I7 I.

Weygandt, W. "Ueber die psychische Wirkungen des Hungers," Münch. med. Wochenschr., XLV (I898), 385.

- "Ueber die Beeinflüssung geistiger Leistungen durch Hungern," Kraepelin. Psych. Arbeiten, IV (1901), 45.

Wolff, W. Der Bewegungen des Duodenums, Dissertation, Giessen, 1902. 


\section{I6 CONTROL OF HUNGER IN HEALTH AND DISEASE}

Wundt, W. Physiologisches Psychologie, I902, II, 43.

Yukawa, G. "Ueber die Salzsäuremenge im physiologischen Magensafte der Japaner," Arch.f. Verdauungskr., XIII (1907), 523, 535.

Zimmerman, R. "Experimentelle Untersuchungen ueber die Empfindungen in Schlundröhre und im Magen," Mitt. a. d. Grenzgeh. Med. u. Chir., $\mathrm{XX}$ (1909), 445.

Zuincke, H. "Ueber Temperatur und Wärmeausgleich im Magen," Arch.f. exp. Path. u. Pharm., XXV (I889), 375. 


\section{INDEX}

Abdomen, effect of massage of, on hunger, 200.

Acidity of human gastric juice, 254 .

Acids, in stomach, influence of, on hunger, I 7 I, I 75 .

Age, variations in hunger with, II9.

Akoria, 266.

Alcohol: influence of, on appetite, 180; on hunger, 178 .

Alcoholic gastritis, hunger and appetite in, $27 \mathrm{I}$.

Alkalies in stomach, influence of, on hunger, 171 , 265.

Allotriophagia, 267.

Ammino acids, in gastric juice, 253.

Ammonia, in gastric juice, $25 \mathrm{I}$.

Animal behavior, influence on, of hunger, $8, \mathrm{I}_{34}, \mathrm{I} 36, \mathrm{I} 44, \mathrm{I} 47$.

Anorexia, 266, 286.

Appetite: gastric factors in, $99, \mathrm{II}_{5}$; inheritance, factors in, $I I$; in disease, $26 \mathrm{r}$; in fevers, 278,286 ; in prolonged starvation, 134; nature of, 98 ; perverted, 267 ; relation of, to hunger, Io, 98 ; relation of, to thirst, 12 ; theories of, 96 .

Appetite gastric juice: acidity of, 254; chemistry of, 248; importance of, in normal digestion, 247; secretion of, $235,240$.

Bile, in stomach, 132 .

Bitter tonics: influence of, on hunger, 290; on food consumption, 300; on gastric juice secretion, 293; nature of action of, 289,302 .

Boldyreff, work of, on stomach in hunger, 27,33 .

Borborygmi, in hunger, 26,82 .

Boring, work of, on hunger, 25, 105.

Brain, hunger centers in, $20,149,215,216$.

Bulimia: in duodenal fistula, 24 ; in resection of stomach, 23; in various diseases, $26 \mathrm{I}$.

Busch, work of, on hunger, 24.
Cachexia, action of bitter tonics in, 299, 300.

Cannon, work of, on hunger, 19, 28, 35 .

Carbon dioxide in stomach, influence of, on hunger, 180 .

Cardia, in hunger, 77.

Chemotropism, in appetite, I I, 99.

Cerebrum: influence of, on hunger contractions, 149; hunger centers in, 216.

Cold, effect of, on hunger, 7, 9, 206, 2 I I.

Continuous secretion of gastric juice, 233 , 234.

Crop, hunger contractions of, in birds, $5 \mathrm{I}$.

Diabetes mellitus, hunger in, 279.

Diabetic blood, influence of, on hunger, 220.

Disease, hunger and appetite in, 26r.

Distemper, hunger in, 273.

Drugs, influence of, on hunger, 229.

Emotions, influence of, on hunger, I 5 I, I82.

Epigastric "emptiness," feeling of, in hunger, 93.

Epinephrin, influence of, on hunger, 229.

Esophagus: in hunger, $7,7 \mathrm{I}, 8 \mathrm{I}$; sensibility of, 104 .

Fevers, hunger and appetite in, 278, 286.

Gall-bladder infections, gastric pain in, 283.

Gastric cancer, hunger in, 280.

Gastric hunger contractions: after section of stomach nerves, I4I ; chemical control of, 2I7; effect of hemorrhage on, 222; in dogs, 138 ; in infants, 40 , I 19 ; in man, 36 , 126 ; in rabbits, 143 ; in reptilia, 53 ;

-conditions of disease: alcoholic gastritis, 27I; diabetes mellitus, 277; distemper, 273; fevers, 276 , 286; gallbladder infections, 283 ; gastric cancer, 280 ; gastric ulcer, 281 ; gastritis, 278 ; 
mange, 268; pancreatic diabetes, 268; parothyroid tetany, 273; peritonitis, 273; pneumonia, 273; pylorospasm, 284; pyloric stenosis, 275 ; tonsillitis, 278 ;

-in the isolated stomach, 160 ;

-influence of, on circulation, 88 ; on reflexes, 85 ; on salivation, 89 ;

-influence on, by chemicals in the mouth, I6 I, I66; chemicals in the stomach, I7I; decerebration, I49; diabetic blood, 22I; starvation blood, 219; section of stomach nerves, 158 ;

-mechanism of, 169,183 ; relation of, to digestion contractions, 56 ; to feeding habits, I 53, I 85 .

Gastric juice: auto-digestion of, 235 ; chemistry of, 248 ; quantity of, 243 , 245 ; secretion of, 233,240 .

Gastric mucosa: pain sensation from, IO2; tactile sensation from, IO3; temperature sensation from, I04; sensation of nausea from, Ir 3 .

Gastric tonus: relation of, to hunger, 67 ; in prolonged starvation, $128, \mathrm{r}_{4} \mathrm{I}$.

Gastric ulcer, hunger in, 264.

Gastritis, hunger in, 278.

Haller, theory of, hunger, 17 .

Headache, in hunger, 92, 94, I34.

Hemorrhage, influence of, on hunger, 222.

Herbivora, hunger in, 4I, 48, 50 .

Hertz, work of, on sensibility of stomach, I03, I05, III, 264.

Hunger: cause of, $6 \mathrm{r}$; general phenomena of, 7 ; influence of, on behavior, 8 , $\mathrm{r}_{34}, \mathrm{I} 36, \mathrm{r} 44, \mathrm{r} 47$; in disease conditions, 261 ; in higher animals, 6, 30, 125; in plants, 5 ; in protozoa, 1 ; method of objective study of, $3 \mathrm{I}$; relation of, to appetite, 96 ; relation of, to the need of food, 22, I53, I85; theories of, 16 .

Hunger centers: in cerebrum, 216; in medulla, 214; in optic thalami, $2 \mathrm{I}_{5}$.

Hunger pains: in gastric ulcer, 263, 28I.

Hunger tetanus of stomach: in dogs, 44 ; in man, $37,65,212$; in pancreatic diabetes, 268; in prolonged starvation, 125 ; in pyloric stenosis, 275 .

Hydrochloric acid, rôle of, in hunger pains, 264.

Hyperacidity, in stomach, 256.
Infants: feeding of, I 21 ; hunger of, 40 , II9.

Inheritance: of chemotropic elements of appetite, Ir, 99; of feeding reflexes, 9 , I2.

Inhibition of hunger: by bitter tonics, 292; by cold stimulation of the skin, 206, 211 ; by disease, $26 \mathrm{I}$; by the emotions, 151 , 182 ; by heat stimulation of the skin, 206; by mastication, I65; by running, 2ro; by sapid substances in the mouth, 160, 167, 189, 193; by smoking, 199; by stimulation of gastric mucosa, 169; by stimulation of intestinal mucosa, 194; by swallowing, I67.

Instincts, relation of, to hunger and appetite, 9 .

Intestines, contractions of, in hunger, $8 \mathrm{r}$.

Luckhardt, work of, on hunger, 220, 222 , 230, 268, 27 I, 277,280 .

Mange, increased hunger in, 268.

Mastication, inhibition of hunger by, 165 .

Medulla, hunger centers in, 2 ro.

Memory, in appetite, 97.

Moorhead, work of, on bitters, 299, 300.

Nausea: in prolonged starvation, $\mathrm{I} 35$; relation of, to hunger, $7,92,94$, I 3 .

Nicolai, work of, on hunger, 25 .

Optic thalami, rôle of, in hunger, $\mathrm{r}_{54}, 2 \mathrm{r}_{5}$.

Pancreatic diabetes, hunger in, 268.

Parathyroid tetany, hunger in, 273.

Paroexia, 267.

Patterson, work of, on hunger, 52, 120 , I23.

Pepsin, concentration of, in gastric juice, 259.

Peritonitis, hunger in, 273.

Phagocytosis, in protozoa, influence of hunger on, 3,4 .

Phlorhizin glucosuria, 230.

Physical exercise, effect of, on hunger, 203, 205, 210.

Pituitrin, influence of, on hunger, 229.

Plants, hunger in, 5 .

Pncumonia, hunger and appetite in, 273. 
Polyphagia: in man, 266; in mange, 269; in pancreatic diabetes, 268 .

Pressure on abdomen, effect of, on hunger, 200.

Protozoa, hunger behavior of, 2,4 .

Pyloric stenosis, influence of, on hunger, 275.

Pylorospasm, hunger contractions in, 284 .

Pylorus, effect of patency of, on hunger, 23.

Rectal feeding, influence of, on hunger, 25.

Rogers, work of, on hunger, $51,56,143$, I 5 I, 154, 194, 2 I 5 .

Rumen, hunger contractions of, $5 \circ$.

Ruminants, hunger in, 50.

Salivation, in hunger, 89 .

Salt hunger, 14 .

Satiety, origin of sensation of, II I.

Sleep, hunger contractions in, 150 .

Smoking, influence of, on hunger, 199.

Splanchnic nerves, effect of section of, on hunger, 155 .

Starvation: after-effects of, $\mathrm{r} 37$; discomforts of, 138 ; gastric hunger contractions in, 126 ; gastric juice in, 128 ; gastric tonus in, 26, 14I; hunger and appetite in, 125, 134; literature on, r44.
Starvation blood, influence of, on hunger, 219.

Stomach: action of bitter tonics on, 289; digestion contractions of, 55; extirpation of, in relation to hunger, 23 ; fluid content of empty stomach, 232; hunger contractions of, in amphibia and reptilia, 53; in birds, $5 \mathrm{I}$; in dogs, $4 \mathrm{I}$; in herbivora, 48; in infants, 40; in man, 36; sensibility of, to appetite, 99 , II 5 ; to pain, 102; to tempcrature, ro4; to touch, 103 .

Stomach pouch (Pavlov), hunger contractions of, 225.

Stomach pulse, 39 .

Taste sense: influence of, on hunger, 163 ; importance of, in appetite secretion, $235,240,247$; rôle of, in appetite, 98 .

Thirst, lack of appetite elements in, 13.

Tonsillitis, hunger and appetite in, 278 .

Vagi centers, in medulla: reflex control of, $169,210,218$; tonus of, 206, 214.

Vagi nerves: effect of section of, on hunger contractions, 156 ; relation to hunger sense, 22, 214; rôle of, in gastric juice secretion, 234 .

Water in stomach, influence of, on hunger, I 7 I.

Weakness, feeling of, in hunger, 7, 92, $135,137$. 




\section{THE LIBRARY}

UNIVERSITY OF CALIFORNIA

San Francisco Medical Center

THIS BOOK IS DUE ON THE LAST DATE STAMPED BELOW

Books not returned on time are subject to fines according to the Library Lending Code.

Books not in demand may be renewed if application is made before expiration of loan period.

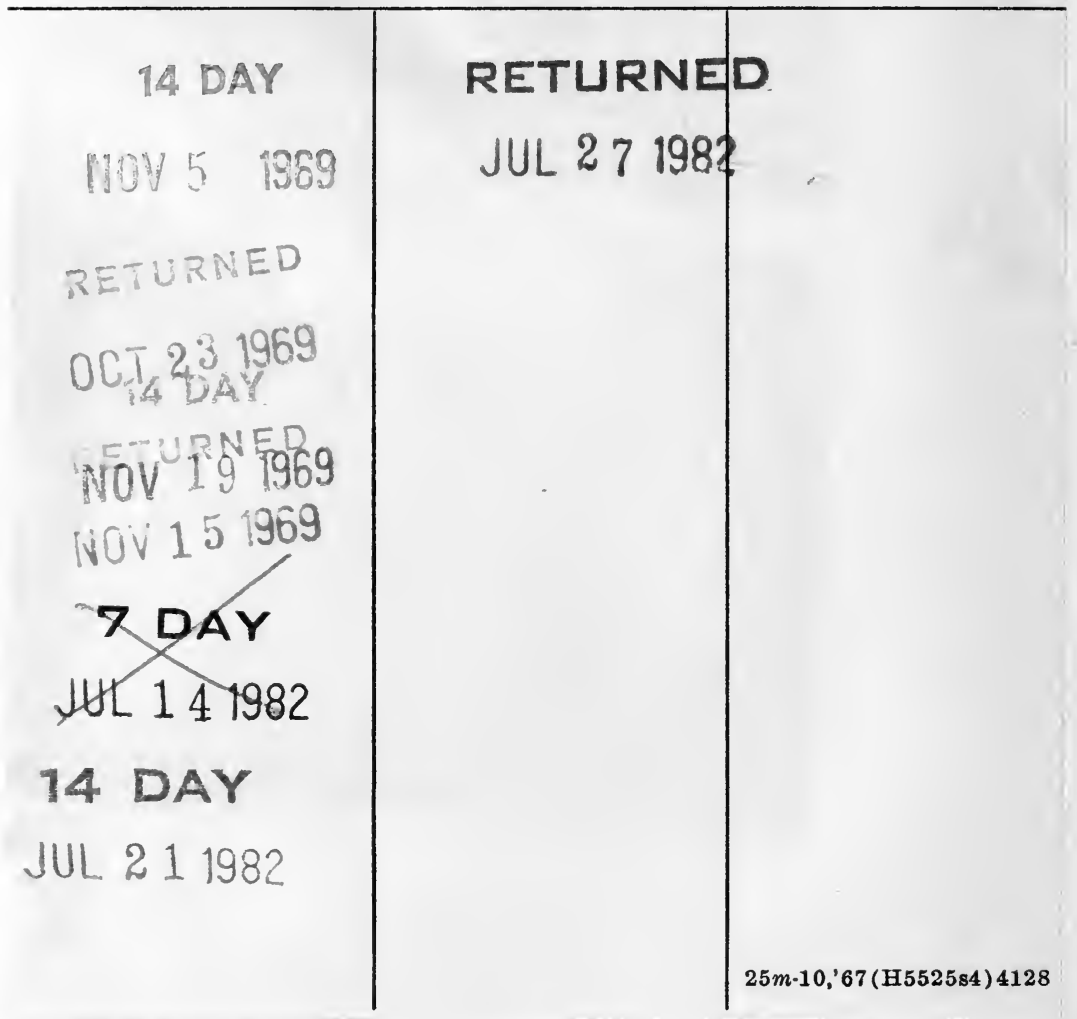




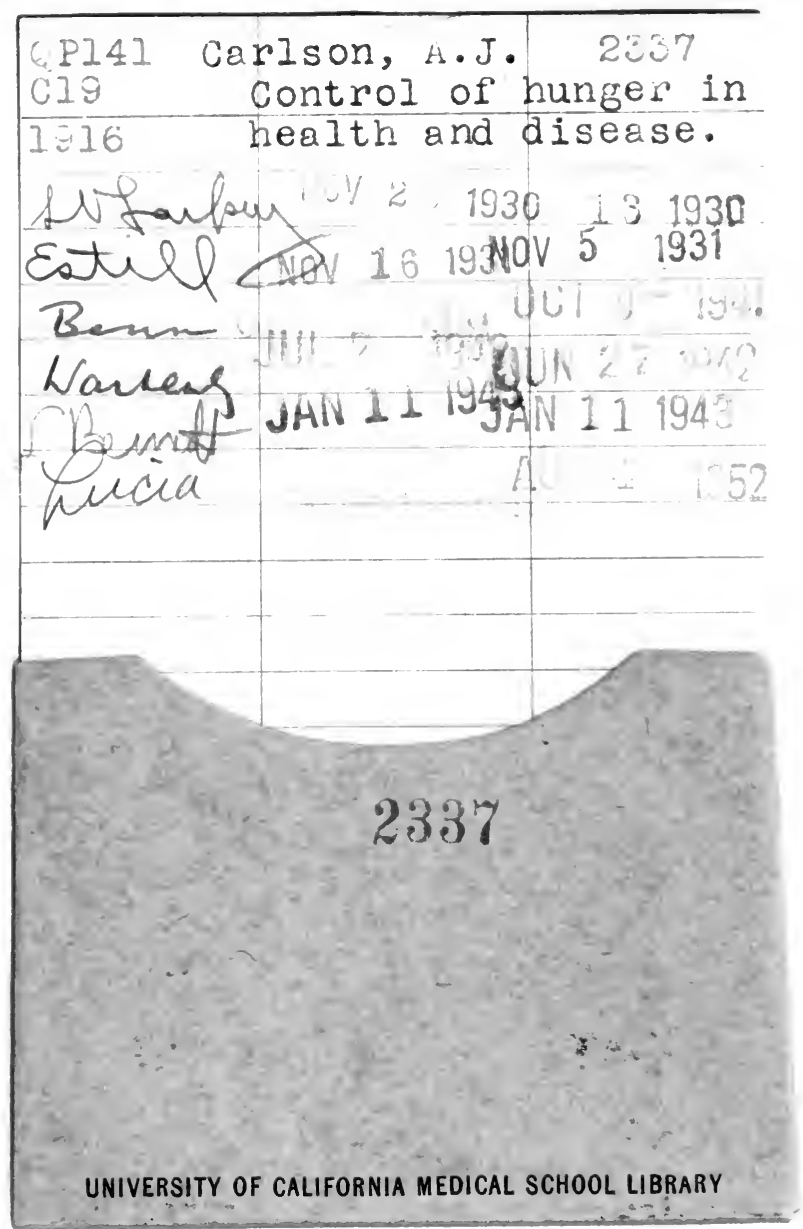


